\title{
Meat consumption and cancer risk : an epidemiological analysis from multiple perspectives
}

Citation for published version (APA):

Gilsing, A. M. J. (2014). Meat consumption and cancer risk : an epidemiological analysis from multiple perspectives. [Doctoral Thesis, Maastricht University]. Maastricht University. https://doi.org/10.26481/dis.20141218ag

Document status and date:

Published: 01/01/2014

DOI:

10.26481/dis.20141218ag

Document Version:

Publisher's PDF, also known as Version of record

\section{Please check the document version of this publication:}

- A submitted manuscript is the version of the article upon submission and before peer-review. There can be important differences between the submitted version and the official published version of record.

People interested in the research are advised to contact the author for the final version of the publication, or visit the DOI to the publisher's website.

- The final author version and the galley proof are versions of the publication after peer review.

- The final published version features the final layout of the paper including the volume, issue and page numbers.

Link to publication

\footnotetext{
General rights rights.

- You may freely distribute the URL identifying the publication in the public portal. please follow below link for the End User Agreement:

www.umlib.nl/taverne-license

Take down policy

If you believe that this document breaches copyright please contact us at:

repository@maastrichtuniversity.nl

providing details and we will investigate your claim.
}

Copyright and moral rights for the publications made accessible in the public portal are retained by the authors and/or other copyright owners and it is a condition of accessing publications that users recognise and abide by the legal requirements associated with these

- Users may download and print one copy of any publication from the public portal for the purpose of private study or research.

- You may not further distribute the material or use it for any profit-making activity or commercial gain

If the publication is distributed under the terms of Article $25 \mathrm{fa}$ of the Dutch Copyright Act, indicated by the "Taverne" license above, 

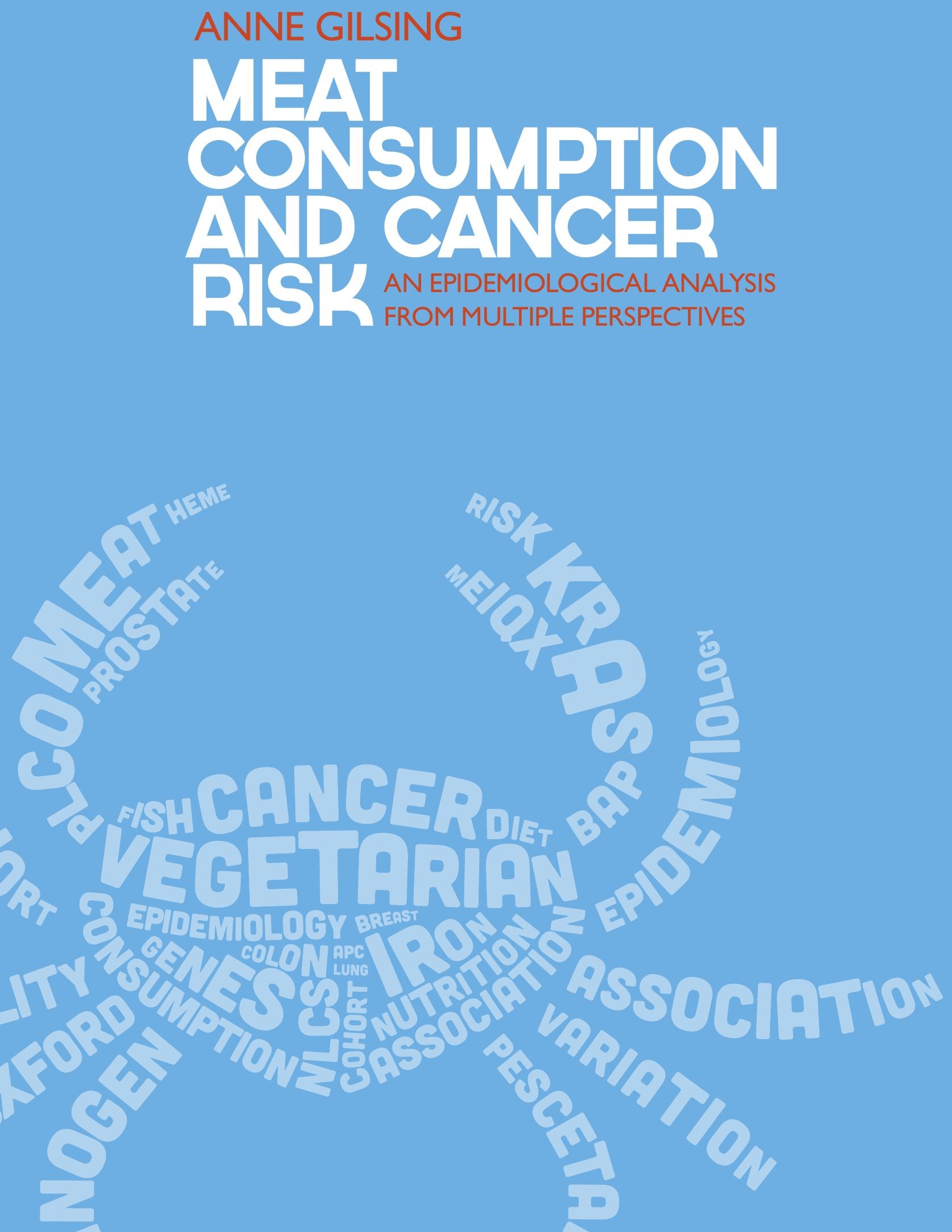
The work presented in this thesis was performed within GROW, School for Oncology and Developmental Biology at Maastricht University. This PhD research was supported by Wereld Kanker Onderzoek Fonds (WCRF-NL) and the Dutch Cancer Society (KWF).

Meat consumption and cancer risk - an epidemiological analysis from multiple perspectives

ISBN

9789461593764

Cover Design: Bram Giesen

Lay-out: Anne Gilsing

Printed by: Datawyse - Universitaire Pers Maastricht

Copyright (C) Maastricht 2014, Anne Gilsing

All rights reserved. No part of this thesis may be reproduced or transmitted in any form or by any means, electronic, or mechanical, including photocopying, recording or any information storage or retrieval system, without permission from the author in writing, or when appropriate, from the publishers of the publications. 


\title{
Meat consumption and cancer risk
}

\section{An epidemiological analysis from multiple perspectives}

\author{
PROEFSCHRIFT \\ ter verkrijging van de graad van doctor \\ aan de Universiteit Maastricht, \\ op gezag van de Rector Magnificus, Prof. dr. L.L.G. Soete \\ volgens het besluit van het College van Decanen, \\ in het openbaar te verdediging \\ op donderdag 18 december 2014 om 14:00 uur \\ door \\ Antonetta Martina Jacoba Gilsing
}

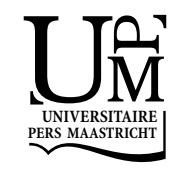




\section{Promotor}

Prof. dr. P.A. van den Brandt

Prof. dr. M.P. Weijenberg

Copromotor

Dr. L.J. Schouten

\section{Assessment committee}

Prof. dr. F.C. Ramaekers (chairman)

Dr. R.W. Godschalk

Prof. dr. E. Kampman (Wageningen University and Research Centre)

Prof. dr. A.A.M. Masclee

Prof. dr. M.P. Zeegers

\section{Wereld Kanker

Financial support for the printing of this thesis was kindly provided by Wereld Kanker Onderzoek Fonds (WCRF-NL). 
Voor ons pap en ons mam 



\section{CONTENTS}

Chapter 1 General Introduction

A dietary pattern approach

Chapter 2 Meat consumption and changes in BMI over time: longitudinal

analyses from the Netherlands Cohort Study

Chapter 3 Serum concentrations of vitamin B12 and folate in British male omnivores, vegetarians and vegans: results from a crosssectional analysis of the EPIC-Oxford cohort study

Chapter 4 The Netherlands Cohort Study - Meat Investigation Cohort;

a population-based cohort oversampled with vegetarians, pescetarians and low meat consumers

Chapter 5 Colorectal cancer risk in vegetarians and low meat consumers:

a prospective investigation

Chapter 6 Vegetarianism, low meat consumption and the risk of lung, prostate and female breast cancer in a population based cohort study

\section{A mechanistic approach}

Chapter 7 Consumption of dietary fat and meat and the risk of ovarian cancer in the Netherlands Cohort Study

Chapter 8 Meat-related mutagen exposure, xenobiotic metabolizing gene polymorphisms and the risk of advanced colorectal adenoma and cancer

Chapter 9 Dietary heme is associated with colorectal cancer with

$\mathrm{G}>\mathrm{A}$ mutations in KRAS and APC genes

Chapter 10 General discussion

Summary/Samenvatting

Valorisation

Etcetera

(dankwoord, about the author, list of publications) 



\section{CHAPTER 1}

General introduction 
The quest to unravel the association between meat and cancer risk started in 1975, when Armstrong and Doll observed that international variation in meat consumption was strongly correlated with cancer incidence and mortality, particularly cancer of the colon [1]. In addition, rapid changes in cancer rates were observed among populations migrating from low to high meat consumption countries [2]. This early ecological evidence generated a great deal of scientific interest to further examine the meat-cancer association using individual-level study designs. Since then, hundreds of epidemiological studies from many countries with diverse diets have reported on the relation between meat and cancer risk. However, despite these efforts, the effect of meat consumption on cancer risk remains a controversial issue.

In order to further elucidate the role of meat consumption in the etiology of cancer it is important to examine the total range of meat consumption, including extreme intake levels. In addition, more insight on the association between meat consumption and cancer risk can be obtained when epidemiological analyses are conducted at the level of meatrelated dietary patterns, total meat intake, subtypes of meat, and individual meat carcinogens. By including molecular markers in epidemiologic studies, it is possible to shed more light on the biological pathways linking meat consumption to cancer risk. When the results from such multiple lines of research are consistent, evidence on the association between meat and cancer risk is enhanced.

For the abovementioned reasons, this thesis set out to investigate the association between meat consumption and cancer risk using a multi-dimensional epidemiological approach. The first chapters of this thesis focus on meat-related dietary patterns in relation to cancer risk (i.e. vegetarian and low meat diets), whereas specific mechanisms of meat-related carcinogenesis (i.e. dietary fat, heterocyclic amines (HCAs), polycyclic aromatic hydrocarbons (HCAs), nitrite/nitrate, and heme iron) are examined in the second part of this thesis.

\section{MEAT CONSUMPTION AND CANCER: THE EPIDEMIOLOGICAL EVIDENCE}

This section briefly summarizes the existing epidemiological evidence regarding the role of meat consumption in the etiology of cancer.

Research indicates that the cancer promoting effect of meat differs both by meat subtype and cancer endpoint. The cancer site most consistently associated with meat intake is the colorectum. In 2007, a review and meta-analysis commissioned by the World Cancer Research Fund / American Institute for Cancer Research concluded that there was convincing evidence for a positive association between red meat and processed meat intake and colorectal cancer [3]. Based on their conclusions, the WCRF recommends people to consume less than $\mathbf{5 0 0}$ grams of red meat per week and to avoid the consumption of processed meat [3]. Although the conclusions of the WCRF report have been questioned by some $[4,5]$, the most recent meta-analysis including data from 26 prospective studies supports limiting the amount of red and processed meat consumption for colorectal cancer prevention [6]. Chan et al indicated that for every $50 \mathrm{~g} /$ day increase of fresh red meat or processed meat the risk of colorectal cancer increased by $17 \%$ and $18 \%$, respectively [6]. 
In contrast, evidence for an association between red and processed meat and other major cancers such as prostate [7], breast [8], lung [9] and pancreatic cancer [10] is less abundant and less consistent across studies. Some rarer type cancers like renal cell, endometrial, ovarian, esophageal, and stomach cancer have been hypothesized to be associated with red and processed meat intake, but confirmative evidence from prospective population based data are sparse [11-15]. However, definitions and consumption patterns of red and processed meat differ between populations and across studies. Conclusions and recommendations are therefore not as clear as they could be [3].

In contrast to red and processed meat, white meat (i.e. poultry) has not been as extensively investigated in prospective epidemiologic studies [3]. Nonetheless, the available studies generally reported null or cancer preventive effects (e.g. ref 16-19). Moreover, although the nutrient composition of meat is strongly determined by a number of factors including the animal of origin, only a few studies examined cancer risk in relation to meat subtypes (e.g. beef, pork, lamb, or offal (i.e. organ meats)).

\section{A DIETARY PATTERN APPROACH}

Individuals who consume a diet void of all meat provide an interesting opportunity to further elucidate the relation between meat consumption and cancer risk. Studies of vegetarians can nicely complement the above mentioned epidemiological studies that are conducted at the food group level (i.e. meat (subtypes)). Although vegetarian diets are defined by the absence of all meats, fish and shellfish, several subtypes can be defined. For example, lacto-ovo vegetarians include both eggs and dairy products in their diet, lactovegetarians consume dairy products but no eggs, and ovo-vegetarians consume eggs but no dairy products. In addition, pescetarians abstain from eating all meat with the exception of fish whereas vegans exclude all animal-derived products and ingredients from their diet. Thus, the choice of a specific vegetarian diet may determine the effect on the nutritional status and cancer risk. While the worldwide demand for meat is expected to grow considerably over the next decades, the total number of vegetarians is also rapidly increasing as a result of nutritional, ethical, and more recently, environmental concerns [20]. This transition is accompanied by an increase in the number of meat reducers [21]. However, while the health effects of vegetarian diets are receiving increasing scientific interest, little to no research has been conducted in the area of low meat consumers (in this thesis defined as individuals who consume meat for 1 day/week).

\section{Vegetarian and low meat diets}

A few prospective cohort studies specifically set out to study cancer incidence and mortality in vegetarians. A pooled estimate of mortality rates in five prospective cohort studies (including the Adventist Health Study I (AHS))[22], the Adventists Mortality Study [23], the Health Food Shoppers Study [24], the Heidelberg Study [25], and the Oxford Vegetarian Study [26]) reported no large differences in (total) cancer mortality between vegetarians and non-vegetarians [27]. More recent results from the AHS II also revealed no significant associations between vegetarianism and cancer mortality [28]. Fewer and more inconsistent data are available on the role of vegetarian diets in cancer incidence. In the United States, results from the AHS I suggest that vegetarians are at a reduced risk of developing colon cancer and prostate cancer compared to meat eaters [29]. Preliminary 
analyses from AHS II report lower risks of cancer of the gastro-intestinal tract and respiratory tract among vegetarians, and suggest that vegans had lower risks of overall cancer and female specific cancers [30]. In the United Kingdom, the Oxford Vegetarian Study [31] revealed no large differences in colorectal cancer incidence between vegetarians and non-vegetarians, whereas results from the European Prospective Investigation into Cancer and Nutrition (EPIC)-Oxford cohort indicate that vegetarians were at an increased risk [32]. The latter strongly contrasts the finding that red and processed meat are convincing causes of colorectal cancer [6]. In addition, compared to non-vegetarians, vegetarians were at lower risk of stomach cancer, bladder cancer, and cancers of the lymphatic and hematopoietic tissues when data from EPIC-Oxford and the Oxford Vegetarian Study were pooled [33]. The UK Women's Cohort study suggests that women who do not eat meat have a lower risk of breast cancer [34] than high meat consumers, but studies that compared vegetarians to non-vegetarians (including the UK Women Cohort study) reported no associations [29, 32, 33, 35]. Overall, too few data from large cohort studies are present to formulate more than general conclusions concerning the role of vegetarianism in the development of cancer [3].

Some of the inconsistencies in previous findings may be due to differences in sampling strategies between studies; some studies stem from convenience samples that have likely also recruited more health-conscious non-vegetarians. Other inconsistencies may have resulted from differences in definition and operationalization of vegetarianism; only a few studies identified vegetarians based on extensive dietary data collection techniques [22, 36], whereas others reported to have classified vegetarians by means of several broader questions relating to the overall consumption of animal products [25, 37, 38], or to have used self-reported vegetarianism [24] as inclusion criterion. However, there is substantial inconsistency in how people self-identify $[39,40]$, and the usefulness and reliability of selfdefined vegetarianism in etiological studies of cancer remains unclear.

Appropriately planned vegetarian diets have shown to be consistent with the current dietary guidelines in all stages of the lifecycle [41]. Nonetheless, meat provides an important source of fat, protein, essential amino acids, and vitamins and minerals such as iron, zinc, and vitamin B12. Studies suggest that vegetarians have lower circulating concentrations of vitamin B12 compared to meat eaters depending on the level of animal product restriction in the diet [42]. Vegetarians have also inconsistently been reported to have lower serum levels of iron, vitamin D, omega-3 fatty acids, and zinc (summarized in ref 43).

Low meat diets may be less prone to such dietary deficiencies, and may consequently result in a similar or perhaps even lower cancer risk than vegetarian diets. However, within the existing studies of meat, vegetarianism and cancer risk, little to no attention has been paid to the lower end of the meat consumption spectrum. This may partly result from the relatively narrow intake distribution of meat intake in Western countries. In the Netherlands, less than $20 \%$ of the population consumes meat for $\leq 2$ days per week for the hot meal [21]. This constrains our capacity to examine the cancer risk associated with (very) low intake levels. Studying the meat cancer association in population based studies enriched with vegetarians and low meat consumers increases the heterogeneity of meat exposure which may increase the power to detect an effect. 


\section{Challenges associated with the dietary pattern approach}

Although vegetarian and low meat diets are only defined by the level of meat and/or fish consumption, they are likely to be interrelated with other dietary and lifestyle factors [38] that may have an independent effect on cancer occurrence. For example, vegetarians have higher levels of fruit and vegetable consumption, physical activity, and a lower prevalence of smoking and alcohol use [38]. In addition, a diet high in meat consumption may increase the risk of developing obesity [44, 45], which is an independent risk factor of many cancers. However, whether the health effects of no and low meat diets can be explained by factors other than meat consumption remains controversial. Most studies examining cancer incidence in vegetarians only adjusted for confounding variables outside the diet [29, 30, 32,33 ] and little is known about the potential explanatory role of these multiple connected factors.

The vegetarian and low meat dietary patterns are likely to vary by sex, socioeconomic status, religion, ethnic group and culture. In addition, no and low meat consumers are likely to be health conscious. Because it has been postulated that these self-selection factors may confound any association with health outcomes [46], it is essential to replicate results in diverse populations. When similar cancer risk estimates are observed in different populations of vegetarians and low meat consumers, it is more likely that the results can be attributed to the diet as such rather than underlying selection processes because these will also differ between populations.

An association with an overall dietary pattern does not enhance biological insight or mechanistic understanding. Studying dietary patterns should therefore be considered as a complementary approach to understand the relation between meat consumption and cancer risk that preferably would be combined with analysis at the levels of meat (subgroups) and meat carcinogens.

\section{A MECHANISTIC APPROACH}

The second part of this thesis focuses on mechanisms of meat-related carcinogenesis in an attempt to further elucidate the role of meat consumption in the etiology of cancer. If the primary cancer causing mechanism(s) of meat consumption can be established, it may be possible to change or intervene on the production processes that lead to carcinogen formation. In this section an overview of the most widely accepted and studied hypotheses is provided, but this list is not exhaustive.

\section{Dietary fat}

Dietary fat, particularly saturated fat, has long been suspected to be responsible for the meat-cancer association. Dietary fat increases bile acid secretion in the gut which acts as aggressive surfactants that may promote colorectal carcinogenesis [47]. Additionally, high intake of dietary fat is suggested to alter endogenous hormone levels, thereby possibly increasing the risk of hormone-related cancers such as breast, ovarian, endometrial, and prostate cancer. However, epidemiological studies have been inconsistent $[3,48]$ and intervention studies aimed at lowering total fat intake failed to confirm any association with cancer risk (e.g. ref 49-51) except for cancer of the ovaries [50]. 


\section{Heterocyclic amines and polycyclic aromatic hydrocarbons}

More recent evidence suggests a role for meat-related specific mutagens. These include the formation of heterocyclic amines (HCAs) during high temperature cooking of meat when creatine or creatinine, amino acids, and sugar react to form a variety of HCAs [52]. This reaction is a function of both cooking temperature and cooking time. Over 20 HCA compounds have been identified, but the most abundant HCAs in cooked meat are 2amino-3,8-dimethylimidazo[4,5-f]quinoxaline (MelQx), 2-amino-3,4,8-trimethylimidazo[4,5-f]- quinoxaline (DiMelQx) and 2-amino-1-methyl-6-phenyl-imidazo[4,5-b]pyridine (PhIP). Polycyclic aromatic hydrocarbons (PAHs) are a second class of meat-related mutagens that are predominantly found in grilled, barbequed, and smoked meat. Preparing meat over a direct flame results in fat and meat juices dripping onto the hot fire causing PAH containing smoke to coat the surface of the meat. While more than 100 different types of HCAs have been identified, extensive information concerning meat carcinogenesis is only available for benzo[a]pyrene (B[a]P), which is considered a marker of total PAH exposure. Although there is ample evidence from in vitro and animal studies that HCAs and PAHs can damage DNA and cause tumors in multiple organs [53-57], evidence in humans remains inconsistent [3]. Over the past 10 years, an increasing number of epidemiological studies have included surrogates of HCA and PAH exposure in their dietary assessment (i.e. meat cooking technique, doneness level and surface browning of the meat) [58]. This information can be linked to databases containing the HCA and PAH content of numerous meats prepared by various cooking methods and doneness levels, to estimate intake levels [58]. Previous studies have reported that high exposure to meat-related HCAs and PAHs may be associated with the risk of cancers including colorectal, breast, prostate, pancreas, lung, stomach, and esophagus. A review published in 2009 suggests that the strongest and most consistent associations are observed for PhIP intake (see ref 59 and references therein). Associations between MelQx, DiMelQx and B[a]P were less consistent across studies and cancer endpoints [59], possibly due to their lower exposure concentrations.

\section{Dietary heme and $\mathbf{N}$-nitroso compounds}

Dietary heme iron may also explain meat induced carcinogenesis. Heme is present at fivefold higher concentrations in red than white meat [60] and could explain why white meat, in contrast to red meat, is not associated with cancer risk. Most epidemiologic research has examined heme iron in relation to colorectal cancer and a recent meta-analysis of five prospective studies indeed showed a consistent, though modest, increased risk of $18 \%$ comparing the highest to the lowest category of heme iron intake. Much less is known about the effect of heme on other cancer endpoints but some studies are indicative of an increased risk of e.g. esophageal [14, 61], gastric cancer [61], endometrial cancer [62], and lung cancer [63]. The hypothesis that heme iron may be associated with an increased cancer risk is strengthened by the observation that heme iron catalyzes the endogenous formation of N-nitroso compounds (NOCs) in humans [64]. In addition, NOCs may also be formed in processed meats when nitrite is added during the curing process [65]. NOCs are some of the most powerful chemical carcinogens, inducing tumors in multiple organ sites in numerous animal species [53]. Both heme iron and NOCs may also catalyze the formation of reactive oxygen species that can cause DNA damage $[66,67]$ and heme is also thought to promote colonic cytotoxicity and increased cell proliferation [68-70]. Nonetheless, mechanistic evidence on the role of heme iron in carcinogenesis from population based observational studies is lacking. 


\section{Molecular epidemiology to study meat-related carcinogenic pathways}

The previously described epidemiological studies of meat, meat carcinogens and cancer risk generally made use of traditional epidemiologic modes of data collection (e.g. using questionnaire data). Although this traditional approach has made pivotal contributions to specifying the association between meat and cancer risk, it often does not illuminate the exact biological mechanisms underlying the observed associations [71]. To open this "blackbox", we need to move towards the field of molecular epidemiology and integrate biomarker data into our epidemiologic studies [72]. Biomarkers of exposure (e.g. circulation nutrient/carcinogen concentrations), susceptibility (e.g. genetic variation) and/or effect (e.g. molecular signatures in tumors) are currently being included in observational studies and may increase our understanding of the role of meat consumption in cancer risk.

For example, the cancer risk posed by HCAs, PAHs, and NOCs depends on the extent to which they are metabolized: they undergo a series of chemical reactions in the human body during which they can be activated or detoxified by phase I and phase II xenobiotic metabolizing enzymes (XMEs)[73, 74]. Single nucleotide polymorphisms (SNPs) in the genes encoding these XMEs may modify the ability to activate or detoxify carcinogens. Associations between meat consumption and cancer risk may therefore be limited to genetically susceptible individuals. Nonetheless, studies examining interactions between $\mathrm{XME}$ polymorphisms, meat consumption and the risk of various cancer endpoints have reported mixed results (e.g. refs 75-81) but often had a small number of cases available or examined only a small set of SNPs from a limited number of candidate genes. Because the balance of activating and detoxifying enzymes is thought to influence carcinogen metabolism [82], comprehensive studies including numerous markers across multiple genes involved in xenobiotic metabolism are essential for studying this complex association.

Next to the study of gene environment interactions, clarification of the association between meat carcinogens and the risk of cancer by molecular tumor subtype may also lead to a better understanding of the suggested pathways linking meat consumption to cancer. More specifically, such somatic (or acquired) genetic alterations in the tumor can be viewed as a signature of long term internal and external exposures and therefore also of meat-related carcinogens. Observational studies that are able to investigate these mechanisms using biomarker data in large populations are essential in providing further evidence for the meat cancer association.

\section{RATIONALE, AIM, AND OUTLINE OF THIS THESIS}

The overall aim of this thesis was to investigate the association between meat consumption and cancer risk in order to explain the heterogeneity observed in previous studies using a multi-dimensional epidemiological approach (see Figure 1).

In the first part of this thesis, vegetarian and low meat dietary patterns were studied in relation to cancer risk. This section starts with two studies on meat consumption patterns and non-cancer health outcomes, since these may, in turn, affect the association between vegetarian and low meat diets and cancer risk. Because obesity is likely associated with a high meat dietary pattern but also an independent risk factor of many cancers, we were 
first interested to examine whether meat consumption was associated with prospective weight gain (14 years) using the Netherlands Cohort Study on diet and cancer (NLCS) [83] (Chapter 2). Secondly, individuals following a vegetarian diet are likely at higher risk of developing nutrient deficiencies which is why we set out to study serum vitamin B12 status in vegetarian, vegan and meat eating men using data from the EPIC-Oxford cohort (Chapter $3)$.

Because low meat consumers (i.e. individuals who consume meat for 1 day/week) may be less prone to develop nutrient deficiencies, we hypothesized they might also have an additional beneficial cancer preventive effect over vegetarians when compared to highmeat consumers. We therefore established an analytical cohort specifically designed to study the health effects of vegetarian and low meat diets focusing on cancer incidence and mortality. This population was extracted from the total NLCS and referred to as the NLCSMeat Investigation Cohort (NLCS-MIC). The NLCS aimed to over-represent vegetarians and low meat consumers at recruitment by intentionally contacting them through health food shops and magazines. In addition, the NCLS-MIC contains a considerable number of pescetarians. The NLCS used an extensive FFQ to assess dietary intake. This enabled us to classify vegetarians based on self-reporting (1 question) as well as on the more accurate and extensive FFQ data. Consequently, we could not only study vegetarianism and low meat consumption in relation to major cancer outcomes (colorectal, lung, female breast and prostate) but were also able to investigate the reliability of self-defined vegetarianism in relation to cancer risk estimates. As a result of the sampling strategy, this population has a large contrast in meat intake which should also aid the further specification of associations between subtypes of meat and cancer risk. Chapter 4 describes the composition and baseline characteristics of the NLCS-MIC cohort. In Chapter 5 and 6 associations are described between vegetarianism, low meat consumption and the risk of colorectal and lung, female breast and prostate cancer, respectively, using data from NLCSMIC.

In the second part of this thesis, we set out to study each of the proposed mechanisms of meat-related carcinogenicity in relation to the cancer endpoints most likely to be affected. Chapter 7 describes the association between meat and fat intake and the risk of ovarian cancer in the NLCS. In Chapter 8, we describe the combined effects of HCAs, PAHs and nitrite/nitrate intake and genetic variants in 18 phase I and phase II xenobiotic metabolizing enzymes genes in relation to advanced colorectal adenoma and colorectal cancer within the Prostate, Lung, Colorectal, and Ovarian Cancer (PLCO) Screening Trial. In Chapter 9 we studied heme iron in relation to the risk of colorectal cancer by specific molecular characteristics (i.e. mutation status of KRAS, APC and overexpression of P53) in the NLCS.

Chapter 10 provides a discussion of the main findings in light of some important strengths and limitations, and recommendations for future research. 


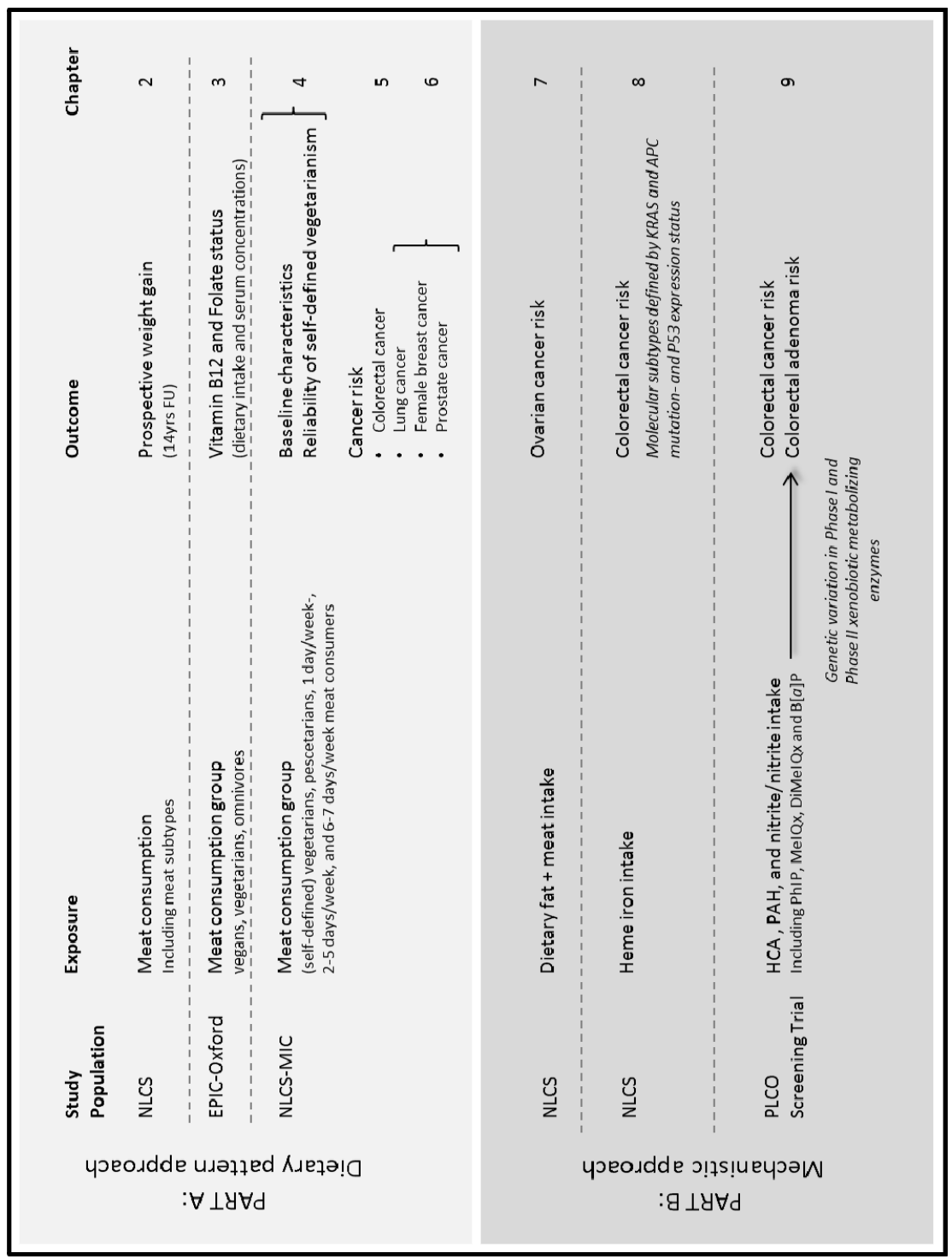

Figure 1: Schematic overview of the studies included in this thesis.

NLCS (Netherlands Cohort Study), EPIC (European Prospective Investigation into Cancer and Nutrition), NLCS-MIC (NLCS- Meat Investigation Cohort), PLCO Screening Trial (Prostate, Lung, Colorectal and Ovarian) Screening Trial. 


\section{REFERENCES}

1. Armstrong, B. and R. Doll, Environmental factors and cancer incidence and mortality in different countries, with special reference to dietary practices. Int J Cancer, 1975. 15(4): p. 617-31.

2. Dunn, J.E., Cancer epidemiology in populations of the United States-with emphasis on Hawaii and California-and Japan. Cancer Res, 1975. 35(11 Pt. 2): p. 3240-5.

3. World Cancer Research Fund/American Institute for Cancer Research, Food, Nutrition, Physical Activity, and the Prevention of Cancer: a Global Perspective. 2007, AICR: Washington DC.

4. Alexander, D.D. and C.A. Cushing, Red meat and colorectal cancer: a critical summary of prospective epidemiologic studies. Obes Rev, 2011. 12(5): p. e472-93.

5. Alexander, D.D., et al., Processed meat and colorectal cancer: a quantitative review of prospective epidemiologic studies. Eur J Cancer Prev, 2010. 19(5): p. 328-41.

6. Chan, D.S., et al., Red and processed meat and colorectal cancer incidence: meta-analysis of prospective studies. PLoS One, 2011. 6(6): p. e20456.

7. Alexander, D.D., et al., A review and meta-analysis of prospective studies of red and processed meat intake and prostate cancer. Nutr J, 2010. 9: p. 50.

8. Alexander, D.D., et al., A review and meta-analysis of red and processed meat consumption and breast cancer. Nutr Res Rev, 2010. 23(2): p. 349-65.

9. Yang, W.S., et al., Meat consumption and risk of lung cancer: evidence from observational studies. Ann Oncol, 2012. 23(12): p. 3163-70.

10. Larsson, S.C. and A. Wolk, Red and processed meat consumption and risk of pancreatic cancer: metaanalysis of prospective studies. Br J Cancer, 2012. 106(3): p. 603-7.

11. Daniel, C.R., et al., Large prospective investigation of meat intake, related mutagens, and risk of renal cell carcinoma. Am J Clin Nutr, 2012. 95(1): p. 155-62.

12. Arem, H., et al., A prospective investigation of fish, meat and cooking-related carcinogens with endometrial cancer incidence. Br J Cancer, 2013. 109(3): p. 756-60.

13. Salehi, M., et al., Meat, fish, and esophageal cancer risk: a systematic review and dose-response metaanalysis. Nutr Rev, 2013. 71(5): p. 257-67.

14. Keszei, A.P., et al., Red and processed meat consumption and the risk of esophageal and gastric cancer subtypes in The Netherlands Cohort Study. Ann Oncol, 2012. 23(9): p. 2319-26.

15. Kiani, F., et al., Dietary risk factors for ovarian cancer: the Adventist Health Study (United States). Cancer Causes Control, 2006. 17(2): p. 137-46.

16. Cross, A.J., et al., Meat consumption and risk of esophageal and gastric cancer in a large prospective study. Am J Gastroenterol, 2011. 106(3): p. 432-42.

17. Daniel, C.R., et al., Prospective investigation of poultry and fish intake in relation to cancer risk. Cancer Prev Res (Phila), 2011. 4(11): p. 1903-11.

18. Gonzalez, C.A., et al., Meat intake and risk of stomach and esophageal adenocarcinoma within the European Prospective Investigation Into Cancer and Nutrition (EPIC). J Natl Cancer Inst, 2006. 98(5): p. 345-54.

19. Linseisen, J., et al., Consumption of meat and fish and risk of lung cancer: results from the European Prospective Investigation into Cancer and Nutrition. Cancer Causes Control, 2011. 22(6): p. 909-18.

20. Fox, N. and K. Ward, Health, ethics and environment: a qualitative study of vegetarian motivations. Appetite, 2008. 50(2-3): p. 422-9.

21. Dagevos, H., et al., Vlees vooral(snog) vanzelfsprekend: consumenten over vlees eten en vleesminderen. LEU-rapport 2012-029, L.W. UR, Editor. 2012: Den Haag.

22. Beeson, W.L., et al., Chronic disease among Seventh-day Adventists, a low-risk group. Rationale, methodology, and description of the population. Cancer, 1989. 64(3): p. 570-81.

23. Snowdon, D.A., Animal product consumption and mortality because of all causes combined, coronary heart disease, stroke, diabetes, and cancer in Seventh-day Adventists. Am J Clin Nutr, 1988. 48(3 Suppl): p. 739-48.

24. Burr, M.L. and P.M. Sweetnam, Vegetarianism, dietary fiber, and mortality. Am J Clin Nutr, 1982. 36(5): p. 873-7.

25. Frentzel-Beyme, R., J. Claude, and U. Eilber, Mortality among German vegetarians: first results after five years of follow-up. Nutr Cancer, 1988. 11(2): p. 117-26. 
26. Thorogood, M., et al., Risk of death from cancer and ischaemic heart disease in meat and non-meat eaters. BMJ, 1994. 308(6945): p. 1667-70.

27. Key, T.J., et al., Mortality in vegetarians and nonvegetarians: detailed findings from a collaborative analysis of 5 prospective studies. Am J Clin Nutr, 1999. 70(3 Suppl): p. 516S-524S.

28. Orlich, M.J., et al., Vegetarian dietary patterns and mortality in Adventist Health Study 2. JAMA Intern Med, 2013. 173(13): p. 1230-8.

29. Fraser, G.E., Associations between diet and cancer, ischemic heart disease, and all-cause mortality in non-Hispanic white California Seventh-day Adventists. Am J Clin Nutr, 1999. 70(3 Suppl): p. 532S-538S.

30. Tantamango-Bartley, Y., et al., Vegetarian diets and the incidence of cancer in a low-risk population. Cancer Epidemiol Biomarkers Prev, 2013. 22(2): p. 286-94.

31. Sanjoaquin, M.A., et al., Nutrition, lifestyle and colorectal cancer incidence: a prospective investigation of 10998 vegetarians and non-vegetarians in the United Kingdom. Br J Cancer, 2004. 90(1): p. 118-21.

32. Key, T.J., et al., Cancer incidence in vegetarians: results from the European Prospective Investigation into Cancer and Nutrition (EPIC-Oxford). Am J Clin Nutr, 2009. 89(5): p. 1620S-1626S.

33. Key, T.J., et al., Cancer incidence in British vegetarians. Br J Cancer, 2009. 101(1): p. 192-7.

34. Taylor, E.F., et al., Meat consumption and risk of breast cancer in the UK Women's Cohort Study. Br J Cancer, 2007. 96(7): p. 1139-46.

35. Cade, J.E., et al., Common dietary patterns and risk of breast cancer: analysis from the United Kingdom Women's Cohort Study. Nutr Cancer, 2010. 62(3): p. 300-6.

36. Butler, T.L., et al., Cohort profile: The Adventist Health Study-2 (AHS-2). Int J Epidemiol, 2008. 37(2): p. 260-5.

37. Appleby, P.N., et al., The Oxford Vegetarian Study: an overview. Am J Clin Nutr, 1999. 70(3 Suppl): p. 525S-531S.

38. Davey, G.K., et al., EPIC-Oxford: lifestyle characteristics and nutrient intakes in a cohort of 33883 meat-eaters and 31546 non meat-eaters in the UK. Public Health Nutr, 2003. 6(3): p. 259-69.

39. Vinnari, M., et al., Identifying vegetarians and their food consumption according to self-identification and operationalized definition in Finland. Public Health Nutr, 2009. 12(4): p. 481-8.

40. Haddad, E.H. and J.S. Tanzman, What do vegetarians in the United States eat? Am J Clin Nutr, 2003. 78(3 Suppl): p. 626S-632S.

41. Craig, W.J., A.R. Mangels, and A. American Dietetic, Position of the American Dietetic Association: vegetarian diets. J Am Diet Assoc, 2009. 109(7): p. 1266-82.

42. Herrmann, W., et al., Vitamin B-12 status, particularly holotranscobalamin II and methylmalonic acid concentrations, and hyperhomocysteinemia in vegetarians. Am J Clin Nutr, 2003. 78(1): p. 131-6.

43. Craig, W.J., Nutrition concerns and health effects of vegetarian diets. Nutr Clin Pract, 2010. 25(6): p. 613-20.

44. Wang, Y. and M.A. Beydoun, Meat consumption is associated with obesity and central obesity among US adults. Int J Obes (Lond), 2009. 33(6): p. 621-8.

45. Appleby, P.N., et al., Low body mass index in non-meat eaters: the possible roles of animal fat, dietary fibre and alcohol. Int J Obes Relat Metab Disord, 1998. 22(5): p. 454-60.

46. Vandenbroucke, J.P., Observational research, randomised trials, and two views of medical science. PLoS Med, 2008. 5(3): p. e67.

47. Bruce, W.R., Recent hypotheses for the origin of colon cancer. Cancer Res, 1987. 47(16): p. 4237-42.

48. Kushi, L. and E. Giovannucci, Dietary fat and cancer. Am J Med, 2002. 113 Suppl 9B: p. 63S-70S.

49. Prentice, R.L., et al., Low-fat dietary pattern and risk of invasive breast cancer: the Women's Health Initiative Randomized Controlled Dietary Modification Trial. JAMA, 2006. 295(6): p. 629-42.

50. Prentice, R.L., et al., Low-fat dietary pattern and cancer incidence in the Women's Health Initiative Dietary Modification Randomized Controlled Trial. J Natl Cancer Inst, 2007. 99(20): p. 1534-43.

51. Martin, L.J., et al., A randomized trial of dietary intervention for breast cancer prevention. Cancer Res, 2011. 71(1): p. 123-33.

52. Jagerstad, M., et al., Formation of heterocyclic amines using model systems. Mutat Res, 1991. 259(34): p. 219-33.

53. Bogovski, P. and S. Bogovski, Animal Species in which N-nitroso compounds induce cancer. Int J Cancer, 1981. 27(4): p. 471-4. 
54. Ito, N., et al., A new colon and mammary carcinogen in cooked food, 2-amino-1-methyl-6phenylimidazo[4,5-b]pyridine (PhIP). Carcinogenesis, 1991. 12(8): p. 1503-6.

55. Ochiai, M., et al., Induction of intestinal tumors and lymphomas in C57BL/6N mice by a food-borne carcinogen, 2-amino-1-methyl-6-phenylimidazo[4,5-b]pyridine. Jpn J Cancer Res, 2002. 93(5): p. 47883.

56. Ohgaki, H., S. Takayama, and T. Sugimura, Carcinogenicities of heterocyclic amines in cooked food. Mutat Res, 1991. 259(3-4): p. 399-410.

57. Sugimura, T., Overview of carcinogenic heterocyclic amines. Mutat Res, 1997. 376(1-2): p. 211-9.

58. Sinha, R., et al., Development of a food frequency questionnaire module and databases for compounds in cooked and processed meats. Mol Nutr Food Res, 2005. 49(7): p. 648-55.

59. Zheng, W. and S.A. Lee, Well-done meat intake, heterocyclic amine exposure, and cancer risk. Nutr Cancer, 2009. 61(4): p. 437-46.

60. Service., U.S.D.o.A.a.A.R., USDA National Nutrient Database for Standard Reference, Release 21. Internet: Nutrient Data Laboratory Home Page, http://www.ars.usda.gov/nutrientdata (accessed 22 November 2011).

61. Ward, M.H., et al., Heme iron from meat and risk of adenocarcinoma of the esophagus and stomach. Eur J Cancer Prev, 2012. 21(2): p. 134-8.

62. Genkinger, J.M., et al., Long-term dietary heme iron and red meat intake in relation to endometrial cancer risk. Am J Clin Nutr, 2012. 96(4): p. 848-54.

63. Tasevska, N., et al., A prospective study of meat, cooking methods, meat mutagens, heme iron, and lung cancer risks. Am J Clin Nutr, 2009. 89(6): p. 1884-94.

64. Cross, A.J., J.R. Pollock, and S.A. Bingham, Haem, not protein or inorganic iron, is responsible for endogenous intestinal N-nitrosation arising from red meat. Cancer Res, 2003. 63: p. 2358-2360.

65. Santarelli, R.L., F. Pierre, and D.E. Corpet, Processed meat and colorectal cancer: a review of epidemiologic and experimental evidence. Nutr Cancer, 2008. 60(2): p. 131-44.

66. Hebels, D.G., et al., Radical mechanisms in nitrosamine- and nitrosamide-induced whole-genome gene expression modulations in Caco-2 cells. Toxicol Sci, 2010. 116(1): p. 194-205.

67. Tappel, A., Heme of consumed red meat can act as a catalyst of oxidative damage and could initiate colon, breast and prostate cancers, heart disease and other diseases. Med Hypotheses, 2007. 68(3): p. 562-4.

68. Sesink, A.L., et al., Red meat and colon cancer: the cytotoxic and hyperproliferative effects of dietary heme. Cancer Res, 1999. 59(22): p. 5704-9.

69. Sesink, A.L.A., et al., Red meat and colon cancer: Dietary haem, but not fat, has cytotoxic and hyperproliferative effects on rat colonic epithelium. Carcinogenesis, 2000. 21: p. 1909-1915.

70. IJssennagger, N., et al., Dietary haem stimulates epithelial cell turnover by downregulating feedback inhibitors of proliferation in murine colon. Gut, 2012. 61(7): p. 1041-9.

71. Hu, F.B., Metabolic profiling of diabetes: from black-box epidemiology to systems epidemiology. Clin Chem, 2011. 57(9): p. 1224-6.

72. Wild, C., P. Vineis, and S. Garte, Molecular Epidemiology of Chronic Disease. 2008, West Sussex, England: John Wiley \& Soncs Ltd.

73. Shimada, T., Xenobiotic-metabolizing enzymes involved in activation and detoxification of carcinogenic polycyclic aromatic hydrocarbons. Drug Metab Pharmacokinet, 2006. 21(4): p. 257-76.

74. Xue, W. and D. Warshawsky, Metabolic activation of polycyclic and heterocyclic aromatic hydrocarbons and DNA damage: a review. Toxicol Appl Pharmacol, 2005. 206(1): p. 73-93.

75. Kampman, E., et al., Meat consumption, genetic susceptibility, and colon cancer risk: a United States multicenter case-control study. Cancer Epidemiol Biomarkers Prev, 1999. 8(1): p. 15-24.

76. Le Marchand, L., et al., Red meat intake, CYP2E1 genetic polymorphisms, and colorectal cancer risk. Cancer Epidemiol Biomarkers Prev, 2002. 11(10 Pt 1): p. 1019-24.

77. Roberts-Thomson, I.C., et al., Diet, acetylator phenotype, and risk of colorectal neoplasia. Lancet, 1996. 347(9012): p. 1372-4.

78. Koutros, S., et al., Xenobiotic metabolizing gene variants, dietary heterocyclic amine intake, and risk of prostate cancer. Cancer Res, 2009. 69(5): p. 1877-84.

79. Sharma, S., et al., Well-done meat consumption, NAT1 and NAT2 acetylator genotypes and prostate cancer risk: the multiethnic cohort study. Cancer Epidemiol Biomarkers Prev, 2010. 19(7): p. 1866-70. 
80. Lee, H.J., et al., Polymorphisms in Xenobiotic Metabolizing Genes, Intakes of Heterocyclic Amines and Red Meat, and Postmenopausal Breast Cancer. Nutr Cancer, 2013.

81. Mignone, L.I., et al., Meat consumption, heterocyclic amines, NAT2, and the risk of breast cancer. Nutr Cancer, 2009. 61(1): p. 36-46.

82. Thier, R., et al., Markers of genetic susceptibility in human environmental hygiene and toxicology: the role of selected CYP, NAT and GST genes. Int J Hyg Environ Health, 2003. 206(3): p. 149-71.

83. van den Brandt, P.A., et al., A large-scale prospective cohort study on diet and cancer in The Netherlands. J Clin Epidemiol, 1990. 43(3): p. 285-95. 



\title{
CHAPTER 2
}

\section{Meat consumption and changes in BMI over time: longitudinal analyses from the Netherlands Cohort Study}

\author{
Anne M.J. Gilsing \\ Matty P. Weijenberg \\ Laura A.E. Hughes \\ Ton Ambergen \\ Pieter C. Dagnelie \\ R. Alexandra Goldbohm \\ Piet A. van den Brandt \\ and Leo J. Schouten
}

Adapted from the Journal of Nutrition 2012;142(2):340-9 


\section{ABSTRACT}

Introduction: Hypotheses regarding the role of meat consumption in body weight modulation are contradictory. Prospective studies on an association between meat consumption and BMI change are limited.

Methods: We assessed the association between meat consumption and change in BMI over time in 3902 men and women aged 55-69 y from the Netherlands Cohort Study. Dietary intake was estimated at baseline by a food-frequency questionnaire. BMI was ascertained through baseline self-reported height (1986) and weight (1986, 1992 and 2000). Analyses were based on sex-specific categories of daily total fresh meat, red meat, beef, pork, minced meat, chicken, processed meat and fish consumption at baseline. Linear mixed effect modelling adjusted for confounders was used to assess longitudinal associations.

Results: Significant cross-sectional differences in BMI between quintiles of total meat intake were observed ( $P$-trend $<0.01$; both sexes). No association between total fresh meat consumption and prospective $\mathrm{BMI}$ change was observed in men and women $(\triangle \mathrm{BMI}$ change highest vs lowest quintile after $14 \mathrm{y}$ : men $-0.06 \mathrm{~kg} / \mathrm{m}^{2}, P=0.75$; women $0.26 \mathrm{~kg} / \mathrm{m}^{2}, P=0.20$ ). Men with the highest intake of beef experienced a significantly lower increase in BMI after 6 and $14 \mathrm{y}$ than those with the lowest intake. After $14 \mathrm{y}$, a higher increase in BMI was associated with higher intakes of chicken in women and with pork in both sexes. Results remained similar when stratifying on median baseline BMI, and age-stratified analyses yielded mixed results.

Discussion: Meat consumption, or factors directly related to meat intake, is not strongly associated with weight change during 14 year prospective follow-up in this elderly population. 


\section{INTRODUCTION}

Overweight and obesity have reached pandemic proportions resulting in a growing global burden of obesity related chronic disease $[1,2]$. Hence, there is an increasing interest to identify modifiable factors in the diet that may be associated with maintaining a healthy body weight. In this respect, several plausible hypotheses underlie a potential role for meat consumption in the development of adiposity.

Cross-sectional research has shown considerable lower obesity rates among vegetarians compared to meat-eaters in Western populations [3-7]. However, whether these differences in body weight can be explained by the abstinence of meat, the higher intake of beneficial dietary components such as fruit and vegetables, the overall lower energy intake, or the more favorable non dietary lifestyle factors of vegetarians has been debated. Nonetheless, cross-sectional studies examining the effect of meat consumption in particular on body weight have yielded ambiguous results [8-11], but are above all limited by their inability to distinguish between cause and effect.

Until now, two longitudinal studies investigated the possible role of vegetarianism in preventing weight gain. Rosell et al. [12] observed only small differences in weight gain between meat eating and vegetarian men over a 5 year period, whereas a smaller study in Seventh-day Adventists [13] showed non-significant and mixed results. The few studies which prospectively examined the role of dietary meat consumption for weight maintenance yielded mixed results [14-21] and mostly reported only on the effects of total meat intake and not on different sources and types of meat products.

In contrast, high protein diets have also been advocated for weight reduction and body weight regulation. Meat products are generally a rich source of high quality proteins which may increase satiety and up regulate thermogenesis [22]. Although well-designed long term studies are lacking, high protein diets have shown to positively affect weight maintenance on the short term [23]. In addition, studies on the weight reducing potential of the specific high protein, low carbohydrate Atkins diet [24], containing ad libitum amounts of meat, have been inconsistent [25-28].

The Netherlands Cohort Study on diet and cancer (NLCS) acquired data from up to three consecutive weight measurements over a period of 14 years for 5,000 elderly individuals who were randomly selected from the total cohort. This study provides the unique opportunity to examine the association between the dietary intake of fresh meat, processed meat, poultry and fish and subsequent BMI change over time in the general elderly population.

\section{METHODS}

\section{Subjects and study design}

The Netherlands Cohort Study was conceived in September 1986 with the enrolment of 120,852 individuals in the Netherlands and with the purpose to investigate the associations between diet and the development of cancer. The cohort comprised 58279 men and 62573 women aged 55-69 years at baseline who completed a self-administered questionnaire on dietary habits, lifestyle, medical history, and demographic information. Municipal registries 
throughout the Netherlands were used to constitute an effective sampling frame of the population. Full details of the study design have been described elsewhere [29].

The NLCS is a case-cohort design with a subcohort of 5000 individuals randomly selected from the larger cohort on recruitment into the study. These individuals have been followed-up biennially from baseline in 1986 for migration and vital status to estimate person time at risk. Follow-up of the subcohort has also allowed for the additional accumulation of prospective data regarding a number of factors related to body weight and weight change. This subcohort was the study population used for the present study.

For the current analyses, individuals who returned incomplete baseline dietary questionnaires, individuals with missing values for height or weight at baseline, and all prevalent cancer cases other than skin cancer at baseline were excluded. This resulted in an initial study population of 4280 individuals. Follow-up measurements regarding BMI were recorded in the years 1992 and 2000, and data were available for 3787 and 2091 individuals, respectively, at these time points. The NLCS has been approved by the institutional review boards of the CIVO-TNO Research Institute (Zeist, the Netherlands) and Maastricht University (Maastricht, the Netherlands). The Institutional Review Board of Maastricht University approved the analyses reported herein.

\section{Dietary assessment}

Habitual meat intake at baseline was assessed using a self-administered semi-quantitative food-frequency questionnaire (FFQ) consisting of 150 food-items, estimating the average frequency of foods and beverages consumed over the previous 12 months. The questionnaire contained 14 items on the consumption of meat with the hot meal (mainly fresh meat, including chicken), 5 items on the consumption of meat products used as sandwich fillings, and 3 items on fish consumption. This dietary assessment was part of a larger questionnaire that included questions regarding several lifestyle factors.

Fresh meat consisted of beef, pork, minced meat (including beef and pork), chicken, liver, and other meat (e.g. horsemeat, lamb). Fresh red meat consisted of the fresh meat items minus the chicken item. Coding of fresh meat items was based on raw weight to take into account the amount of fat originally present in the meat but eventually ending up in the gravy, which is usually consumed as well. Processed meat was defined as meat items that had undergone some form of preservation (mostly treatment with nitrate salt, some types smoked or fermented). The meat intake in each participant's diet was calculated from the FFQ dietary data by summing the multiplied frequencies and serving sizes of the meat items.

\section{Ascertainment of BMI}

Subjects in the subcohort were followed up biennially since the time of the baseline questionnaire. At these time points, additional self-administered questionnaires were mailed to all individuals given they were still alive and reachable at the same address. If necessary, new addresses were obtained through the municipal population registries and individuals were contacted there. Height $(\mathrm{cm})$ was reported on the baseline questionnaire. Body weight $(\mathrm{kg})$ was reported on questionnaires on three different occasions: at baseline in 1986, in 1992 and 2000. In 1986 and 1992 the question was open-ended and phrased as 
"how much do you weigh now (kg)?" In 2000, subjects were asked "have you weighed yourself recently (yes/no)?" If subjects answered "yes", they were directed to an open-end question, "how much do you weigh $(\mathrm{kg})$ ?" At all three time points, the weight measurement was self-reported. BMI $\left(\mathrm{kg} / \mathrm{m}^{2}\right)$ was calculated for each time point by using the recorded weight at each time point divided by height squared at baseline. Moreover, the baseline questionnaire included the open-end question, "what was your weight at age $20(\mathrm{~kg})$ ?", and a corresponding BMI was calculated using the recorded weight at age 20 divided by height squared at baseline (age 55-69 y). An overview of the study design is presented in Supplemental Figure 1.

\section{Statistical analyses}

Data were analyzed using Stata (Intercooled STATA, version 11; Stata-Corp LP, College Station, TX). All analyses were conducted separately for different types of meat intake and were stratified by sex.. Total fresh meat consumption was quantified using two parameters; quintiles of intake (in $\mathrm{g} / \mathrm{d}$ ) and weekly frequency of meat intake (0-3d/wk, 4-5d/wk, 6$7 d / w k)$. Sex-specific intakes of total fresh meat, weekly meat consumption frequency, fresh red meat, beef, pork, minced meat, chicken, processed meat and fish were assessed. Mean total meat and individual meat product intake as well as other population characteristics were analyzed using data from the baseline questionnaire. Pearson's correlation was applied to determine the association between continuous baseline characteristics and total fresh meat consumption. Cross sectional differences in baseline BMI values per quintile of total fresh meat consumption were examined by using a linear regression model adjusted for confounding variables selected for the longitudinal analyses as described below.

Linear mixed effect (LME) modelling was applied to assess the change in BMI over time and the longitudinal relation between meat consumption intake at baseline and BMI at baseline (1986), in 1992 and in 2000. The mixed model consists of two parts: fixed effects and random effects. Fixed effects describe population slopes for a set of considered covariates, which include exposures and confounders. Random effects describe individual variability in outcome and in changes over time. We considered a model with individual random slopes for time. The model allowed us to examine the influence of covariates on the change in BMI over time [30]. This model also accounts for the correlation between repeated measures and accounts for missing values at different time points [31].

For the LME model, a categorical time variable was created by using the values 0,6 and 14 reflecting the time points (in years) of the BMI measurement from baseline. Two dummy variables indicating the three time points and 4 meat intake dummy variables (reflecting quintiles or 5 categories of intake) were entered into the model. In case of chicken and fish consumption 3 dummy variables were entered, and for reported weekly frequency of meat intake 2 dummies were created, (reflecting the 4 or 3 categories of intake, respectively). Furthermore, interactions of the time dummies with the meat intake dummies $(8,6$ or 4 interaction terms, respectively) were included. A random slope for time at the individual level was obtained by using time as a continuous variable. Longitudinal information was derived by considering the regression coefficients of the time dummies and the regression coefficients of the meat-time interaction terms. The coefficients of the time dummies reflect the change in BMI over time (6 and $14 \mathrm{y}$, respectively) in the lowest quintile/category. The coefficient of an interaction term gives the difference between the 
change in BMI in a quintile/category after 6 and $14 \mathrm{y}$ and the change in BMI in the lowest quintile/category of meat intake as the reference category. All models were examined for evidence of an overall trend by statistically testing whether any of the time-by-quintile interaction-terms is equal to zero. This provides an overall assessment as to whether the increase in BMI over time is different between the quintiles/categories of intake; if significant; the direction of this trend should be quantified based on the individual coefficients of interaction.

In addition to an unadjusted and age adjusted model, we created an LME model adjusted for age plus confounding variables. These confounding variables were identified as being associated with both $\mathrm{BMI}$ and meat intake from the previous literature or were variables that, when entered in our model, resulted in a $>10 \%$ change in the regression coefficients. These include age (years), total energy intake (kcal), non-occupational physical activity ( $\mathrm{min} / \mathrm{d})$, smoking status (never-, ex- and current smoker), education (low, medium, high), alcohol intake (g/d), grain and grain product consumption (e.g. rice, cereals, oats, noodles) (g/day), fruit consumption (g/d), and vegetable consumption $(\mathrm{g} / \mathrm{d})$. We additionally adjusted for special dieting habits (on doctor's advice) in the 5 y preceding the baseline questionnaire (yes/no). To additionally examine the independent contribution of the individual meat categories (total fresh meat, fresh red meat, beef, pork, minced meat, chicken and liver), a fourth model was created in which the complementary meat items were also included in the respective multivariable models. To enable comparison, the age and energy-adjusted analyses were restricted to subjects included in multivariable-adjusted analyses (e.g., with no missing values on confounding variables; $n=3902$ ).

Although LME models should be robust to missing values, we conducted the initial analysis excluding individuals with missing BMI at 1 or 2 time points $(n=1855$ remaining). To evaluate potential bias linked to diet modification (related to disease) during follow-up, we excluded individuals who developed cancer between 1986 and 2000 ( $n=2878$ remaining). We investigated for all longitudinal analyses whether an association was present both in individuals with a high and low BMI at baseline by performing stratified analyses using median BMI at baseline (women: 24.49; men: 24.76) as cut-points. We also examined whether similar associations were found across the age spectrum of our subcohort by stratifying our initial analyses in three age categories (54-59, 60-64, and 65-70 y).

In addition, we compared the prospective results to the association between meat consumption and change in BMI between age 20 and baseline, assuming that meat consumption at baseline reflects that during adulthood. This secondary analysis was performed using multiple linear regression, in which mean BMI change (age 20-baseline) (and $95 \% \mathrm{Cl}$ ) were derived, adjusted for all confounders from the LME models and BMI at age 20. Trends were evaluated with the Wald test by assigning participants the median value for each level of the categorical exposure variables and this variable was entered as a continuous term in the regression model. All tests were two-tailed and differences were regarded as statistically significant at $P<0.05$ in all analyses. 


\section{RESULTS}

Baseline population characteristics are presented in Table 1. Meat consumption was higher in men than in women for all investigated meat categories with the exception of chicken intake which was similar among both sexes. Compared with women, men consumed more calories, drank more alcohol and had a higher proportion of former and current smokers. For both men and women, higher total fresh meat consumption was correlated with higher total energy intake, a younger age, a higher BMI and a higher consumption of alcohol. In contrast to men, body height was not correlated with total fresh meat consumption in women.

Table 1: Baseline characteristics of the subcohort in the Netherlands Cohort Study

\begin{tabular}{|c|c|c|c|c|c|c|}
\hline \multirow[b]{2}{*}{ Characteristics } & \multicolumn{3}{|c|}{ Men $(N=1991)$} & \multicolumn{3}{|c|}{ Women ( $N=1911)$} \\
\hline & & $\begin{array}{l}\text { Correlation } \\
\text { with total fresh } \\
\text { meat intake }^{1,2}\end{array}$ & $P^{3}$ & & $\begin{array}{l}\text { Correlation } \\
\text { with total fresh } \\
\text { meat intake }\end{array}$ & $P^{3}$ \\
\hline Age, y & $\pm 4.2^{4}$ & -0.06 & $<0.05$ & \pm 4.3 & -0.04 & 0.12 \\
\hline \multicolumn{7}{|l|}{ Anthropometrics } \\
\hline Height, cm & $176.6 \pm 6.6$ & 0.06 & $<0.05$ & $165.2 \pm 6.1$ & -0.01 & 0.98 \\
\hline Weight, kg & $77.7 \pm 9.4$ & 0.15 & $<0.05$ & $68.2 \pm 10.0$ & 0.18 & $<0.05$ \\
\hline $\mathrm{BMI}, \mathrm{kg} / \mathrm{m}^{2}$ & \pm 2.5 & 0.14 & $<0.05$ & \pm 3.5 & 0.19 & $<0.05$ \\
\hline \multicolumn{7}{|l|}{ Dietary factors } \\
\hline $\begin{array}{l}\text { Total energy intake, } \mathrm{kcal} / \mathrm{d} \\
\text { Types of meat and fish, } \mathrm{g} / \mathrm{d}\end{array}$ & $2170 \pm 503$ & 0.22 & $<0.05$ & $1690 \pm 391$ & 0.18 & $<0.05$ \\
\hline Total fresh meat ${ }^{5}$ & \pm 42.6 & - & - & \pm 39.3 & - & - \\
\hline Fresh red meat ${ }^{6}$ & \pm 41.0 & 0.93 & $<0.05$ & \pm 37.8 & 0.92 & $<0.05$ \\
\hline Beef & \pm 23.9 & 0.45 & $<0.05$ & \pm 21.6 & 0.41 & $<0.05$ \\
\hline Pork & \pm 30.1 & 0.62 & $<0.05$ & \pm 28.1 & 0.65 & $<0.05$ \\
\hline Minced meat ${ }^{7}$ & \pm 17.7 & 0.44 & $<0.05$ & \pm 15.0 & 0.40 & $<0.05$ \\
\hline Chicken & \pm 14.5 & 0.30 & $<0.05$ & \pm 15.9 & 0.31 & $<0.05$ \\
\hline Processed meat & \pm 17.0 & 0.13 & $<0.05$ & \pm 11.6 & 0.17 & $<0.05$ \\
\hline Fish & \pm 16.8 & 0.01 & 0.72 & \pm 13.9 & 0.01 & 0.76 \\
\hline \multicolumn{7}{|l|}{ Lifestyle behaviours } \\
\hline \multicolumn{7}{|l|}{ Cigarette smoking status, \% } \\
\hline Never & 12.9 & - & - & 56.5 & - & - \\
\hline Former & 51.8 & - & - & 21.8 & - & - \\
\hline Current & 35.4 & - & - & 21.8 & - & - \\
\hline Alcohol Intake, g/day & \pm 17.1 & & & $6.0 \pm 9.7$ & & \\
\hline \multicolumn{7}{|l|}{ Physical Activity, \% } \\
\hline$<30 \mathrm{~min} / \mathrm{d}$ & 17.5 & - & - & 22.8 & - & - \\
\hline $30-60 \mathrm{~min} / \mathrm{d}$ & 31.1 & - & - & 31.2 & - & - \\
\hline $60-90 \mathrm{~min} / \mathrm{d}$ & 19.4 & - & - & 23.4 & - & - \\
\hline$>90 \mathrm{~min} / \mathrm{d}$ & 32.0 & - & - & 22.6 & - & - \\
\hline \multicolumn{7}{|c|}{$\begin{array}{l}{ }^{1} \text { Reported as pairwise Pearson correlation coefficients. } \\
{ }^{2} \text { Including all types of meat (except processed meat) and poultry. } \\
{ }^{3} \text { P-values for pairwise correlation coefficients. } \\
{ }^{4} \text { Mean } \pm \text { SD (all such values). } \\
{ }^{5} \text { Including all types of meat (except processed meat) and poultry. } \\
6 \text { Including beef, pork, minced meat, liver and other meat. } \\
{ }^{7} \text { Including beef and pork. }\end{array}$} \\
\hline
\end{tabular}


Statistically significant cross sectional differences in BMI at baseline were observed between quintiles of total fresh meat consumption in men but most predominantly in women (Table 2). In both sexes, those with the highest meat intake also had the highest $\mathrm{BMI}$ at baseline. Although $\mathrm{BMI}$ did not change over time in men, $\mathrm{BMI}$ in women increased with $0.19 \mathrm{~kg} / \mathrm{m}^{2}$ and $0.42 \mathrm{~kg} / \mathrm{m}^{2}$ from baseline to 6 and $14 \mathrm{y}$ of follow- up, respectively $(P<0.05)$ (Table 3).

Table 2: Baseline BMI per quintile of total fresh meat consumption in the subcohort of the Netherlands Cohort Study ${ }^{1}$

\begin{tabular}{|c|c|c|c|c|c|c|}
\hline \multirow[b]{2}{*}{$\begin{array}{l}\text { Quintile of total fresh meat } \\
\text { consumption }\end{array}$} & \multicolumn{3}{|c|}{$\operatorname{Men}(N=1991)^{2}$} & \multicolumn{3}{|c|}{ Women $(N=1911)^{2}$} \\
\hline & $\mathrm{N}$ & $\begin{array}{l}\mathrm{BMI} \\
\left(\mathrm{kg} / \mathrm{m}^{2}\right)\end{array}$ & $(95 \% \mathrm{Cl})$ & $\mathrm{N}$ & $\begin{array}{l}\mathrm{BMI} \\
\left(\mathrm{kg} / \mathrm{m}^{2}\right)\end{array}$ & $(95 \% \mathrm{Cl})$ \\
\hline 1 & 395 & 24.5 & $(24.3-24.7)$ & 389 & 24.1 & $(23.8-24.4)$ \\
\hline 2 & 401 & 24.7 & $(24.6-24.9)$ & 371 & 24.7 & $(24.5-24.8)$ \\
\hline 3 & 406 & 24.9 & $(24.8-25.0)$ & 387 & 25.0 & $(24.8-25.1)$ \\
\hline 4 & 394 & 25.0 & $(24.9-25.2)$ & 392 & 25.3 & $(25.1-25.4)$ \\
\hline 5 & 395 & 25.4 & $(25.2-25.6)$ & 372 & 26.0 & $(25.7-25.2)$ \\
\hline$P$-trend & & & $<0.001$ & & & $<0.001$ \\
\hline
\end{tabular}

${ }^{1}$ All values are mean $\pm 95 \% \mathrm{Cl}$. Results derived from a linear regression model adjusted for mean values of age, baseline total energy intake $(\mathrm{kcal})$, alcohol intake $(\mathrm{g} / \mathrm{d})$, vegetable consumption $(\mathrm{g} / \mathrm{d})$, fruit consumption $(\mathrm{g} / \mathrm{d})$, consumption of grains $(\mathrm{g} / \mathrm{d})$, physical activity level, smoking status, level of education, dieting habits in the past $5 \mathrm{y}$. ${ }^{2}$ Number of participants at baseline.

${ }^{3}$ Range of total meat consumption (g/d) men: Q1, 574 ; 22, 75-93; Q3, 94-108; Q4, 109-136; Q5, 137-296; Range of total meat consumption (g/d) women: Q1, $\leq 62 ; \mathrm{Q} 2,63-84 ; \mathrm{Q} 3,85-101 ; \mathrm{Q} 4,102-122 ; \mathrm{Q} 5,123-280$.

Table 3: BMI at baseline (1986), in 1992, and 2000 in subcohort members of the Netherlands Cohort Study ${ }^{1,2}$

\begin{tabular}{|c|c|c|c|c|c|c|c|c|}
\hline \multirow[b]{2}{*}{ Year } & \multicolumn{4}{|l|}{ Men } & \multicolumn{4}{|c|}{ Women } \\
\hline & $\mathrm{N}$ & $\mathrm{BM}$ & & & $\mathrm{N}$ & BMI & & \\
\hline 1986 & 1991 & 24.9 & \pm & 0.06 & 1911 & 25.0 & \pm & 0.08 \\
\hline 1992 & 1692 & 25.0 & \pm & 0.06 & 1678 & 25.2 & \pm & $0.08^{a}$ \\
\hline 2000 & 938 & 25.0 & \pm & 0.08 & 958 & 25.4 & \pm & $0.10^{\mathrm{a}}$ \\
\hline
\end{tabular}

${ }^{1}$ All values are mean $\pm \mathrm{SE}$.

${ }^{2} \mathrm{BMI}$ derived from a linear mixed model including BMI and time.

${ }^{\text {a }}$ Different from baseline, $P<0.05$.

The course of BMI change from baseline until 14 y of follow-up adjusted for confounding variables is depicted by quintiles of total fresh meat consumption (Figure 1) and frequency of meat consumption (Figure 2). The BMI of men increased only modestly after $14 \mathrm{y}$ from baseline over all categories and quintiles of intake and no clear association between quintiles of intake and BMI change was observed. In women BMI increased in each quintile and frequency of intake over time, with a tendency for the highest increase in those with the highest meat consumption. Nonetheless, no statistically significant differences between quintiles and frequencies of intake or any significant trends were observed.

The association between BMI change between baseline and 6 and $14 \mathrm{y}$ of follow-up and specific subtypes of meat was examined by 4 different models (unadjusted, age-adjusted, confounders-adjusted, and adjusted for confounders and complementary meat items) which yielded comparable results. Table $4 \mathrm{~A}$ and Table 4B present the findings for the confounder-adjusted model stratified for men and women respectively, according to quintiles and categories of intake and can be interpreted as the change in BMI in each 
quintile/category at a given follow-up time compared to baseline. Men with the highest beef intake had a significantly lower increase in BMI than did men in the lowest quintile both after 6 and 14 y and a borderline significant trend was observed $(P=0.06)$. Women in the highest quintile of pork consumption had a significantly higher increase in BMI than did women in the lowest quintile after 14 y $(P=0.02)$. In addition, women and men with the highest chicken consumption had a significantly higher increase in $\mathrm{BMI}$ compared to those with the lowest intake after 14 y (men $P=0.03$; women $P=0.05$ ).
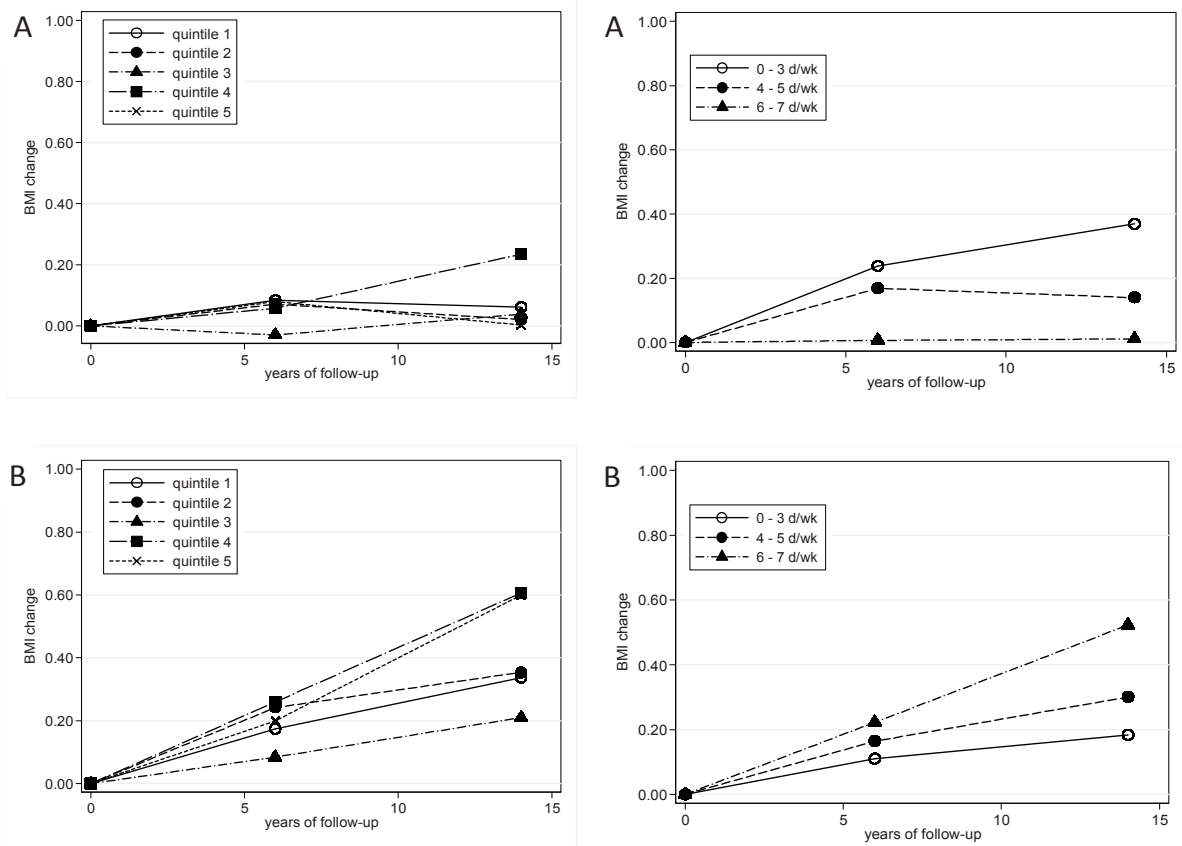

Figure 1: Change in BMI $\left(\mathrm{kg} / \mathrm{m}^{2}\right)$ over $14 \mathrm{y}$ in each quintile of baseline total fresh meat intake in A) men $(P$-trend $=0.70)$ and $\mathrm{B})$ women $(P$-trend $=0.46)$. Graphs derived from linear mixed models adjusted for age $(\mathrm{y})$, total energy intake $(\mathrm{kcal} / \mathrm{d})$, physical activity level $(<30,30-60,60-90,>90 \mathrm{~min} / \mathrm{d})$, smoking status (never, former and current smoker), alcohol consumption (g/d), level of education (low, medium, high), vegetable consumption (g/d), fruit consumption $(\mathrm{g} / \mathrm{d})$, consumption of grains $(\mathrm{g} / \mathrm{d})$ and special dieting habits (yes or no to being on a diet in the past $5 \mathrm{y}$ ). Total meat includes all types of meat (except processed meat) and poultry. $P$-trend tests for overall difference in BMI increase over time between groups of meat intake. Range of total meat consumption (g/d) in men: Q1 $\leq 74$ (; Q2, 75-93; Q3, 94-108; Q4, 109136; Q5, 137-296; in women Q1, $\leq 62 ; \mathrm{Q} 2,63-84 ; \mathrm{Q} 3$,

Figure 2: Change in $\mathrm{BMI}\left(\mathrm{kg} / \mathrm{m}^{2}\right)$ over $14 \mathrm{y}$ in each category of self-reported frequency of meat consumption in A) men ( $P$-trend $=0.17$ ) and $\mathrm{B}$ ) women $(P$-trend $=0.40)$. Graphs derived from linear mixed models adjusted for age (y), total energy intake (kcal/d), physical activity level $(<30,30-60,60-90,>90$ $\mathrm{min} / \mathrm{d}$ ), smoking status (never, former and current smoker), alcohol consumption (g/d), level of education (low, medium, high), vegetable consumption $(g / d)$, fruit consumption $(g / d)$, consumption of grains $(\mathrm{g} / \mathrm{d})$ and special dieting habits (yes or no to being on a diet in the past $5 \mathrm{y}$ ). Total meat includes all types of meat (except processed meat) and poultry. $P$-trend tests for overall difference in $\mathrm{BMI}$ increase over time between groups of meat intake. 
Table 4A: Change in BMI from baseline (1986) over time associated with specific dietary meat intakes by quintile (Q) or categories (C) of meat intake for male subcohort members $(n=1991)$ of the Netherlands Cohort Study ${ }^{1}$

\begin{tabular}{|c|c|c|c|c|c|c|c|c|c|c|c|c|}
\hline & & \multicolumn{10}{|c|}{ Quintile or category of meat intake ${ }^{2}$} & \multirow[b]{2}{*}{$\begin{array}{l}P- \\
\text { trend }\end{array}$} \\
\hline & & 1 & & 2 & & 3 & & 4 & & 5 & & \\
\hline \multicolumn{13}{|l|}{ Fresh red meat ${ }^{4}, \mathrm{~g} / \mathrm{d}$} \\
\hline & 1992 & 0.15 & \pm 0.08 & 0.12 & \pm 0.13 & -0.03 & \pm 0.12 & 0.04 & \pm 0.12 & 0.01 & \pm 0.12 & \\
\hline & 2000 & 0.23 & \pm 0.13 & 0.13 & \pm 0.11 & 0.06 & \pm 0.11 & 0.08 & \pm 0.11 & -0.11 & \pm 0.11 & 0.59 \\
\hline \multicolumn{13}{|l|}{ Beef, g/d } \\
\hline & 1992 & 0.18 & \pm 0.08 & 0.11 & \pm 0.12 & 0.09 & \pm 0.13 & -0.21 & \pm 0.12 & -0.09 & $\pm 0.13^{a}$ & \\
\hline & 2000 & 0.33 & \pm 0.13 & 0.12 & \pm 0.11 & 0.19 & \pm 0.11 & -0.01 & \pm 0.11 & -0.27 & $\pm 0.11^{a}$ & 0.06 \\
\hline \multicolumn{13}{|l|}{ Pork, g/d } \\
\hline & 1992 & 0.15 & \pm 0.08 & 0.03 & \pm 0.12 & 0.06 & \pm 0.13 & 0.08 & \pm 0.12 & -0.06 & \pm 0.12 & \\
\hline & 2000 & -0.04 & \pm 0.13 & -0.01 & \pm 0.11 & 0.14 & \pm 0.11 & 0.12 & \pm 0.11 & 0.11 & \pm 0.11 & 0.39 \\
\hline \multicolumn{13}{|l|}{ Minced meat ${ }^{5}, \mathrm{~g} / \mathrm{d}$} \\
\hline & 1992 & 0.03 & \pm 0.08 & 0.01 & \pm 0.13 & -0.07 & \pm 0.12 & 0.07 & \pm 0.12 & 0.22 & \pm 0.21 & \\
\hline & 2000 & 0.18 & \pm 0.13 & 0.06 & \pm 0.11 & -0.08 & \pm 0.11 & 0.24 & \pm 0.11 & -0.02 & \pm 0.11 & 0.05 \\
\hline \multicolumn{13}{|c|}{ Processed meat, $\mathrm{g} / \mathrm{d}$} \\
\hline & 1992 & 0.15 & \pm 0.08 & 0.07 & \pm 0.13 & -0.05 & \pm 0.12 & 0.01 & \pm 0.12 & 0.07 & \pm 0.12 & \\
\hline & 2000 & 0.07 & \pm 0.13 & 0,00 & \pm 0.11 & 0.19 & \pm 0.11 & 0.07 & \pm 0.11 & 0.02 & \pm 0.11 & 0.41 \\
\hline \multicolumn{13}{|l|}{ Chicken, g/d } \\
\hline & 1992 & 0.02 & \pm 0.07 & -0.02 & \pm 0.11 & 0.05 & \pm 0.11 & 0.13 & \pm 0.10 & - & & \\
\hline & 2000 & -0.17 & \pm 0.12 & -0.09 & \pm 0.10 & 0.29 & $\pm 0.10^{b}$ & 0.19 & $\pm 0.09^{b}$ & - & & 0.04 \\
\hline \multicolumn{13}{|l|}{ Fish, g/d } \\
\hline & 1992 & -0.03 & \pm 0.07 & -0.01 & \pm 0.12 & 0.09 & \pm 0.10 & 0.14 & \pm 0.12 & - & & \\
\hline & 2000 & -0.03 & \pm 0.11 & -0.06 & \pm 0.10 & 0.12 & \pm 0.09 & 0.28 & \pm 0.11 & - & & 0.39 \\
\hline
\end{tabular}

${ }^{1}$ All values are mean \pm SE. Results derived form a linear mixed model adjusted for age, baseline total energy intake (kcal), physical activity level $(<30,30-60,60-90,>90 \mathrm{~min} / \mathrm{d})$, smoking status (never, former and current smoker), alcohol intake (g/d), level of education (low, medium, high), vegetable consumption (g/d), fruit consumption (g/d), consumption of grains $(\mathrm{g} / \mathrm{d}$ ) and dieting habits (yes or no to being on a diet in the past $5 \mathrm{y}$ ).

${ }^{2}$ Range of total fresh meat consumption (g/d): Q1, $\leq 75 ;$ Q2, 745-94; Q3, 94-108; Q4, 108-137; Q5, 137-296.

${ }^{3}$ Test for overall difference in BMI increase over time between groups of meat intake.

${ }^{4}$ Including beef, pork, minced meat, liver and other meat.

${ }^{5}$ Including beef and pork.

${ }^{a}$ Significantly lower BMI increase than in the lowest quintile of intake, as derived from the quintile-by-time interaction $(P<0.05)$.

${ }^{\mathrm{b}}$ Significantly higher BMI increase than in the lowest quintile of intake, as derived from the quintile-by-time interaction $(P<0.05)$.

When stratifying our data on median BMI at baseline, we observed virtually similar associations between (subtypes) of meat intake and changes in BMI (results not shown). Our age stratified (54-59, 60-64, 65-70 y) analyses yielded mixed results for several subtypes of meat, possibly due to smaller numbers in the age categories (results not shown).

When excluding subjects with missing BMI measurements at 1 or 2 time points, results attenuated slightly for beef, pork and chicken, although the observed pattern across quintiles remained similar. Other results did not change noticeably. Our findings did not change when individuals who developed cancer after 1986 were excluded (data not shown). 
Table 4B: Change in BMI from baseline (1986) over time associated with specific dietary meat intakes by quintile (Q) or categories (C) of meat intake for female subcohort members $(n=1911)$ of the Netherlands Cohort Study ${ }^{1}$

\begin{tabular}{|c|c|c|c|c|c|c|c|c|c|c|c|c|}
\hline & & \multicolumn{10}{|c|}{ Quintile or category of meat intake ${ }^{2}$} & \multirow[b]{2}{*}{$\begin{array}{l}P_{-} \\
\text {trend }\end{array}$} \\
\hline & & 1 & & 2 & & 3 & & 4 & & 5 & & \\
\hline \multicolumn{13}{|l|}{ Fresh red meat ${ }^{4}, \mathrm{~g} / \mathrm{d}$} \\
\hline & 1992 & 0.18 & \pm 0.09 & -0.25 & \pm 0.14 & 0.22 & \pm 0.14 & 0.09 & \pm 0.14 & 0.23 & \pm 0.14 & \\
\hline & 2000 & 0.26 & \pm 0.14 & 0.42 & \pm 0.12 & 0.50 & \pm 0.12 & 0.37 & \pm 0.12 & 0.55 & \pm 0.12 & 0.79 \\
\hline \multicolumn{13}{|l|}{ Beef, g/d } \\
\hline & 1992 & 0.13 & \pm 0.09 & 0.33 & \pm 0.14 & 0.33 & \pm 0.14 & 0.06 & \pm 0.14 & 0.10 & \pm 0.14 & \\
\hline & 2000 & 0.39 & \pm 0.15 & 0.44 & \pm 0.12 & 0.58 & \pm 0.12 & 0.41 & \pm 0.12 & 0.24 & \pm 0.13 & 0.16 \\
\hline \multicolumn{13}{|l|}{ Pork, g/d } \\
\hline & 1992 & 0.21 & \pm 0.09 & 0.22 & \pm 0.15 & 0.22 & \pm 0.14 & 0.09 & \pm 0.14 & 0.23 & \pm 0.14 & \\
\hline & 2000 & 0.13 & \pm 0.14 & 0.52 & \pm 0.13 & 0.46 & \pm 0.12 & 0.39 & \pm 0.12 & 0.60 & $\pm 0.13^{b}$ & 0.29 \\
\hline \multicolumn{13}{|l|}{ Minced meat ${ }^{5}, \mathrm{~g} / \mathrm{d}$} \\
\hline & 1992 & 0.14 & \pm 0.09 & 0.12 & \pm 0.14 & 0.19 & \pm 0.14 & 0.30 & \pm 0.14 & 0.21 & \pm 0.15 & \\
\hline & 2000 & 0.43 & \pm 0.15 & 0.44 & \pm 0.12 & 0.36 & \pm 0.12 & 0.46 & \pm 0.12 & 0.41 & \pm 0.13 & 0.88 \\
\hline \multicolumn{13}{|c|}{ Processed meat, $\mathrm{g} / \mathrm{d}$} \\
\hline & 1992 & 0.13 & \pm 0.09 & 0.13 & \pm 0.14 & 0.27 & \pm 0.15 & 0.34 & \pm 0.14 & 0.10 & \pm 0.14 & \\
\hline & 2000 & 0.28 & \pm 0.14 & 0.44 & \pm 0.12 & 0.46 & \pm 0.13 & 0.61 & \pm 0.12 & 0.29 & \pm 0.12 & 0.51 \\
\hline \multicolumn{13}{|l|}{ Chicken, g/d } \\
\hline & 1992 & 0.04 & \pm 0.08 & 0.28 & $\pm 0.12^{b}$ & 0.16 & \pm 0.13 & 0.26 & \pm 0.12 & - & & \\
\hline & 2000 & 0.17 & \pm 0.14 & 0.47 & \pm 0.10 & 0.47 & \pm 0.11 & 0.53 & $\pm 0.11^{b}$ & - & & 0.28 \\
\hline \multicolumn{13}{|l|}{ Fish, g/d } \\
\hline & 1992 & 0.16 & \pm 0.07 & 0.17 & \pm 0.13 & 0.28 & \pm 0.12 & 0.13 & \pm 0.16 & - & & \\
\hline & 2000 & 0.30 & \pm 0.12 & 0.54 & \pm 0.11 & 0.54 & \pm 0.1 & 0.23 & \pm 0.14 & - & & 0.43 \\
\hline
\end{tabular}

\footnotetext{
${ }^{1}$ All values are mean \pm SE. Results derived form a linear mixed model adjusted for age, baseline total energy intake (kcal), physical activity level $(<30,30-60,60-90,>90 \mathrm{~min} / \mathrm{d})$, smoking status (never, former and current smoker), alcohol intake (g/d), level of education (low, medium, high), vegetable consumption (g/d), fruit consumption (g/d), consumption of grains $(\mathrm{g} / \mathrm{d}$ ) and dieting habits (yes or no to being on a diet in the past $5 \mathrm{y}$ ).

${ }^{2}$ Range of total fresh meat consumption (g/d): Q1, $\leq 63 ; \mathrm{Q} 2,63-84 ; \mathrm{Q} 3,84-101 ; \mathrm{Q} 4,101-123 ; \mathrm{Q} 5,123-280$.

${ }^{3}$ Test for overall difference in BMI increase over time between groups of meat intake.

${ }^{4}$ Including beef, pork, minced meat, liver and other meat.

${ }^{5}$ Including beef and pork.

${ }^{b}$ Significantly higher BMI increase than in the lowest quintile of intake, as derived from the quintile-by-time interaction $(P<0.05)$.
}

When retrospectively examining the association between meat consumption reported at baseline and weight change from age 20 (reported at baseline) until baseline (age 55-69 y), we observed a positive linear trend across all quintiles of total meat consumption in both men and women $(P$-trend $<0.001)$ (Table 5$)$. Similar associations were observed for nearly all subtypes of meat, although consumption of processed meat was not associated with this long term weight change in men $(P$-trend $=0.73)$. 
Table 5: Change in BMI $\left(\mathrm{kg} / \mathrm{m}^{2}\right)$ from age 20 y to baseline and corresponding $95 \% \mathrm{Cl}$ according to quintiles/ categories of intake of fresh meat, types of fresh meat, processed meat and fish, measured at baseline in the Netherlands Cohort Study.

\begin{tabular}{|c|c|c|c|c|}
\hline \multirow[b]{2}{*}{ Food item } & \multicolumn{2}{|l|}{$\begin{array}{l}\text { Men } \\
\mathrm{N}=1598\end{array}$} & \multicolumn{2}{|l|}{$\begin{array}{l}\text { Women } \\
N=1736\end{array}$} \\
\hline & Change in $\mathrm{BMI}$ & $(95 \% \mathrm{Cl})^{1}$ & Change in BMI & $(95 \% \mathrm{Cl})^{1}$ \\
\hline \multicolumn{5}{|c|}{ Total fresh meat, $\mathrm{g} / \mathrm{d}^{2}$} \\
\hline $1^{3}$ & 2.82 & $(2.62-3.02)$ & 2.75 & $(2.50-3.00)$ \\
\hline 2 & 3.10 & $(2.97-3.23)$ & 3.30 & $(3.13-3.46)$ \\
\hline 3 & 3.24 & (3.13-3.36) & 3.60 & $(3.46-3.75)$ \\
\hline 4 & 3.40 & $(3.27-3.52)$ & 3.88 & $(3.72-4.04)$ \\
\hline 5 & 3.79 & $(3.57-4.01)$ & 4.55 & $(3.29-4.82)$ \\
\hline$P$-trend & $<0.001$ & & $<0.001$ & \\
\hline \multicolumn{5}{|c|}{$\begin{array}{l}\text { Fresh red meat, } \\
\mathrm{g} / \mathrm{d}^{4}\end{array}$} \\
\hline $1^{3}$ & 2.83 & $(2.63-3.02)$ & 2.86 & $(2.61-3.10)$ \\
\hline 2 & 3.09 & $(2.95-3.22)$ & 3.31 & $(3.14-3.47)$ \\
\hline 3 & 3.24 & $(3.12-3.35)$ & 3.58 & $(3.45-2.73)$ \\
\hline 4 & 3.40 & $(3.28-3.53)$ & 3.84 & $(3.69-4.00)$ \\
\hline 5 & 3.78 & $(3.57-4.01)$ & 4.47 & $(3.21-4.74)$ \\
\hline$P$-trend & $<0.001$ & & $<0.001$ & \\
\hline \multicolumn{5}{|l|}{ Beef, g/d } \\
\hline $1^{3}$ & 3.11 & $(2.94-3.28)$ & 3.44 & $(3.23-3.66)$ \\
\hline 2 & 3.18 & $(3.03-3.32)$ & 3.51 & $(3.33-3.68)$ \\
\hline 3 & 3.24 & (3.12-3.36) & 3.57 & (3.42-3.72) \\
\hline 4 & 3.32 & (3.19-3.44) & 3.67 & (3.51-3.82) \\
\hline 5 & 3.52 & $(3.30-3.74)$ & 3.88 & $(3.60-4.15)$ \\
\hline$P$-trend & 0.07 & & 0.07 & \\
\hline \multicolumn{5}{|l|}{ Pork, g/d } \\
\hline $1^{3}$ & 3.08 & $(2.90-3.27)$ & 3.13 & $(2.90-3.36)$ \\
\hline 2 & 3.17 & (3.03-3.31) & 3.35 & (3.17-3.52) \\
\hline 3 & 3.24 & $(3.13-3.37)$ & 3.55 & $(3.40-3.70)$ \\
\hline 4 & 3.32 & $(3.20-3.45)$ & 3.77 & $(3.62-3.93)$ \\
\hline 5 & 3.53 & (3.30-3.75) & 4.29 & (4.01-4.56) \\
\hline$P$-trend & 0.06 & & $<0.001$ & \\
\hline \multicolumn{5}{|c|}{ Minced meat, $g / d^{5}$} \\
\hline $1^{3}$ & 3.05 & $(2.88-3.22)$ & 4.45 & $(3.24-3.67)$ \\
\hline 2 & 3.14 & $(3.00-3.28)$ & 3.52 & $(3.35-3.69)$ \\
\hline 3 & 3.23 & $(3.11-3.35)$ & 3.58 & $(3.43-3.73)$ \\
\hline 4 & 3.33 & (3.21-3.46) & 3.67 & (3.51-3.82) \\
\hline 5 & 3.60 & $(3.39-3.81)$ & 3.87 & $(3.59-4.16)$ \\
\hline$P$-trend & 0.03 & & 0.01 & \\
\hline \multicolumn{5}{|c|}{ Chicken, $\mathrm{g} / \mathrm{d}^{6}$} \\
\hline $1^{3}$ & 3.23 & $(3.07-3.40)$ & 3.40 & $(3.20-3.60)$ \\
\hline 2 & 3.25 & (3.11-3.39) & 3.49 & $(3.32-3.66)$ \\
\hline 3 & 3.28 & (3.16-3.39) & 3.62 & (3.47-3.77) \\
\hline 4 & 3.33 & (3.14-3.51) & 3.91 & (3.68-4.15) \\
\hline$P$-trend & 0.06 & & 0.01 & \\
\hline \multicolumn{5}{|c|}{$\begin{array}{l}\text { Processed meat, } \\
\mathrm{g} / \mathrm{d}\end{array}$} \\
\hline $1^{3}$ & 3.15 & $(2.99-3.33)$ & 3.41 & $(3.21-3.61)$ \\
\hline 2 & 3.20 & (3.05-3.34) & 3.47 & (3.29-3.65) \\
\hline 3 & 3.24 & $(3.12-3.36)$ & 3.55 & $(3.39-3.70)$ \\
\hline 4 & 3.29 & $(3.18-3.42)$ & 3.67 & $(3.62-3.82)$ \\
\hline 5 & 3.47 & (3.24-3.70) & 3.98 & (3.70-4.27) \\
\hline$P$-trend & 0.73 & & $<0.001$ & \\
\hline
\end{tabular}




\begin{tabular}{clllll}
\hline & \multicolumn{2}{l}{$\begin{array}{l}\text { Men } \\
\mathrm{N}=1598\end{array}$} & & \multicolumn{2}{l}{$\begin{array}{l}\text { Women } \\
\mathrm{N}=1736\end{array}$} \\
\cline { 2 - 3 } \cline { 5 - 5 } Food item & Change in BMI & $(95 \% \mathrm{Cl})^{1}$ & & \\
\hline Fish, $\mathrm{g} / \mathrm{d}^{6}$ & & & & \\
$1^{3}$ & 3.21 & $(3.05-3.37)$ & 3.40 & $(3.21-3.60)$ \\
2 & 3.24 & $(3.10-3.37)$ & 3.50 & $(3.33-3.66)$ \\
3 & 3.28 & $(3.16-3.40)$ & 3.68 & $(3.53-3.84)$ \\
4 & 3.38 & $(3.17-3.59)$ & 4.02 & $(3.73-4.30)$ \\
$P$-trend & 0.02 & & 0.01 & \\
\hline \hline
\end{tabular}

\footnotetext{
${ }^{1}$ All values are mean $\pm 95 \% \mathrm{Cl}$. Results derived from a linear regression model adjusted for mean values of age, baseline total energy intake (kcal), alcohol intake $(\mathrm{g} / \mathrm{d})$, vegetable consumption $(\mathrm{g} / \mathrm{d})$, fruit consumption $(\mathrm{g} / \mathrm{d})$, consumption of grains $(\mathrm{g} / \mathrm{d})$, physical activity level, smoking status, level of education, dieting habits in the past $5 \mathrm{y}$ and $\mathrm{BMI}$ at age $20 \mathrm{y}$.

${ }^{2}$ Including all types of meat (except processed meat) and poultry.

${ }^{3}$ Reference category.

${ }^{4}$ Including beef, pork, minced meat, liver and other meat.

${ }^{5}$ Including beef and pork.

${ }^{6}$ Categorical cut points are for chicken: 0, 0-13.2, $13.2-22.8, \geq 22.8 \mathrm{~g} /$ day; fish: 0, 0-10, 10-20, $\geq 20 \mathrm{~g} /$ day.
}

\section{DISCUSSION}

This large scale observational study among 55-69 y old men and women showed no evidence of a prospective association between total meat consumption and subsequent changes in BMI over 14 y of follow-up.

Although the global rates of overweight and obesity are steadily increasing, we observed only a small overall increase in BMI during the $14 \mathrm{y}$ of follow-up. This is consistent with previous studies showing that men and women predominantly gain weight until middle age $[32,33]$. Furthermore, we did not find any evidence for an association between total (fresh) meat consumption and subsequent change in BMI in this population. This would suggest that the largest weight modifying effect of meat intake, if present, might have taken place earlier in adulthood. However, our age stratified (54-59, 60-64, 65-70 y) analyses were not indicative of a clear interaction-effect with age. Recent results from the EPIC-PANACEA study on the association between various meat products and 5 year weight change including subjects from a wider age range (25-70 y) showed the strongest association between weight and red meat intake in subjects aged $<35$ y or $>65$ y and with poultry consumption in subjects aged $>45$ y [21]. The few other observational studies on the association between total meat consumption and prospective weight gain report an overall tendency for a positive association [14-17, 21]. Two studies investigated the possible role of vegetarianism in relation to weight change but observed only small [12] or non-significant mixed results [13]. Yet, the length of follow-up (2-10 y) in these studies was relatively short and the quality of meat consumption assessment differed considerable between these studies, varying from comprehensive food frequency questionnaires $[14,15,21]$ to foodcategory based assessments $[16,17]$.

Our study is the first to further distinguish between the different components of red meat consumption and reports an inverse association between beef consumption and prospective changes in BMI in men. However, this beneficial effect on body weight was only present in subjects with very high intakes and attenuates below a consumption level of $40 \mathrm{~g} /$ day, thereby likely reflecting a dietary pattern in the extremes. Although beef consumption was positively correlated with a dietary pattern high in cooked leafy 
vegetables, cabbages, legumes and carrots $(r=0.20, P<0.001)$ and inversely correlated with a pattern high in pork, processed meat and potatoes ( $r=-0.16, P<0.001)$, adjustment for these patterns did not change the results noticeably (results not shown). In contrast, in women, the highest quintile of pork consumption was associated with a $0.60 \mathrm{~kg} / \mathrm{m}^{2}$ higher increase in BMI compared to the lowest intake, after 14 y of follow-up. The differences in nutritional content between beef and pork (e.g. the fat/protein ratio) may have resulted in these different weight inducing effects. Previous longitudinal studies examining changes in body weight [21] or waist circumference $[19,34]$ in relation to red meat consumption in general, showed contrasting results, but none of the reports specified the relative intakes of the individual components of red meat.

Our data suggest that men and women with the highest chicken consumption $(>22.8 \mathrm{~g} / \mathrm{d})$ had a 0.19 and $0.53 \mathrm{~kg} / \mathrm{m}^{2}$ higher increase in BMI after $14 \mathrm{y}$ of follow-up, respectively, compared to those who consumed no chicken at all. This is in line with recent results from a Danish study showing that high consumption of chicken was associated with greater weight gain at the waist after $5 \mathrm{y}$ of follow-up in women, but not in men [19]. The EPICPANACEA study recently reported a strong positive association between prospective weight change and chicken consumption but this was mainly driven by subjects with previous illness or weight loss attempts that resulted in changes in diet [21]. However, when we adjusted our analyses for special dieting habits (on doctor's advice) in the 5 y preceding the baseline questionnaire or excluded subjects who developed cancer during follow-up, the results did not change appreciably.

The discrepancy between our cross-sectional and prospective results raises the question during which age period the differences in BMI across the meat consumption spectrum develop. This prompted us to retrospective examine the association between meat consumption at baseline and change in body weight between age 20 and baseline (55-69 y). It should however be noted that we 1) only have baseline data on meat intake and 2) use long term recall of early adulthood weight. Although earlier studies indicate that the remote recall of weight by elderly subjects is overall accurate and independent of current weight status $[35,36]$, and that, in addition, there is no reason to assume differential recall bias with respect to meat consumption at baseline, we cannot empirically test these assumptions at present. However, in case these assumptions do hold our results suggest that meat consumption, or factors directly related to meat consumption, are positively associated with weight change over the first $40 \mathrm{y}$ of adult life. Studies specifically designed to examine long term prospective weight change, that combine multiple dietary- and anthropometric assessments are needed to verify these results.

We must be careful when drawing strong causal inferences from both our prospective and retrospective findings given that $\mathrm{BMI}$ is influenced by multiple connected dietary and nondietary factors of which meat consumption is only one. However, our large scale study does provide relevant clues for further research and public health practice as regards to identifying modifiable dietary determinants of obesity. The dietary pattern of our subcohort and the types of meat consumed are likely population- and time-specific and consumption of subtypes of meat are likely to be less stable over time than total meat intake. We cannot completely exclude the possibility that individuals with the lowest total meat intake may have had an overall healthier lifestyle than did those with the highest 
intake, resulting in less of a BMI increase over time. Nevertheless, when we attempted to control for further potential confounding in our model by accounting for intake of vegetables, fruit, grain content of the diet and dieting habits our observations did not change appreciably. We made use of a food frequency questionnaire to determine meat consumption, which was found to correlate well with a measurement using dietary records [37] but is nonetheless susceptible for measurement error [38]. We were not able to account for changes in physical activity or dietary intake which is likely to occur with ageing. However, the validity of the FFQ has been tested and shown to be representative for dietary habits over a period of at least 5 y [39]. Self-reported height and weight measurements recorded in the questionnaire were not validated within the NLCS, but previous studies provide sufficient evidence to presume that these are reliable and valid tools to ascertain body weight and height in large scale prospective studies [40-42]. Nevertheless, the prevalence of overweight might have been underestimated especially since body height is known to decrease and body composition is likely to change at older age [43]. Because we only have BMI measurements we cannot distinguish between changes in lean tissue or fat mass; a shortcoming of many observational studies which is currently debated in literature $[44,45]$. However, several studies reported similar findings when comparing changes in BMI to changes in waist circumference, a proxy of abdominal adiposity. Generalization of our results to younger populations may be hampered since ageing is accompanied by specific changes in body composition and metabolic function that could have affected our findings. Body weight is likely to fluctuate over time resulting in successive periods of weight loss and gain [46, 47]. Our long follow-up including three consecutive self-reports reduces the influence of such weight cycling on the study results. Because of the older age group of our population at baseline many subcohort members only had one or two prospective measurements available for analysis. Although it is likely that those who remained alive until the last follow-up point tend to be healthier than the entire subcohort, there was no indication that survivorship bias distorted our results.

Our data suggest that meat consumption, or factors directly related to meat intake, is not strongly associated with weight change during the 14-year prospective follow-up in this elderly population. However, more observational and intervention research is needed to 1 ) verify our prospective and retrospective observations and 2) to further elucidate the role of (subtypes of) meat in weight management and its implications for public health practice.

\section{REFERENCES}

1. World Health Organisation. Obesity and overweight. Factsheet no 311. http://www.who.int/mediacentre/factsheets/fs311/en/. 2006 31-05-2010].

2. Flegal, K.M., et al., Prevalence and trends in obesity among US adults, 1999-2008. Jama. 303(3): p. 235-41.

3. Spencer, E.A., et al., Diet and body mass index in 38000 EPIC-Oxford meat-eaters, fish-eaters, vegetarians and vegans. Int J Obes Relat Metab Disord, 2003. 27(6): p. 728-34.

4. Appleby, P.N., et al., Low body mass index in non-meat eaters: the possible roles of animal fat, dietary fibre and alcohol. Int J Obes Relat Metab Disord, 1998. 22(5): p. 454-60.

5. Fraser, G.E., Associations between diet and cancer, ischemic heart disease, and all-cause mortality in non-Hispanic white California Seventh-day Adventists. Am J Clin Nutr, 1999. 70(3 Suppl): p. 532S-538S.

6. Key, T.J., et al., Mortality in vegetarians and non-vegetarians: a collaborative analysis of 8300 deaths among 76,000 men and women in five prospective studies. Public Health Nutr, 1998. 1(1): p. 33-41. 
7. Newby, P.K., K.L. Tucker, and A. Wolk, Risk of overweight and obesity among semivegetarian, lactovegetarian, and vegan women. Am J Clin Nutr, 2005. 81(6): p. 1267-74.

8. Togo, P., et al., Food intake patterns and body mass index in observational studies. Int J Obes Relat Metab Disord, 2001. 25(12): p. 1741-51.

9. Nicklas, T.A., et al., Impact of meat consumption on nutritional quality and cardiovascular risk factors in young adults: the Bogalusa Heart Study. J Am Diet Assoc, 1995. 95(8): p. 887-92.

10. Vang, A., et al., Meats, processed meats, obesity, weight gain and occurrence of diabetes among adults: findings from Adventist Health Studies. Ann Nutr Metab, 2008. 52(2): p. 96-104.

11. Wang, Y. and M.A. Beydoun, Meat consumption is associated with obesity and central obesity among US adults. Int J Obes (Lond), 2009. 33(6): p. 621-8.

12. Rosell, M., et al., Weight gain over 5 years in 21,966 meat-eating, fish-eating, vegetarian, and vegan men and women in EPIC-Oxford. Int J Obes (Lond), 2006. 30(9): p. 1389-96.

13. Fraser, G.E., Diet, Life Expectancy, and Chronic Disease. Studies of Seventh-day Adventists and Other Vegetarians. 2003, New York: Oxford University Press.

14. Schulz, M., et al., Food groups as predictors for short-term weight changes in men and women of the EPIC-Potsdam cohort. J Nutr, 2002. 132(6): p. 1335-40.

15. Kahn, H.S., L.M. Tatham, and C.W. Heath, Jr., Contrasting factors associated with abdominal and peripheral weight gain among adult women. Int J Obes Relat Metab Disord, 1997. 21(10): p. 903-11.

16. Kahn, H.S., et al., Stable behaviors associated with adults' 10 -year change in body mass index and likelihood of gain at the waist. Am J Public Health, 1997. 87(5): p. 747-54.

17. Halkjaer, J., et al., Food and drinking patterns as predictors of 6-year BMI-adjusted changes in waist circumference. Br J Nutr, 2004. 92(4): p. 735-48.

18. Parker, D.R., et al., Dietary factors in relation to weight change among men and women from two southeastern New England communities. Int J Obes Relat Metab Disord, 1997. 21(2): p. 103-9.

19. Halkjaer, J., et al., Dietary predictors of 5-year changes in waist circumference. J Am Diet Assoc, 2009. 109(8): p. 1356-66.

20. Drapeau, V., et al., Modifications in food-group consumption are related to long-term body-weight changes. Am J Clin Nutr, 2004. 80(1): p. 29-37.

21. Vergnaud, A.C., et al., Meat consumption and prospective weight change in participants of the EPICPANACEA study. Am J Clin Nutr, 2010. 92 (2): p. 398-407.

22. Westerterp-Plantenga, M.S., et al., Dietary protein, weight loss, and weight maintenance. Annu Rev Nutr, 2009. 29: p. 21-41.

23. Nordmann, A.J., et al., Effects of low-carbohydrate vs low-fat diets on weight loss and cardiovascular risk factors: a meta-analysis of randomized controlled trials. Arch Intern Med, 2006. 166(3): p. 285-93.

24. Atkins, R.C., Atkins for Life: The Complete Controlled Carb Program for Permanent Weight Loss and Good Health. 2004, New York: NY: St Martins Press.

25. Gardner, C.D., et al., Comparison of the Atkins, Zone, Ornish, and LEARN diets for change in weight and related risk factors among overweight premenopausal women: the A TO Z Weight Loss Study: a randomized trial. Jama, 2007. 297(9): p. 969-77.

26. Truby, $\mathrm{H}_{\text {., }}$ et al., Randomised controlled trial of four commercial weight loss programmes in the UK: initial findings from the BBC “diet trials". Bmj, 2006. 332(7553): p. 1309-14.

27. Astrup, A., T. Meinert Larsen, and A. Harper, Atkins and other low-carbohydrate diets: hoax or an effective tool for weight loss? Lancet, 2004. 364(9437): p. 897-9.

28. Dansinger, M.L., et al., Comparison of the Atkins, Ornish, Weight Watchers, and Zone diets for weight loss and heart disease risk reduction: a randomized trial. Jama, 2005. 293(1): p. 43-53.

29. van den Brandt, P.A., et al., A large-scale prospective cohort study on diet and cancer in The Netherlands. J Clin Epidemiol, 1990. 43(3): p. 285-95.

30. Phillips, S.M., et al., Dairy food consumption and body weight and fatness studied longitudinally over the adolescent period. Int J Obes Relat Metab Disord, 2003. 27(9): p. 1106-13.

31. Finucane, M.M., J.H. Samet, and N.J. Horton, Translational methods in biostatistics: linear mixed effect regression models of alcohol consumption and HIV disease progression over time. Epidemiol Perspect Innov, 2007. 4: p. 8.

32. Guo, S.S., et al., Aging, body composition, and lifestyle: the Fels Longitudinal Study. Am J Clin Nutr, 1999. 70(3): p. 405-11. 
33. Sheehan, T.J., et al., Rates of weight change for black and white Americans over a twenty year period. Int J Obes Relat Metab Disord, 2003. 27(4): p. 498-504.

34. Wagemakers, J.J., et al., Consumption of red or processed meat does not predict risk factors for coronary heart disease; results from a cohort of British adults in 1989 and 1999. Eur J Clin Nutr, 2009. 63(3): p. 303-11.

35. Must, A., W.C. Willett, and W.H. Dietz, Remote recall of childhood height, weight, and body build by elderly subjects. Am J Epidemiol, 1993. 138(1): p. 56-64.

36. Norgan, N.G. and N. Cameron, The accuracy of body weight and height recall in middle-aged men. Int J Obes Relat Metab Disord, 2000. 24(12): p. 1695-8.

37. Goldbohm, R.A., et al., Validation of a dietary questionnaire used in a large-scale prospective cohort study on diet and cancer. Eur J Clin Nutr, 1994. 48(4): p. 253-65.

38. Kipnis, V., et al., Structure of dietary measurement error: results of the OPEN biomarker study. Am J Epidemiol, 2003. 158(1): p. 14-21; discussion 22-6.

39. Goldbohm, R.A., et al., Reproducibility of a food frequency questionnaire and stability of dietary habits determined from five annually repeated measurements. Eur J Clin Nutr, 1995. 49(6): p. 420-9.

40. Wada, K., et al., Validity of self-reported height and weight in a Japanese workplace population. Int J Obes (Lond), 2005. 29(9): p. 1093-9.

41. Spencer, E.A., et al., Validity of self-reported height and weight in 4808 EPIC-Oxford participants. Public Health Nutr, 2002. 5(4): p. 561-5.

42. Nyholm, M., et al., The validity of obesity based on self-reported weight and height: Implications for population studies. Obesity (Silver Spring), 2007. 15(1): p. 197-208.

43. Dey, D.K., et al., Height and body weight in the elderly. I. A 25-year longitudinal study of a population aged 70 to 95 years. Eur J Clin Nutr, 1999. 53(12): p. 905-14.

44. Astrup, A., et al., Meat intake's influence on body fatness cannot be assessed without measurement of body fat. Am J Clin Nutr, 2010. 92(5): p. 1274-5; author reply 1275-6.

45. Vergnaud, A.C., et al., Reply to A Astrup et al. Am J Clin Nutr, 2010. 92 p. 1275-1276.

46. Kramer, F.M., et al., Long-term follow-up of behavioral treatment for obesity: patterns of weight regain among men and women. Int J Obes, 1989. 13(2): p. 123-36.

47. Lahti-Koski, M., et al., Prevalence of weight cycling and its relation to health indicators in Finland. Obes Res, 2005. 13(2): p. 333-41.

Data collection

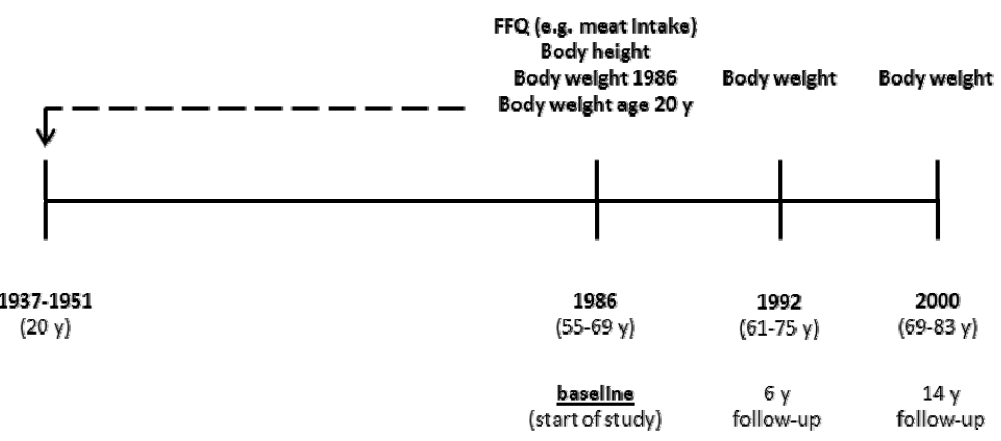

Supplemental Figure 1:

Overview of the study design for the BMI change analyses in the subcohort of the Netherlands Cohort Study 



\title{
CHAPTER 3
}

Serum concentrations of vitamin B12 and folate in British male vegans, vegetarians and omnivores: results from a cross-sectional analysis of the EPIC-Oxford cohort study

\author{
Anne M.J. Gilsing \\ Francesca L. Crowe \\ Zouë Lloyd-Wright \\ Thomas A.B. Sanders \\ Paul N. Appleby \\ Naomi E. Allen \\ and Timothy J. Key
}

European Journal of Clinical Nutrition 2010;64(9):933-9 


\section{ABSTRACT}

Background: Vegans and to a lesser extent vegetarians have low average circulating concentrations of vitamin B12; however, the relation between factors such as age or time on these diets and vitamin B12 concentrations is not clear. The objectives were to investigate differences in serum vitamin B12 and folate concentrations between omnivores, vegetarians and vegans and to ascertain whether vitamin B12 concentrations differed by age and time on the diet.

Methods: A cross-sectional analysis involving 689 men (226 omnivores, 231 vegetarians and 232 vegans) from the European Prospective Investigation into Cancer and Nutrition Oxford cohort.

Results: Mean serum vitamin B12 was highest among omnivores (281, 95\% Cl: 271-291 $\mathrm{pmol} / \mathrm{l})$, intermediate in vegetarians $(182,95 \% \mathrm{Cl}: 175-189 \mathrm{pmol} / \mathrm{l})$, and lowest in vegans (122, 95\% Cl: $117-127 \mathrm{pmol} / \mathrm{l})$. Fifty-two percent of vegans, $7 \%$ of vegetarians and one omnivore were classified as vitamin B12 deficient (defined as serum vitamin B12<118 $\mathrm{pmol} / \mathrm{I}$ ). Among non-supplement users, there was no significant association between age or duration of adherence to a vegetarian or a vegan diet and vitamin B12. In contrast, folate concentrations were highest among vegans, intermediate in vegetarians, and lowest in omnivores, but only two men (both omnivores) were categorized as folate deficient (defined as serum folate $<6.3 \mathrm{nmol} / \mathrm{l}$ ).

Conclusion: Vegans have lower vitamin B12 concentrations, but higher folate concentrations, than vegetarians and omnivores. Nearly half of the vegans were categorized as vitamin B12 deficient and would be expected to have a higher risk of developing clinical symptoms related to vitamin B12 deficiency. 


\section{INTRODUCTION}

Although individuals who consume a vegetarian and vegan diets may have benefits that include a lower risk of cardiovascular disease [1, 2], there may also be a greater risk of developing deficiencies, because of the exclusion of meat and fish from the diet in vegetarians, and of all animal products in vegans. Vitamin B12 is naturally present only in foods of animal origin, and vegans who do not consume sufficient quantities of foods fortified with vitamin B12 such as some breakfast cereals, plant-based milks, soy products and yeast extract, or regularly take a vitamin B12 supplement, will have an increased risk of developing vitamin B12 deficiency.

Results from previous studies have shown that vegans have lower average serum concentrations of vitamin B12 in comparison to omnivores and vegetarians [3-5], with evidence from some [4-8], but not all $[3,9,10]$ studies suggesting that serum vitamin B12 concentrations are also lower in vegetarians compared to omnivores. Several reports also indicate that a considerable proportion vegans $[10,11]$ and vegetarians $[5,12]$ have circulating concentrations of vitamin B12 indicative of depleted stores (serum vitamin B12 $<150 \mathrm{pmol} / \mathrm{l}$ ). Previous research in populations consuming a mixed diet suggests that serum vitamin B12 decreases with age [13]; however, few studies have assessed this in older vegetarians and vegans. Moreover, it is not clear to what extent factors such the length of adherence to a vegan or vegetarian diet is associated with serum concentrations of vitamin B12.

The objective of the present study was to report the serum vitamin B12 and folate concentrations and dietary intake of these micronutrients in British men consuming an omnivorous, vegetarian or vegan diet and to assess the associations of age and duration of adherence to a vegetarian or vegan diet with serum concentrations of vitamin B12.

\section{METHODS}

\section{Study population}

The European Prospective Investigation into Cancer and Nutrition (EPIC)-Oxford cohort study recruited more than 65000 participants between 1993 and 1999. Full details of the recruitment methods and the study participants have been described elsewhere [14]. The protocol for the EPIC-Oxford study was approved by the Royal College of General Practitioners' Clinical Research Ethics Committee, the Central Oxford Research Ethics Committee and local research ethics committees, and all participants gave written informed consent.

This study population sample consists of 689 men who provided a blood sample at recruitment to the EPIC-Oxford cohort between 1994 and 1997 and who had no history of cancer. To maximize the heterogeneity of dietary exposure, approximately equal numbers of men with different dietary habits were selected with no matching criteria: omnivores who reported three or more servings of meat per week; lacto-ovo vegetarians who reported consuming soya-milk and/or soya products, and vegans. There were 226 omnivores, 231 vegetarians, and 232 vegans included in these analyses. The ethnicity of $96 \%$ of the study sample was white and the other $4 \%$ were Bangladeshi or Chinese. 
Omnivores were defined as people reporting meat consumption. Vegetarians were defined as people who reported consuming dairy products (including milk, cheese, butter, and yogurt) and eggs (including eggs in cakes and other baked foods) but no meat or fish. The majority of vegetarians were lacto-ovo vegetarians (88\%), consuming both dairy products and eggs, with smaller proportions avoiding eggs $(8 \%)$ or dairy products $(4 \%)$. Vegans were defined on the basis that they did not to consume any foods of animal origin (meat, fish, dairy products or eggs). The duration of adherence to a vegetarian and vegan diet was calculated as the age at recruitment minus the age at which the respondent last ate meat or fish, or any of meat, fish, dairy products or eggs, respectively.

\section{Assessment of dietary and lifestyle variables}

All participants completed a validated semi-quantitative 130-item food frequency questionnaire (FFQ) at baseline [15, 16]. Regular use of a vitamins supplement over the past 12 months was assessed in the FFQ and participants were categorized on the basis of whether they regularly used a vitamin B12 supplement or folate supplement. The daily intakes of vitamin B12 and folate obtained from supplements were also calculated. The 12 men who did not provide any information on the use of supplements were categorized as non-users for each of these variables. Participants self-reported their height and weight, and Quetelet's body mass index (BMl; weight $(\mathrm{kg}) /$ height $\left(\mathrm{m}^{2}\right)$ ) was calculated. Participants were further characterized by their smoking status ("never", "former", "current") and level of education ("some secondary school"; "higher secondary school", "university degree or equivalent").

\section{Laboratory methods}

Following recruitment, blood was collected at local general practice surgeries into $10-\mathrm{mL}$ Safety-Monovettes (Sarstedt, Nümbrecht, Germany). The samples were sent by post in sealed containers at ambient temperature to the EPIC laboratories in Norfolk where they were centrifuged and aliquots were stored in liquid nitrogen $\left(-196^{\circ} \mathrm{C}\right)$ until analysis. Concentrations of vitamin B12 and folate in serum were measured with the use of a Quantaphase II B12/Folate Radioassay (Bio-Rad, CA) at the Department of Clinical Biochemistry, King's College Hospital, London. The inter-batch coefficients of variation (CV) were $7.9 \%$ for vitamin B12 and $7.7 \%$ for folate. Serum concentrations of vitamin B12 less than $118 \mathrm{pmol} / \mathrm{L}$ and serum folate concentrations less than $6.3 \mathrm{nmol} / \mathrm{L}$ were used as cutpoints to categorize deficiency as used in United Kingdom National Diet and Nutrition Survey [17].

\section{Follow-up data}

In 2001, the vegan men who had a serum vitamin B12 measurement were contacted and asked to provide another blood sample. Out of the 227 surviving vegan men contacted, another blood sample was collected from $65(29 \%)$ of them. Full-blood counts were made using a Sysmex cell counter (Sysmex, Milton Keynes, UK); serum vitamin B12, serum holotranscobalamin (holoTC), plasma methylmalonic acid (MMA), and plasma total homocysteine (tHcy) were measured as described in detail elsewhere [18].

\section{Statistical Analyses}

All analyses were performed using Stata Statistical Software (release 9; Stata-Corp LP, College Station, TX). Where necessary, dietary variables and serum vitamin B12 and folate 
concentrations were log-transformed to approximate a normal distribution. To assess the effect of duration of adherence to a vegetarian or vegan diet on serum vitamin B12 concentrations, vegetarians and vegans were divided into four categories $(\leq 5,6-10,11-15$, $\geq 16$ y) according to the time they had been on these diets. The geometric mean and $95 \% \mathrm{Cl}$ for serum B12 were calculated for nine diet-duration groups (omnivores, 4 groups of vegetarians and 4 groups of vegans) by using multiple linear regression. Categories of BMI $(<22.5,22.5-24.9, \geq 25 \mathrm{~kg} / \mathrm{m} 2)$, use of a vitamin B12 supplement, smoking status, level of education, number of days the blood sample spent in the post (1, 2 or $\geq 3$ days) and alcohol consumption (quartiles) were included as covariates, but only BMI, education, and supplement use were included in the final model. Similar analyses were performed to investigate the associations of age and education with serum vitamin B12 concentrations. $\mathrm{P}$ values for trend for the association between time of adherence to the diet and age with serum concentrations of vitamin B12 were assessed separately for each diet group by treating age and duration on the diet as continuous variables in the regression models. All tests were two-tailed and differences were regarded as statistically significant at $P<0.05$.

\section{RESULTS}

The mean age of the participants was $47 \mathrm{y}$; omnivores were on average $7 \mathrm{y}$ older than vegetarians and $10 \mathrm{y}$ older than vegans (Table 1 ).

Table 1 : Characteristics of the EPIC-Oxford men by diet group

\begin{tabular}{|c|c|c|c|c|}
\hline & $\begin{array}{l}\text { Omnivores } \\
\mathrm{n}=226\end{array}$ & $\begin{array}{l}\text { Vegetarians } \\
\mathrm{n}=231\end{array}$ & $\begin{array}{l}\text { Vegans } \\
\mathrm{n}=232\end{array}$ & $P^{1}$ \\
\hline Age (y) (mean (s.d.)) & $52.8(10.7)$ & $46.2(11.7)$ & $42.8(13.1)$ & $<0.001$ \\
\hline BMI $\left(\mathrm{kg} / \mathrm{m}^{2}\right)($ mean (s.d.)) & $26.1(3.7)$ & $23.4(3.0)$ & $22.7(3.1)$ & $<0.001$ \\
\hline \multicolumn{5}{|l|}{ Age category } \\
\hline $20-39$ & $15(7)$ & $75(32)$ & $104(45)$ & \multirow{4}{*}{$<0.001$} \\
\hline $40-49$ & $85(38)$ & $79(34)$ & $64(28)$ & \\
\hline $50-59$ & $55(24)$ & 40 (17) & $31(13)$ & \\
\hline $60-78$ & $71(31)$ & $37(16)$ & $33(14)$ & \\
\hline \multicolumn{5}{|l|}{ BMI category } \\
\hline$<22.5$ & $37(16)$ & $93(40)$ & $125(54)$ & \multirow{3}{*}{$<0.001$} \\
\hline $22.5-24 \cdot 9$ & $58(26)$ & $87(38)$ & $70(30)$ & \\
\hline$\geq 25 \cdot 0$ & $131(58)$ & $51(22)$ & $37(16)$ & \\
\hline \multicolumn{5}{|l|}{ Education } \\
\hline Some secondary school & $54(24)$ & $35(15)$ & $32(14)$ & \multirow{3}{*}{0.023} \\
\hline Higher secondary school & $71(31)$ & $69(30)$ & $82(35)$ & \\
\hline University degree or equivalent & $101(45)$ & $127(55)$ & $118(51)$ & \\
\hline \multicolumn{5}{|l|}{ Smoking } \\
\hline Never & $109(48)$ & $121(52)$ & $139(60)$ & \multirow{3}{*}{0.015} \\
\hline Former & $80(35)$ & $90(39)$ & $70(30)$ & \\
\hline Current & $37(16)$ & $20(9)$ & $23(10)$ & \\
\hline \multicolumn{5}{|l|}{ Vitamin $B_{12}$ supplement users } \\
\hline Yes & $10(4)$ & $45(19)$ & $43(19)$ & \multirow{2}{*}{$<0.001$} \\
\hline No & $216(96)$ & $186(81)$ & $189(81)$ & \\
\hline \multicolumn{5}{|l|}{ Time on vegetarian or vegan diet (y) } \\
\hline$\leq 5 y$ & - & $54(23)$ & $89(38)$ & \\
\hline $6-10$ y & - & $56(24)$ & $69(30)$ & \\
\hline $11-15$ y & - & 44 (19) & 44 (19) & \\
\hline$\geq 16 y$ & - & $74(32)$ & $29(13)$ & \\
\hline
\end{tabular}

Values are $\mathrm{n}(\%)$, except where indicated otherwise.

${ }^{1} P$-values derived from ANOVA or $\chi^{2}$ test. 
Vegans had the lowest mean BMI $(P<0.001)$ and a greater proportion of omnivores had an education below secondary level compared to vegetarians and vegans. The median time that vegetarian men had adhered to their diet was $11 \mathrm{y}$ and for vegan men it was $7 \mathrm{y}$. Among the omnivores, $4 \%$ regularly took a supplement containing vitamin B12 compared to $19 \%$ of both vegetarians and vegans.

The mean intake of dietary vitamin B12 was $8.8 \mu \mathrm{g}$ among omnivores, which was almost five times greater than the mean intake in vegetarians $(P<0.001)$ and 36 times higher than the mean intake in vegans $(P<0.001$, Table 2$)$. Only $3 \%$ of the vegan men not taking a vitamin B12 supplement reported a dietary intake of vitamin B12 above the UK RNI of 1.5 $\mu \mathrm{g} /$ day compared to $31 \%$ of the vegetarians and all omnivores. Among vitamin B12 supplement users, $89 \%$ of the vegetarians and $63 \%$ of vegans met the RNI for vitamin B12 intake. Vegans not taking a folate acid supplement had a significantly higher intake of folate than both vegetarians $(P=0.001)$ and omnivores $(P<0.001)$ and the mean intake of folate in the vegetarians was higher than that in the omnivores $(P=0.014)$. Dietary intake of folate was above the RNI ( $200 \mu \mathrm{g} / \mathrm{day})$ in $96 \%$ of the omnivores, $99 \%$ of the vegetarians and $98 \%$ of the vegans. All supplement users had an intake of folate (from diet and supplements) that was above the RNI.

Table 2: Estimated daily intake among omnivores, vegetarians and vegans

\begin{tabular}{|c|c|c|c|}
\hline & $\begin{array}{l}\text { Omnivores } \\
(\mathrm{n}=226)\end{array}$ & $\begin{array}{l}\text { Vegetarians } \\
(\mathrm{n}=231)\end{array}$ & $\begin{array}{l}\text { Vegans } \\
(n=232)\end{array}$ \\
\hline \multicolumn{4}{|c|}{ Vitamin B12 intake ( $\mu$ g/day) [mean $(95 \% \mathrm{Cl})]$} \\
\hline Non-supplement users & $8.76(7.93-9.68)^{1 a}$ & $1.92(1.72-2.13)^{b}$ & $0.24(0.21-0.26)^{\mathrm{c}}$ \\
\hline Supplement users ${ }^{2}$ & $11.06(6.21-19.67)^{a}$ & $3.39(2.58-4.45)^{b}$ & $3.17(2.40-4.19)^{b}$ \\
\hline \multicolumn{4}{|c|}{ Folate intake $(\mu \mathrm{g} /$ day) [mean $(95 \% \mathrm{Cl})]$} \\
\hline Non-supplement users & $342(329-357)^{a}$ & $369(353-386)^{b}$ & $420(402-438)^{c}$ \\
\hline Supplement users ${ }^{2}$ & $595(513-690)$ & $610(566-658)$ & $611(564-663)$ \\
\hline Total energy intake (MJ) & $10.6(2.7)$ & $9.2(2.5)$ & $8.5(2.6)$ \\
\hline Carbohydrate (\% total energy) & $43.6(5.9)$ & $51.5(6.4)$ & $53.4(7.8)$ \\
\hline Fat (\% total energy) & $34.0(5.3)$ & $31.0(5.7)$ & $29.9(7.4)$ \\
\hline Protein (\% total energy) & $16.5(2.7)$ & $13.2(2.0)$ & $12.7(1.9)$ \\
\hline
\end{tabular}

The mean serum vitamin B12 in vegans was $33 \%$ lower than in vegetarians and $57 \%$ lower than in omnivores, and was $35 \%$ lower in vegetarians compared to omnivores (Table 3 ). In all, $52 \%$ of vegans and $7 \%$ of vegetarians had vitamin B12 concentrations below the cutpoint for biochemical deficiency $(<118 \mathrm{pmol} / \mathrm{l})$. A further 21,17 and $1 \%$ of vegans, vegetarians and omnivores, respectively, had a serum vitamin B12 indicative of depletion (118 to $150 \mathrm{pmol} / \mathrm{l})$. There was no significant difference in mean serum concentration of vitamin B12 between men who reported taking a vitamin B12 supplement compared to non-users of supplements in any of the diet groups (results not shown).

The mean concentration of serum folate in vegans was $34 \%$ higher than in vegetarians and $88 \%$ higher than in omnivores (Table 3 ). Very few men $(<1 \%$ of omnivores) were 
categorized with biochemical folate deficiency $(<6.3 \mathrm{nmol} / \mathrm{l})$. Vegetarians who reported taking a folate supplement had significantly higher mean serum folate concentrations compared to non-supplement users $(P=0.015)$, but mean serum folate concentrations between supplement and non-supplement users did not differ for omnivores $(P=0.738)$ and vegans ( $P=0.072$, results not shown).

Table 3: Serum concentrations of vitamin $\mathrm{B}_{12}$ and folate according to diet group

\begin{tabular}{llll}
\hline & $\begin{array}{l}\text { Omnivores } \\
(\mathrm{n}=226)\end{array}$ & $\begin{array}{l}\text { Vegetarians } \\
(\mathrm{n}=231)\end{array}$ & $\begin{array}{l}\text { Vegans } \\
(\mathrm{n}=232)\end{array}$ \\
\hline $\begin{array}{l}\text { Serum Vitamin } \mathrm{B}_{12} \\
(\mathrm{pmol} / \mathrm{L})[\text { mean }(95 \% \mathrm{Cl})]\end{array}$ & $281(270-292)^{1 \mathrm{a}}$ & $182(175-189)^{\mathrm{b}}$ & $122(117-127)^{\mathrm{c}}$ \\
& & & \\
"Deficient" $<118 \mathrm{pmol} / \mathrm{L}$ & $1(0)$ & $16(7)$ & $121(52)$ \\
"Depleted" 118 to $149 \mathrm{pmol} / \mathrm{L}$ & $3(1)$ & $40(17)$ & $48(21)$ \\
"Sufficient" $\geq 150 \mathrm{pmol} / \mathrm{L}$ & $222(98)$ & $175(76)$ & $63(27)$ \\
& $20 \cdot 0(19 \cdot 1-21 \cdot 0)^{\mathrm{a}}$ & $28 \cdot 0(26 \cdot 7-29 \cdot 4)^{\mathrm{b}}$ & $37 \cdot 5(35 \cdot 8-39 \cdot 3)^{\mathrm{c}}$ \\
$\begin{array}{l}\text { Serum Folate } \\
\text { (nmol/L) [mean }(95 \% \mathrm{Cl})]\end{array}$ & & & \\
& $2(1)$ & 0 & 0 \\
"Deficient" $<6.3 \mathrm{nmol} / \mathrm{L}$ & $224(99)$ & $231(100)$ & $232(100)$ \\
"Sufficient" $\geq 6.3 \mathrm{nmol} / \mathrm{L}$ & & & \\
\hline \hline
\end{tabular}

Values are $\mathrm{n}(\%)$, except where indicated otherwise.

${ }^{1}$ Differences in the means between the diet groups were tested for by using ANOVA with Bonferroni's adjustment.

$a, b, c$ Different superscript letters indicate statistically significant differences, $P<0.001$.

Among participants not taking a supplement, dietary intake of vitamin B12 was significantly correlated with serum vitamin B12 concentrations (Spearman's $\rho=0.72, P<0.001$ ). The correlation between dietary folate intake and serum concentrations of folate was weaker but statistically significant $(\rho=0.12, P=0.002)$.

The adjusted mean concentrations of serum vitamin B12 by time of adherence to a vegetarian or vegan diet and by age for non-supplement users are shown in Figure 1 . There was no significant association between the length of adherence to a vegetarian or vegan diet and serum vitamin B12 concentrations. Neither was there a significant association between age or education and serum concentrations of vitamin B12 in any diet group (results for education not shown). All results were similar when restricted to nonusers of vitamin B12 supplements (results not shown).

Of the 65 vegan men with repeated measures of serum vitamin B12 taken approximately 6 years later, $34 \%$ were considered as biochemically vitamin B12 (serum vitamin B12 < 118 $\mathrm{pmol} / \mathrm{l}$ ) and $8 \%$ were categorized as depleted (118 to $150 \mathrm{pmol} / \mathrm{l})$ (Table 4). There was a strong positive association between serum vitamin B12 concentration and holotranscobalamin (holoTC) $(r=0.77, P<0.001)$ and an inverse association with methylmalonic acid and total homocysteine $(r=-0.74$ and $r=-0.73$, respectively; $P<0.0001$ for both). Among the 22 men who had serum vitamin B12 concentrations below $118 \mathrm{pmol} / \mathrm{l}$, $82 \%$ had concentrations of holoTC that would indicate vitamin B12 deficiency $(<35 \mathrm{pmol} / \mathrm{l})$, but only $32 \%$ had a combination of a methylmalonic acid greater than $0.75 \mu \mathrm{mol} / \mathrm{l}$ and total homocysteine greater than $15 \mu \mathrm{mol} / \mathrm{l}$ that would identify men who were likely to have a vitamin B12 deficiency. 


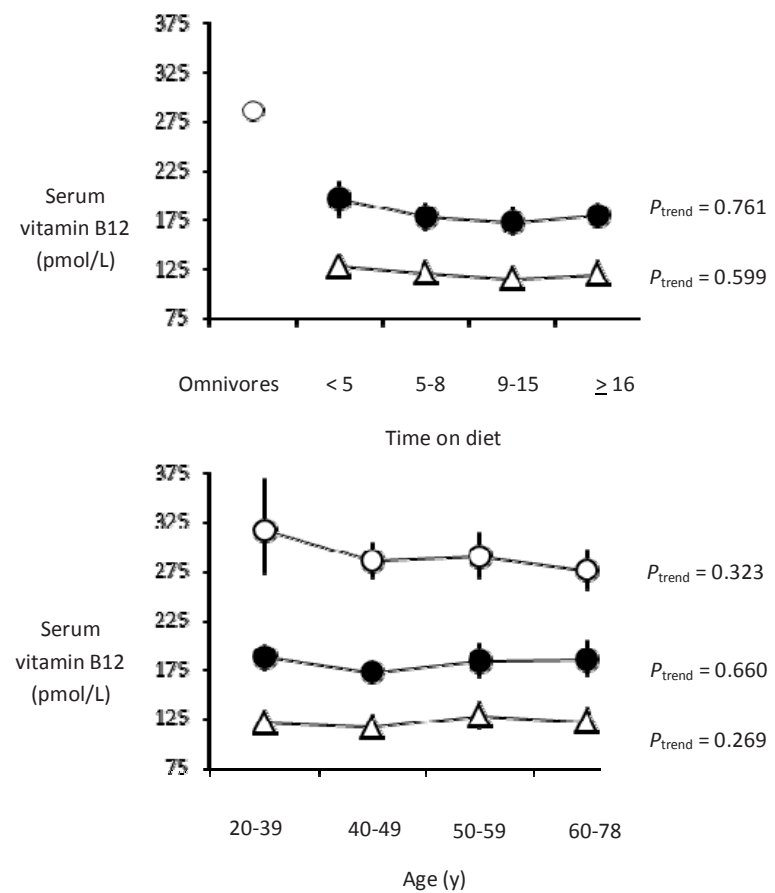

Figure 1. Mean serum concentrations of vitamin B12 in omnivores $(n=226)$, vegetarians $(n=231)$ and vegans $(n=$ 232 ) by duration of adherence to a vegetarian or a vegan diet (top panel) and age (bottom panel). The results in the figure show the geometric mean and $95 \% \mathrm{Cl}$ adjusted for level of education and use of a dietary supplement, by diet group ( $\circ$, omnivores; $\bullet$, vegetarians; and $\Delta$, vegans). $P$ values for trend by duration of adherence to a vegetarian or vegan diet and age within each diet group were obtained by treating the number of years since becoming vegetarian or vegan and age as continuous variables in the regression analyses, respectively.

Table 4: Geometric mean $(95 \% \mathrm{Cl})$ concentrations of selected biochemical measurements according to categories of serum vitamin $\mathrm{B}_{12}$ concentrations in 65 vegan men with follow-up data.

\begin{tabular}{|c|c|c|c|c|}
\hline & $\begin{array}{l}<118 \mathrm{pmol} / \mathrm{L} \\
\mathrm{n}=22\end{array}$ & $\begin{array}{l}118-149 \mathrm{pmol} / \mathrm{L} \\
\mathrm{n}=5\end{array}$ & $\begin{array}{l}\geq 150 \mathrm{pmol} / \mathrm{L} \\
\mathrm{n}=38\end{array}$ & $P^{1}$ \\
\hline Serum vitamin B12 (pmol/L) & $51(40-64)$ & $131(81-213)$ & $302(253-360)$ & \\
\hline HoloTCII (pmol/L) & $26(20-33)$ & $29(20-41)$ & $77(65-92)$ & $<0.001$ \\
\hline HoloTCII < 35 pmol/L (\%) & 82 & 60 & 13 & \\
\hline MMA ( $\mu \mathrm{mol} / \mathrm{L})$ & $0.68(0.52-0.89)$ & $0.45(0.26-0.79)$ & $0.18(0.15-0.22)$ & $<0.001$ \\
\hline $\mathrm{tHcy}(\mu \mathrm{mol} / \mathrm{L})$ & $26.0(21.2-31.8)$ & $15.9(10.4-24.2)$ & $10.8(9.3-12.6)$ & $<0.001$ \\
\hline $\begin{array}{l}\mathrm{MMA}>0.75 \mu \mathrm{mol} / \mathrm{L} \text { and tHcy }>15 \\
\mu \mathrm{mol} / \mathrm{L}(\%)\end{array}$ & 32 & 40 & 3 & \\
\hline
\end{tabular}

Abbreviations: HoloTCII, holotranscobalamin II; MMA, methylmalonic acid; tHcy, total homocysteine

${ }^{1}$ Differences in the means between the categories of serum vitamin B12 were tested for by using ANOVA 


\section{DISCUSSION}

The results from this study show that mean serum concentrations of vitamin B12 reflected the level of intake of meat and animal products; omnivores had the highest concentrations of serum vitamin B12 and vegans the lowest. In addition, over half of the men consuming a vegan diet and $7 \%$ of men consuming a vegetarian diet were categorized - according to their serum vitamin B12 concentrations - as being vitamin B12 deficient. Very few men were categorized as being folate deficient (only two omnivores).

The finding that vegans have lower serum concentrations of vitamin B12 and a greater prevalence of biochemical vitamin B12 deficiency than both omnivores and vegetarians is in agreement with results from several other studies [3, 4, 19]. However, the results from some but not all studies have shown that vegetarians have lower vitamin B12 concentrations compared to omnivores and a greater proportion of vegetarians had serum vitamin B12 concentrations indicative of deficient or depleted levels [3-10]. The results from this study do not support the idea that vegetarians, because they consume some animal products, are not at risk of developing a vitamin B12 deficiency [20].

These results showed no evidence that serum vitamin B12 concentrations decreased with increasing duration on a vegetarian or vegan diet, which is in agreement with results from one other small study [21], but not with another, in which an inverse correlation between serum vitamin B12 concentrations and time on a vegan diet $(r=-0.18, P=0.047)$ was reported[11]. Evidence from an intervention study suggests that when the intake of vitamin B12 is restricted, serum concentrations of vitamin B12 may decline much more rapidly when than previously believed. In 13 lacto-ovo vegetarians, mean serum vitamin B12 concentrations were reduced from $345 \mathrm{pmol} / \mathrm{l}$ to $226 \mathrm{pmol} / \mathrm{l}$ within two months of consuming a vegan diet [22]. Only two men in this study had recently started consuming a vegan diet ( $<1$ year) and therefore, the effect on vitamin B12 concentrations of a short term dietary change could not be assessed. It is possible that once vitamin B12 stores decline to a certain level, serum concentrations are maintained despite a very low intake over a prolonged period via a number of mechanisms that might include an increased absorption of vitamin B12 in the gut, reduced vitamin B12 excretion, and an increased capacity to absorb recycled vitamin B12 from the bile via the enterohepatic circulation [20].

Another group at risk of becoming vitamin B12 deficient is the elderly because of a greater prevalence of gastric atrophy and hypochlorhydria, both of which lead to an insufficient absorption of food bound vitamin B12 [13]. Indeed, others have reported a decrease in average serum vitamin B12 concentrations with advancing age in elderly populations ( $>65$ years) [23-27]. There was no evidence of an age-related decline in serum concentrations of vitamin B12 among any of the diet groups in this study, which is similar to that reported in the United Kingdom National Diet and Nutrition Survey [17] ; this may be because these studies did not include many elderly participants.

The finding that over $95 \%$ of vegans and $31 \%$ of vegetarians who were not using supplements failed to meet the RNI for daily vitamin B12 intake $(1.5 \mu \mathrm{g} /$ day from foods) was similar to that reported by others $[12,28]$. Furthermore, even though vegetarian and vegan men who reported taking a vitamin B12 supplement had a higher intake of vitamin 
B12, serum concentrations of vitamin B12 were not affected. It is possible that supplement use was not accurately reported, some of the vitamin B12 supplements taken contained a type of inactive plant based vitamin B12 [29] or that a proportion of men taking a vitamin B12 supplement had been recently diagnosed with a vitamin B12 deficiency. There appears to be a degree of awareness among these participants of the need to supplement vegan and vegetarian diets, inasmuch as $20 \%$ of vegans and vegetarians reported taking supplements regularly. However, because there was little difference in serum vitamin B12 between supplement and non-supplement users, it may be necessary to improve the understanding of the need to regularly consume supplements containing adequate amounts of the active form of vitamin B12.

Subclinical abnormalities are thought to emerge when serum concentrations of vitamin B12 fall below $111 \mathrm{pmol} / \mathrm{l}$ [20]. Over half of the vegans had serum vitamin B12 concentrations below $118 \mathrm{nmol} / \mathrm{L}$ and thus, these men may have a higher risk of developing clinical symptoms related to vitamin B12 deficiency. Evidence of severe neurological damage as result of chronic vitamin B12 deficiency in vegans and vegetarians is rare; however, several case reports have shown neurological impairment that includes irreversible degeneration of the spinal cord, as well as difficulty in walking and handling utensils [30, 31]. There are very few studies that have looked for milder neurological symptoms among vegetarians and vegans who are vitamin B12 deficient.

In this study, serum concentration of vitamin B12 was used as a biomarker of vitamin B12 status, although recent evidence suggests that serum holoTC may be a more sensitive and specific biomarker of vitamin B12 status [32]. HoloTC is the fraction of vitamin B12 bound to transcobalamin that delivers vitamin B12 to cells that synthesize DNA and is thus considered the physiologically active component of vitamin B12[33]. The concentration of holoTC, MMA and tHcy were measured in a subgroup of the study participants; approximately $80 \%$ of men with serum vitamin B12 concentrations below the cut-point of $118 \mathrm{pmol} / \mathrm{I}$ had values for holoTC indicative of vitamin B12 deficiency. On the other hand, only a third of these men had values for both MMA and tHcy above the cut-points considered as biochemically vitamin B12 deficient. Moreover, although the cut-off points used in this study to identify those at risk of vitmanin B12 deficiency have been used elsewhere[17], others have used a higher cutoff point $(250 \mathrm{pmol}, \mathrm{l})$ to define vitamin B12 deficiency [34]. Therefore it is possible therefore that the true proportion of individuals with a vitamin B12 deficiency reported herein has been underestimated.

In conclusion, the results from this study show that vegetarians and vegans have much lower concentrations of serum vitamin B12, but higher concentrations of folate in comparison to omnivores. Mean serum vitamin B12 was not associated with the duration of adherence to a vegetarian or vegan diet, which may indicate that mechanisms that maintain circulating concentrations of vitamin B12 are up-regulated in vegetarians and vegans. Further research into the health effects of vitamin B12 deficiency and depletion in vegans and vegetarians is warranted, and vegetarians and vegans should ensure a regular intake of sufficient vitamin B12 from fortified foods and/or supplements. 


\section{REFERENCES}

1. Key, T.J., et al., Mortality in vegetarians and nonvegetarians: detailed findings from a collaborative analysis of 5 prospective studies. Am J Clin Nutr, 1999. 70(3 Suppl): p. 516S-524S.

2. Key, T.J., et al., Mortality in British vegetarians: results from the European Prospective Investigation into Cancer and Nutrition (EPIC-Oxford). Am J Clin Nutr, 2009. 89(5): p. 1613S-1619S.

3. Majchrzak, D., et al., B-vitamin status and concentrations of homocysteine in Austrian omnivores, vegetarians and vegans. Ann Nutr Metab, 2006. 50(6): p. 485-91.

4. Krajcovicova-Kudlackova, M., et al., Homocysteine levels in vegetarians versus omnivores. Ann Nutr Metab, 2000. 44(3): p. 135-8.

5. Herrmann, W., et al., Vitamin B-12 status, particularly holotranscobalamin II and methylmalonic acid concentrations, and hyperhomocysteinemia in vegetarians. Am J Clin Nutr, 2003. 78(1): p. 131-6.

6. Mann, N.J., et al., The effect of diet on plasma homocysteine concentrations in healthy male subjects. Eur J Clin Nutr, 1999. 53(11): p. 895-9.

7. Hung, C.J., et al., Plasma homocysteine levels in Taiwanese vegetarians are higher than those of omnivores. J Nutr, 2002. 132(2): p. 152-8.

8. Hokin, B.D. and T. Butler, Cyanocobalamin (vitamin B-12) status in Seventh-day Adventist ministers in Australia. Am J Clin Nutr, 1999. 70(3 Suppl): p. 576S-578S.

9. Herrmann, W., et al., Total homocysteine, vitamin $\mathrm{B}(12)$, and total antioxidant status in vegetarians. Clin Chem, 2001. 47(6): p. 1094-101.

10. Haddad, E.H., et al., Dietary intake and biochemical, hematologic, and immune status of vegans compared with nonvegetarians. Am J Clin Nutr, 1999. 70(3 Suppl): p. 586S-593S.

11. Waldmann, A., et al., Homocysteine and cobalamin status in German vegans. Public Health Nutr, 2004. 7(3): p. 467-72.

12. Koebnick, C., et al., Long-term ovo-lacto vegetarian diet impairs vitamin B-12 status in pregnant women. J Nutr, 2004. 134(12): p. 3319-26.

13. Baik, H.W. and R.M. Russell, Vitamin B12 deficiency in the elderly. Annu Rev Nutr, 1999. 19: p. 357-77.

14. Davey, G.K., et al., EPIC-Oxford: lifestyle characteristics and nutrient intakes in a cohort of 33883 meat-eaters and 31546 non-meat-eaters in the UK. Public Health Nutr, 2003. 6(3): p. 259-69.

15. Bingham, S.A., et al., Comparison of dietary assessment methods in nutritional epidemiology: weighed records v. $24 \mathrm{~h}$ recalls, food-frequency questionnaires and estimated-diet records. Br J Nutr, 1994. 72(4): p. 619-43.

16. Bingham, S.A., et al., Validation of weighed records and other methods of dietary assessment using the $24 \mathrm{~h}$ urine nitrogen technique and other biological markers. Br J Nutr, 1995. 73(4): p. 531-50.

17. Ruston, D., et al., National Diet and Nutrition Survey: adults aged 19 to 64 years. Volume 4: Nutritional status (anthropometry and blood analytes), blood pressure and physical activity. 2004: TSO:London.

18. Lloyd-Wright, Z., et al., Holotranscobalamin as an indicator of dietary vitamin B12 deficiency. Clin Chem, 2003. 49(12): p. 2076-8.

19. Obeid, R., et al., The impact of vegetarianism on some haematological parameters. Eur J Haematol, 2002. 69(5-6): p. 275-9.

20. Herbert, V., Staging vitamin B-12 (cobalamin) status in vegetarians. Am J Clin Nutr, 1994. 59(5 Suppl): p. 1213S-1222S.

21. Rauma, A.L., et al., Vitamin B-12 status of long-term adherents of a strict uncooked vegan diet ("living food diet") is compromised. J Nutr, 1995. 125(10): p. 2511-5.

22. Crane, M.G., et al., Vitamin B12 studies in total vegetarians (vegans). Journal of Nutritional and Environmental Medicine 1994(4): p. 419.

23. Wahlin, A., et al., Reference values for serum levels of vitamin B12 and folic acid in a population-based sample of adults between 35 and 80 years of age. Public Health Nutr, 2002. 5(3): p. 505-11.

24. Nilsson-Ehle, H., et al., Serum cobalamins in the elderly: a longitudinal study of a representative population sample from age 70 to 81. Eur J Haematol, 1991. 47(1): p. 10-6.

25. Loikas, S., et al., Vitamin B12 deficiency in the aged: a population-based study. Age Ageing, 2007. 36(2): p. 177-83.

26. Green, T.J., et al., Serum vitamin B12 concentrations and atrophic gastritis in older New Zealanders. Eur J Clin Nutr, 2005. 59(2): p. 205-10. 
27. den Elzen, W.P., et al., Vitamin B12 and folate and the risk of anemia in old age: the Leiden 85-Plus Study. Arch Intern Med, 2008. 168(20): p. 2238-44.

28. Draper, A., et al., The energy and nutrient intakes of different types of vegetarian: a case for supplements? Br J Nutr, 1993. 69(1): p. 3-19.

29. Watanabe, F., Vitamin B12 sources and bioavailability. Exp Biol Med (Maywood), 2007. 232(10): p. 1266-74.

30. Takahashi, H., et al., Subacute combined degeneration of the spinal cord in vegetarians: vegetarian's myelopathy. Intern Med, 2006. 45(10): p. 705-6.

31. Brocadello, F., et al., Irreversible subacute sclerotic combined degeneration of the spinal cord in a vegan subject. Nutrition, 2007. 23(7-8): p. 622-4.

32. Hvas, A.M. and E. Nexo, Holotranscobalamin-a first choice assay for diagnosing early vitamin B deficiency? J Intern Med, 2005. 257(3): p. 289-98.

33. Gibson, R.S., Principles of nutritional assessment. $2^{\text {nd }}$ ed. 2005, New York: Oxford University Press.

34. Lindenbaum, J., et al., Prevalence of cobalamin deficiency in the Framingham elderly population. Am J Clin Nutr, 1994. 60(1): p. 2-11. 


\section{CHAPTER 4}

The Netherlands Cohort Study Meat Investigation Cohort; a population-based cohort over-represented with vegetarians, pescetarians and low meat consumers

Anne MJ Gilsing Matty P Weijenberg R Alexandra Goldbohm Pieter C Dagnelie Piet A van den Brandt and Leo J Schouten

Nutrition Journal 2013;29(12):156 


\section{ABSTRACT}

Background: Vegetarian diets have been associated with lower risk of chronic disease, but little is known about the health effects of low meat diets and the reliability of self-reported vegetarian status. We aimed to establish an analytical cohort over-represented with vegetarians, pescetarians and 1 day/week meat consumers, and to describe their lifestyle and dietary characteristics. In addition, we were able to compare self-reported vegetarians with vegetarians whose status has been confirmed by their response on the extensive food frequency questionnaire (FFQ).

Methods: Embedded within the Netherlands Cohort Study ( $n=120852$; including 1150 selfreported vegetarians), the NLCS-Meat Investigation Cohort (NLCS-MIC) was defined by combining all FFQ-confirmed-vegetarians $(n=702)$, pescetarians $(n=394)$, and 1 day/week meat consumers $(n=1396)$ from the total cohort with a random sample of 2-5 days/weekand 6-7 days/week meat consumers ( $\mathrm{n}=2965$ and 5648, respectively).

Results: Vegetarians, pescetarians, and 1 day/week meat consumers had more favorable dietary intakes (e.g. higher fiber/vegetables) and lifestyle characteristics (e.g. lower smoking rates) compared to regular meat consumers in both sexes. Vegetarians adhered to their diet longer than pescetarians and 1 day/week meat consumers. $75 \%$ of vegetarians with a prevalent cancer at baseline had changed to this diet after diagnosis. $50 \%$ of selfreported vegetarians reported meat or fish consumption on the FFQ. Although the misclassification that occurred in terms of diet and lifestyle when merely relying on selfreporting was relatively small, the impact on associations with disease risk remains to be studied.

Conclusion: We established an analytical cohort over-represented with persons at the lower end of the meat consumption spectrum which should facilitate prospective studies of major cancers and causes of death using $\geq 20.3$ years of follow-up. 


\section{INTRODUCTION}

The popularity of (semi-)vegetarianism is rapidly increasing in the western world as a result of nutritional, ethical, and more recently, environmental concerns [1]. At the same time, the world-wide per capita meat consumption is also expected to increase considerably over the next decades [2], especially in developing countries [3, 4].

Although this transition has been accompanied by an increasing scientific interest in the health effects of vegetarian diets, only a small number of large prospective cohort studies specifically set out to study these by including a large proportion of vegetarians [5-10]. Thus far, these studies provided convincing evidence that vegetarians have a lower risk of coronary heart disease [11], a possibly lower risk of diabetes [12], metabolic syndrome [13], and some types of cancer[14], and a greater life expectancy [11] compared to meat eaters. These observed health benefits may largely be explained by a more favorable distribution of chronic disease risk factors such as overweight [15, 16], blood lipids [17], and blood pressure [18] among the vegetarians.

Despite these scientific advancements, no universally accepted definition for the term vegetarian exists and there is substantial inconsistency in how people self-identify being vegetarian $[19,20]$. This ambiguity may hinder the comparability of previous studies. Only a few studies identified vegetarians based on extensive dietary data collection techniques [5, 6] whereas others reported to have classified vegetarians by means of several broader questions relating to the overall consumption of animal products [7-9], or to have used selfreported vegetarianism [10] as inclusion criterion. Even though it has been shown that selfreported vegetarians are generally health conscious, several studies suggest that this group still includes a considerable number of occasional meat consumers $[19,20]$ which may have biased epidemiological study results. Therefore, the usefulness and reliability of selfreported vegetarian status in observational studies needs to be further evaluated. Moreover, further work is needed in studies with a large number of vegetarians and low meat eaters to address whether complete abstinence of meat might have an additional beneficial health effect over low meat consumption, or vice versa.

Therefore, we have defined an analytical cohort specifically designed to study the health effects of vegetarian and low meat diets focusing on cancer incidence and mortality, embedded within a large ongoing prospective cohort that used an extensive FFQ to assess dietary intake. In the current paper we present the description of the baseline characteristics of this analytical cohort that is overrepresented with low and no meat consumers. We performed a cross-sectional comparison of lifestyle factors, dietary intake, and prevalent cancer status across the various meat consumption categories. In addition, we performed a cross comparison of self-reported vegetarians and vegetarians whose status has been confirmed by their response on the FFQ.

\section{METHODS}

The prospective Netherlands Cohort Study on diet and cancer (NLCS) was initiated in 1986 with the purpose of investigating the association between diet and cancer. The details of the study have been described elsewhere [21]. The study population originated largely 
from 204 municipal population registries throughout the country. In addition, vegetarians were overrepresented by intentionally contacting them through health food shops and magazines. This method of recruitment resulted in the inclusion of 685 vegetarians and low meat consumers. A total of 58279 men and 62573 women between the ages of 55 and 69 years completed a mailed, self-administered questionnaire on dietary habits and other risk factors for cancer, at baseline. The NLCS has been approved by the institutional review boards of the TNO Quality of Life Research Institute (Zeist, the Netherlands) and Maastricht University (Maastricht, the Netherlands). Because the NLCS traditionally uses a case-cohort approach for reasons of efficiency in questionnaire processing, the baseline questionnaires were only entered for all failures (i.e. incident cancer cases) and a random subcohort of 5000 individuals that was chosen immediately after baseline. The first page of the questionnaire was entered and processed for all 120852 participants.

\section{NLCS-MIC}

We defined an analytical cohort, embedded within the NLCS, that is specifically designed to study the health effects of vegetarian and low meat diets; the NLCS 'Meat Investigation Cohort' (NLCS-MIC). The NLCS-MIC was created by expanding the random subcohort of the NLCS from 5000 to 10000 individuals (to increase power) and combining these with all the (self-reported) vegetarians and individuals who consumed meat 1 day/week from the NLCS. The latter groups were identified based on two items on the first page of the questionnaire relating to specific dietary regimens: "how many days on average per week do you eat meat?", and "Do you have any special eating habits?" The remainder of the questionnaire, including the 150 item semi-quantitative food frequency questionnaire (FFQ), was also entered and processed for this group of vegetarians and low meat consumers. The detailed dietary data was used to further categorize NLCS-MIC into five meat consumption categories (non-meat consumers based on FFQ (divided into vegetarians and pescetarians), 1 day/week-, 2-5 days/week-, and 6-7 days/week meat consumers), as described below.

\section{Meat consumption questions}

The NLCS-FFQ that was used to categorize NLCS-MIC, contained 14 items on the consumption of meat with the hot meal (mainly fresh meat, including chicken), 5 items on the consumption of meat products used as sandwich fillings, and 3 items on fish consumption. A validation study conducted in a subgroup of the cohort two years after the baseline measurement indicated that the Spearman correlation coefficients for meat, meat products and fish, as assessed by the questionnaire, and those estimated from the 9-day dietary record were $0.46,0.54$ and 0.53 respectively. The number of vegetarians and individuals consuming meat 1 day/week was too low in this validation sample to assess the above correlations in these selected groups [22]. In addition, the questionnaire also assessed the time since the start of any special eating habits and weekly frequency of meat consumption (for 0-1 day/week meat consumers), in years prior to baseline (1986).

\section{Classification of NLCS-MIC}

NLCS-MIC was classified based on the FFQ as depicted in Supplemental Figure 1.

\section{Classification of non-vegetarians}

Individuals who reported to eat $\geq 1$ type of meat with the hot meal (14 items on FFQ) were categorized based on their self-reported weekly meat consumption frequency as indicated 
in the question ("how many days on average per week do you eat meat?") into 1 day/week, 2-5 days/week, and 6-7 days/week meat consumers.

Classification of "FFQ confirmed vegetarians and pescetarians"

We defined 'FFQ confirmed vegetarians' (later referred to as 'confirmed-vegetarians') as individuals who reported to consume a diet void of meat and fish on the extensive FFQ (including vegans, lactoovo-, lacto-, and ovo-vegetarians). In order to be classified as nonmeat consumers individuals had to fulfill the following three criteria simultaneously: 1 ) not eating any meat items for the hot meal (14 items FFQ), 2) abstaining from meat as sandwich fillings (5 items FFQ), and 3) consuming meat for 0 days/week as indicated in the question ("how many days on average per week do you eat meat?"). In addition, individuals adhering to criteria 1 and 2 with a missing on criterion 3 who indicated to adhere to a nonmeat dietary regimen (i.e. vegetarian/vegan), were also considered to be non-meat consumers. All non-meat consumers were subsequently categorized to be either vegetarian or pescetarian (fish eater) based on the 3 items relating to fish consumption.

\section{Classification of self-reported vegetarians}

Because we wanted to examine the reliability of self-reported vegetarianism as compared to a vegetarian status confirmed by the FFQ, we additionally identified all self-reported vegetarians based on the question: "Do you have any special eating habits?" Individuals who reported to adhere to a vegetarian, vegan, or Seventh Day Adventist diet were classified as self-reported vegetarians.

Due to the case-cohort design of the original NLCS cohort, NLCS-MIC consists of a random sample of 10000 individuals (with random meat consumption), and all the vegetarians and low meat consumers from the total cohort. As a result, all self-reported vegetarians who reported eating meat $>1$ day/week and who were not part of the randomly selected subcohort $(n=119)$, were only included in analyses when comparing self-reported vegetarians to the complementary group of non-(self-reported) vegetarians, and not for all other contrasts

\section{Incomplete and inconsistent dietary data}

Within the NLCS, participants with incomplete and inconsistent dietary data are excluded from analyses [22]. These exclusion criteria were based on the number of blank and marked items, but not designed for use in non-meat eating populations. As vegetarians left meat-related items blank, they automatically meet these exclusion criteria more easily than those who consumed meat in their diet. Therefore, new cut-off values for non-meat consumers, using the same underlying principles as for the total NLCS cohort, were established. For all non-meat consumers, the cut-off criteria for incomplete questionnaires were set at $>90$ blank- and $<22$ consumed items, whereas meat eaters were scored according to the original NLCS values ( $>60$ items and $<35$, respectively). Out of the 135 nonmeat eaters with incomplete questionnaires, 39 were no longer considered to be incomplete after implementing these new scores. The proportion of incomplete questionnaires was $6 \%$ among the random sample of 10,000 and $7 \%$ among the non-meat eaters. Finally, NLCS-MIC consists of 11,867 cohort members, including 1,227 non meat consumers (785 vegetarians and 442 pescetarians) and 1,499 participants who consume meat for only 1 day/week. 


\section{Statistical Analyses}

Dietary and lifestyle characteristics were described for the five meat consumption categories (confirmed-vegetarians and -pescetarians, and individuals consuming meat1 day/week, 2-5 days/week, and 6-7 days/week). We also examined differences in dietary and lifestyle characteristics between the classifications of vegetarian status, i.e. between self-reported and FFQ based vegetarians.

Because of the relatively high number of cancer survivors among the vegetarians, pescetarians and individuals consuming meat 1day/week, all prevalent cancer cases at baseline were excluded from the main analyses and described as a separate group. All analyses were conducted for men and women separately. All nutrient intake variables were adjusted for energy intake by the residual method [23]. Differences between the five meat consumption categories were assessed using chi-square tests for categorical variables, and one-way analyses of variance (ANOVA) for continuous variables.

Due to a skewed distribution of some dietary intake variables we transformed these variables prior to performing analyses of variance, using the log normal transformation. Non-parametric tests were also applied to not normally distributed data (untransformed) (Kruskal-Wallis test), and because these results were comparable to those from ANOVA, the latter was applied to all items for consistency.

All analyses were performed with STATA Statistical Software (Intercooled version 11; StataCorp LP, College Station, TX). All tests were 2-tailed, and differences were regarded as statistically significant at $P<0.05$.

\section{RESULTS}

Table 1: Self-reported vegetarian status at baseline of members of the NLCS-Meat Investigation Cohort (NLCS-MIC) by meat consumption status ${ }^{\mathrm{a}}$.

\begin{tabular}{|c|c|c|c|c|c|c|c|c|}
\hline & & \multicolumn{3}{|c|}{$\begin{array}{l}\text { All confirmed vegetarians and low } \\
\text { meat consumers from the total NLCS } \\
\text { cohort }\end{array}$} & \multicolumn{2}{|c|}{$\begin{array}{l}\text { Random sample of meat } \\
\text { consumers from the } \\
\text { total NLCS cohort }{ }^{b} \\
\end{array}$} & \multirow{2}{*}{ Remainder ${ }^{\mathrm{c}}$} & \multirow[b]{2}{*}{ Tota } \\
\hline & & Vegetarians & Pescetarians & 1 day/wk & $\begin{array}{l}2-5 \\
\text { day/wk }\end{array}$ & $\begin{array}{l}\text { 6-7 } \\
\text { day/wk }\end{array}$ & & \\
\hline \multicolumn{9}{|l|}{ Men } \\
\hline & $\begin{array}{l}\text { Self-reported } \\
\text { vegetarians }\end{array}$ & $194(47 \%)^{d}$ & $96(23 \%)$ & $71(17 \%)$ & $5(1 \%)$ & $1(0.3 \%)$ & $49(12 \%)$ & 416 \\
\hline & $\begin{array}{l}\text { Non self-reported } \\
\text { vegetarians }\end{array}$ & $23(0.5 \%)$ & $56(1 \%)$ & $411(9 \%)$ & $1321(28 \%)$ & $2972(62 \%)$ & $\mathrm{N} / \mathrm{A}$ & \\
\hline & Total & 217 & 152 & 482 & 1326 & 2973 & 49 & \\
\hline \multirow[t]{3}{*}{ Women } & $\begin{array}{l}\text { Self-reported } \\
\text { vegetarians }\end{array}$ & $380(52 \%)$ & $151(21 \%)$ & $132(18 \%)$ & $7(1 \%)$ & $4(0.6 \%)$ & $60(8 \%)$ & 734 \\
\hline & $\begin{array}{l}\text { Non self-reported } \\
\text { vegetarians }\end{array}$ & $105(2 \%)$ & $91(2 \%)$ & $782(15 \%)$ & $1632(31 \%)$ & $2672(51 \%)$ & N/A & \\
\hline & Total & 485 & 242 & 914 & 1639 & 2676 & 60 & \\
\hline Total & & 702 & 394 & 1396 & 2965 & 5649 & 109 & \\
\hline
\end{tabular}




\section{The NLCS-MIC}

After excluding prevalent cancer cases at baseline, the NLCS-MIC includes 1150 selfreported vegetarians, of whom $50 \%$ were also classified as vegetarian according to the information on the FFQ ( $n=574 ; 194$ men and 380 women); the remaining $50 \%$ of selfreported vegetarians reported to eat meat and/or fish on the FFQ. Moreover, an additional 23 males and 104 females were classified as vegetarians according to the FFQ but did not define themselves as being vegetarian, resulting in a total of 702 confirmed-vegetarians (Table 1). In addition, the NLCS-MIC also includes 394 pescetarians, 1,396 individuals consuming meat only 1 day/week, 2,965 individuals eating meat 2-5 days/week and 5,649 individuals who consume meat six or seven days/week (Supplemental Figure 1).

\section{Comparison of the five diet groups based on the FFQ}

Data on lifestyle and reproductive characteristics by meat consumption category are presented in Table 2. No difference in age was observed between the diet groups. Vegetarians and pescetarians were more often female. Mean BMI was lowest amongst pescetarians and vegetarians and increased with increasing meat intake. In both men and women, those who reported not to consume meat had, on average, a higher level of education, and were least likely to be married or current smokers. Age at menarche was lowest in the vegetarian women. On average, vegetarians had the lowest number of children (data only available for women). Among men, those who reported not to consume meat had more frequent bowel movements compared to meat eaters $(P<0.001)$, while fish eaters had the lowest rates of constipation $(P<0.001)$ (data only available for men) (Table 3). Among women, vegetarians and pescetarians more often considered their own health status to be excellent (20\%) compared to all the meat eating groups ( 14\%).

Detailed data on intake (in g/day) of major food groups, selected foods and macronutrients in the five meat consumption groups are shown in Table 4; intake of all items was significantly different across the groups $(P<0.001)$ in both men and women. In short, total energy intake was lowest in those who reported to consume meat 1 day/week, followed by vegetarians, pescetarians, and highest in individuals consuming meat more than 2 days/week. The vegetarians and pescetarians consumed the largest amounts of fruits, vegetables, pulses, grains, nuts and seeds, soy products, dairy and cheese; consumption of these products was lowest among 2-5 and 6-7 days/week meat eaters, while individuals who reported to consume meat 1 day/week had intakes at in-between levels. Mean fish intake decreased from the low to high meat intake groups and was highest amongst the pescetarians. Individuals in the highest meat eating group (6-7 days/week) consumed alcohol at levels almost three times as high as the vegetarians.

Data on daily intake of vitamins and minerals in the five meat consumption groups are shown in Table 5. Intake of all these micronutrients was significantly different across the diet groups $(P<0.001)$. Intake of vitamins A, B2, C, E, carotene, calcium, folate, phosphorus, and dietary fiber was highest among the vegetarians and decreased with increasing intake of meat. The opposite pattern was observed for intake of vitamin B6 and cholesterol in the diet which was highest among those consuming meat 6-7 days/week. The amount of salt added to the meal during cooking increased considerably with increasing meat intake; individuals consuming meat 6-7 days/week added nearly three times the amount of salt compared to vegetarians. 


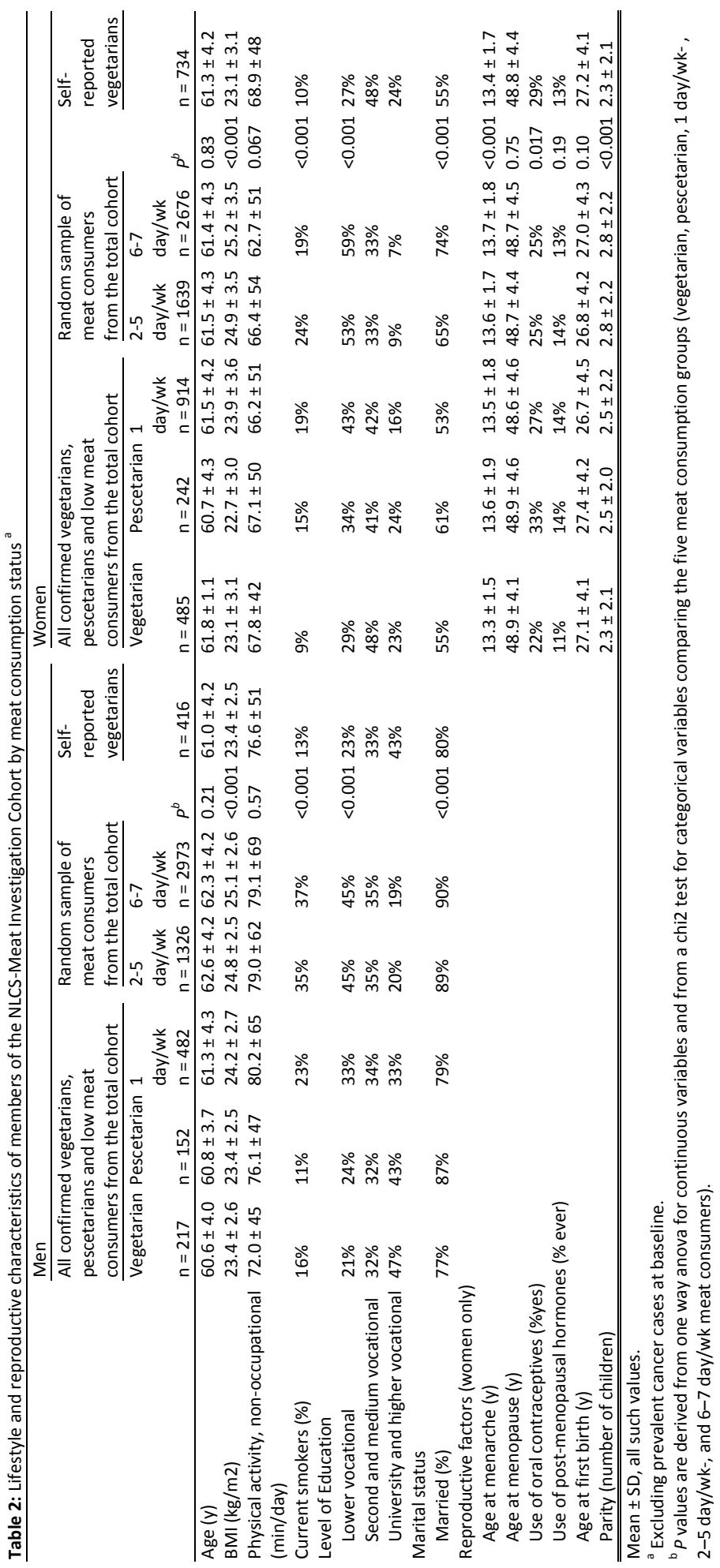




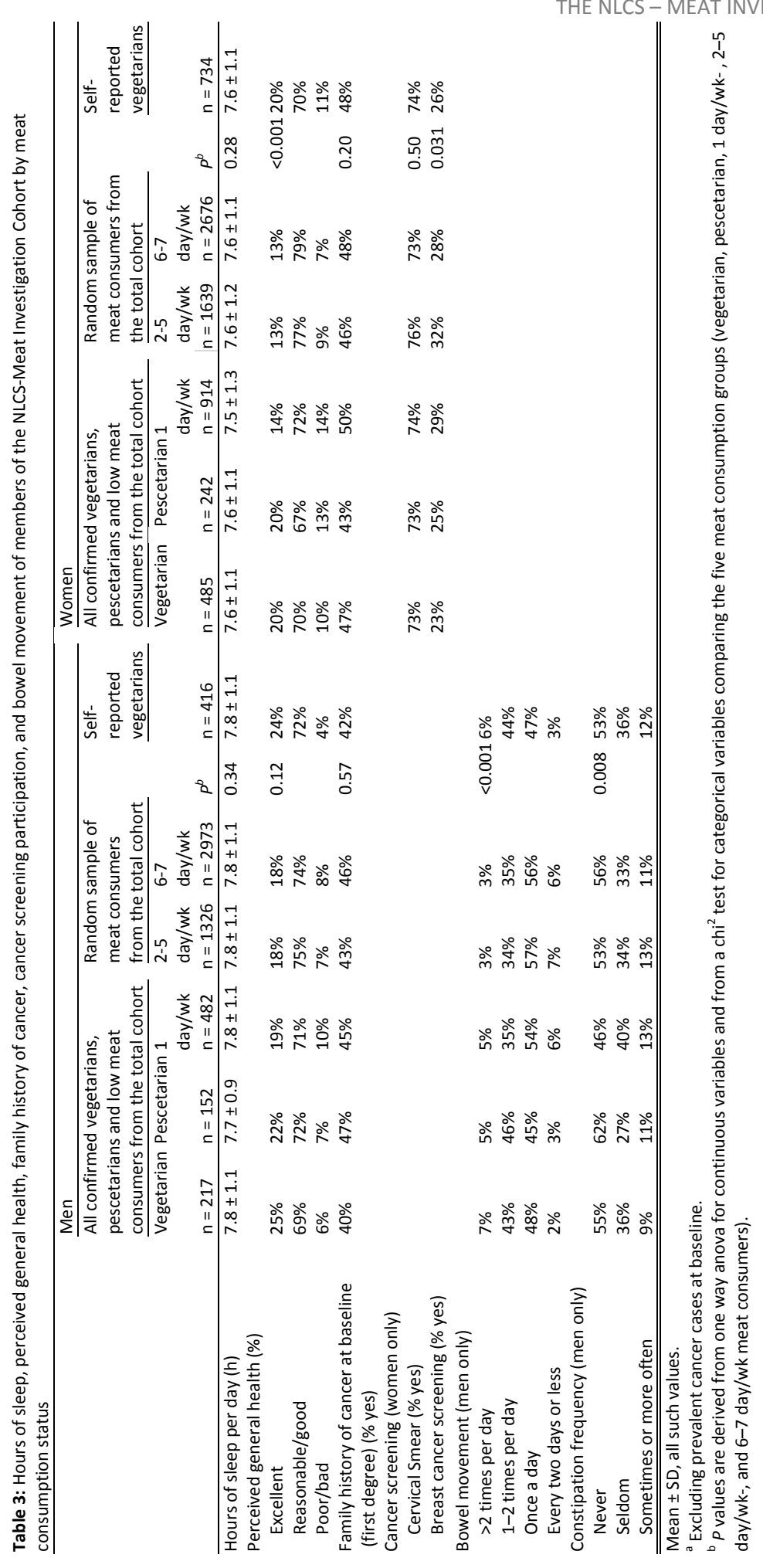




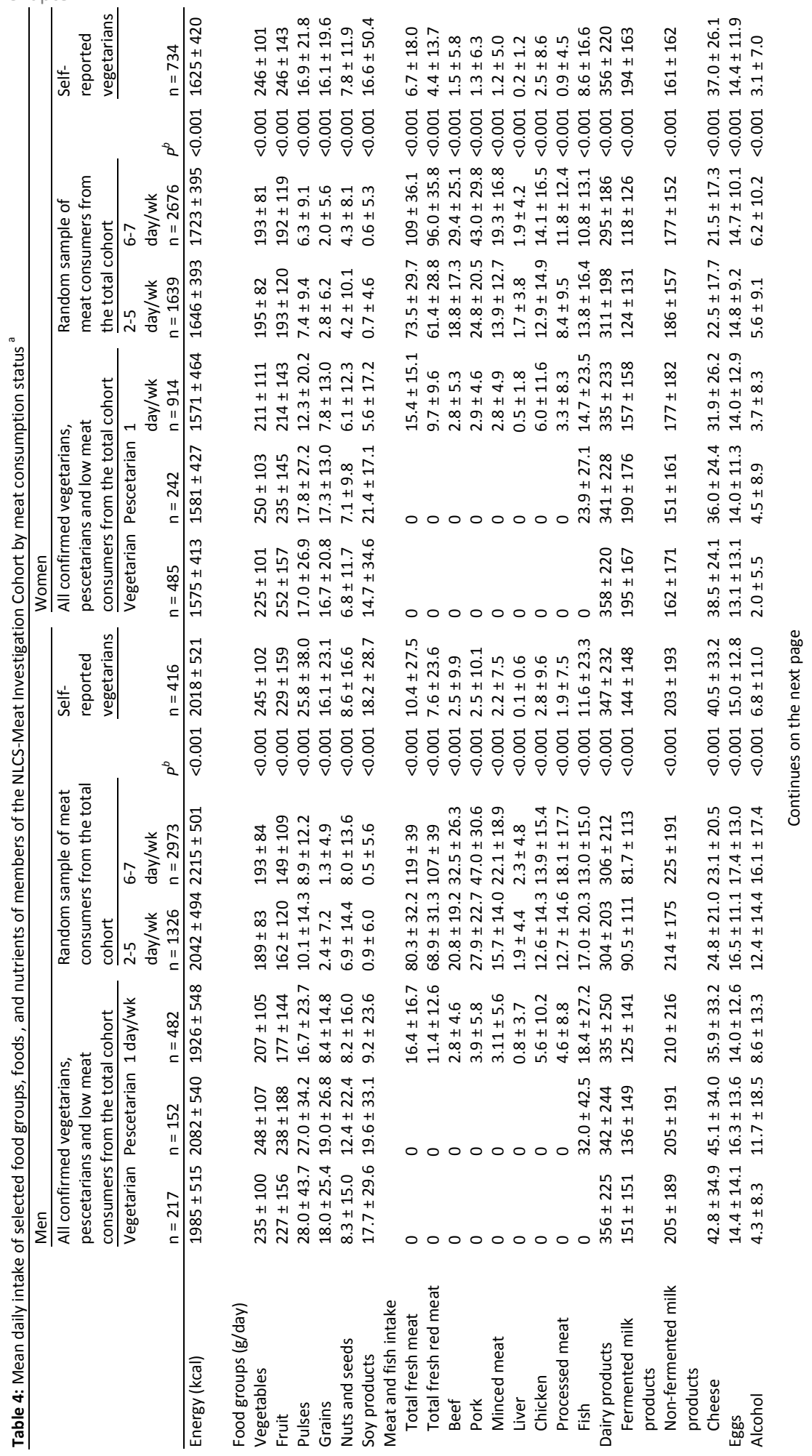




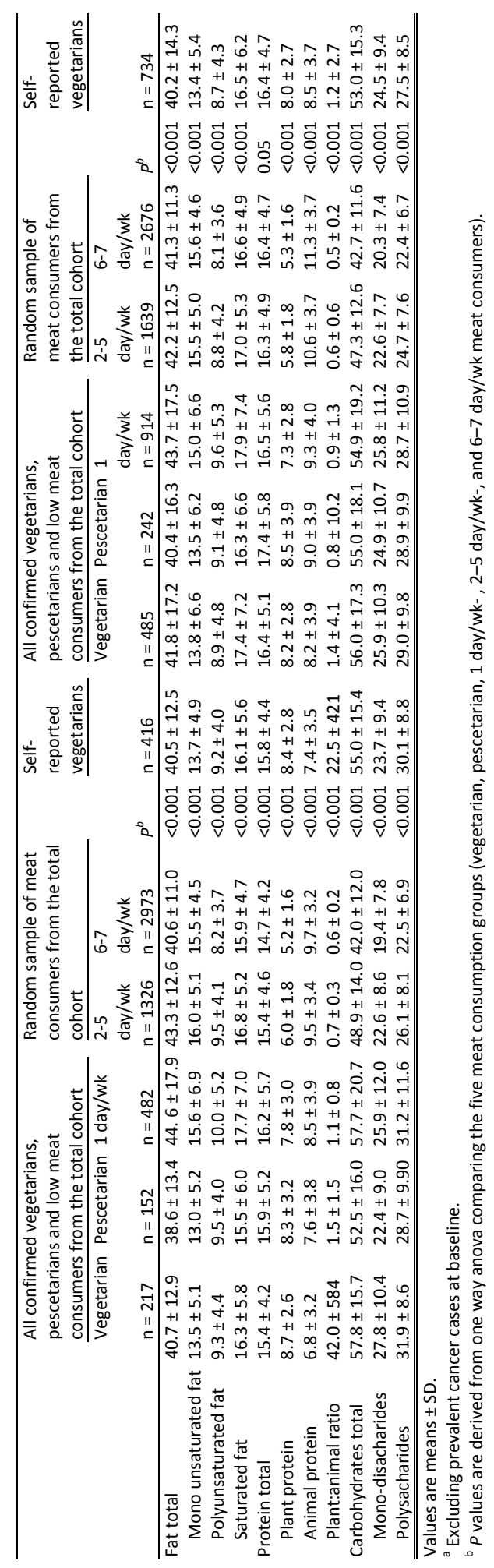




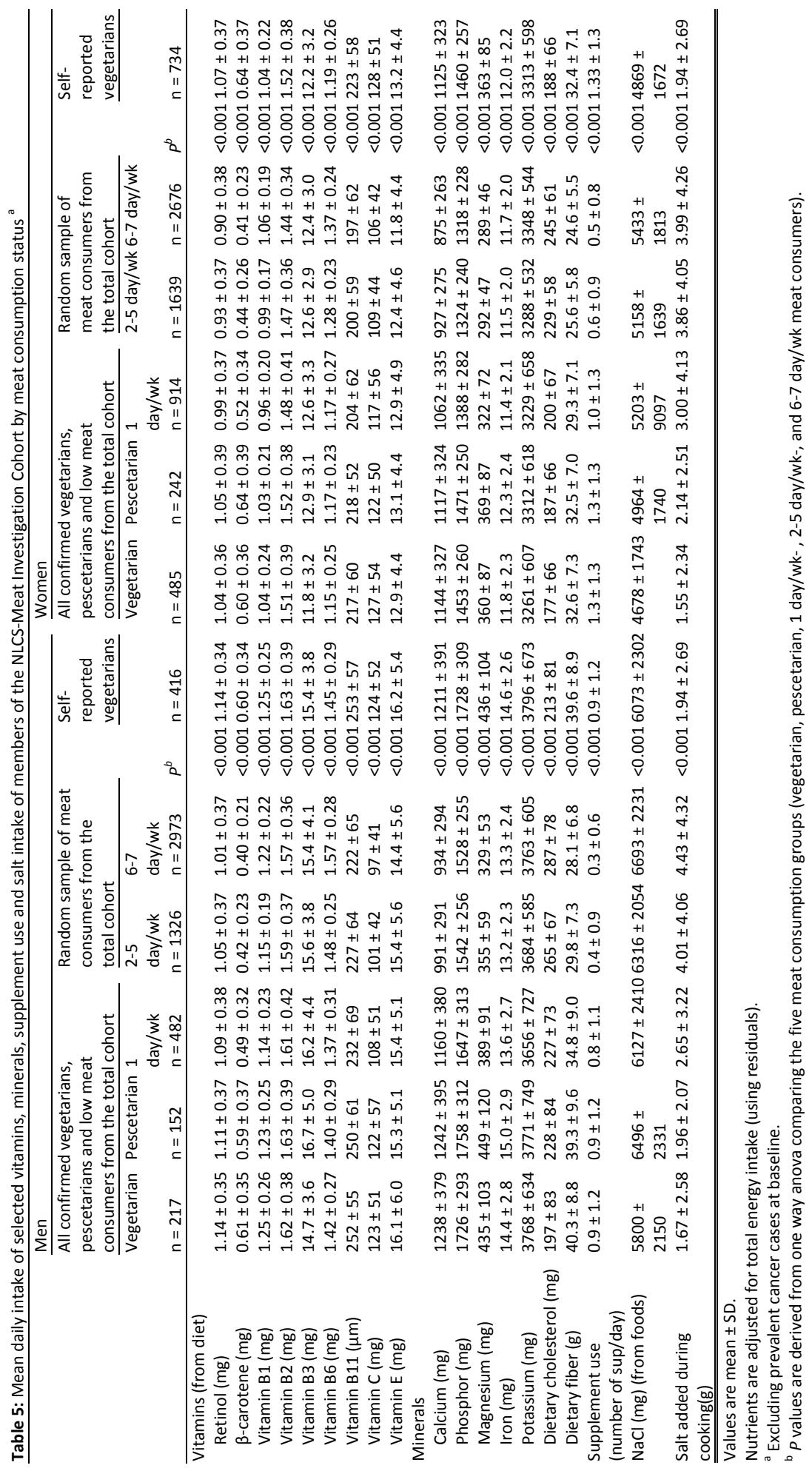




\section{Time on diet}

The time of adherence to diet was significantly different between vegetarians, pescetarians and those consuming meat 1 day/week $(P<0.001)$ (Table 6). Both vegetarian men and women were more often on a dietary regimen for a longer period of time $(>15 y)$ than pescetarians and low meat consumers.

Table 6: Time on diet by meat consumption status ${ }^{\mathrm{a}}$

\begin{tabular}{|c|c|c|c|c|c|c|c|c|c|c|}
\hline & \multicolumn{5}{|l|}{ Men } & \multicolumn{5}{|l|}{ Women } \\
\hline & \multicolumn{4}{|c|}{$\begin{array}{l}\text { All confirmed vegetarians, } \\
\text { pescetarians and low meat } \\
\text { consumers from the total cohort }\end{array}$} & \multirow[t]{2}{*}{$\begin{array}{l}\text { Self- } \\
\text { reported } \\
\text { vegetarians } \\
\end{array}$} & \multicolumn{4}{|c|}{$\begin{array}{l}\text { All confirmed vegetarians, } \\
\text { pescetarians and low meat } \\
\text { consumers from the total cohort }\end{array}$} & $\begin{array}{l}\text { Self- } \\
\text { reported } \\
\text { vegetarians } \\
\end{array}$ \\
\hline & \multirow{2}{*}{$\begin{array}{l}\text { Vegetarian } \\
\mathrm{n}=217\end{array}$} & \multicolumn{3}{|c|}{$\begin{array}{r}\text { Pescetarian } 1 \\
\text { day/wk }\end{array}$} & & \multicolumn{4}{|c|}{$\begin{array}{r}\text { Vegetarian Pescetarian } 1 \\
\text { day/wk }\end{array}$} & \multirow[b]{2}{*}{$n=734$} \\
\hline & & $\mathrm{n}=152$ & $\mathrm{n}=48$ & $P^{\mathrm{b}}$ & $n=416$ & $\mathrm{n}=485$ & $n=242$ & $\mathrm{n}=914$ & $P^{\mathrm{b}}$ & \\
\hline \multicolumn{11}{|c|}{$\begin{array}{l}\text { Time of adherence to } \\
\text { special eating habit (\%) }\end{array}$} \\
\hline$\leq 5 y$ & $9 \%^{c}$ & $28 \%$ & $27 \%$ & $<0.001$ & $18 \%$ & $16 \%$ & $33 \%$ & $27 \%$ & $<0.0$ & $21 \%$ \\
\hline $6-10 y$ & $23 \%$ & $27 \%$ & $22 \%$ & & $23 \%$ & $25 \%$ & $27 \%$ & $26 \%$ & & $25 \%$ \\
\hline $11-15 y$ & $18 \%$ & $13 \%$ & $14 \%$ & & $16 \%$ & $16 \%$ & $16 \%$ & $14 \%$ & & $16 \%$ \\
\hline$\geq 16 y$ & $44 \%$ & $25 \%$ & $23 \%$ & & $34 \%$ & $37 \%$ & $17 \%$ & $19 \%$ & & $30 \%$ \\
\hline No data & $7 \%$ & $7 \%$ & $14 \%$ & & $9 \%$ & $6 \%$ & $7 \%$ & $14 \%$ & & $9 \%$ \\
\hline
\end{tabular}

\section{Prevalent cancer cases (results not shown)}

The proportion of prevalent cancer cases was highest among the vegetarian and pescetarians (both $11 \%$ ) and significantly decreased with increasing meat intake (1 day/week (7\%), 2-5 days/week (5\%), and 6-7 days/week $(4 \%)(P<0.001)$ (Supplemental Figure 1). Vegetarians who had been diagnosed with cancer before baseline (prevalent cancer cases) were more often female and showed a more favorable distribution of lifestyle factors compared to vegetarians without a cancer diagnosis; they were more physically active and less likely to be current smokers. As could be expected, prevalent cancer cases scored their general health status considerably lower, and spent more hours per day sleeping. Vegetarians with a prevalent cancer were more extreme in their dietary intakes compared to those without a cancer diagnosis; they ate substantially more fruits, vegetables, pulses, grains, nuts and seeds, dairy cheese, eggs, proteins and carbohydrates, but less fat and alcohol. Moreover, only $65 \%$ of vegetarians with a prevalent cancer reported using a nutritional supplement compared to $58 \%$ in the cancer free vegetarians. Three quarters of the vegetarians and 1 day/week meat consumers with a cancer diagnosis at baseline had started their dietary regimen in the same year or the year following their cancer diagnosis. This was also reflected in their shorter time of adherence to their special eating habits compared to cancer free individuals.

\section{Cross-comparison of self-reported vegetarian status and FFQ data}

We observed that $50 \%$ of all self-reported vegetarians reported meat consumption on the FFQ. However, self-reported vegetarians who reported meat of fish consumption on the FFQ did not differ much with respect to lifestyle and dietary characteristics compared to self-reported vegetarians who abstained from all meat and fish products. Moreover, the 
self-reported vegetarians who reported meat consumption on the FFQ ate very little meat at rates comparable to the 1 day/week meat consumers (results not shown). Among women, compared to self-reported vegetarians who reported meat or fish consumption on the FFQ, confirmed vegetarians were significantly less often current smokers ( $15 \%$ and $6 \%$, respectively $(P<0.001)$, results not shown). Although confirmed self-reported vegetarians had a higher level of education $(P<0.001)$ compared to the self-reported vegetarians who reported meat or fish consumption on the FFQ, this latter group still had a higher education level than any of the meat consumption groups (results not shown). Meat eating selfreported vegetarians had higher intakes of vitamin B3, B6, cholesterol and a lower intake of dietary fiber, and added more salt to their meal during cooking compared to confirmed self-reported vegetarians $(P<0.001)$ (results not shown).

\section{DISCUSSION}

We defined an analytical cohort with a wide range of dietary intake by over-representing our study population with persons at the lower end of the meat consumption spectrum. The dietary intake patterns and lifestyle characteristics of vegetarians, pescetarians and those consuming meat 1 day/week were diverse and distinct from individuals consuming meat on a regular basis. A cross-comparison between self-reported vegetarians and vegetarians whose status was confirmed based on extensive FFQ data, did not show large differences between both groups in terms of diet and lifestyle characteristics.

To date, no universally accepted definition for the term vegetarian exists and the only constant component in a vegetarian diet across all previous empirical studies has been the absence of meat. As previously outlined by Fraser, the problem of defining a dietary regimen on just one food group is that it results in a lack of control on intake of all other food groups that make up a vegetarian diet [24]. Consequently, all individuals who refrain from meat, but have otherwise quite distinct dietary intakes, are grouped together under one label of vegetarianism. We included a large number of individuals consuming meat only once a week with the purpose to address whether low meat consumption might have a beneficial health effect over complete abstinence of meat, or vice versa. Despite an increase in the number of low meat consumers and meat reducers in the Western world $[25,26]$, little to no research has been conducted in this area. However, we showed no large differences in terms of nutrient intake between these groups, though we had no data on vitamin B12 intake [27].

Interestingly, we showed that the perceived general health of vegetarians is considerably better than that of non-vegetarians. This is in line with our finding that vegetarianism is not merely characterized by a diet void of all flesh foods, but rather extends into a complete healthy lifestyle. In our population, we found evidence for a higher level of health consciousness among the vegetarians, pescetarians and to a lesser extent low meat consumers, as indicated by a more favorable distribution of dietary and lifestyle factors. Although the diet groups were statistical significantly different with respect to most of the dietary variables, this may have resulted from our large sample size. Interestingly, the amount of salt added to the food during cooking increased with increasing animal product intake in a dose-response relation. Pescetarians had higher intakes of soy products, nuts and seeds, grains, cheese and eggs than vegetarians, which would suggest that they more 
actively seek to replace the meat in the diet with plant based protein-rich foods to balance their diets.

Appropriately planned vegetarian diets have shown to be consistent with the current dietary guidelines in all stages of the lifecycle [28]. The mean nutrient intakes (not from supplements) in all diet groups were generally well above the recommended daily allowances (RDA) of the European Commission [29], except for iron for which the intakes were below the RDA of $14 \mathrm{mg} /$ day in the meat eating men and all women. The estimated iron intake was highest among pescetarians and vegetarians. However, these groups consume predominantly inorganic iron which has a low bioavailability compared to the heme-bound iron that is found in meat products [30]. More than $55 \%$ of the vegetarians and pescetarians reported to take a nutritional supplement over the last year compared to $26 \%$ of individuals in the highest meat consumption group (6-7 days/week). This suggests that there appears to be a certain degree of awareness among these individuals of the need to supplement their diets to prevent dietary deficiencies.

Although literature suggests that long-term adherence to a vegetarian diet appears more strongly associated with health outcomes than short term adherence [11, 31], very few prospective studies in vegetarian populations have specific data available on time since adopting the diet. On average, vegetarians adhered to their diet considerably longer than pescetarians and individuals consuming meat 1 day/week. As much as $75 \%$ of all confirmed-vegetarians who had been diagnosed with cancer before baseline changed to this dietary regimen after diagnosis. In addition, the proportion of prevalent cancer cases decreased with increasing meat intake. Previous research indicates that cancer survivors are highly motivated to make dietary changes towards a more plant-based diet after diagnosis with the intention to improve their health and well-being [32]. However, although nutrition has shown to affect cancer progression [33], it remains to be elucidated whether adopting a vegetarian lifestyle may influence the course of cancer prognosis or cancer recurrence.

Nearly half of those who called themselves vegetarian reported to consume fish, meat or poultry on the extensive FFQ ( $\mathrm{k}=0.59)$. Similar findings have been reported previously [19, 20] and indicate that the complete avoidance of meat cannot be assumed among selfreported vegetarians. This suggests that self-identification is not a good measure for estimating the prevalence of vegetarianism. However, our findings suggest that the level of misclassification that occurs when merely relying on self-reported vegetarian status was small: the overall group of self-reported vegetarians did not differ considerably from individuals whose vegetarian status has been confirmed based on FFQ data in terms of diet and lifestyle. The FFQ assessed the diet in the past 12 months whereas self-definition was based on vegetarian status at the time of the questionnaire. Nonetheless, only $10 \%$ of the observed disagreement could be explained by this difference in time-frame. It would be interesting to examine the difference in risk ratios between both groups of vegetarians in future etiological studies of chronic diseases. After 20.3y of follow-up, a total of 1559 incident cancer cases (165 among vegetarians and 346 among 1 day/week meat consumers) were identified in NLCS-MIC through record linkage with the Netherlands Cancer Registry. 
An important methodological issue when comparing previous studies on the health effects of vegetarian diets relates to between-study sampling differences. Only a few reports on vegetarian diets, including ours, are from population-based studies while the majority stem from convenience samples that have likely also recruited more health-conscious nonvegetarians $[5-8,10,34]$. The latter technique is particularly appropriate to recruit non vegetarians who only differ from vegetarians with respect to their meat and fish intake and is a suitable design for studies into diet and health in vegetarian populations that are mainly concerned with the adverse effects of meat. However, interest has shifted towards the health effects of the complete vegetarian lifestyle. For this, convenience sampling may be less appropriate since it likely decreases diet and lifestyle differences between vegetarians and non-vegetarians, and could bias results towards the null. Our population has a wide distribution of nutrient intakes and lifestyle characteristics, which should facilitate the identification of associations between vegetarianism, meat consumption and disease risk in future etiologic studies. The ratio of low meat consumers to high meat consumers (meat consumption $\leq 1$ day/week versus 6-7 days/week) was 1:23 in the total NLCS cohort and 1:2.2 in NLCS-MIC.

The NLCS aimed to overrepresent vegetarians by intentionally contacting them through health food shops and magazines. Therefore, vegetarian dietary patterns were taken into consideration when designing the FFQ by including line items on meat substitutes that were commonly used by the vegetarian population at that time. Vegetarians and low meat consumers more often took the opportunity to report and give details of foods and beverages that were frequently eaten but that were not contained in the FFQ. Moreover, vegetarian status was taken into account for nutrient calculation of composite recipes. Previous studies indicate that vegetarians are able to recall their diet with higher reliability [35] but at the same time may be more tempted to report the intake of certain food items that they consider to be healthy as a result of social desirability bias [20]. The FFQ used in the NLCS was not designed for assessing the usefulness of self-reported vegetarianism as a classification tool. Interestingly, some $18 \%$ of individual who were classified as vegetarians based on their responses on the FFQ did not report to have any special eating habits. This phenomenon has previously been reported [20] and may result from lack of knowledge of the concept of vegetarianism by the general public at the time the measurement was conducted (1986).

With NLCS-MIC we successfully established an analytical cohort comprising a considerable number of vegetarians, pescetarians, and 1 day/week meat consumers. The wide distribution of dietary and lifestyle characteristics within the cohort should facilitate the identification of associations between vegetarianism, meat consumption and the risk of major types of cancer and cause-specific mortality using $\geq 20.3$ y of follow-up in future etiologic studies.

\section{REFERENCES}

1. Fox, N. and K. Ward, Health, ethics and environment: a qualitative study of vegetarian motivations. Appetite, 2008. 50(2-3): p. 422-9.

2. FAO, World Agriculture:Towards 2030/2050. Interim Report: Prospects for Food, Nutrition, Agriculture and Major Commodity Groups 2006, (Rome:Global Perspective Studies Unit, Food and Agriculture Organization of the United Nations). 
3. Gill, M., Meat production in developing countries. Proc Nutr Soc, 1999. 58(2): p. 371-6.

4. Steinfeld, H., The livestock revolution-a global veterinary mission. Vet Parasitol, 2004. 125(1-2): p. 19-41.

5. Beeson, W.L., et al., Chronic disease among Seventh-day Adventists, a low-risk group. Rationale, methodology, and description of the population. Cancer, 1989. 64(3): p. 570-81.

6. Butler, T.L., et al., Cohort profile: The Adventist Health Study-2 (AHS-2). Int J Epidemiol, 2008. 37(2): p. 260-5.

7. Frentzel-Beyme, R., J. Claude, and U. Eilber, Mortality among German vegetarians: first results after five years of follow-up. Nutr Cancer, 1988. 11(2): p. 117-26.

8. Appleby, P.N., et al., The Oxford Vegetarian Study: an overview. Am J Clin Nutr, 1999. 70(3 Suppl): p. 525S-531S.

9. Davey, G.K., et al., EPIC-Oxford: lifestyle characteristics and nutrient intakes in a cohort of 33883 meat-eaters and 31546 non meat-eaters in the UK. Public Health Nutr, 2003. 6(3): p. 259-69.

10. Burr, M.L. and P.M. Sweetnam, Vegetarianism, dietary fiber, and mortality. Am J Clin Nutr, 1982. 36(5): p. 873-7.

11. Key, T.J., et al., Mortality in vegetarians and nonvegetarians: detailed findings from a collaborative analysis of 5 prospective studies. Am J Clin Nutr, 1999. 70(3 Suppl): p. 516S-524S.

12. Tonstad, S., et al., Vegetarian diets and incidence of diabetes in the Adventist Health Study-2. Nutr Metab Cardiovasc Dis, 2011.

13. Rizzo, N.S., et al., Vegetarian dietary patterns are associated with a lower risk of metabolic syndrome: the adventist health study 2. Diabetes Care, 2011. 34(5): p. 1225-7.

14. Key, T.J., et al., Cancer incidence in British vegetarians. Br J Cancer, 2009. 101(1): p. 192-7.

15. Fraser, G.E., Diet, Life Expectancy, and Chronic Disease. Studies of Seventh-day Adventists and Other Vegetarians. 2003, New York: Oxford University Press.

16. Key, T. and G. Davey, Prevalence of obesity is low in people who do not eat meat. Bmj, 1996. 313(7060): p. 816-7.

17. Ferdowsian, H.R. and N.D. Barnard, Effects of plant-based diets on plasma lipids. Am J Cardiol, 2009. 104(7): p. 947-56.

18. Pettersen B.J., et al., Vegetarian diets and blood pressure among white subjects: results from the Adventist Health Study-2 (AHS-2). Public Health Nutr, 2012: p. 1-8.

19. Vinnari, M., et al., Identifying vegetarians and their food consumption according to self-identification and operationalized definition in Finland. Public Health Nutr, 2009. 12(4): p. 481-8.

20. Haddad, E.H. and J.S. Tanzman, What do vegetarians in the United States eat? Am J Clin Nutr, 2003. 78(3 Suppl): p. 626S-632S.

21. van den Brandt, P.A., et al., A large-scale prospective cohort study on diet and cancer in The Netherlands. J Clin Epidemiol, 1990. 43(3): p. 285-95.

22. Goldbohm, R.A., et al., Validation of a dietary questionnaire used in a large-scale prospective cohort study on diet and cancer. Eur J Clin Nutr, 1994. 48(4): p. 253-65.

23. Willett, W.C., Implications of total energy intake for epidemiologic studies of breast and large-bowel cancer. Am J Clin Nutr, 1987. 45(1 Suppl): p. 354-60.

24. Fraser, G.E., Vegetarian diets: what do we know of their effects on common chronic diseases? Am J Clin Nutr, 2009. 89(5): p. 1607S-1612S.

25. de Bakker, E., Dagevos, H, Reducing Meat Consumption in Today's Consumer Society: Questioning the Citizen-Consumer Gap. J Agric Environ Ethics, 2011.

26. Ginsberg, C. The market for vegetarian foods. Vegetarian Resource Group. www.vrg.org/nutshell/market.htm 31/07/12].

27. Gilsing, A.M., et al., Serum concentrations of vitamin B12 and folate in British male omnivores, vegetarians and vegans: results from a cross-sectional analysis of the EPIC-Oxford cohort study. Eur J Clin Nutr, 2010. 64(9): p. 933-9.

28. Craig, W.J. and A.R. Mangels, Position of the American Dietetic Association: vegetarian diets. J Am Diet Assoc, 2009. 109(7): p. 1266-82.

29. Commission Directive 2008/100/EC of 28 October 2008 amending Council Directive 90/496/EEC on nutrition labelling for foodstuffs as regards recommended daily allowances, energy conversion factors and definitions Official Journal of the European Union, 2008. 51: p. L 285-259. 
30. Hurrell, R. and I. Egli, Iron bioavailability and dietary reference values. Am J Clin Nutr. 91(5): p. 1461S1467S.

31. Frentzel-Beyme, R. and J. Chang-Claude, Vegetarian diets and colon cancer: the German experience. Am J Clin Nutr, 1994. 59(5 Suppl): p. 1143S-1152S.

32. Patterson, R.E., et al., Changes in diet, physical activity, and supplement use among adults diagnosed with cancer. J Am Diet Assoc, 2003. 103(3): p. 323-8.

33. Pekmezi, D.W. and W. Demark-Wahnefried, Updated evidence in support of diet and exercise interventions in cancer survivors. Acta Oncol, 2011. 50(2): p. 167-78.

34. Snowdon, D.A., Animal product consumption and mortality because of all causes combined, coronary heart disease, stroke, diabetes, and cancer in Seventh-day Adventists. Am J Clin Nutr, 1988. 48(3 Suppl): p. 739-48.

35. Kuzma, J.W. and K.D. Lindsted, Determinants of eight-year diet recall ability. Epidemiology, 1990. 1(5): p. 386-91. 


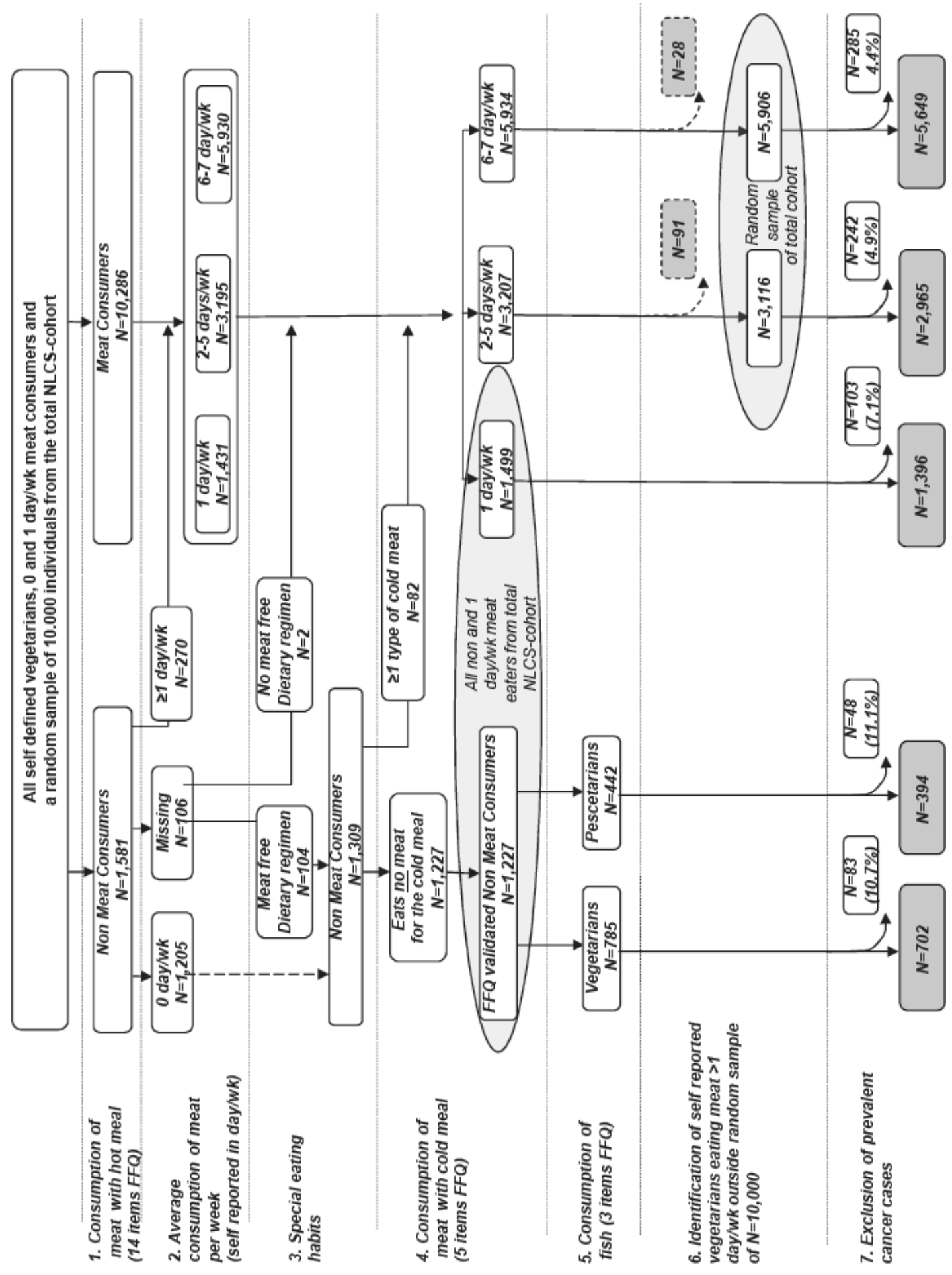





\section{CHAPTER 5}

\section{Colorectal cancer risk in vegetarians and low meat consumers: a prospective investigation}

Anne MJ Gilsing

Leo J Schouten R Alexandra Goldbohm

Pieter C Dagnelie Piet A van den Brandt and Matty P Weijenberg

Adapted version accepted for publication in Open Access Epidemiology 


\section{ABSTRACT}

Background: To study how a vegetarian or low meat diet influences the risk of colorectal cancer compared to a more common high meat diet, and to assess the explanatory role of (non-) dietary factors associated with these diets.

Methods: In the Netherlands Cohort Study - Meat Investigation Cohort (NLCS-MIC) (analytical cohort of 11082 individuals including 1040 self-defined vegetarians), subjects completed a baseline questionnaire on dietary habits and other risk factors for cancer in 1986 , based on which they were further classified into vegetarians $(n=691)$, pescetarians ( $n=389), 1$ day/week- $(n=1388), 2-5$ day/week- $(n=2965)$, and 6-7 day/week meat consumers ( $n=5649$ ). After 20.3 years of follow-up, 437 colorectal cancer cases (307 colon, 92 rectal) were available for analyses.

Results: A non-significantly decreased risk of CRC for vegetarians, pescetarians, and 1 day/week meat eaters compared to 6-7 day/week meat consumers was observed (age/sex adjusted Hazard Ratios (HR): 0.73(0.47-1.13), 0.80(0.47-1.39), and 0.72(0.52-1.00), respectively). Most of the differences in HR between these meat consumption groups could be explained by intake of dietary fiber. Other (non-)dietary factors characteristic for a vegetarian or low meat diet had negligible individual effects, but attenuated the HRs towards the null when combined. No statistically significant differences in CRC risk between vegetarians and non-vegetarians was observed, but the association was stronger using confirmed vegetarian status over self-defined status (age/sex adjusted HR: 0.78(0.51-1.20), and $0.91(0.66-1.25)$ respectively).

Conclusion: Vegetarians, pescetarians, and 1 day/week meat eaters showed a nonsignificantly decreased risk of colorectal cancer compared to 6-7 day/week meat consumers, mainly due to differences in dietary pattern other than meat intake. 


\section{INTRODUCTION}

Only a few prospective cohort studies specifically set out to study colorectal cancer incidence in vegetarians. The Oxford Vegetarian study, the EPIC-Oxford study, and the Adventists Health Study I and II, intentionally included a large proportion of vegetarians, but yielded inconsistent and mixed results [1-5].

Some of the inconsistency in findings may be owing to differences in sampling strategies between studies: some studies stem from convenience samples $[1,2,5]$ that have likely also recruited more health-conscious non-vegetarians. Other inconsistencies may arise as a result of differences in definition and operationalization of vegetarianism, and the usefulness and reliability of self-defined vegetarianism in etiological studies of cancer remains unclear. Several additional questions concerning the effect of vegetarian diets on colorectal cancer stay unanswered and require further investigation. Although it is speculated that risk factors for colon and rectal cancer may vary [6, 7], anatomic subsitespecific effects of vegetarian diets have not been reported. Moreover, it is unclear whether the association between vegetarianism and colorectal cancer risk differs by time of adherence to the dietary regimen.

Within the existing studies of meat, vegetarianism and colorectal cancer risk, little to no attention has been paid to the lower end of the meat consumption spectrum. It would be interesting to address whether complete abstinence of meat is associated with a lower colorectal cancer risk than very low meat consumption, or vice versa. Moreover, it is likely that possible beneficial effects of vegetarianism and low meat consumption can, apart from the (near) abstinence of meat, also be attributed to other dietary and lifestyle factors that characterize these diets $[8,9]$. Yet, little is known about the potential explanatory role of these multiple connected factors in the associations of vegetarian and low meat diets with colorectal cancer risk.

We investigated the association between vegetarianism, (low) meat consumption and colorectal cancer risk with special focus on colorectal sub-locations, the time of adherence to the dietary regimen, the reliability of self-defined vegetarianism, and the contribution of individual dietary and lifestyle factors within the "Netherlands Cohort Study-Meat Investigation Cohort" (NLCS-MIC). This population based cohort includes a considerable number of vegetarians, pescetarians and low meat consumers resulting in a wide distribution of dietary and lifestyle characteristics that should facilitate the identification of associations with colorectal cancer risk.

\section{METHODS}

\section{Study population and cancer follow-up}

The NLCS 'Meat Investigation Cohort' (NLCS-MIC) is an analytical cohort embedded within the ongoing prospective Netherlands Cohort Study (NLCS). The total NLCS study was initiated in September 1986 and includes 120852 men and women aged 55-69 years at baseline, largely originating from 204 municipalities with computerized population registries. In addition, to increase contrast within the cohort, vegetarians were overrepresented by recruitment through health food shops and magazines. At the start of 
the study, participants completed a self-administered questionnaire on dietary habits, lifestyle characteristics, medical history, and other potential risk factors for cancer [10]. NLCS-MIC is specifically established within the NLCS to study the health effects of vegetarian and low meat diets. Because the total NLCS-cohort traditionally uses the casecohort approach for analyses, data was only entered for a random subcohort of 10000 subjects and all enumerating cancer cases. As a result, NLCS-MIC had to be established by combining the random subcohort with all (self-reported) vegetarians and all individuals that consumed meat only 1 day/week from the total NLCS cohort for whom the data was also entered. All vegetarians and those consuming meat 1 day/week were initially identified based on two items relating to specific dietary regimens that are stated on the first page of the questionnaire that was processed for all 120,852 cohort members: "Do you have any special eating habits?", and "how many days on average per week do you eat meat?". The 150 item semi-quantitative food frequency questionnaire (FFQ) was used to accurately categorize NLCS-MIC ( $n=11082)$ with complete and consistent FFQs into five meat consumption categories: confirmed vegetarians $(n=691)$ and pescetarians $(n=389), 1$ day/week- $(n=1,388), 2-5$ days/week- $(n=2965)$, and 6-7 days/week meat consumers $(n=5649)$. We defined vegetarians as individuals who consume a diet void of meat (including vegans, lactoovo-, lacto-, and ovo-vegetarians). Pescetarians do not eat meat but do eat fish. As a consequence of the procedure followed, NLCS-MIC also includes 1133 selfreported vegetarians of whom 109 reported to consume meat but were not part of the randomly selected subcohort. As a result, these latter individuals are only included in analyses when comparing all self-reported vegetarians (either confirmed or not) to the complementary group of non-(self-reported) vegetarians, and not for all other contrasts. Full details of the study design have been described elsewhere [8].

The full-cohort approach is used for analyses of NLCS-MIC. NLCS-MIC is being monitored for cancer occurrence by repeated record linkage to the Netherlands Cancer Registry, the Dutch Pathology Registry, and the cause of death registry (Statistics Netherlands), together providing a near $100 \%$ coverage [11]. Follow-up for vital status was established by record linkage to the automated municipal population registries and the Central Bureau for Genealogy. Less than $1 \%$ of the cohort members were lost to follow-up. After 20.3 years of follow-up and exclusion of prevalent cancer cases at baseline (other than skin cancer), 477 colorectal cancer cases (336 colon (ICD-O codes: 153.0-153.7) (184 proximal colon; 142 distal colon and 10 unspecified) and 99 rectum (ICD-O code: 154.1)) remained eligible for analyses. Recto sigmoid cancer cases (ICD-O code: 154.0) were not evaluated separately because of the small number of cases $(n=42)$ and the higher risk of misclassification [12]. The NLCS has been approved by the institutional review boards of the TNO Quality of Life Research Institute (Zeist, the Netherlands) and Maastricht University (Maastricht, the Netherlands).

\section{Questionnaire}

All participants completed a 150 item semi-quantitative FFQ at baseline, estimating the average frequency and amount of foods and beverages consumed over the previous 12 months. Next to the questions relating to special eating habits and weekly meat consumption frequency that were used for the identification of vegetarians and 1 day/week meat consumers, the questionnaire also assessed the time since the start of any special eating habits and weekly frequency of meat consumption (for 0-1 day/week meat 
consumers), in years until baseline (1986). In addition, the FFQ contained 14 items on the consumption of meat with the hot meal (mainly fresh meat, including chicken), 5 items on the consumption of meat products used as sandwich fillings, and 3 items on fish consumption (with the hot meal, for lunch, as a snack in between meals). Coding of fresh meat items was based on raw weight to take into account the amount of fat originally present in the meat but eventually ending up in the gravy, which is usually consumed as well. Processed meat was defined as meat items that had undergone some form of preservation (mostly cured (i.e. treated with nitrite/nitrate salt, sometimes smoked and/or fermented)).

A validation study conducted in a subgroup of the cohort two years after the baseline measurement indicated that the Spearman correlation coefficients for meat, meat products and fish as assessed by the questionnaire and those estimated from the 9-day record were $0.46,0.54$ and 0.53 respectively. The number of vegetarians and 1 day/week meat consumers was too low in this validation sample to assess a correlation in these extremes [13].

\section{Statistical analyses}

We estimated the association between meat consumption group (confirmed vegetarian, pescetarian, 1 day/week-, 2-5 days/week-, and 6-7 days/week meat consumers (reference group)) and the risk of colorectal cancer. In addition, the association with self-reported vegetarian status (self-reported versus complementary group of non-self-reportedvegetarians) and confirmed vegetarian status (confirmed vegetarian versus complementary group of non-vegetarians) was examined. To increase power, vegetarians and fish eaters were combined in an overlapping category of non-meat consumers to examine whether their risk of colorectal cancer differed from the complementary group of individuals who do consume meat. To assess to what extent these associations can (partially) be explained by other dietary and lifestyle variables (e.g. smoking, physical activity, BMI and level of education), we calculated the difference in risk estimate, firstly adjusting for age and sex, then further adjusting for energy and each food group, or lifestyle factor in turn. Moreover, the association with meat consumption group and confirmed vegetarians status was stratified by duration of adherence to the specific diet ( $\leq 10$ years, $>10$ years).

Individuals adhering to a no or low meat diet often replace the meat in their diet with other protein-rich food groups. Using nutrient density substitution models and meat protein as a proxy for meat intake, we examined the effect of replacing one protein subtype for another by including nutrient density variables for all but one protein subtype in a multivariable model along with total protein intake and total energy intake [14].

The following food groups and foods were also selected for analyses (in g/day): fresh meat (beef, pork, minced meat, chicken, liver), processed meat, fish, fresh red meat (fresh meat without chicken) and beef, pork, minced meat, chicken, and liver as separate types. For the individual meat types, subjects were classified into non-consumers, and tertiles of consumers (highest tertile as reference group), and as continuous variables. The latter were reported in $50 \mathrm{~g} /$ day increase for all fresh meat types except liver, and $25 \mathrm{~g} /$ day for processed meat and liver intake. For some variables, categories were used instead of quintiles. For liver intake, there was a non-user and a user group ( $>0 \mathrm{~g} /$ day). For both 
chicken and fish a non-user and 3 user categories $(0,0-<6.6, \geq 6.6-<22.8$ and $\geq 22.8 \mathrm{~g} /$ day for chicken; $0,0-<10, \geq 10-<20$ and $\geq 20 \mathrm{~g} /$ day for fish) were defined.

For all the above described contrasts, age and sex adjusted and multi-variable adjusted hazard rate ratios (HRs) and their corresponding 95\% confidence intervals $(95 \% \mathrm{Cl})$ were estimated using Cox proportional hazards models. The proportional hazards assumption was tested using the scaled Schoenfeld residuals. To evaluate whether early symptoms of colorectal cancer before diagnosis could have influenced the results, early cases (diagnosed within 2 years after baseline) were excluded in additional analyses. The covariates included in the multivariate analyses were either a priori selected risk factors of colorectal cancer, or variables that changed the risk estimates for meat consumption group, vegetarian status or total fresh meat intake by $10 \%$ or more. The latter criterion was not met for any other than the predefined covariates resulting in a final model including age(years), sex, total energy intake(kcal/day), cigarette smoking (never, ever, current), alcohol consumption (g/day), BMI $\left(\mathrm{kg} / \mathrm{m}^{2}\right)$, non-occupational physical activity $(\leq 30,>30-\leq 60,>60-\leq 90,>90$ minutes/day), and level of education (lower vocational, secondary-/medium-vocational, university and higher vocational). The independent contribution of the individual meat categories was examined by constructing addition models that summed to total meat.

To enable comparison, the age and sex-adjusted analyses were restricted to subjects included in multivariable-adjusted, leaving 10,210 cohort members, including 437 colorectal cancer cases (307 colon (129 proximal colon; 169 distal colon and 9 unspecified) and 92 rectal) for analyses. Moreover, when analyzing the contrast between self-reported vegetarians and non-vegetarians, an additional 90 self-reported vegetarians that reported to consume meat but were not part of the randomly selected subcohort were also included in analyses (including 7 colorectal cancer cases). Linear trends were evaluated with the Wald test by entering the categorical exposure variables as a continuous term in the Cox regression model.

To test for heterogeneity between the colon and the rectum, and the anatomic subsites of colon cancer (proximal/distal), the competing risks procedure in Stata was used. However, the standard error for the difference of the log-HRs from this procedure assumes independence of both estimated HRs which would overestimate the standard error and thus overestimate the $P$ values for their difference. Therefore, these $P$ values and the associated confidence intervals were estimated based on a bootstrapping method. Each bootstrap analysis was based on 1,000 replications.

All tests were two-tailed and differences were regarded as statistically significant at $P<0.05$. All analyses were performed using STATA Statistical Software (Intercooled STATA, version 12; Stata-Corp LP, College Station, TX).

\section{RESULTS}

The distribution of demographic and (non-) dietary characteristics according to meat consumption group has been described previously [8, Chapter 4]. The percentage of men and supplement users was higher in rectal cancer cases than in non-cases $(P<0.05)($ Table 1). 
Table 1: Baseline characteristics (means or percent) and dietary intakes of exposures of interest of colorectal cancer cases and non-cases in NLCS-MIC, 1986-2006.

\begin{tabular}{|c|c|c|c|c|}
\hline Characteristics & $\begin{array}{l}\text { Non } \\
\text { cases }\end{array}$ & $\begin{array}{l}\text { Colorectal cancer } \\
\text { cases }\end{array}$ & $\begin{array}{l}\text { Colon cancer } \\
\text { cases }^{\text {a }}\end{array}$ & $\begin{array}{l}\text { Rectal cancer } \\
\text { cases }^{\text {a }}\end{array}$ \\
\hline $\mathrm{N}$ & 9773 & 437 & 307 & 92 \\
\hline \multicolumn{5}{|l|}{ Diet Group (\%) } \\
\hline Vegetarian & $6 \%$ & $5 \%$ & $6 \%$ & $1 \%$ \\
\hline Pescetarian & $4 \%$ & $3 \%$ & $4 \%$ & $2 \%$ \\
\hline 1 day/wk meat & $12 \%$ & $9 \%$ & $10 \%$ & $11 \%$ \\
\hline 2-5 days/wk meat & $26 \%$ & $27 \%$ & $27 \%$ & $25 \%$ \\
\hline 6-7 days/wk meat & $51 \%$ & $56 \%$ & $53 \%$ & $61 \%$ \\
\hline Sex (\% men) & $47 \%$ & $53 \% *$ & $50 \%$ & $64 \% *$ \\
\hline Age $(y)$ & $61.3 \pm 4.2^{b}$ & $61.4 \pm 4.0$ & $61.5 \pm 4.1$ & $61.2 \pm 3.8$ \\
\hline Current smokers (\%) & $26 \%$ & $23 \%$ & $21 \%$ & $33 \%$ \\
\hline BMI mean & $24.7 \pm 3.2$ & $25.0 \pm 3.2$ & $24.9 \pm 3.2$ & $25.1 \pm 3.1$ \\
\hline \multicolumn{5}{|c|}{ Physical activity (non-occupational) (\%) } \\
\hline$<30 \mathrm{~min} /$ day & $21 \%$ & $19 \%$ & $19 \%$ & $15 \%$ \\
\hline $30-60 \mathrm{~min} /$ day & $30 \%$ & $33 \%$ & $35 \%$ & $30 \%$ \\
\hline $60-90 \mathrm{~min} / \mathrm{day}$ & $23 \%$ & $19 \%$ & $21 \%$ & $16 \%$ \\
\hline$>90 \mathrm{~min} /$ day & $26 \%$ & $28 \%$ & $25 \%$ & $38 \%$ \\
\hline \multicolumn{5}{|l|}{ Level of education (\%) } \\
\hline Low & $46 \%$ & $47 \%$ & $45 \%$ & $51 \%$ \\
\hline Medium & $37 \%$ & $37 \%$ & $38 \%$ & $37 \%$ \\
\hline High & $17 \%$ & $16 \%$ & $17 \%$ & $12 \%$ \\
\hline Supplement use (\% users) & $34 \%$ & $31 \%$ & $36 \%$ & $22 \% *$ \\
\hline Energy (kcal) & $1888 \pm 519$ & $1919 \pm 512$ & $1888 \pm 515$ & $2032 \pm 503 *$ \\
\hline Fiber (g) & $28.2 \pm 7.6$ & $27.8 \pm 7.2$ & $27.8 \pm 7.3$ & $27.9 \pm 6.5$ \\
\hline Alcohol (g) & $9.3 \pm 13.8$ & $11.8 \pm 16.4 *$ & $11.9 \pm 16.7 *$ & $12.3 \pm 17.2 *$ \\
\hline Total fresh meat $(\mathrm{g})^{\mathrm{c}}$ & $81.1 \pm 52.8$ & $83.6 \pm 48.1$ & $80.9 \pm 48.6$ & $88.2 \pm 48.4$ \\
\hline Fresh red meat $(\mathrm{g})^{d}$ & $70.6 \pm 49.2$ & $73.1 \pm 45.5$ & $70.7 \pm 46.0$ & $76.5 \pm 44.3$ \\
\hline $\operatorname{Beef}(\mathrm{g})$ & $21.6 \pm 23.9$ & $22.1 \pm 21.3$ & $22.4 \pm 21.9$ & $19.5 \pm 19.0$ \\
\hline Pork (g) & $30.5 \pm 29.8$ & $32.4 \pm 29.0$ & $29.9 \pm 27.6$ & $36.7 \pm 28.6 *$ \\
\hline Minced meat (g) & $14.9 \pm 16.3$ & $15.3 \pm 15.0$ & $15.1 \pm 14.8$ & $16.7 \pm 16.7$ \\
\hline Liver (g) & $1.6 \pm 4.0$ & $1.6 \pm 3.7$ & $1.5 \pm 3.6$ & $1.9 \pm 4.1$ \\
\hline Chicken (g) & $11.3 \pm 14.8$ & $11.2 \pm 14.1$ & $10.9 \pm 12.3$ & $12.3 \pm 15.1$ \\
\hline Processed meat (g) & $11.0 \pm 14.3$ & $12.3 \pm 14.4$ & $11.4 \pm 13.6$ & $16.3 \pm 17.8 *$ \\
\hline Fish (g) & $13.1 \pm 18.1$ & $12.6 \pm 14.9$ & $13.3 \pm 15.8$ & $13.3 \pm 12.9$ \\
\hline Vegetables (g) & $199 \pm 89$ & $200 \pm 85$ & $200 \pm 88$ & $204 \pm 81$ \\
\hline Fruits (g) & $184 \pm 128$ & $175 \pm 115$ & $176 \pm 115$ & $168 \pm 110$ \\
\hline Pulses (g) & $10.0 \pm 16.7$ & $10.1 \pm 17.1$ & $9.9 \pm 13.3$ & $9.5 \pm 13.9$ \\
\hline Soya products (g) & $3.1 \pm 17.8$ & $2.0 \pm 9.4$ & $2.7 \pm 9.9$ & $0.4 \pm 1.5$ \\
\hline Milk (g) & $312 \pm 208$ & $285 \pm 189 *$ & $288 \pm 193 *$ & $284 \pm 188$ \\
\hline Cheese (g) & $25.9 \pm 22.6$ & $24.2 \pm 20.8$ & $23.1 \pm 20.0 *$ & $30.4 \pm 24.4$ \\
\hline Eggs (g) & $15.6 \pm 11.7$ & $16.2 \pm 11.1$ & $15.3 \pm 10.0$ & $18.7 \pm 14.7 *$ \\
\hline
\end{tabular}

$\overline{{ }^{a} \text { Colon and rectal cases do not sum to total colorectal cancer cases because rectosigmoid cases were not evaluated }}$ separately.

${ }^{\mathrm{b}}$ Mean $\pm \mathrm{SD}$, all such values.

${ }^{\mathrm{c}}$ Intake based on raw meat weight.

${ }^{\mathrm{d}}$ Includes beef, pork, minced meat, liver and other meat.

* Statistically significant different from non-cases (using the $\chi 2$ test for categorical variables and ANOVA for continuous variables). 
The percentage of vegetarians was lowest in rectal cancer cases while the proportion of 6-7 day/week meat consumers was highest in this group. The latter is also reflected in a higher total energy intake, and consumption of pork and processed meat among the rectal cancer cases compared to non-cases $(P<0.05)$. However, non-cases had higher daily intakes of milk and cheese, but lower intakes of alcohol than cases $(P<0.05)$. Rectum cancer cases consumed more eggs than non-cases.

Table 2A and Table 2B show hazard rate ratios and 95\% confidence intervals for colorectal and colon and rectal cancer, respectively, according to meat consumption group, vegetarian status and meat consumption status, adjusted for age and sex alone and with further adjustment for confounders. There was no evidence of an interaction by sex for any of the comparisons made ( $P$-interaction>0.05). A borderline statistically significant reduced risk of colorectal cancer for 1 day/week meat consumers was found when compared to 6-7 days/week meat consumers ( $\mathrm{HR}=0.72,95 \% \mathrm{Cl}$ : 0.52-1.00).

Table 2A: Hazard rate ratios (HR) and 95\% confidence intervals (CI) for colorectal cancer according to meat consumption group, vegetarian status and meat consumption status.

\begin{tabular}{|c|c|c|c|c|c|c|c|}
\hline \multirow[b]{2}{*}{ Factor } & \multirow[b]{2}{*}{ Category } & \multirow[b]{2}{*}{ PY } & \multicolumn{5}{|c|}{ Colorectal cancer } \\
\hline & & & Cases & $\mathrm{HR}$ & $(95 \% \mathrm{Cl})^{\mathrm{a}}$ & $\mathrm{HR}$ & $(95 \% \mathrm{Cl})^{\mathrm{b}}$ \\
\hline \multicolumn{8}{|l|}{ Meat consumption } \\
\hline \multirow[t]{6}{*}{ group } & vegetarian $^{c}$ & 11277 & 22 & 0.73 & $(0.47-1.13)$ & 0.83 & $(0.53-1.31)$ \\
\hline & pescetarian & 6429 & 14 & 0.80 & $(0.47-1.39)$ & 0.88 & $(0.51-1.51)$ \\
\hline & $1 \mathrm{day} /$ wk meat & 21451 & 41 & 0.72 & $(0.52-1.00)$ & 0.77 & $(0.55-1.08)$ \\
\hline & 2-5 day/wk meat & 43360 & 116 & 0.93 & $(0.75-1.16)$ & 0.95 & $(0.76-1.19)$ \\
\hline & 6-7 day/wk meat & 87356 & 244 & 1 (ref) & & 1 (ref) & \\
\hline & $P_{\text {trend }}$ & & & 0.04 & & 0.19 & \\
\hline \multirow[t]{4}{*}{ Vegetarianism } & vegetarian $^{c}$ & 11277 & 22 & 0.78 & $(0.51-1.20)$ & 0.89 & $(0.58-1.39)$ \\
\hline & non vegetarian & 160598 & 415 & 1 (ref) & & 1 (ref) & \\
\hline & self-defined vegetarian ${ }^{d}$ & 18272 & 42 & 0.91 & $(0.66-1.25)$ & 1.04 & $(0.74-1.44)$ \\
\hline & non self-defined vegetarian & 154961 & 402 & 1 (ref) & & 1 (ref) & \\
\hline \multirow[t]{4}{*}{ Meat consumption } & No meat consumers & 17706 & 36 & 0.81 & $(0.57-1.14)$ & 0.90 & $(0.63-1.29)$ \\
\hline & Meat consumers & 154169 & 401 & 1 (ref) & & 1 (ref) & \\
\hline & $\leq 1$ day/wk meat & 39158 & 77 & 0.75 & $(0.58-0.97)$ & 0.82 & $(0.63-1.06)$ \\
\hline & $>1$ day/wk meat & 132717 & 360 & 1 (ref) & & 1 (ref) & \\
\hline \multicolumn{8}{|c|}{ PY (person years at risk). } \\
\hline \multicolumn{8}{|c|}{ adjusted for age (y) and sex. } \\
\hline \multicolumn{8}{|c|}{$\begin{array}{l}{ }^{b} \text { Adjusted for age }(\mathrm{y}) \text {, sex, total energy intake (kcal), cigarette smoking (never, ever, current), alcohol } \\
\text { consumption (g/day), BMI }\left(\mathrm{kg} / \mathrm{m}^{2}\right) \text {, non-occupational physical activity }(<30,30-60,60-90,>90 \mathrm{~min} / \mathrm{d}) \text {, and level } \\
\text { of education (lower vocational, second and medium vocational, university and higher vocational). }\end{array}$} \\
\hline \multicolumn{8}{|c|}{$\begin{array}{l}{ }^{c} \text { Confirmed vegetarians based on the extensive FFQ (defined as individuals who consume a diet void of meat } \\
\text { and fish). }\end{array}$} \\
\hline \multicolumn{8}{|c|}{$\begin{array}{l}\text { NLCS-MIC includes } 1,133 \text { self-defined vegetarians of whom } 109 \text { reported to consume meat but were not part } \\
\text { of the randomly selected subcohort. These individuals are only included in analyses when comparing all self- } \\
\text { defined vegetarians (either FFQ confirmed or not) to non-self-defined vegetarians, and not for all other } \\
\text { contrasts. }\end{array}$} \\
\hline
\end{tabular}




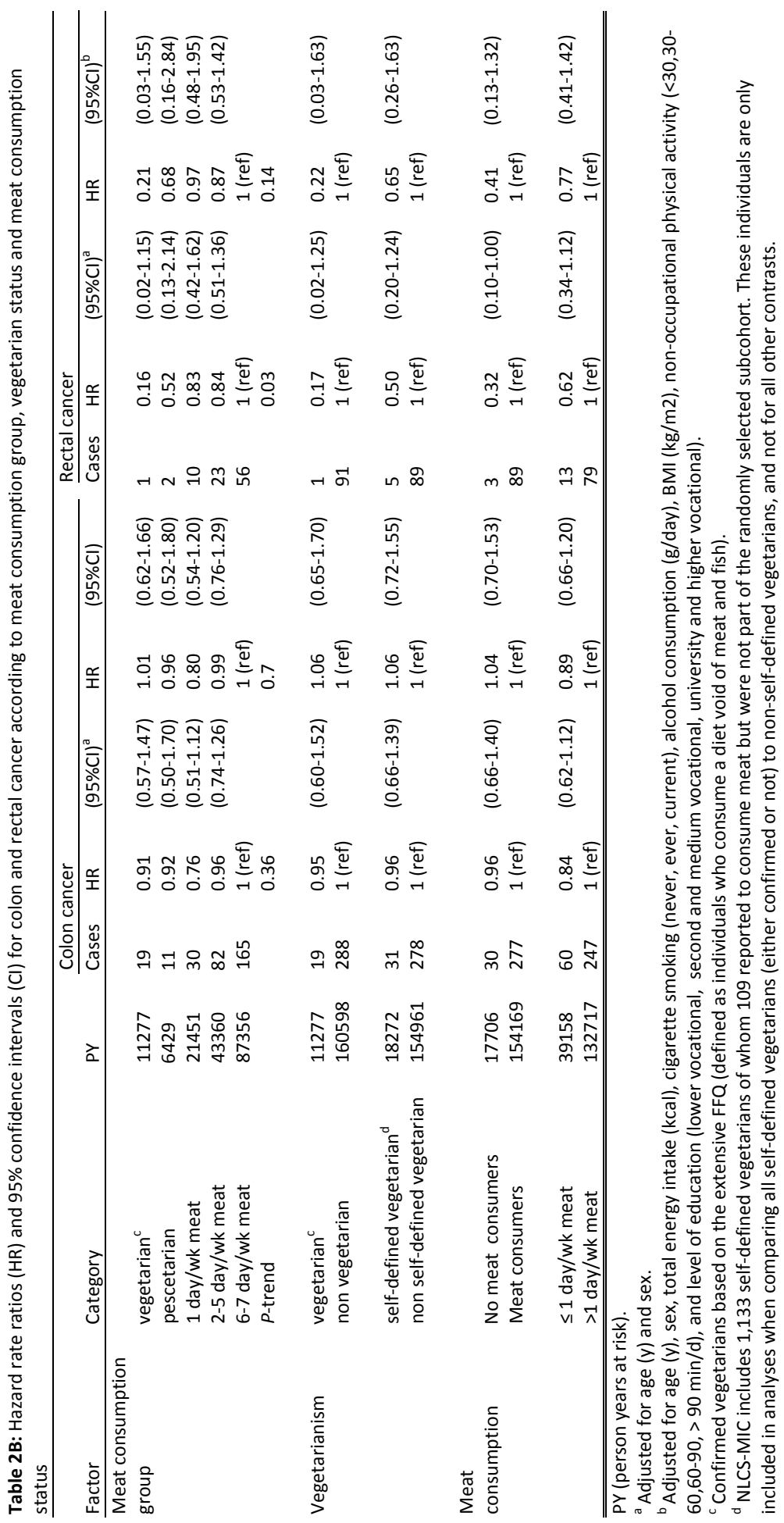


Further adjustment for confounding attenuated this association $(\mathrm{HR}=0.77,95 \% \mathrm{Cl}$ : $0.55-$ 1.09). A similar though not statistically significant pattern was observed for colon cancer, while the HR for rectal cancer was lowest among the vegetarians $(\mathrm{HR}=0.21,95 \% \mathrm{Cl}: 0.03-$ 1.51 ) and increased with increasing frequency of meat intake ( $P$-trend $=0.03$ (age-/energyadjusted model)). There was only one rectal cancer case that adhered to a vegetarian diet. The inverse association between risk of colorectal cancer and confirmed or self-reported vegetarian status did not reach statistical significance, but was notably attenuated using self-definition ( $\mathrm{HR}=0.78,95 \% \mathrm{Cl}$ : $0.51-1.20$ and $\mathrm{HR}=0.91,95 \% \mathrm{Cl}$ : $0.66-1.25$, respectively). Similar findings were observed for rectal cancer, whereas HRs around 1 were observed for colon cancer risk. Although the effect of the five meat consumption groups did not differ between colon and rectal cancer cases, the effect of vegetarian status (confirmed versus complementary group of non-confirmed vegetarians) was significantly different between both endpoints $(P$-heterogeneity $=0.02)$. When vegetarians, pescetarians and 1 day/week meat consumers were combined and compared against individuals eating meat $>1$ day/week, a statistically significant $25 \%$ reduction in risk of colorectal cancer was observed, which was no longer significant after adjustment for confounders.

Within the colon, the risks for proximal or distal tumors were not statistically different for either meat consumption group $(P$-heterogeneity $=0.26)$ or confirmed vegetarian status $(P$ heterogeneity=0.06). Estimates for distal colon cancer were most comparable to those observed for rectal cancer (Supplemental Table 1). Moreover, in a lag analysis excluding the first 2 years of follow-up (408 colorectal cancer cases), the findings for meat consumption group and vegetarian status did not change appreciably (data not shown).

Table 3 shows the percent change in HR for overall colorectal cancer risk across the meat consumption groups, firstly adjusted for age and sex, then further adjusted for energy and each food group, or lifestyle factor in turn. Fiber intake contributed most to the observed inverse risk of colorectal cancer when comparing vegetarians, pescetarians, and 1 day-week meat eaters to 6-7 day-week meat. When all confounding dietary and lifestyle factors were added together in one model simultaneously, the HRs approached the null. Comparable patterns were observed when examining colon and rectal cancer separately.

In addition, we observed evidence that substituting 5\% of energy from protein from meat by $5 \%$ of energy from dairy protein was associated with a $24 \%$ reduced risk of colorectal cancer, after adjustment for confounding variables $(P=0.055)$ (Supplemental Table 2). Substituting protein from meat by other sources of protein (e.g. protein from plant sources, eggs or fish) had no statistically significant effect on risk for colorectal cancer.

Supplemental Table 3 shows the HRs for colorectal cancer according to meat consumption group and vegetarian status stratified by time of adherence to the vegetarian and 1 day/week meat diet ( $\leq 10$ years versus $>10$ years). The HRs were comparable to the overall effects presented in Table 2 but none of the comparisons reached statistical significance. No striking differences between short term and long term adherence to a vegetarian or low meat diet were observed regarding colorectal cancer risk. 
Table 3: Difference in Hazard rate ratios (HR) for colorectal cancer between vegetarians, pescetarians, 1 day/wk meat and 6-7 days/wk meat consumers after adjustment for individual dietary and lifestyle factors.

\begin{tabular}{|c|c|c|c|c|c|c|c|}
\hline \multirow[b]{4}{*}{ Factor adjusted for ${ }^{a}$} & \multicolumn{7}{|c|}{ Colorectal cancer } \\
\hline & \multicolumn{2}{|c|}{ Vegetarian } & \multicolumn{2}{|c|}{ Pescetarian } & \multicolumn{2}{|c|}{$\begin{array}{l}1 \text { day/ } \\
\text { wk meat }\end{array}$} & \multirow[t]{3}{*}{$\begin{array}{l}\text { 6-7 day/ } \\
\text { wk meat } \\
\end{array}$} \\
\hline & & $\%$ change & & $\begin{array}{l}\% \\
\text { change }\end{array}$ & & $\%$ change & \\
\hline & $\mathrm{HR}$ & in $\mathrm{HR}$ & $\mathrm{HR}$ & in $\mathrm{HR}$ & $\mathrm{HR}$ & in $\mathrm{HR}$ & \\
\hline Age and sex & 0.73 & - & 0.80 & - & 0.72 & - & 1 (ref) \\
\hline \multicolumn{8}{|l|}{ Dietary Factors } \\
\hline Energy (kcal) & 0.73 & $0 \%$ & 0.80 & $0 \%$ & 0.71 & $-1 \%$ & 1 (ref) \\
\hline Energy + alcohol (g) & 0.77 & $5 \%$ & 0.82 & $2 \%$ & 0.74 & $3 \%$ & 1 (ref) \\
\hline Energy + fiber $(g)$ & 0.82 & $12 \%$ & 0.89 & $11 \%$ & 0.76 & $6 \%$ & 1 (ref) \\
\hline Energy + fruits (g) & 0.75 & $3 \%$ & 0.82 & $2 \%$ & 0.72 & $0 \%$ & 1 (ref) \\
\hline Energy + vegetables (g) & 0.72 & $-1 \%$ & 0.79 & $-1 \%$ & 0.71 & $-1 \%$ & 1 (ref) \\
\hline Energy + pulses $(g)$ & 0.71 & $-3 \%$ & 0.78 & $-3 \%$ & 0.70 & $-3 \%$ & 1 (ref) \\
\hline Energy + soy products (g) & 0.78 & $7 \%$ & 0.87 & $9 \%$ & 0.73 & $1 \%$ & 1 (ref) \\
\hline Energy + milk (g) & 0.76 & $4 \%$ & 0.83 & $4 \%$ & 0.74 & $3 \%$ & 1 (ref) \\
\hline Energy + cheese (g) & 0.77 & $5 \%$ & 0.84 & $5 \%$ & 0.74 & $3 \%$ & 1 (ref) \\
\hline Energy + eggs $(g)$ & 0.73 & $0 \%$ & 0.80 & $0 \%$ & 0.71 & $-1 \%$ & 1 (ref) \\
\hline Energy + Supplement use $(0,1, \geq 2)$ & 0.75 & $3 \%$ & 0.83 & $4 \%$ & 0.73 & $1 \%$ & 1 (ref) \\
\hline Full model including dietary factors ${ }^{\mathrm{b}}$ & 0.99 & $36 \%$ & 1.04 & $30 \%$ & 0.86 & $19 \%$ & 1 (ref) \\
\hline \multicolumn{8}{|l|}{ Lifestyle Factors } \\
\hline Smoking $^{c}$ & 0.72 & $-1 \%$ & 0.79 & $-1 \%$ & 0.71 & $-1 \%$ & 1 (ref) \\
\hline Non-occupational physical activity ${ }^{d}$ & 0.73 & $0 \%$ & 0.81 & $1 \%$ & 0.72 & $0 \%$ & 1 (ref) \\
\hline $\mathrm{BMI}\left(\mathrm{kg} / \mathrm{m}^{2}\right)$ & 0.77 & $5 \%$ & 0.84 & $5 \%$ & 0.74 & $3 \%$ & 1 (ref) \\
\hline Level of education ${ }^{e}$ & 0.75 & $3 \%$ & 0.83 & $4 \%$ & 0.73 & $1 \%$ & 1 (ref) \\
\hline $\begin{array}{l}\text { Full model including dietary and lifestyl } \\
\text { factors }{ }^{\mathrm{b}}\end{array}$ & 1.06 & $45 \%$ & 1.11 & $39 \%$ & 0.89 & $24 \%$ & 1 (ref) \\
\hline
\end{tabular}

No clear association was observed when the relation was examined between dietary intake of individual meat types and the risk of overall colorectal cancer (Table 4A). Similar findings were observed for colon cancer risk (Table 4B). When restricting analyses to the rectal cancer subtype, we observed HRs below 0.65 (not statistically significant) for fresh meat, fresh red meat, and pork consumers when comparing for the non-consumers to those in the highest tertile (Table 4B). A statistically significant increased risk for rectal cancer was observed with each 25 g-day increment in processed meat intake (HR: $1.36,95 \% \mathrm{Cl}$ : $1.01-$ $1.81)$ as well as a significant trend across categories of intake ( $P$-trend $=0.008)$. 
Table 4A: Hazard rate ratios (HR) and 95\% confidence intervals (CI) for colorectal cancer according to sex-specific quintiles and categories of intake of fresh meat, types of fresh meat, and processed meat.

\begin{tabular}{|c|c|c|c|c|c|c|c|c|}
\hline \multirow[b]{2}{*}{ Food item } & \multicolumn{2}{|c|}{ Median intake } & \multirow[b]{2}{*}{ PY } & \multicolumn{5}{|c|}{ Colorectal cancer } \\
\hline & Men & Women & & Cases & $\mathrm{HR}$ & $(95 \% \mathrm{Cl})^{\mathrm{a}}$ & $\mathrm{HR}$ & $(95 \% \mathrm{Cl})^{b}$ \\
\hline \multicolumn{9}{|c|}{ Total fresh meat $\left(\mathrm{g} /\right.$ day) ${ }^{\mathrm{c}}$} \\
\hline Non consumers & 0 & 0 & 20022 & 42 & 0.90 & $(0.64-1.28)$ & 1.09 & $(0.75-1.59)$ \\
\hline $\mathrm{T} 1$ & 56.6 & 30.0 & 49317 & 111 & 0.93 & $(0.72-1.20)$ & 1.04 & $(0.79-1.35)$ \\
\hline $\mathrm{T} 2$ & 99.5 & 85.7 & 51037 & 161 & 1.32 & $(1.04-1.66)$ & 1.37 & $(1.08-1.74)$ \\
\hline T3 & 142.6 & 123.8 & 51499 & 123 & 1 (ref) & & 1 (ref) & \\
\hline \multicolumn{3}{|c|}{$P_{\text {trend }}$} & & & 0.28 & & 0.88 & \\
\hline \multicolumn{4}{|c|}{ Continuous (50g/day intake increment) } & & 1.04 & $(0.94-1.13)$ & 0.97 & $(0.88-1.08)$ \\
\hline \multicolumn{9}{|c|}{ Fresh red meat $(\mathrm{g} / \text { day })^{\mathrm{c}}$} \\
\hline Non consumers & 0 & 0 & 21571 & 42 & 0.75 & $(0.53-1.06)$ & 0.83 & $(0.58-1.21)$ \\
\hline T1 & 47.3 & 24.9 & 48721 & 121 & 0.92 & $(0.72-1.18)$ & 0.99 & $(0.77-1.28)$ \\
\hline $\mathrm{T} 2$ & 85.5 & 71.2 & 50679 & 138 & 1.02 & $(0.80-1.29)$ & 1.05 & $(0.83-1.34)$ \\
\hline T3 & 127.6 & 106.9 & 20904 & 136 & 1 (ref) & & 1 (ref) & \\
\hline \multicolumn{3}{|c|}{$P_{\text {trend }}$} & & & 0.12 & & 0.48 & \\
\hline \multicolumn{4}{|c|}{ Continuous (50g/day intake increment) } & & 1.04 & $(0.95-1.15)$ & 1.00 & $(0.90-1.11)$ \\
\hline \multicolumn{9}{|c|}{$\operatorname{Beef}(g / \text { day })^{c}$} \\
\hline Non consumers & 0 & 0 & 37701 & 87 & 0.89 & $(0.68-1.18)$ & 0.96 & $(0.72-1.30)$ \\
\hline T1 & 8.6 & 6.3 & 44979 & 106 & 0.89 & $(0.68-1.15)$ & 0.92 & $(0.71-1.20)$ \\
\hline $\mathrm{T} 2$ & 24.1 & 19.3 & 45099 & 125 & 1.05 & $(0.81-1.34)$ & 1.07 & $(0.83-1.37)$ \\
\hline T3 & 48.9 & 43.2 & 44096 & 119 & 1 (ref) & & 1 (ref) & \\
\hline \multicolumn{3}{|c|}{$P_{\text {trend }}$} & & & 0.24 & & 0.58 & \\
\hline \multicolumn{4}{|c|}{ Continuous (50g/day intake increment) } & & 1.02 & $(0.84-1.25)$ & 0.96 & $(0.78-1.18)$ \\
\hline \multicolumn{9}{|c|}{ Pork (g/day) $)^{c}$} \\
\hline Non consumers & 0 & 0 & 30814 & 62 & 0.82 & $(0.61-1.12)$ & 0.88 & $(0.62-1.25)$ \\
\hline T1 & 11.1 & 6.1 & 46148 & 109 & 0.93 & $(0.72-1.21)$ & 1.01 & $(0.77-1.32)$ \\
\hline $\mathrm{T} 2$ & 35.0 & 28.5 & 47628 & 148 & 1.24 & (0.98-1.58) & 1.32 & $(1.03-1.68)$ \\
\hline T3 & 66.9 & 58.3 & 47285 & 118 & 1 (ref) & & 1 (ref) & \\
\hline \multicolumn{3}{|l|}{$P_{\text {trend }}$} & & & 0.10 & & 0.45 & \\
\hline \multicolumn{4}{|c|}{ Continuous (50g/day intake increment) } & & 1.10 & $(0.94-1.28)$ & 1.02 & $(0.86-1.21)$ \\
\hline \multicolumn{9}{|c|}{ Minced meat $(g / \text { day })^{c}$} \\
\hline Non consumers & 0 & 0 & 40870 & 81 & 0.82 & $(0.61-1.10)$ & 0.85 & $(0.62-1.16)$ \\
\hline T1 & 6.8 & 5.5 & 43368 & 112 & 1.04 & $(0.80-1.36)$ & 1.07 & $(0.82-1.41)$ \\
\hline $\mathrm{T} 2$ & 16.7 & 14.0 & 44180 & 137 & 1.26 & $(0.98-1.62)$ & 1.27 & $(0.99-1.64)$ \\
\hline $\mathrm{T} 3$ & 34.0 & 29.0 & 43456 & 107 & 1 (ref) & & 1 (ref) & \\
\hline$P_{\text {trend }}$ & & & & & 0.10 & & 0.32 & \\
\hline Continuous (50g) & day inta & e increme & & & 1.06 & $(0.80-1.40)$ & 0.97 & $(0.72-1.32)$ \\
\hline Liver $(g / \text { day })^{c}$ & & & & & & & & \\
\hline Non consumers & 0 & 0 & 120706 & 300 & 0.92 & $(0.75-1.13)$ & 0.93 & $(0.75-1.14)$ \\
\hline Consumers & 4.1 & 3.3 & 51169 & 137 & 1 (ref) & & 1 (ref) & \\
\hline Continuous $(25 \mathrm{~g}$ & day inta & e increme & & & 1.08 & $(0.60-1.94)$ & 1.10 & $(0.60-2.01)$ \\
\hline Chicken ${ }^{c, d}$ & & & & & & & & \\
\hline Non consumers & 0 & 0 & 56451 & 137 & 0.91 & $(0.70-1.17)$ & 0.96 & $(0.74-1.25)$ \\
\hline $\mathrm{C} 1$ & 5.3 & 5.3 & 39581 & 97 & 0.91 & (0.69-1.20) & 0.94 & $(0.72-1.24)$ \\
\hline $\mathrm{C} 2$ & 13.2 & 13.2 & 36198 & 95 & 0.97 & $(0.74-1.28)$ & 0.97 & $(0.74-1.28)$ \\
\hline $\mathrm{C} 3$ & 22.8 & 22.8 & 39645 & 108 & 1 (ref) & & 1 (ref) & \\
\hline$P_{\text {trend }}$ & & & & & 0.40 & & 0.73 & \\
\hline Continuous $(50 \mathrm{~g}$ & day inta & e increme & & & 0.96 & $(0.69-1.33)$ & 0.89 & $(0.64-1.25)$ \\
\hline Processed meat $(\mathrm{g} / \mathrm{c}$ & & & & & & & & \\
\hline Non consumers & 0 & 0 & 43461 & 98 & 0.80 & $(0.61-1.04)$ & 0.80 & $(0.59-1.09)$ \\
\hline $\mathrm{T} 1$ & 3.9 & 2.3 & 42977 & 100 & 0.79 & $(0.60-1.03)$ & 1.78 & $(0.59-1.03)$ \\
\hline $\mathrm{T} 2$ & 13.2 & 8.4 & 43249 & 117 & 0.93 & $(0.72-1.19)$ & 0.91 & $(0.70-1.18)$ \\
\hline T3 & 30.8 & 20.3 & 42188 & 122 & 1 (ref) & & 1 (ref) & \\
\hline$P_{\text {trend }}$ & & & & & 0.05 & & 0.09 & \\
\hline Continuous $(25 \mathrm{~g}$ ) & day inta & e increme & & & 1.13 & $(0.97-1.31)$ & 1.12 & $(0.95-1.33)$ \\
\hline
\end{tabular}




\begin{tabular}{|c|c|c|c|c|c|c|c|c|}
\hline \multirow[b]{2}{*}{ Food item } & \multicolumn{2}{|c|}{ Median intake } & \multirow[b]{2}{*}{ PY } & \multicolumn{5}{|c|}{ Colorectal cancer } \\
\hline & Men & Women & & Cases & $\mathrm{HR}$ & $(95 \% \mathrm{Cl})^{\mathrm{a}}$ & $\mathrm{HR}$ & $(95 \% \mathrm{Cl})^{\mathrm{b}}$ \\
\hline \multicolumn{9}{|l|}{ Fish $^{\mathrm{e}}$} \\
\hline Non consumers & 0 & 0 & 53879 & 113 & 0.83 & $(0.62-1.10)$ & 0.86 & $(0.65-1.15)$ \\
\hline C1 & 4.6 & 4.6 & 37645 & 112 & 1.17 & $(0.88-1.55)$ & 1.19 & $(0.90-1.58)$ \\
\hline $\mathrm{C} 2$ & 14.8 & 14.8 & 48056 & 128 & 1.04 & $(0.78-1.36)$ & 1.03 & $(0.78-1.36)$ \\
\hline $\mathrm{C} 3$ & 32.8 & 30.2 & 32295 & 84 & 1 (ref) & & 1 (ref) & \\
\hline$P_{\text {trend }}$ & & & & & 0.21 & & 0.39 & \\
\hline \multicolumn{4}{|c|}{ Continuous ( $25 \mathrm{~g} /$ day intake increment) } & 1.06 & & $(0.97-1.15)$ & 0.95 & $(0.82-1.09)$ \\
\hline
\end{tabular}

PY (person years at risk).

${ }^{a}$ Adjusted for age (y) and sex

${ }^{\mathrm{b}}$ Adjusted for age (y), sex, total energy intake (kcal), cigarette smoking (never, ever, current), alcohol consumption (g/day), BMI $\left(\mathrm{kg} / \mathrm{m}^{2}\right)$, non-occupational physical activity $(<30,30-60,60-90,>90 \mathrm{~min} / \mathrm{d})$, and level of education (lower vocational, second and medium vocational, university and higher vocational). If applicable, additionally adjusted for complementary meat groups holding total meat constant.

${ }^{c}$ Intake based on raw meat weight.

${ }^{d}$ Categories of intake $0,>0-<6.6, \geq 6.6-<22.8$, and $\geq 22.8 \mathrm{~g} /$ day.

${ }^{\mathrm{e}}$ Categories of intake: $0,>0-<10, \geq 10-<20$, and $\geq 20$ g/day.

\section{DISCUSSION}

Results from this prospective cohort study showed a modest, non-significantly decreased risk of colorectal cancer for vegetarians, pescetarians, and 1 day/week meat eaters compared to 6-7 day/week meat consumers.

Although the risk of colorectal and especially rectal cancer was lower in vegetarians than in non-vegetarians, this was not statistically significant. Nonetheless, our null findings are in line with a report from the Oxford Vegetarian Study [1], and a pooled analysis combining data from two prospective studies in the United Kingdom [3]. Colorectal cancer mortality also did not differ between vegetarians and non-vegetarians in a collaborative analysis of five protective cohort studies [15]. In contrast, the colorectal cancer rate was higher among vegetarians than non-vegetarians in the EPIC-Oxford study, but this cohort included a relative health conscious population of non-vegetarians [4]. The Adventist Health Study-I reported that vegetarians had a significantly lower risk of colorectal cancer than nonvegetarians [2] (average meat consumption of 3.5 servings/week), and the absence of tobacco and limited use of alcohol among this population reduces the likelihood of confounding by these factors. However, in line with other studies, we previously reported that vegetarianism is not merely characterized by a diet void of all flesh foods, but rather extends into a complete healthy diet and lifestyle [8].

We examined to what extent the associations between the diet-groups could be explained by other factors than the frequency of meat consumption. After adjusting the analyses for major risk factors of colorectal cancer such as total cigarette smoking, alcohol consumption, BMI, and physical activity, our results attenuated slightly suggesting that the lower risk observed in vegetarians and low meat consumers was only partly due to these lifestyle differences between meat consumption groups. 


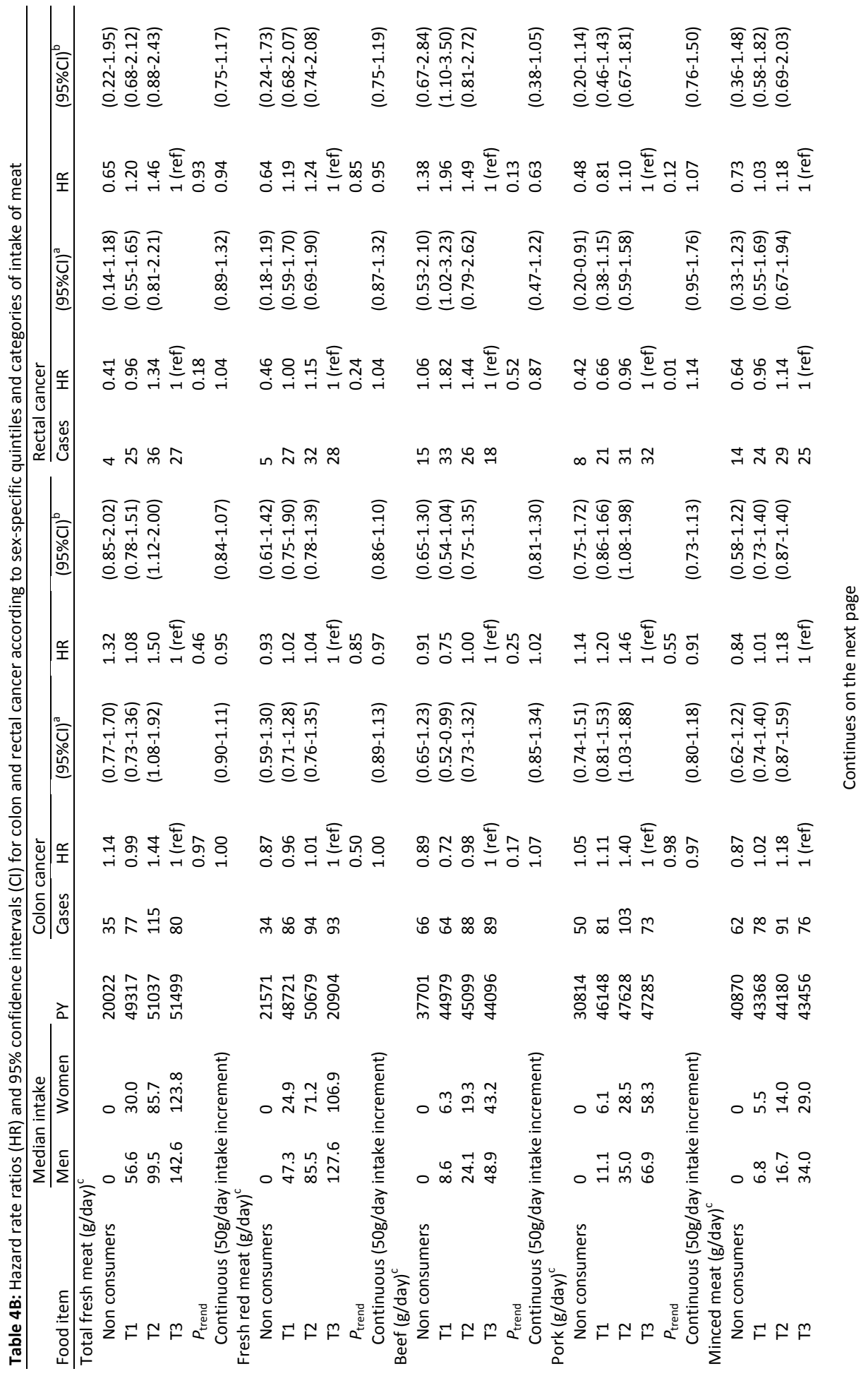




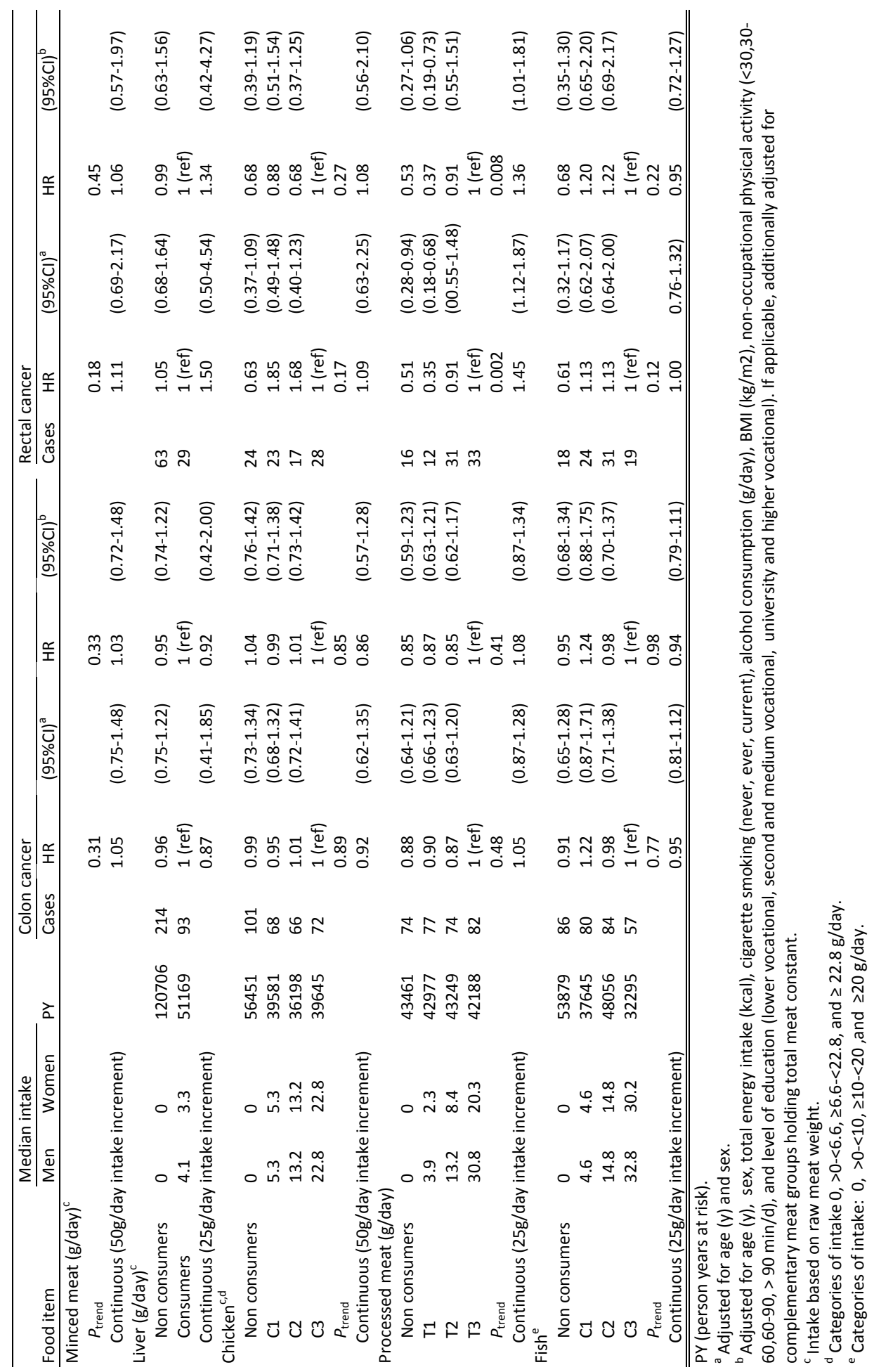


While it has been proposed that the lower disease risk in vegetarians may be explained by selective factors related to who chooses to become and remain a vegetarian [16], adjustment for e.g. level of education did not change the estimates notably either. In contrast, fiber accounted for the greatest change in HRs when comparing vegetarians and low meat consumers to 6-7 day/week meat consumers. In this respect, the WCRF expert panel concluded that there is convincing evidence that dietary fiber protects against colorectal cancer [17]. All other dietary factors that characterize a vegetarian and low meat diet had negligible individual effects, but when combined they attenuated the risk estimates, by at least 17 percent, approaching the null. This suggests that the effect of a single food group or lifestyle variable may be too small to detect, but the cumulative cancer preventive effects of multiple connected dietary and non-dietary factors may be sufficiently large to be demonstrable [18].

No study previously examined the effect of very low meat diets on colorectal cancer incidence. Our findings suggest that especially very low meat consumers may have a reduced risk of colorectal cancer compared to frequent meat consumers which was mainly observed for the colon cancer subgroup. Recent analyses from the Adventists Health StudyII suggest that, after an average of 4 years follow-up, compared to non-vegetarians the lowest risk of all gastro-intestinal cancers combined was observed among semi-vegetarians who ate red meat, poultry or fish once/month to once/week (age-adjusted HR=0.64; 95\% Cl:0.42-0.99)[5]. Whether a vegetarian diet is nutritionally adequate remains equivocal [19], and is outside the scope of this paper. It is however, interesting to observe that a strict vegetarian diet does not seem to have an additional colorectal cancer preventive effect over 1 day/week meat consumption in our population. Nonetheless, future studies with larger numbers of no and low meat consumers should replicate these findings.

Individuals adhering to a no or low meat diet often replace the meat in their diet with other (protein rich) food groups. Our observation that these individuals may have a lower colorectal cancer risk than high meat consumers is supported by our findings that substituting protein from meat with an equal percentage of energy from dairy protein significantly reduced the risk of colorectal cancer. In fact, dairy products have been hypothesized to protect against colorectal cancer risk due to their high calcium content [17, 20].

The operationalization of vegetarianism differs between studies, and the usefulness and reliability of self-reported vegetarianism in etiological studies remains unclear. For this purpose, we examined the association between vegetarianism and colorectal cancer risk using both self-definition and FFQ confirmed vegetarian status. Although both methods of classification yielded statistically non-significant protective effects, the association was considerable stronger for confirmed vegetarians than for non-vegetarians, suggesting that some attenuation occurs when merely relying on self-definition for classification purposes.

Based on our sub-site analyses, all the risk estimates appeared to be more strongly associated with rectal tumors, except for low meat consumption, which mainly decreased the risk of colon, but not rectal, cancer. Previous studies suggest that meat, subtypes of meat, and meat-related carcinogens may act differently at various locations in the colorectum $[7,21]$, possibly as a result of e.g. sub-site differences in bacterial composition 
and bacterial metabolic capacity, enzyme activity, and transit time [6, 22-24]. Although the number of especially rectal cancer cases among the vegetarians and low meat consumers in our population was low, our observation that distal colonic tumors exerted a similar pattern of association, strengthens our findings.

As a result of our sampling strategy, our population has a large contrast in meat intake which should aid the further specification of associations between subtypes of meat and colorectal cancer risk. We found no clear association between total fresh and fresh red meat intake and colorectal cancer risk. Processed meat was only associated with rectal cancer; again, comparable findings were observed for distal colon, but not for proximal colon cancer risk. Although processed meat is widely recognized risk factor for colorectal cancer $[17,25]$, this endpoint heterogeneity was not observed in a recent meta-analysis [25]. Processed meat is known to be the major source of human exposure to nitrite, and contains all the necessary precursors for $\mathrm{N}$ - nitroso compound (NOC) formation [26]; both have been specifically associated with increase rectal cancer risk only [21, 27, 28].

The NLCS attempted to enlarge the exposure contrast in the cohort by extra recruitment of vegetarian subjects [10], vegetarian dietary patterns were taken into consideration when designing the FFQ, and vegetarian status was taken into account for nutrient calculation of composite recipes. The number of vegetarians and low meat consumers in the NLCS subcohort was, however, not large enough for analyzing the effect of no and low meat dietary habits on cancer risk using the case-cohort approach that is traditionally used in NLCS-analyses. For this reason, we created the NLCS-MIC cohort - an analytical cohort over-represented with persons at the lower end of the meat consumption spectrum.

Our analyses have been performed using baseline FFQ data resulting in an inability to assess and account for changes in dietary intakes over time. However, the validity of the FFQ has been tested and shown to be representative for dietary habits over a period of at least 5 years [29]. Although we have information on time that people had adhered to their special dietary regimen at the start of follow-up (1986), stratified analyses ( $\leq 10$ years versus $>10$ years) yielded similar findings, possibly due to small numbers. The prospective design eliminates the potential for recall bias, and the nearly complete follow-up makes selection bias unlikely. Detailed information on diet and potential risk factors of colorectal cancer enabled us to control for most known risk factors, although misclassification of exposure may have occurred.

In summary, vegetarians, pescetarians, and especially 1 day/week meat eaters showed a modest, non-significantly decreased risk of colorectal cancer compared to 6-7 day/week meat consumers, mainly due to differences in dietary patterns other than meat intake.

\section{REFERENCES}

1. Sanjoaquin, M.A., et al., Nutrition, lifestyle and colorectal cancer incidence: a prospective investigation of 10998 vegetarians and non-vegetarians in the United Kingdom. Br J Cancer, 2004. 90(1): p. 118-21.

2. Fraser, G.E., Associations between diet and cancer, ischemic heart disease, and all-cause mortality in non-Hispanic white California Seventh-day Adventists. Am J Clin Nutr, 1999. 70(3 Suppl): p. 532S-538S.

3. Key, T.J., et al., Cancer incidence in British vegetarians. Br J Cancer, 2009. 101(1): p. 192-7. 
4. Key, T.J., et al., Cancer incidence in vegetarians: results from the European Prospective Investigation into Cancer and Nutrition (EPIC-Oxford). Am J Clin Nutr, 2009. 89(5): p. 1620S-1626S.

5. Tantamango-Bartley, Y., et al., Vegetarian Diets and the Incidence of Cancer in a Low-Risk Population. Cancer epidemiology, biomarkers \& prevention : a publication of the American Association for Cancer Research, cosponsored by the American Society of Preventive Oncology, 2012.

6. lacopetta, B., Are there two sides to colorectal cancer? International journal of cancer. Journal international du cancer, 2002. 101(5): p. 403-8.

7. Larsson, S.C., et al., Red meat consumption and risk of cancers of the proximal colon, distal colon and rectum: the Swedish Mammography Cohort. International journal of cancer. Journal international du cancer, 2005. 113(5): p. 829-34.

8. Gilsing A.M. et al., The Netherlands Cohort Study-Meat Investigation Cohort; a population-based cohort over-represented with vegetarians, pescetarians and low meat consumers. Nutrition journal, 2013. 12(156).

9. Willett, W.C., Convergence of philosophy and science: the third international congress on vegetarian nutrition. Am J Clin Nutr, 1999. 70(3 Suppl): p. 434S-438S.

10. van den Brandt, P.A., et al., A large-scale prospective cohort study on diet and cancer in The Netherlands. J Clin Epidemiol, 1990. 43(3): p. 285-95.

11. Van den Brandt, P.A., et al., Development of a record linkage protocol for use in the Dutch Cancer Registry for Epidemiological Research. International journal of epidemiology, 1990. 19(3): p. 553-8.

12. Schouten, L.J., J.J. Jager, and P.A. van den Brandt, Quality of cancer registry data: a comparison of data provided by clinicians with those of registration personnel. British journal of cancer, 1993. 68(5): p. 974-7.

13. Goldbohm, R.A., et al., Validation of a dietary questionnaire used in a large-scale prospective cohort study on diet and cancer. Eur J Clin Nutr, 1994. 48(4): p. 253-65.

14. Willett W., Nutritional Epidemiology. 1998, New York: Oxford University Press.

15. Key, T.J., et al., Mortality in vegetarians and nonvegetarians: detailed findings from a collaborative analysis of 5 prospective studies. Am J Clin Nutr, 1999. 70(3 Suppl): p. 516S-524S.

16. Phillips, R.L., Role of life-style and dietary habits in risk of cancer among seventh-day adventists. Cancer research, 1975. 35(11 Pt. 2): p. 3513-22.

17. World Cancer Research Fund/American Institute for Cancer Research, Food, Nutrition, Physical Activity, and the Prevention of Cancer: a Global Perspective. 2007, AICR: Washington DC.

18. Hu, F.B., Dietary pattern analysis: a new direction in nutritional epidemiology. Current opinion in lipidology, 2002. 13(1): p. 3-9.

19. Craig, W.J., Nutrition concerns and health effects of vegetarian diets. Nutrition in clinical practice : official publication of the American Society for Parenteral and Enteral Nutrition, 2010. 25(6): p. 61320.

20. Kampman, E., et al., Fermented dairy products, calcium, and colorectal cancer in The Netherlands Cohort Study. Cancer research, 1994. 54(12): p. 3186-90.

21. Cross, A.J., et al., A large prospective study of meat consumption and colorectal cancer risk: an investigation of potential mechanisms underlying this association. Cancer research, 2010. 70(6): $p$. 2406-14.

22. Mercurio, M.G., et al., Expression of cytochrome P450 mRNAs in the colon and the rectum in normal human subjects. Biochemical and biophysical research communications, 1995. 210(2): p. 350-5.

23. Macfarlane, G.T. and S. Macfarlane, Human colonic microbiota: ecology, physiology and metabolic potential of intestinal bacteria. Scandinavian journal of gastroenterology. Supplement, 1997. 222: p. 39.

24. Macfarlane, G.T., G.R. Gibson, and J.H. Cummings, Comparison of fermentation reactions in different regions of the human colon. The Journal of applied bacteriology, 1992. 72(1): p. 57-64.

25. Chan, D.S., et al., Red and processed meat and colorectal cancer incidence: meta-analysis of prospective studies. PloS one, 2011. 6(6): p. e20456.

26. Hecht, S.S., Approaches to cancer prevention based on an understanding of N-nitrosamine carcinogenesis. Proceedings of the Society for Experimental Biology and Medicine. Society for Experimental Biology and Medicine, 1997. 216(2): p. 181-91.

27. Loh, Y.H., et al., N-Nitroso compounds and cancer incidence: the European Prospective Investigation into Cancer and Nutrition (EPIC)-Norfolk Study. The American journal of clinical nutrition, 2011. 93(5): p. 1053-61. 
28. Le Marchand, L., et al., Red meat intake, CYP2E1 genetic polymorphisms, and colorectal cancer risk. Cancer epidemiology, biomarkers \& prevention : a publication of the American Association for Cancer Research, cosponsored by the American Society of Preventive Oncology, 2002. 11(10 Pt 1): p. 1019-24.

29. Goldbohm, R.A., et al., Reproducibility of a food frequency questionnaire and stability of dietary habits determined from five annually repeated measurements. Eur J Clin Nutr, 1995. 49(6): p. 420-9. 


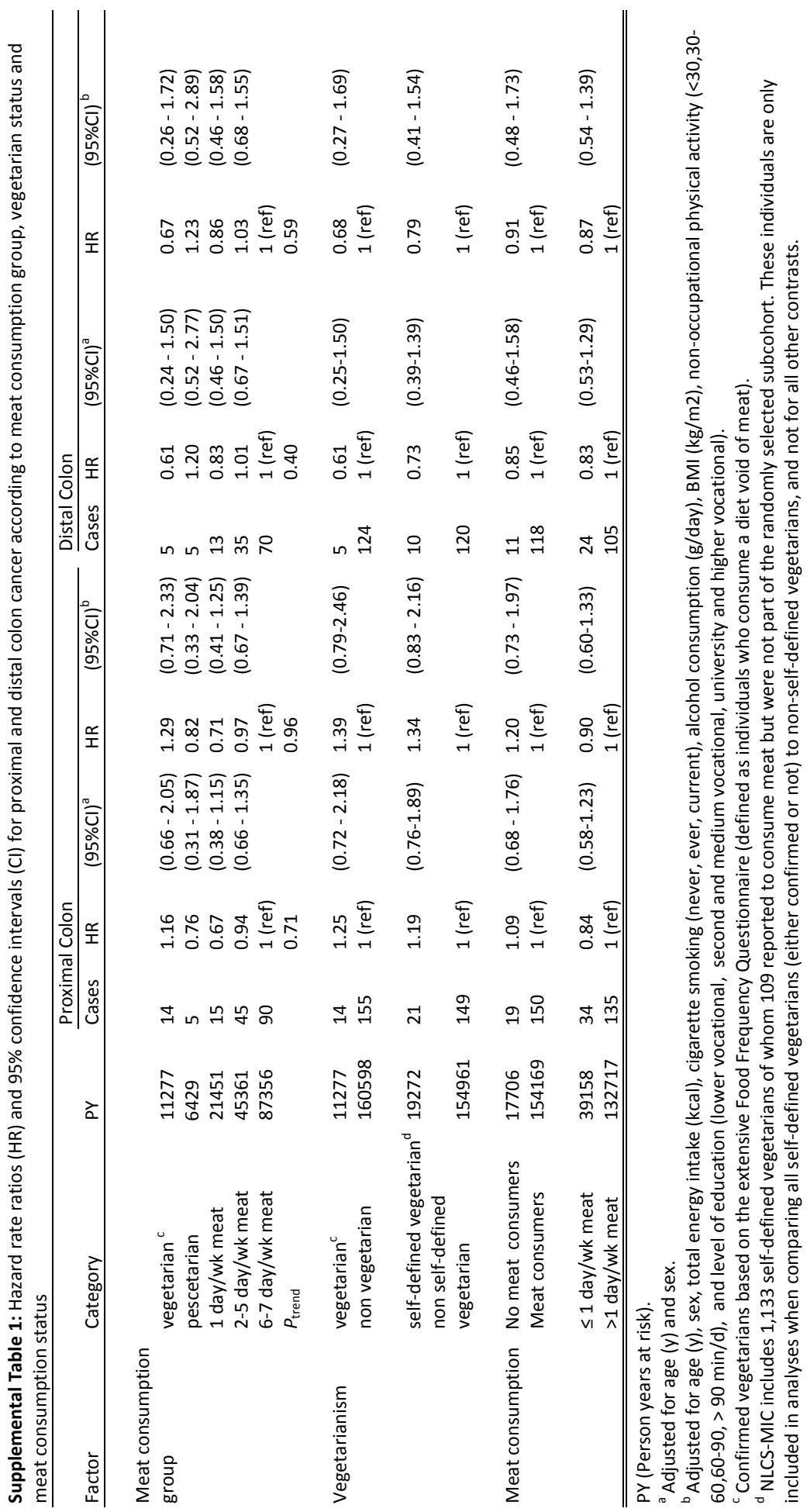




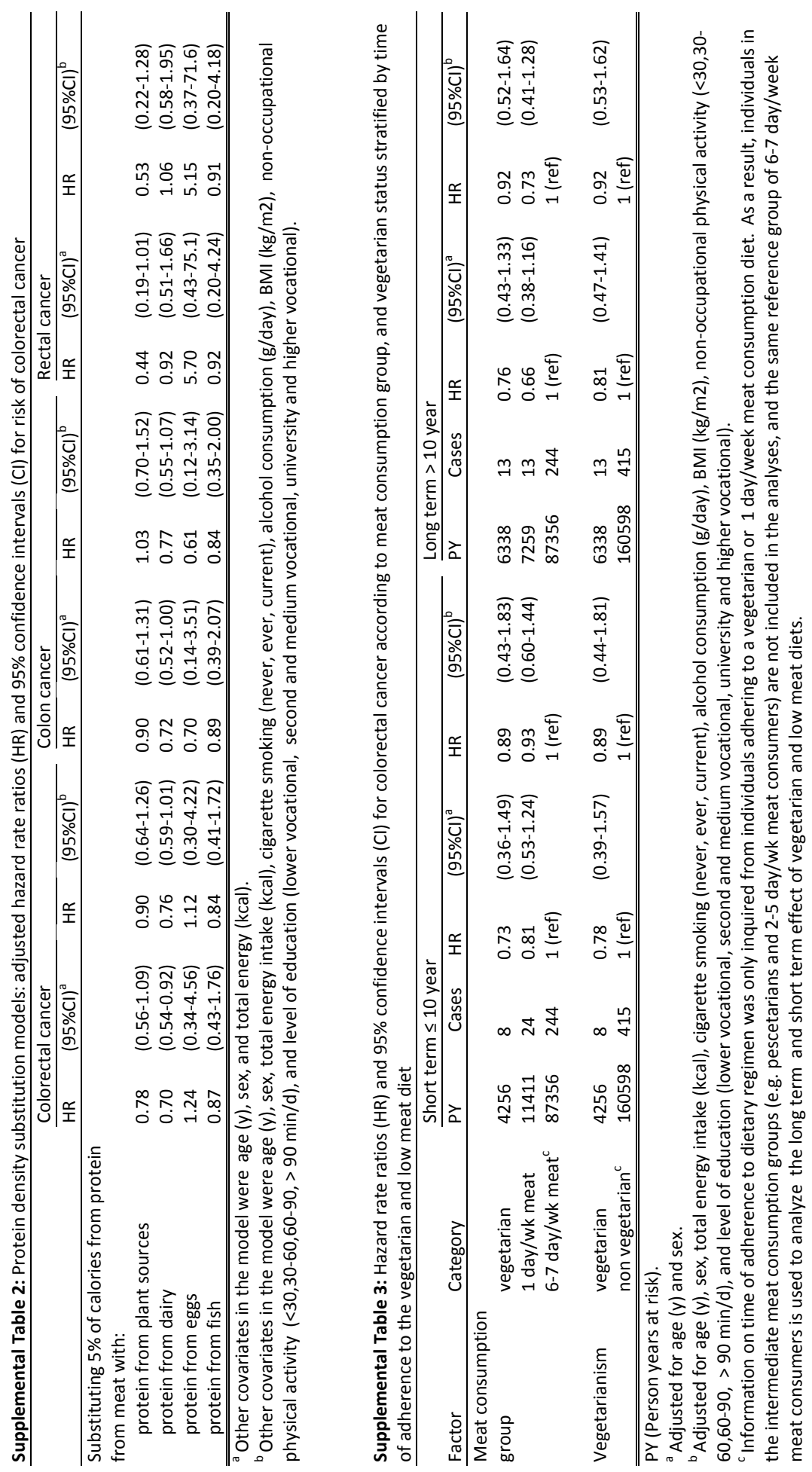





\section{CHAPTER 6}

\section{Vegetarianism, low meat consumption and the risk of lung, female breast, and prostate cancer in a population based cohort study}

Anne M.J. Gilsing Matty P. Weijenberg

R. Alexandra Goldbohm

Pieter C. Dagnelie

Piet A. van den Brandt and Leo J. Schouten

Adapted version submitted for publication 


\section{ABSTRACT}

Background: The few prospective studies that examined lung, female breast and prostate cancer risk in vegetarians have yielded mixed results, while none have studied the effects of low meat diets. In addition, little is known about the explanatory role of (non-)dietary factors associated with these diets.

Methods: The Netherlands Cohort Study - Meat Investigation Cohort (NLCS-MIC) is an analytical cohort of 11082 individuals including 1040 self-reported vegetarians. At baseline (1986), subjects completed a questionnaire on dietary habits and other risk factors for cancer and were classified into vegetarians $(n=691)$, pescetarians $(n=389), 1$ day/week( $n=1388), 2-5$ day/week- $(n=2965)$, and 6-7 day/week meat consumers $(n=5649)$. After 20.3 years of follow-up, 279 lung, 312 female breast, and 399 prostate cancer cases (including 136 advanced) were available for analyses.

Results: In age- and sex adjusted analyses, a statistically significant reduced risk of lung cancer for vegetarians and pescetarians was found when compared with 6-7 days/week meat consumers (HR: $0.44,95 \% \mathrm{Cl}: 0.21-0.94$, and HR: $0.28,95 \% \mathrm{Cl}: 0.09-0.88$ respectively). Further adjustment for confounding (especially smoking) attenuated these associations such that they were no longer significant (HR: 0.85 and 0.54, respectively). No associations were observed for female breast- and overall prostate cancer. After adjustment for confounders individuals consuming meat 1 day/week were at a 75\% increased risk of advanced prostate cancer compared to 6-7 days/week meat consumers ( $95 \% \mathrm{Cl} 1.03-2.97)$.

Conclusion: Vegetarians, pescetarians and 1 day/week meat consumers did not have a reduced risk of lung, female breast and overall prostate cancer compared to individuals consuming meat on a daily basis after taking confounders into account. Our observation that $\leq 1$ day/week meat consumption was associated with increased risk of advanced prostate cancer warrants investigation in other studies. 


\section{INTRODUCTION}

Although vegetarian diets are primarily defined by the absence of meat and fish, they are also shown to be associated with high intakes of fruits and vegetables, and a favorable distribution of non-dietary factors [1, 2]. Consequently, vegetarian diets may reduce the risk of different types of cancers through multiple mechanisms depending on the etiology and preventability of the tumor $[3,4]$.

We previously reported a non-significantly reduced risk of vegetarian and low meat diets on colorectal, and especially rectal, cancer [5] and set out to study its effect on three other major cancers. Although meat consumption has been hypothesized to be implicated in the etiology of lung, female breast and prostate cancer, data are not consistent across studies and meat subtypes [6-8]. However, based on the existing body of literature, vegetarians may be at a lower risk of developing lung cancer (due to lower smoking rates), and to female post-menopausal breast cancer (due to lower alcohol consumption, lower BMI and higher physical activity levels). While the role of diet in the etiology of prostate cancer remains poorly understood [4, 9], incidence levels of, especially localized, prostate cancers may be related to prostate specific antigen (PSA) screening utilization which was shown to differ between vegetarians and non-vegetarians [10]. Nonetheless, the few prospective cohort studies that intentionally included a large proportion of vegetarians, reported mixed and inconsistent results regarding lung, female breast and prostate cancer risk [11-15].

Although the above findings may partly reflect international differences in vegetarian diets, some of these inconsistencies may also be due to differences in population sampling strategies. Furthermore, it has been argued that advanced stage prostate cancer is different from non-advanced prostate cancer [16], but effects of vegetarian diets for these subtypes of prostate cancer have not been previously reported. In addition, studies with a large number of vegetarians and low meat eaters offer the opportunity to examine whether complete abstinence of meat might have an additional cancer preventive effect over low meat consumption, or vice versa.

Within the "Netherlands Cohort Study-Meat Investigation Cohort" (NLCS-MIC), we investigated the association of vegetarianism and (low) meat consumption with the risk of lung, female breast, and prostate cancers, including prostate cancer subgroups by disease stage. We investigated the effect of time of adherence to the dietary regimen, the reliability of self-reported vegetarianism, and the contribution of individual dietary and lifestyle factors to the observed risk estimates.

\section{METHODS}

\section{Study population and cancer follow-up}

The NLCS 'Meat Investigation Cohort' (NLCS-MIC) is an analytical cohort embedded in the ongoing prospective Netherlands Cohort Study (NLCS). The total NLCS study was initiated in September 1986 and includes 120852 men and women aged 55-69 years at baseline. All of the women were presumed to be postmenopausal. At the start of the study, participants completed a self-administered questionnaire on dietary habits, lifestyle characteristics, medical history, and other potential risk factors for cancer [17]. Because the NLCS 
traditionally uses the case-cohort design for efficiency; questionnaire data were only available for a randomly selected subcohort and all cancer cases. Therefore, NLCS-MIC is specifically extracted from the total NLCS to study the health effects of vegetarian and low meat diets and consists of the random subcohort of 10000 subjects and all self-reported vegetarians $(n=1040)$ and 1 day/week meat consumers from the total NLCS cohort. The latter were selected based on two questions on the first page of the questionnaire that was processed for all 120852 cohort members: "Do you have any special eating habits?", and "How many days on average per week do you eat meat?" The 150 item food frequency questionnaire (FFQ) was used to categorize NLCS-MIC ( $n=11082)$ into five meat consumption categories: confirmed vegetarians $(n=691)$ and pescetarians $(n=389), 1$ day/week- $(n=1388), 2-5$ days/week- $(n=2965)$, and 6-7 days/week meat consumers $(n=5649)$. Full details of the study design have been described elsewhere [1]. The NLCS has been approved by the institutional review boards of the TNO Quality of Life Research Institute (Zeist, the Netherlands) and Maastricht University (Maastricht, the Netherlands).

\section{Questionnaire}

The FFQ estimated the average frequency and amount of foods and beverages consumed over 12 months preceding baseline. The questionnaire also assessed the time since the start of any special eating habits and weekly frequency of meat consumption (for 0-1 day/week meat consumers), in years until baseline (1986). In addition, the FFQ contained 14 items on the consumption of meat with the hot meal (mainly fresh meat, including chicken), 5 items on the consumption of meat products used as sandwich fillings, and 3 items on fish consumption (with the hot meal, for lunch, as a snack in between meals).

\section{Statistical analyses}

We estimated the association between meat consumption group (confirmed vegetarian, pescetarian, 1 day/week-, 2-5 days/week-, and 6-7 days/week meat consumers (reference group)) and the risk of lung, female breast, and prostate cancer. In addition, the association of cancer incidence with self-reported vegetarian status (self-reported versus complementary group of non-self-reported-vegetarians) and confirmed vegetarian status (confirmed vegetarian versus complementary group of non-vegetarians) was examined. In case a statistically significant association was observed, we assessed to what extent this association could be explained by other dietary and lifestyle variables associated with a vegetarian or low meat (i.e. meat for 1 day/week) diet. Therefore, we calculated the percentage change in risk estimate, firstly adjusting for age and sex, then further adjusting for energy and each food group, or lifestyle factor in turn. Furthermore, the association with meat consumption group and confirmed vegetarian status was stratified by duration of adherence to the specific diet ( $\leq 10$ years, $>10$ years).

The following food groups and foods were also selected for analyses (in g/day): fresh meat (beef, pork, minced meat, chicken, liver), processed meat, fish, fresh red meat (fresh meat without chicken) and beef, pork, minced meat, chicken, and liver as separate types. For the individual meat types, subjects were classified into non-consumers, and tertiles of consumers (highest tertile as reference group), and as continuous variables (per 25 or 50 gram increase). For some meat types, categories were used instead of tertiles (liver: a nonuser and a user group (>0g/day); chicken: $0,0-<6.6, \geq 6.6-<22.8$ and $\geq 22.8 \mathrm{~g} /$ day; fish $0,0-$ $<10, \geq 10-<20$ and $\geq 20 \mathrm{~g} /$ day). 
For all the above described contrasts, age and sex adjusted and multi-variable adjusted hazard rate ratios (HRs) and their corresponding 95\% confidence intervals $(95 \% \mathrm{Cl})$ were estimated using Cox proportional hazards models. The proportional hazards assumption was tested using the scaled Schoenfeld residuals. If there was an indication for violation of the assumption for a variable, we further investigated this by adding a time-varying covariate for that variable to the model. Sensitivity analyses excluding the first 2 years of follow-up were performed. The covariates included in the multivariable analyses were either a priori selected risk factors of lung, female breast and prostate cancer, or variables that changed the risk estimates for meat consumption group, vegetarian status or total fresh meat intake by $10 \%$ or more. For all investigated cancers, this resulted in a final model including age (years), total energy intake (kcal/day), cigarette smoking (never/ever/current), alcohol consumption (g/day), BMI $\left(\mathrm{kg} / \mathrm{m}^{2}\right)$, non-occupational physical activity $(\leq 30 />30-\leq 60 />60-\leq 90 />90$ minutes/day), and level of education (lower vocational, secondary-/medium-vocational, university and higher vocational). Lung cancer analyses were additionally adjusted for sex, frequency of smoking (n/day), and duration of smoking (years). Associations for female breast cancer were additionally adjusted for height $(\mathrm{cm})$, age at menarche (years), age at first child (years), number of children, age at menopause $(<50$ years $/ \geq 50$ years/unknown), oral contraceptive use (never/ever), hormone replacement therapy (yes/no/don't know), and first-grade family history of breast cancer (yes/no). Prostate cancer analyses were also adjusted for first-grade family history of prostate cancer. The independent contribution of the individual meat categories was examined by constructing addition models that summed to total meat.

To enable comparison, the age (and sex)-adjusted analyses were restricted to subjects included in multivariable-adjusted models. After 20.3 years of follow-up and exclusion of prevalent cancer cases at baseline, 279 lung cancer cases (ICD-O C34), 312 female breast cancer cases (C50), and 399 prostate cancer cases (C61) (including 136 advanced (TNM stage III/IV: T3+, $\mathrm{N}+$, or $\mathrm{M} 1$ at diagnosis)) remained eligible for analyses. Linear trends were evaluated with the Wald test by entering the categorical exposure variables as a continuous term in the Cox regression model.

All tests were two-tailed and differences were regarded as statistically significant at $P<0.05$. All analyses were performed using STATA Statistical Software (Intercooled STATA, version 12; Stata-Corp LP, College Station, TX).

\section{RESULTS}

The distribution of demographic and (non-) dietary characteristics according to meat consumption group has already been extensively described [1]. Baseline characteristics for cases and non-cases for the cancers under study are described in Table 1. 
Table 1: Baseline characteristics (means or percent) and dietary intakes of exposures of interest of lung, female breast, and lung cancer cases and non-cases in the NLCS-MIC, 1986-2006.

\begin{tabular}{|c|c|c|c|c|c|c|c|}
\hline \multirow[b]{2}{*}{ Characteristics } & \multicolumn{2}{|l|}{ Lung Cancer } & \multicolumn{2}{|c|}{ Female Breast Cancer } & \multicolumn{3}{|c|}{ Prostate Cancer } \\
\hline & Non cases & Cases & Non cases & Cases & Non cases & $\begin{array}{l}\text { All Prostate } \\
\text { cancer cases }\end{array}$ & $\begin{array}{l}\text { Advanced } \\
\text { prostate } \\
\text { cancer cases }\end{array}$ \\
\hline $\mathrm{N}$ & 9494 & 279 & 4906 & 312 & 4465 & 399 & 136 \\
\hline \multicolumn{8}{|l|}{$\begin{array}{l}\text { Meat consumption } \\
\text { group }(\%)\end{array}$} \\
\hline Vegetarian & $6 \%$ & $3 \% *$ & $8 \%$ & $6 \%$ & $4 \%$ & $5 \%$ & $5 \%$ \\
\hline Pescetarian & $4 \%$ & $1 \%$ & $4 \%$ & $5 \%$ & $3 \%$ & $4 \%$ & $5 \%$ \\
\hline $1 \mathrm{day} / \mathrm{wk}$ meat & $13 \%$ & $10 \%$ & $15 \%$ & $17 \%$ & $9 \%$ & $10 \%$ & $14 \%$ \\
\hline 2-5 days/wk meat & $27 \%$ & $26 \%$ & $27 \%$ & $8 \%$ & $26 \%$ & $26 \%$ & $23 \%$ \\
\hline 6-7 days/wk meat & $51 \%$ & $61 \%$ & $45 \%$ & $45 \%$ & $59 \%$ & $55 \%$ & $53 \%$ \\
\hline Sex (\% men) & $45 \%$ & $85 \% *$ & $0 \%$ & $0 \%$ & $100 \%$ & $100 \%$ & $100 \%$ \\
\hline Age $(y)$ & $61.3 \pm 4.2$ & $62.3 \pm 4.2$ & $61.4 \pm 4.2$ & $61.2 \pm 4.3$ & $61.2 \pm 4.2$ & $62.1 \pm 4.1^{*}$ & $62.2 \pm 4.0^{*}$ \\
\hline Current smokers (\%) & $24 \%$ & $66 \% *$ & $18 \%$ & $17 \%$ & $34 \%$ & $28 \%$ & $23 \% *$ \\
\hline BMI (mean) & $24.7 \pm 3.2$ & $24.6 \pm 2.8$ & $24.6 \pm 3.6$ & $25.1 \pm 3.5^{*}$ & $24.8 \pm 2.7$ & $24.7 \pm 2.2$ & $24.9 \pm 2.2$ \\
\hline \multicolumn{8}{|c|}{ Physical activity (non-occupational) (\%) } \\
\hline$<30 \mathrm{~min} /$ day & $21 \%$ & $23 \%$ & $23 \%$ & $25 \%$ & $18 \%$ & $18 \%$ & $13 \%$ \\
\hline $30-60 \mathrm{~min} / \mathrm{day}$ & $30 \%$ & $32 \%$ & $31 \%$ & $32 \%$ & $30 \%$ & $25 \%$ & $28 \%$ \\
\hline $60-90 \mathrm{~min} / \mathrm{day}$ & $23 \%$ & $16 \%$ & $24 \%$ & $22 \%$ & $20 \%$ & $26 \%$ & $26 \%$ \\
\hline$>90 \mathrm{~min} /$ day & $26 \%$ & $28 \%$ & $22 \%$ & $20 \%$ & $31 \%$ & $31 \%$ & $34 \%$ \\
\hline \multicolumn{8}{|c|}{ Level of education (\%) } \\
\hline Low & $46 \%$ & $53 \% *$ & $50 \%$ & $42 \% *$ & $42 \%$ & $39 \% *$ & $38 \%$ \\
\hline Medium & $37 \%$ & $35 \%$ & $38 \%$ & $47 \%$ & $36 \%$ & $31 \%$ & $34 \%$ \\
\hline High & $17 \%$ & $12 \%$ & $12 \%$ & $11 \%$ & $22 \%$ & $29 \%$ & $29 \%$ \\
\hline $\begin{array}{l}\text { Supplement use } \\
\text { (\% users) }\end{array}$ & $35 \%$ & $24 \% *$ & $42 \%$ & $43 \%$ & $26 \%$ & $26 \%$ & $21 \%$ \\
\hline Energy (kcal) & $1880 \pm 518$ & $2081 \pm 492^{*}$ & $1668 \pm 412$ & $1692 \pm 406$ & $2134 \pm 512$ & $2179 \pm 546$ & $2195 \pm 474$ \\
\hline Fiber (g) & $28.2 \pm 7.6$ & $27.3 \pm 7.3^{*}$ & $26.6 \pm 6.6$ & $26.8 \pm 7.3$ & $30.0 \pm 8.1$ & $29.6 \pm 7.9$ & $29.6 \pm 8.0$ \\
\hline Alcohol (g) & $9.1 \pm 13.7$ & $15.1 \pm 16.4^{*}$ & $5.3 \pm 9.3$ & $6.8 \pm 12.0^{*}$ & $13.9 \pm 16.3$ & $14.7 \pm 17.4$ & $14.6 \pm 15.1$ \\
\hline Total fresh meat $(\mathrm{g})^{\mathrm{b}}$ & $80.4 \pm 52.4$ & $96.3 \pm 55.4$ & $71.4 \pm 50.6$ & $72.4 \pm 50.8$ & $92.1 \pm 52.7$ & $87.2 \pm 52.2$ & $81.2 \pm 53.6^{*}$ \\
\hline Fresh red meat $(\mathrm{g})^{\mathrm{c}}$ & $68.9 \pm 48.8$ & $86.2 \pm 52.9^{*}$ & $61.3 \pm 46.6$ & $62.2 \pm 46.3$ & $81.3 \pm 49.6$ & $76.8 \pm 48.9$ & $72.3 \pm 49.5^{*}$ \\
\hline Beef $(g)$ & $21.3 \pm 23.5$ & $26.5 \pm 29.1^{*}$ & $18.8 \pm 22.3$ & $19.4 \pm 20.7$ & $24.7 \pm 25.1$ & $23.3 \pm 24.0$ & $23.3 \pm 25.4$ \\
\hline Pork (g) & $30.1 \pm 29.6$ & $37.5 \pm 31.7^{*}$ & $26.6 \pm 28.1$ & $26.2 \pm 27.5$ & $35.1 \pm 31.0$ & $33.6 \pm 29.6$ & $31.7 \pm 28.4$ \\
\hline Minced meat (g) & $14.8 \pm 16.1$ & $17.7 \pm 20.6^{*}$ & $12.8 \pm 14.6$ & $13.2 \pm 14.6$ & $17.2 \pm 17.8$ & $16.1 \pm 17.8$ & $14.7 \pm 14.5$ \\
\hline Liver (g) & $1.6 \pm 4.0$ & $2.2 \pm 4.9$ & $1.3 \pm 3.5$ & $1.6 \pm 4.1$ & $1.9 \pm 4.4$ & $1.7 \pm 4.6$ & $0.95 \pm 2.32 *$ \\
\hline Chicken (g) & $11.4 \pm 14.9$ & $11.1 \pm 14.1$ & $10.8 \pm 14.7$ & $10.7 \pm 17.5$ & $11.9 \pm 14.7$ & $11.4 \pm 13.4$ & $9.4 \pm 12.9^{*}$ \\
\hline Processed meat (g) & $10.8 \pm 14.0$ & $15.0 \pm 18.8^{*}$ & $8.2 \pm 11.2$ & $7.2 \pm 9.1$ & $14.5 \pm 16.8$ & $12.5 \pm 15.3$ & $11.0 \pm 13.3^{*}$ \\
\hline Fish (g) & $13.0 \pm 17.9$ & $14.6 \pm 22.1$ & $11.8 \pm 16.4$ & $11.5 \pm 16.2$ & $14.4 \pm 19.4$ & $15.1 \pm 18.7$ & $14.1 \pm 19.4$ \\
\hline Vegetables (g) & $200 \pm 89$ & $190 \pm 85$ & $201 \pm 88$ & $211 \pm 112$ & $197 \pm 88$ & $200 \pm 88$ & $200 \pm 79$ \\
\hline Fruits (g) & $186 \pm 128$ & $138 \pm 113^{*}$ & $203 \pm 129$ & $213 \pm 129$ & $162 \pm 124$ & $164 \pm 118$ & $157 \pm 96$ \\
\hline Pulses (g) & $10.1 \pm 16.8$ & $9.9 \pm 14.9$ & $8.8 \pm 15.1$ & $9.4 \pm 14.1$ & $11.4 \pm 18.7$ & $11.4 \pm 15.9$ & $12.1 \pm 15.7$ \\
\hline Soya products (g) & $3.1 \pm 18.0$ & $0.99 \pm 6.1^{*}$ & $3.2 \pm 21.0$ & $3.6 \pm 17.7$ & $2.8 \pm 13.5$ & $2.7 \pm 11.8$ & $4.0 \pm 14.8$ \\
\hline Milk (g) & $313 \pm 207$ & $309 \pm 219$ & $313 \pm 200$ & $307 \pm 193$ & $311 \pm 215$ & $311 \pm 222$ & $317 \pm 194$ \\
\hline Cheese (g) & $26.0 \pm 22.6$ & $23.0 \pm 21.1^{*}$ & $25.5 \pm 21.0$ & $26.8 \pm 21.3$ & $26.1 \pm 24.1$ & $27.4 \pm 24.4$ & $26.4 \pm 21.7$ \\
\hline Eggs (g) & $15.5 \pm 11.6$ & $16.9 \pm 12.6$ & $14.5 \pm 10.7$ & $15.2 \pm 11.2$ & $16.8 \pm 12.5$ & $16.9 \pm 13.0$ & $16.0 \pm 14.8$ \\
\hline $\begin{array}{l}\text { All of the women were } \\
\text { a mean } \pm \text { SD, all such va } \\
\text { b Intake based on raw n } \\
\text { c Includes beef, pork, m } \\
\text { * Statistically significan } \\
\text { variables) }\end{array}$ & $\begin{array}{l}\text { presumed to } \\
\text { alues } \\
\text { meat weight } \\
\text { inced meat, } \\
\text { it different frc }\end{array}$ & be postmenop & oausal & & & & \\
\hline
\end{tabular}


The imbalanced sex distribution between lung cancer cases and non-cases, likely resulted in between-group differences in baseline variables (e.g. lung cancer cases were more often current smokers, had higher intakes of total kcal, alcohol, and meat, but consumed lower amounts of fiber, fruits, soy products and cheese compared to non-cases). This is also reflected in a smaller percentage of vegetarians and pescetarians among lung cancer cases compared to non-cases. No large differences between female breast cancer cases and noncases were observed. Advanced stage prostate cancer cases were older, and consumed smaller amounts of meat compared to non-cases.

After adjustment for age and sex, a statistically significant reduced risk of lung cancer for vegetarians and pescetarians was found when compared to 6-7 days/week meat consumers (HR:0.44, 95\% Cl:0.21-0.94, and HR:0.28, 95\%Cl:0.09-0.88 respectively) (Table 2A). Further adjustment for confounding attenuated these associations such that they were no longer significant (HR:0.84, 95\% $\mathrm{Cl}: 0.39-1.84$, and $\mathrm{HR}: 0.54,95 \% \mathrm{Cl}: 0.17-1.70$ respectively). A similar pattern was observed when comparing non-meat consumers to meat consumers, and vegetarians (confirmed or self-reported) to their complementary group of nonvegetarians.

There was no evidence of an interaction by sex for any of the comparisons made. However, the number of lung cancer cases among the vegetarians and pescetarians was small ( $n=7$ and $n=3$, respectively). No statistically significant associations for female breast cancer were observed in the age and multivariable adjusted models (Table 2A). Although no associations were observed between the risk of all prostate cancers and meat consumption group, a statistically significant trend across meat consumption groups was observed for advanced prostate cancer risk after adjustment for confounders ( $P=0.05)$ (Table 2B). Individuals consuming meat $\leq 1$ per week had a $67 \%$ higher risk of advanced prostate cancer compared to those consuming meat for two or more days per week $(95 \% \mathrm{Cl}: 1.10-$ 2.54). In a lag analysis excluding the first 2 years of follow-up, the findings for meat consumption group and vegetarian status did not change appreciably (data not shown).

To further unravel how confounding by diet and lifestyle factors affected the observations for lung and advanced prostate cancer we calculated the percent change in HR across the meat consumption groups, firstly adjusted for age (and sex), then further adjusted for energy and each food group, or lifestyle factor in turn (Table 3). Although statistical power was limited, smoking status and -duration, but not smoking frequency, contributed most to the observed inverse risk of lung cancer of vegetarians, pescetarians, and 1 day-week meat eaters when compared to 6-7 day-week meat eaters. Nonetheless, a model including all dietary variables combined was also able to explain most of the observed risk reduction for vegetarians, although this may largely result from residual confounding by smoking. In contrast, risk estimates for prostate cancer tended to further increase away from the null after adjustment for dietary and lifestyle factors. 


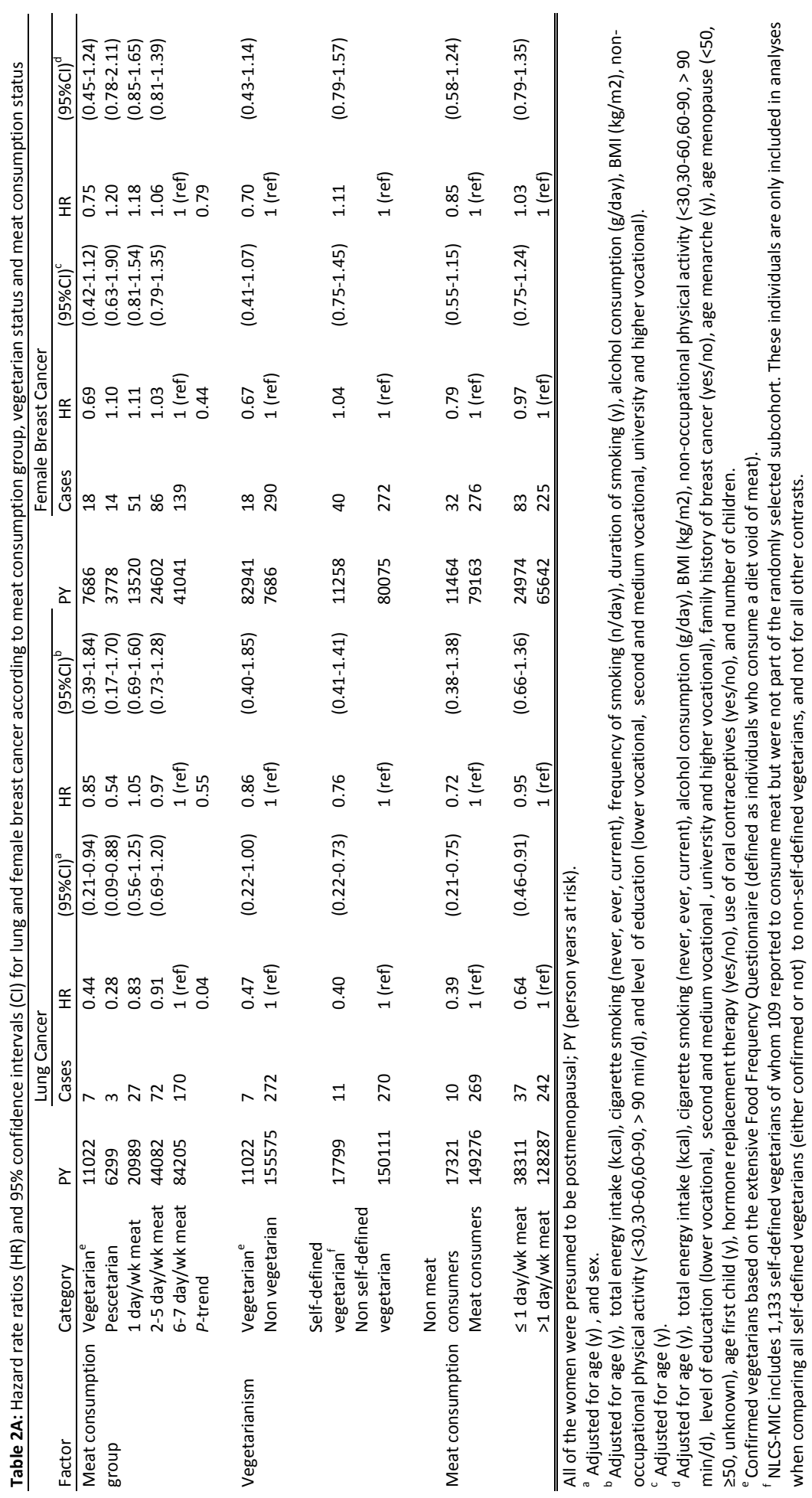




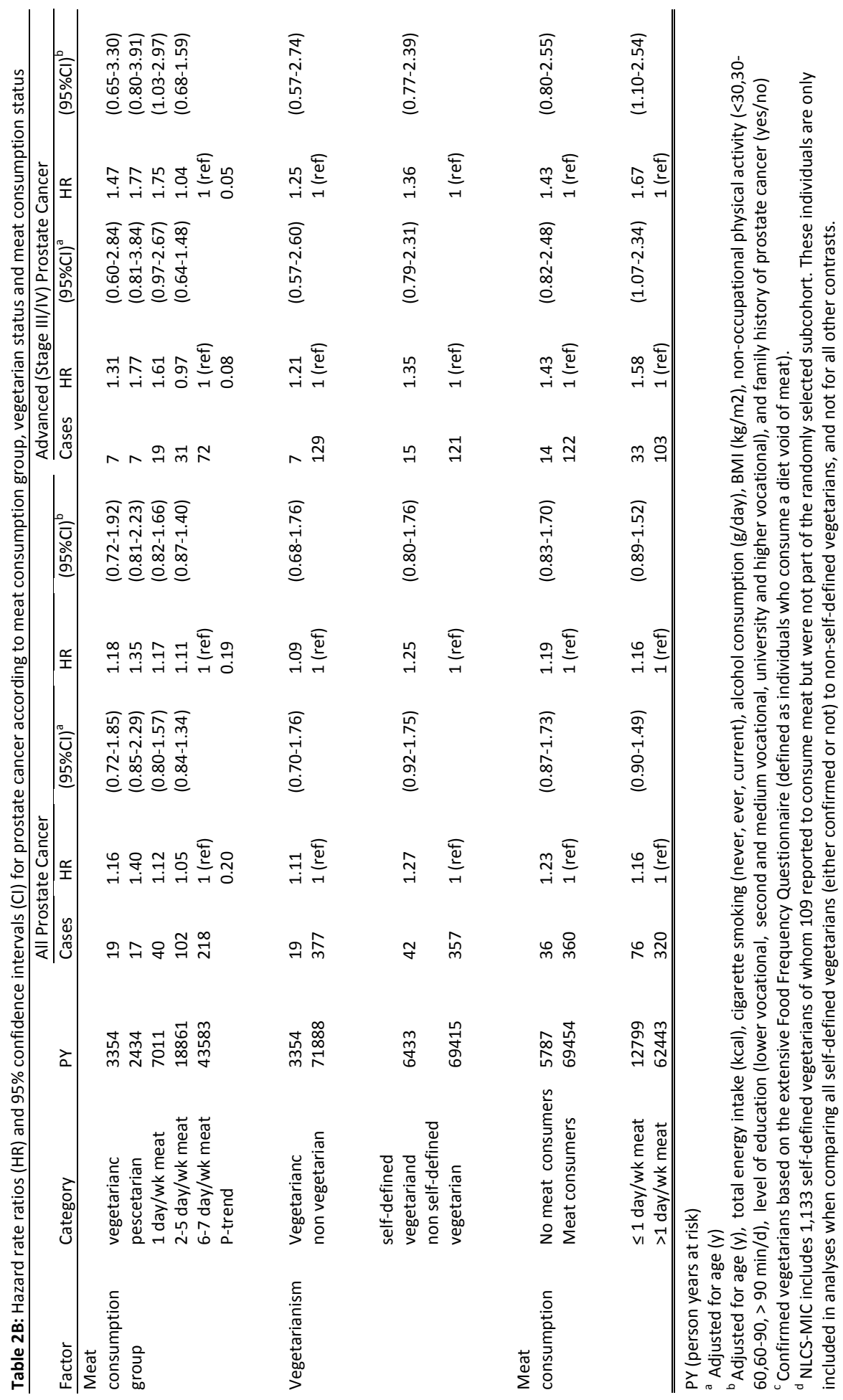




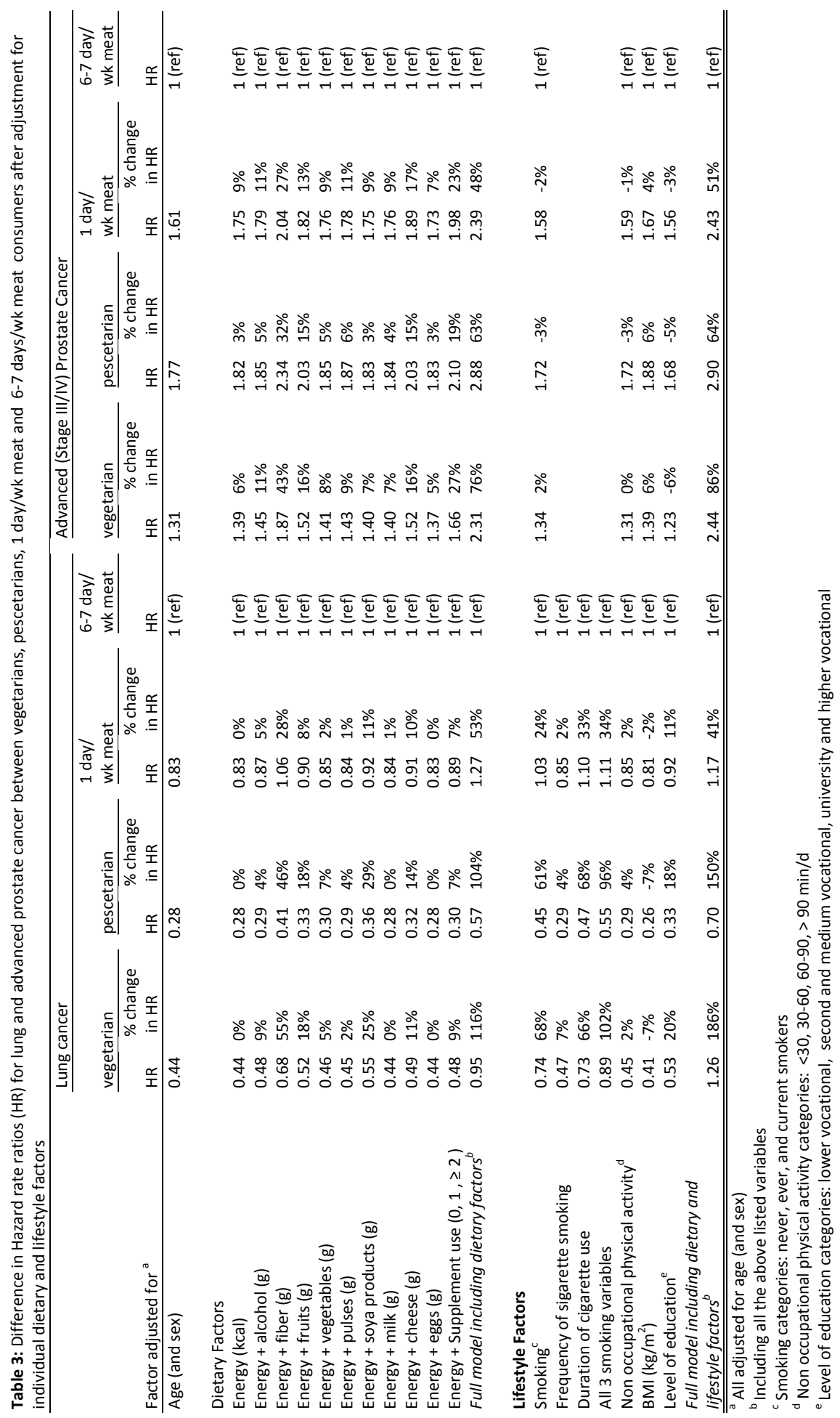


After multivariable adjustment, 1 day/week meat consumers adhering to their diet for less than 10 years were at an increased risk of female breast, lung and overall prostate cancer; although this only reach statistical significance for breast cancer. In contrast, long term adherers (> 10 years) were at a non-statistically significant reduced risk of all three cancers under study compared to 6-7 days/week meat consumers (Supplemental Table 1). The number of advanced prostate cancers was too small to present these stratified results.

Low consumption of total fresh meat, fresh red meat, beef, pork and minced meat was associated with a significantly reduced lung cancer risk in the age and sex adjusted models (Table 4A). However, these attenuated and were no longer statistically significant after adjustment for confounders. No association between breast cancer (Table 4B) and overall prostate cancer Table $\mathbf{4 C}$ ) and any of the meat items was observed. Individuals reporting not to consume chicken, and processed meat were at an increased risk of advanced prostate cancer compared to those in the highest category of consumption after adjustment for confounders (HR:1.88, 95\%Cl:1.14-3.12; HR:1.77 95\%Cl:1.00-3.14, respectively).

Table 4A: Hazard rate ratios (HR) and 95\% confidence intervals ( $\mathrm{Cl}$ ) for lung cancer according to sex-specific quintiles and categories of intake of fresh meat, types of fresh meat, and processed meat.

\begin{tabular}{|c|c|c|c|c|c|c|c|c|}
\hline \multirow[b]{2}{*}{ Food item } & \multicolumn{3}{|c|}{ Median intake } & \multicolumn{5}{|c|}{ Lung Cancer } \\
\hline & Men & Women & PY & Cases & $\mathrm{HR}$ & $(95 \% \mathrm{Cl})^{\mathrm{a}}$ & HR & $(95 \% \mathrm{Cl})^{\mathrm{b}}$ \\
\hline \multicolumn{9}{|c|}{ Total fresh meat (g/day) ${ }^{\mathrm{c}}$} \\
\hline Non consumers & 0.0 & 0.0 & 19623 & 11 & 0.37 & $(0.20-0.69)$ & 0.66 & $(0.34-1.26)$ \\
\hline $\mathrm{T} 1$ & 56.8 & 30.4 & 47777 & 89 & 0.98 & $(0.73-1.32)$ & 1.18 & $(0.87-1.61)$ \\
\hline $\mathrm{T} 2$ & 99.7 & 85.8 & 49336 & 87 & 0.96 & $(0.71-1.28)$ & 0.99 & $(0.74-1.34)$ \\
\hline T3 & 142.3 & 123.9 & 49863 & 92 & 1 (ref) & & 1 (ref) & \\
\hline \multirow{2}{*}{\multicolumn{4}{|c|}{$\begin{array}{l}P_{\text {trend }} \\
\text { Continuous }(50 \mathrm{~g} / \text { day intake increment) }\end{array}$}} & & 0.04 & & 0.90 & \\
\hline & & & & & 1.17 & $(1.05-1.31)$ & 1.03 & $(0.91-1.17)$ \\
\hline \multicolumn{9}{|c|}{ Fresh red meat $(\mathrm{g} / \mathrm{day})^{\mathrm{c}}$} \\
\hline Non consumers & 0.0 & 0.0 & 21075 & 11 & 0.35 & $(0.19-0.65)$ & 0.65 & $(0.34-1.24)$ \\
\hline T1 & 47.1 & 25.0 & 47273 & 84 & 0.94 & $(0.70-1.26)$ & 1.19 & $(0.87-1.62)$ \\
\hline $\mathrm{T} 2$ & 85.5 & 71.1 & 48965 & 93 & 1.03 & $(0.77-1.37)$ & 1.13 & $(0.84-1.52)$ \\
\hline T3 & 127.5 & 107.1 & 49285 & 91 & 1 (ref) & & 1 (ref) & \\
\hline \multicolumn{4}{|c|}{$P_{\text {trend }}$} & & 0.01 & & 0.94 & \\
\hline \multicolumn{4}{|c|}{ Continuous ( $50 \mathrm{~g} /$ day intake increment) } & & 1.21 & $(1.08-1.36)$ & 1.05 & $(0.92-1.19)$ \\
\hline \multicolumn{9}{|c|}{ Beef $(g / d a y)^{d}$} \\
\hline Non consumers & 0.0 & 0.0 & 36658 & 33 & 0.55 & $(0.37-0.88)$ & 0.66 & $(0.44-1.01)$ \\
\hline $\mathrm{T} 1$ & 8.6 & 6.3 & 43572 & 83 & 0.97 & $(0.72-1.32)$ & 1.03 & $(0.76-1.40)$ \\
\hline $\mathrm{T} 2$ & 24.2 & 19.3 & 43617 & 79 & 0.95 & $(0.70-1.29)$ & 1.02 & $(0.75-1.39)$ \\
\hline T3 & 48.8 & 43.0 & 42751 & 84 & 1 (ref) & & 1 (ref) & \\
\hline \multicolumn{4}{|c|}{$P_{\text {trend }}$} & & 0.02 & & 0.17 & \\
\hline \multicolumn{4}{|c|}{ Continuous (50g/day intake increment) } & & 1.27 & $(1.02-1.59)$ & 1.13 & $(0.90-1.42)$ \\
\hline \multicolumn{9}{|c|}{ Pork $(g / d a y)^{c}$} \\
\hline Non consumers & 0.0 & 0.0 & 30036 & 22 & 0.48 & $(0.30-0.77)$ & 0.77 & $(0.46-1.28)$ \\
\hline $\mathrm{T} 1$ & 11.1 & 6.1 & 44916 & 79 & 0.92 & $(0.68-1.26)$ & 1.14 & $(0.83-1.57)$ \\
\hline $\mathrm{T} 2$ & 35.0 & 28.6 & 46276 & 94 & 1.10 & $(0.82-1.48)$ & 1.25 & $(0.92-1.68)$ \\
\hline T3 & 67.0 & 58.1 & 45370 & 84 & 1 (ref) & & 1 (ref) & \\
\hline$P_{\text {trend }}$ & & & & & 0.007 & & 0.82 & \\
\hline \multicolumn{4}{|c|}{ Continuous (50g/day intake increment) } & & 1.25 & $(1.05-1.50)$ & 1.01 & $(0.83-1.23)$ \\
\hline
\end{tabular}




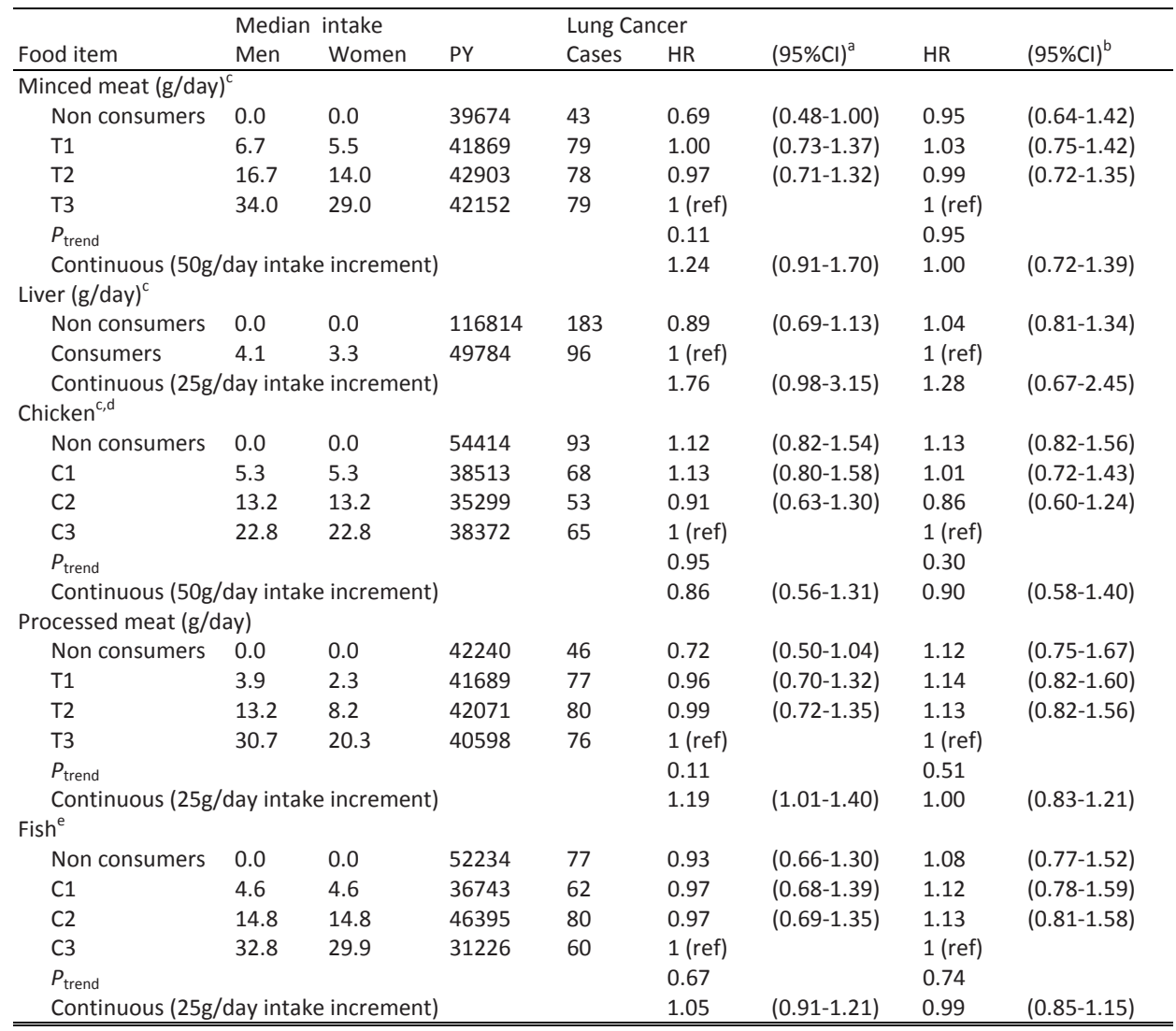

PY (person years at risk).

${ }^{\text {a }}$ Adjusted for age (y), and sex

${ }^{\mathrm{b}}$ Adjusted for age (y), total energy intake (kcal), cigarette smoking (never, ever, current), frequency of smoking ( $\mathrm{n} /$ day), duration of smoking (y), alcohol consumption (g/day), BMI $\left(\mathrm{kg} / \mathrm{m}^{2}\right)$, non-occupational physical activity $(<30,30-60,60-90,>90 \mathrm{~min} / \mathrm{d})$, and level of education (lower vocational, second and medium vocational, university and higher vocational).

${ }^{c}$ Intake based on raw meat weight.

${ }^{d}$ Categories of intake $0,>0-<6.6, \geq 6.6-<22.8$, and $\geq 22.8 \mathrm{~g} /$ day.

${ }^{\mathrm{e}}$ Categories of intake: $0,>0-<10, \geq 10-<20$, and $\geq 20 \mathrm{~g} /$ day. 
Table 4B: Hazard rate ratios (HR) and 95\% confidence intervals $(\mathrm{Cl})$ for female breast cancer according to quintiles and categories of intake of fresh meat, types of fresh meat, and processed meat.

\begin{tabular}{|c|c|c|c|c|c|c|c|}
\hline \multirow[b]{2}{*}{ Food item } & \multirow[b]{2}{*}{ Median intake } & \multirow[b]{2}{*}{ PY } & \multicolumn{5}{|c|}{ Breast Cancer } \\
\hline & & & Cases & $\mathrm{HR}$ & $(95 \% \mathrm{Cl})^{\mathrm{a}}$ & $\mathrm{HR}$ & $(95 \% \mathrm{Cl})^{b}$ \\
\hline \multicolumn{8}{|c|}{ Total fresh meat (g/day) ${ }^{\mathrm{c}}$} \\
\hline Non consumers & 0.0 & 12902 & 36 & 0.82 & $(0.55-1.20)$ & 0.81 & $(0.53-1.23)$ \\
\hline T1 & 30.0 & 25069 & 94 & 1.10 & $(0.83-1.47)$ & 1.13 & (0.83-1.53) \\
\hline $\mathrm{T} 2$ & 85.7 & 26282 & 88 & 0.98 & $(0.73-1.32)$ & 0.99 & $(0.74-1.34)$ \\
\hline T3 & 123.8 & 26373 & 90 & 1 (ref) & & 1 (ref) & \\
\hline$P_{\text {trend }}$ & & & & 0.69 & & 0.76 & \\
\hline Continuous $(50 \mathrm{~g} /$ & ake increment) & & & & & 1.02 & $(0.91-1.14)$ \\
\hline \multicolumn{8}{|c|}{ Fresh red meat $(\mathrm{g} / \text { day })^{\mathrm{c}}$} \\
\hline Non consumers & 0.0 & 13949 & 39 & 0.83 & $(0.57-1.21)$ & 0.90 & $(0.60-1.35)$ \\
\hline T1 & 25.0 & 24719 & 89 & 1.08 & $(0.80-1.44)$ & 1.16 & $(0.86-1.58)$ \\
\hline $\mathrm{T} 2$ & 71.2 & 26007 & 93 & 1.07 & $(0.80-1.43)$ & 1.10 & $(0.82-1.48)$ \\
\hline T3 & 106.8 & 25952 & 87 & 1 (ref) & & 1 (ref) & \\
\hline$P_{\text {trend }}$ & & & & 0.73 & & 0.97 & \\
\hline Continuous $(50 \mathrm{~g} /$ & ake increment) & & & & & 1.02 & $(0.91-1.15)$ \\
\hline \multicolumn{8}{|c|}{ Beef $(g / d a y)^{c}$} \\
\hline Non consumers & 0.0 & 2927 & 71 & 0.85 & $(0.62-1.17)$ & 0.82 & $(0.58-1.17)$ \\
\hline $\mathrm{T} 1$ & 6.3 & 22633 & 66 & 0.80 & $(0.58-1.11)$ & 0.84 & $(0.60-1.17)$ \\
\hline $\mathrm{T} 2$ & 19.3 & 23097 & 91 & 1.08 & $(0.80-1.46)$ & 1.12 & $(0.82-1.51)$ \\
\hline T3 & 43.3 & 21970 & 80 & 1 (ref) & & 1 (ref) & \\
\hline$P_{\text {trend }}$ & & & & 0.12 & & 0.15 & \\
\hline Continuous $(50 \mathrm{~g} /$ & ake increment) & & & & & 1.07 & $(0.84-1.36)$ \\
\hline \multicolumn{8}{|c|}{ Pork (g/day) ${ }^{\mathrm{c}}$} \\
\hline Non consumers & 0.0 & 19649 & 58 & 0.91 & $(0.65-1.28)$ & 0.88 & $(0.59-1.33)$ \\
\hline T1 & 6.1 & 22973 & 84 & 1.13 & $(0.83-1.55)$ & 1.12 & $(0.81-1.56)$ \\
\hline $\mathrm{T} 2$ & 28.4 & 24118 & 89 & 1.14 & $(0.84-1.55)$ & 1.16 & $(0.85-1.58)$ \\
\hline T3 & 58.3 & 23887 & 77 & 1 (ref) & & 1 (ref) & \\
\hline$P_{\text {trend }}$ & & & & 0.69 & & 0.77 & \\
\hline Continuous $(50 \mathrm{~g} /$ & ake increment) & & & & & 0.98 & $(0.80-1.20)$ \\
\hline \multicolumn{8}{|c|}{ Minced meat $(\mathrm{g} / \text { day })^{\mathrm{c}}$} \\
\hline Non consumers & 0.0 & 24939 & 73 & 0.90 & $(0.65-1.24)$ & 0.85 & $(0.59-1.24)$ \\
\hline $\mathrm{T} 1$ & 5.5 & 21824 & 84 & 1.18 & $(0.86-1.62)$ & 1.18 & $(0.85-1.64)$ \\
\hline $\mathrm{T} 2$ & 14.0 & 22107 & 80 & 1.11 & $(0.81-1.53)$ & 1.12 & $(0.81-1.54)$ \\
\hline T3 & 29.0 & 21757 & 71 & 1 (ref) & & 1 (ref) & \\
\hline$P_{\text {trend }}$ & & & & 0.59 & & 0.59 & \\
\hline Continuous $(50 \mathrm{~g} /$ & ake increment) & & & & & 1.07 & $(0.73-1.55)$ \\
\hline \multicolumn{8}{|c|}{ Liver (g/day) $)^{c}$} \\
\hline Non consumers & 0.0 & 65971 & 214 & 0.85 & $(0.66-1.08)$ & 0.87 & $(0.68-1.13)$ \\
\hline Consumers & 3.3 & 24646 & 94 & 1 (ref) & & 1 (ref) & \\
\hline Continuous $(25 \mathrm{~g} /$ & ake increment) & & & & & 1.55 & $(0.77-3.12)$ \\
\hline \multicolumn{8}{|c|}{ Chicken ${ }^{c, d}$} \\
\hline Non consumers & 0.0 & 31484 & 111 & 1.08 & $(0.79-1.47)$ & 1.17 & $(0.84-1.61)$ \\
\hline C1 & 5.3 & 21204 & 77 & 1.12 & $(0.80-1.56)$ & 1.16 & $(0.83-1.62)$ \\
\hline $\mathrm{C} 2$ & 13.2 & 18584 & 57 & 0.94 & $(0.66-1.35)$ & 0.93 & (0.65-1.33) \\
\hline $\mathrm{C} 3$ & 22.8 & 19355 & 63 & 1 (ref) & & 1 (ref) & \\
\hline$P_{\text {trend }}$ & & & & 0.44 & & 0.20 & \\
\hline Continuous $(50 \mathrm{~g} /$ & ake increment) & & & & & 1.00 & $(0.67-1.45)$ \\
\hline \multicolumn{8}{|c|}{ Processed meat (g/day) } \\
\hline Non consumers & 0.0 & 27388 & 97 & 1.16 & $(0.84-1.59)$ & 1.30 & $(0.91-1.87)$ \\
\hline $\mathrm{T} 1$ & 2.3 & 21362 & 64 & 0.98 & $(0.69-1.39)$ & 1.04 & (0.73-1.49) \\
\hline $\mathrm{T} 2$ & 8.2 & 20913 & 83 & 1.30 & $(0.94-1.80)$ & 1.31 & $(0.94-1.82)$ \\
\hline T3 & 20.3 & 20963 & 64 & 1 (ref) & & 1 (ref) & \\
\hline$P_{\text {trend }}$ & & & & 0.76 & & 0.34 & \\
\hline Continuous $(25 \mathrm{~g} /$ & ake increment) & & & & & 0.81 & (0.61-1.08) \\
\hline
\end{tabular}




\begin{tabular}{|c|c|c|c|c|c|c|c|}
\hline \multirow[b]{2}{*}{ Food item } & \multirow[b]{2}{*}{ Median intake } & \multirow[b]{2}{*}{ PY } & \multicolumn{5}{|c|}{ Breast Cancer } \\
\hline & & & Cases & $\mathrm{HR}$ & $(95 \% \mathrm{Cl})^{\mathrm{a}}$ & $\mathrm{HR}$ & $(95 \% \mathrm{Cl})^{b}$ \\
\hline \multicolumn{8}{|l|}{ Fish $^{\mathrm{e}}$} \\
\hline Non consumers & 0.0 & 31075 & 100 & 1.01 & $(0.71-1.42)$ & 1.11 & $(0.78-1.58)$ \\
\hline C1 & 4.6 & 19647 & 71 & 1.15 & $(0.80-1.66)$ & 1.21 & $(0.84-1.75)$ \\
\hline C2 & 14.8 & 24848 & 88 & 1.11 & $(0.78-1.58)$ & 1.18 & $(0.82-1.67)$ \\
\hline C3 & 29.9 & 15047 & 48 & 1 (ref) & & 1 (ref) & \\
\hline$P_{\text {trend }}$ & & & & 0.87 & & 0.70 & \\
\hline \multicolumn{2}{|c|}{ Continuous ( $25 \mathrm{~g} /$ day intake increment) } & & & & & 0.98 & $(0.82-1.16)$ \\
\hline
\end{tabular}

All of the women were presumed to be postmenopausal; PY (Person years at risk)

${ }^{\text {a }}$ Adjusted for age (y)

${ }^{\mathrm{b}}$ Adjusted for age (y), total energy intake (kcal), cigarette smoking (never, ever, current), alcohol consumption (g/day), BMI $\left(\mathrm{kg} / \mathrm{m}^{2}\right)$, non-occupational physical activity $(<30,30-60,60-90,>90 \mathrm{~min} / \mathrm{d})$, level of education (lower vocational, second and medium vocational, university and higher vocational), family history of breast cancer (yes/no), age menarche (y), age menopause $(<50, \geq 50$, unknown), age first child (y), hormone replacement therapy (yes/no), use of oral contraceptives (yes/no), and number of children.

${ }^{c}$ Intake based on raw meat weight.

${ }^{d}$ Categories of intake $0,>0-<6.6, \geq 6.6-<22.8$, and $\geq 22.8 \mathrm{~g} /$ day.

${ }^{\mathrm{e}}$ Categories of intake: $0,>0-<10, \geq 10-<20$, and $\geq 20 \mathrm{~g} /$ day.

\section{DISCUSSION}

Results from this prospective cohort study showed that, in age- and sex- adjusted models, vegetarians and pescetarians were at a reduced risk of lung cancer compared to individuals consuming meat on a daily basis. This effect disappeared after taking confounders, especially smoking, into account. We did not observe an association between meat consumption group and the risk of female breast and overall prostate cancer, whereas individuals consuming meat $\leq 1$ for day/week were at an increased risk of advanced prostate cancer compared to 6-7 days/week meat consumers.

Although non-meat consumers (vegetarians and pescetarians) were at a $>50 \%$ lower risk for lung cancer, this was largely the result of their lower prevalence of smoking. Comparable null findings after multivariable adjustment were previously observed [11-13]. Fruits and vegetables have also previously been reported to reduce lung cancer risk $[18,19]$ and our results suggest that these factors indeed explain some of the observed risk reduction among vegetarians (up to 18 and 5\%, respectively). However, given that smoking is such a strong risk factor for lung cancer, these latter observations were likely due to residual confounding by smoking; when we corrected for smoking the effect of fruits and vegetables disappeared (results not shown)

Our null findings regarding post-menopausal breast cancer risk are in line with other prospective studies comparing vegetarians to non-vegetarians and with a pooled analysis of five cohort studies on breast cancer mortality. In contrast, the UK Women's Cohort Study reported a lower post-menopausal breast cancer risk among non-meat consumers compared to high meat consumers [14] although this was not observed in their dietary pattern analyses [15]. Vegetarian diets are rich in fiber and soy. Fiber was associated with a reduced risk of breast cancer in a meta-analysis of prospective studies [20] and soy contains isoflavones, which have previously been associated with a significant reduced risk of post-menopausal breast cancer in Asian populations [21]. 


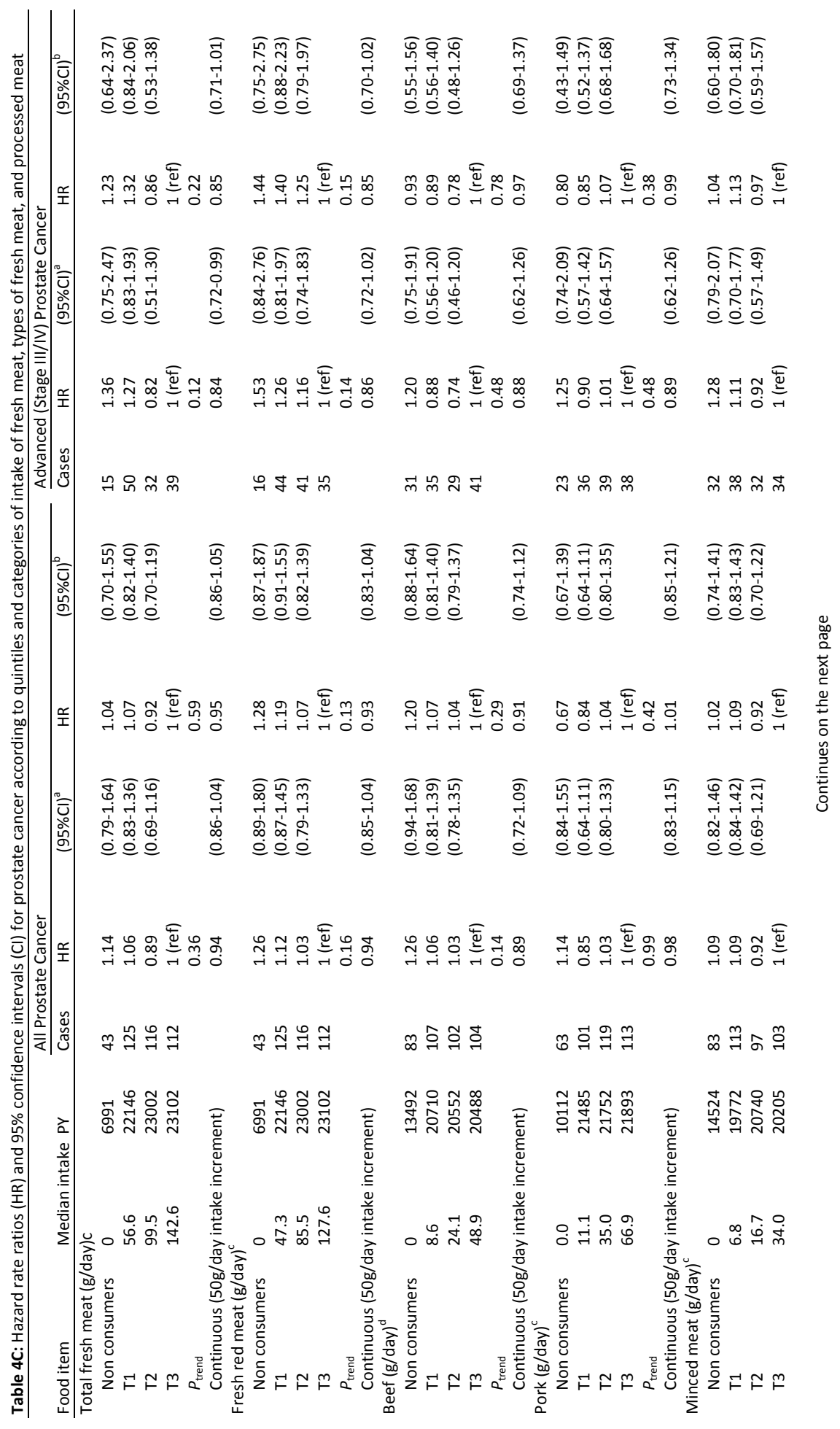




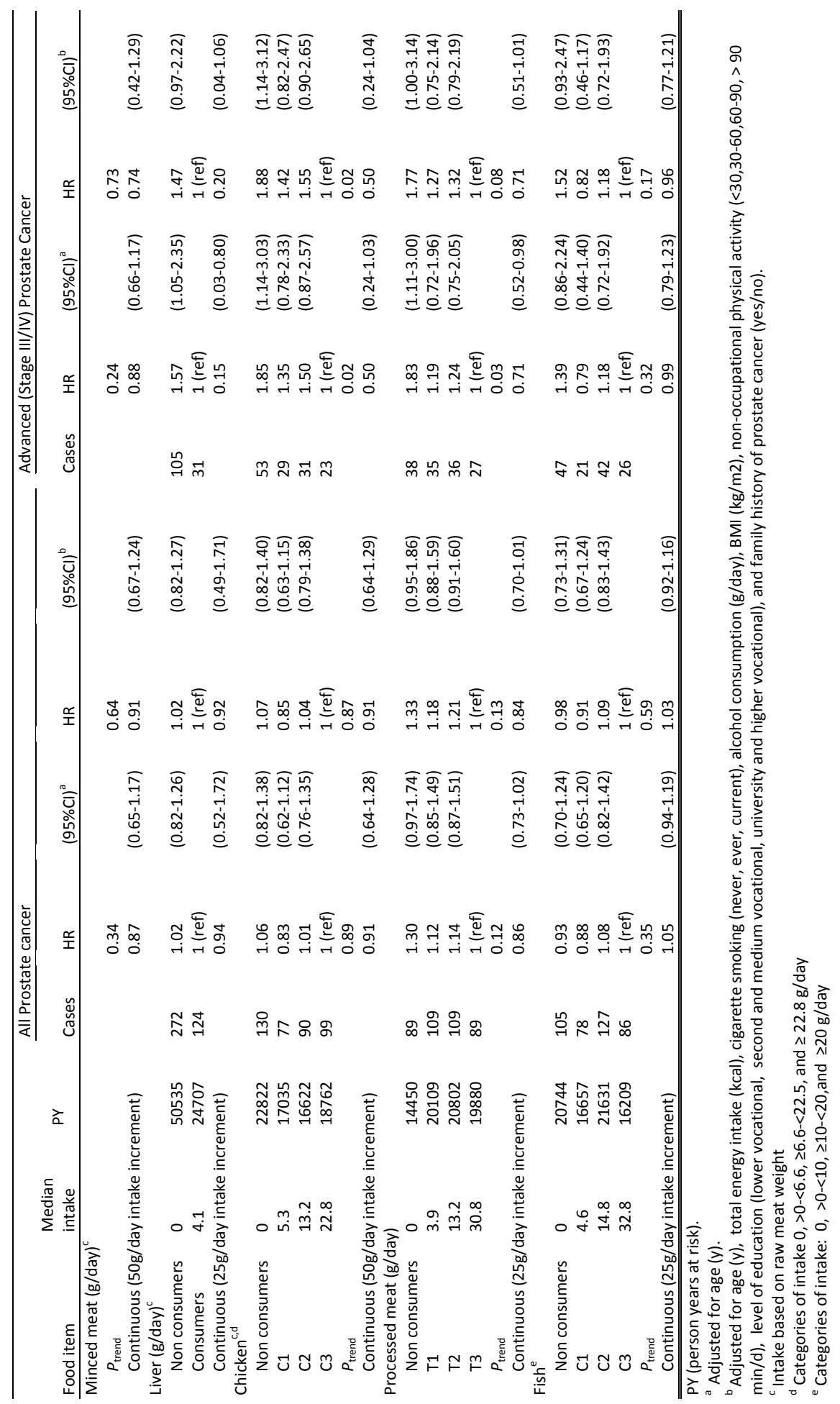


However, compared to the average soy intake in four Asian countries (ranging from 38 $134 \mathrm{gr} /$ day [22]), the soy product intake among vegetarians in our population was likely too low to exert an effect ( $\sim 15 \mathrm{gr} /$ day). The vegetarians in our population were not at an increased risk for breast cancer based on their reproductive and hormonal characteristics [1]. However, compared to individuals that consume meat on a daily basis, vegetarians had lower levels of alcohol consumption, a lower BMI, and higher levels of physical activity [1], which are all associated with a lower risk of breast cancer. We had too little statistical power to stratify our analyses by hormone receptor status of the tumor since this information was only available for $45 \%$ of all cases.

Contrary to our hypothesis, we observed a statistically significant increased risk of advanced, but not overall, prostate cancer for 1 day/week meat consumers compared to those with the highest meat intake (6/7 days/week). A similar, though non-significant, increased risk was found for vegetarians and pescetarians. In contrast, vegetarians were at lower risk of overall prostate cancer in the AHS I [11] after adjustment for age, but this may result from differences in PSA screening practices [10]. Although both the vegetarians and meat consumers in the AHS were generally non- smokers and non-alcohol users, these results were not reproduced by others who also corrected for additional lifestyle factors $[12,13]$.

We are the first to investigate vegetarianism and low meat consumption in prostate cancer subgroups based on disease stage, and provide further evidence that risk factors for prostate cancer may indeed differ for advanced and non-advanced tumors [16]. The increased risk of advanced prostate cancer for the non- and low meat consumers could not be explained by other dietary and non-dietary factors than the frequency of meat consumption. When further examining meat subgroups, we found inverse associations with chicken, processed meat, and liver but not for all other meat items, which contrasts previous studies that generally reported positive or null associations between advanced prostate cancer and meat and meat carcinogens (e.g. ref $[23,24])$. As a result, we are careful interpreting these findings and future studies, with a larger number of advanced prostate cancer cases, vegetarians and low meat consumers, should replicate our findings.

The incidence of female breast and prostate cancers increased after the introduction of screening $[25,26]$. Although we previously reported a lower breast cancer screening mammography rate among vegetarians (23\%) compared to the highest meat consumers (29\%)[1], correcting for these screening practices did not change our risk estimates noticeably. A recent investigation in the AHS II, showed that vegetarians are also less likely to follow prostate cancer screening guidelines compared to non-vegetarians [10]. However, PSA testing became more common in the Netherlands after 1990 [27] and is not propagated as a screening tool. Whether PSA testing could be responsible for the differences in risk estimates for advanced prostate cancer between the meat consumption groups is questionable.

Strengths of our study include the prospective design, the long, nearly complete follow-up, and detailed information on diet and potential risk factors of cancer. Although our analyses have been performed using baseline FFQ data only, the validity of the FFQ has been tested and shown to be representative for dietary habits over a period of at least 5 years [28]. 
In conclusion, vegetarians, pescetarians and low meat consumers did not have a reduced risk of lung, female breast and overall prostate cancer compared to individuals consuming meat on a daily basis after taking confounders, especially smoking, into account. Our observation that $\leq 1$ day/week meat consumption was associated with increased risk of advanced prostate cancer may be due to chance and warrants investigation on other studies.

\section{REFERENCES}

1. Gilsing, A.M., et al., The Netherlands Cohort Study-Meat Investigation Cohort; a population-based cohort over-represented with vegetarians, pescetarians and low meat consumers. Nutr J, 2013. 12: p. 156.

2. Haddad, E.H. and J.S. Tanzman, What do vegetarians in the United States eat? Am J Clin Nutr, 2003. 78(3 Suppl): p. 626S-632S.

3. Doll, R. and R. Peto, The causes of cancer: quantitative estimates of avoidable risks of cancer in the United States today. J Natl Cancer Inst, 1981. 66(6): p. 1191-308.

4. World Cancer Research Fund/American Institute for Cancer Research, Food, Nutrition, Physical Activity, and the Prevention of Cancer: a Global Perspective. 2007, AICR: Washington DC.

5. Gilsing, A.M., et al., Vegetarianism, low meat consumption and the risk of colorectal cancer in a population based cohort study. Submitted for publication.

6. Alexander, D.D., et al., A review and meta-analysis of prospective studies of red and processed meat intake and prostate cancer. Nutr J, 2010. 9: p. 50.

7. Alexander, D.D., et al., A review and meta-analysis of red and processed meat consumption and breast cancer. Nutr Res Rev, 2010. 23(2): p. 349-65.

8. Yang, W.S., et al., Meat consumption and risk of lung cancer: evidence from observational studies. Ann Oncol, 2012. 23(12): p. 3163-70.

9. Chan, J.M., P.H. Gann, and E.L. Giovannucci, Role of diet in prostate cancer development and progression. J Clin Oncol, 2005. 23(32): p. 8152-60.

10. Ibrayev, Y., et al., Utilization of prostate cancer screening according to dietary patterns and other demographic variables. The adventist health study-2. J Cancer, 2013. 4(5): p. 416-26.

11. Fraser, G.E., Associations between diet and cancer, ischemic heart disease, and all-cause mortality in non-Hispanic white California Seventh-day Adventists. Am J Clin Nutr, 1999. 70(3 Suppl): p. 532S-538S.

12. Key, T.J., et al., Cancer incidence in British vegetarians. Br J Cancer, 2009. 101(1): p. 192-7.

13. Key, T.J., et al., Cancer incidence in vegetarians: results from the European Prospective Investigation into Cancer and Nutrition (EPIC-Oxford). Am J Clin Nutr, 2009. 89(5): p. 1620S-1626S.

14. Taylor, E.F., et al., Meat consumption and risk of breast cancer in the UK Women's Cohort Study. Br J Cancer, 2007. 96(7): p. 1139-46.

15. Cade, J.E., et al., Common dietary patterns and risk of breast cancer: analysis from the United Kingdom Women's Cohort Study. Nutr Cancer, 2010. 62(3): p. 300-6.

16. Giovannucci, E., et al., Risk factors for prostate cancer incidence and progression in the health professionals follow-up study. Int J Cancer, 2007. 121(7): p. 1571-8.

17. van den Brandt, P.A., et al., A large-scale prospective cohort study on diet and cancer in The Netherlands. J Clin Epidemiol, 1990. 43(3): p. 285-95.

18. Buchner, F.L., et al., Fruits and vegetables consumption and the risk of histological subtypes of lung cancer in the European Prospective Investigation into Cancer and Nutrition (EPIC). Cancer Causes Control, 2010. 21(3): p. 357-71.

19. Smith-Warner, S.A., et al., Fruits, vegetables and lung cancer: a pooled analysis of cohort studies. Int J Cancer, 2003. 107(6): p. 1001-11.

20. Aune, D., et al., Dietary fiber and breast cancer risk: a systematic review and meta-analysis of prospective studies. Ann Oncol, 2012. 23(6): p. 1394-402.

21. Dong, J.Y. and L.Q. Qin, Soy isoflavones consumption and risk of breast cancer incidence or recurrence: a meta-analysis of prospective studies. Breast Cancer Res Treat, 2011. 125(2): p. 315-23. 
22. Messina, M., C. Nagata, and A.H. Wu, Estimated Asian adult soy protein and isoflavone intakes. Nutr Cancer, 2006. 55(1): p. 1-12.

23. Cross, A.J., et al., A prospective study of meat and meat mutagens and prostate cancer risk. Cancer Res, 2005. 65(24): p. 11779-84.

24. Sinha, R., et al., Meat and meat-related compounds and risk of prostate cancer in a large prospective cohort study in the United States. Am J Epidemiol, 2009. 170(9): p. 1165-77.

25. Welch, H.G. and P.C. Albertsen, Prostate cancer diagnosis and treatment after the introduction of prostate-specific antigen screening: 1986-2005. J Natl Cancer Inst, 2009. 101(19): p. 1325-9.

26. Bleyer, A. and H.G. Welch, Effect of three decades of screening mammography on breast-cancer incidence. N Engl J Med, 2012. 367(21): p. 1998-2005.

27. Post, P.N., et al., Trends in incidence and mortality rates for prostate cancer before and after prostatespecific antigen introduction. A registry-based study in southeastern Netherlands, 1971-1995. Eur J Cancer, 1998. 34(5): p. 705-9.

28. Goldbohm, R.A., et al., Reproducibility of a food frequency questionnaire and stability of dietary habits determined from five annually repeated measurements. Eur J Clin Nutr, 1995. 49(6): p. 420-9. 


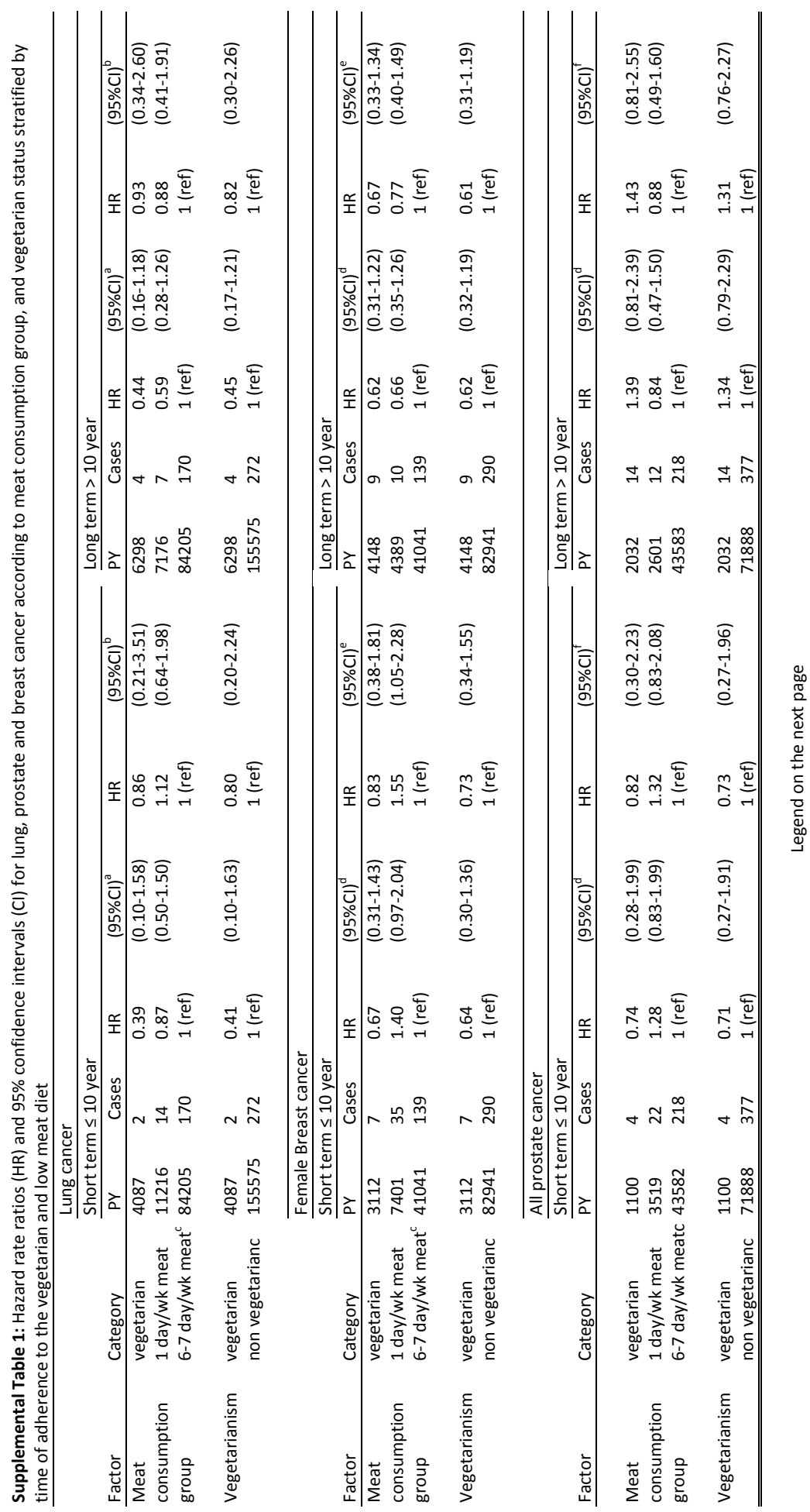




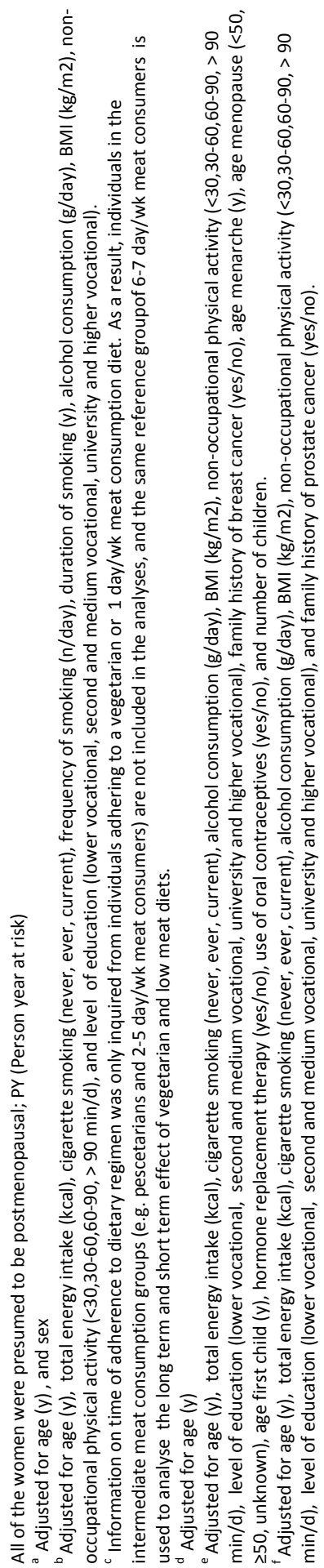





\title{
CHAPTER 7
}

\section{Consumption of dietary fat and meat and risk of ovarian cancer in the Netherlands Cohort Study}

\author{
Anne M.J. Gilsing \\ Matty P. Weijenberg \\ R. Alexandra Goldbohm \\ Piet A. van den Brandt \\ and Leo J. Schouten
}

American Journal of Clinical Nutrition 2011;93(1):118-26 


\section{ABSTRACT}

Background: Evidence linking dietary factors to ovarian cancer is conflicting but several epidemiological studies have suggested that consumption of dietary fat and meat may increase the risk of ovarian cancer.

Objective: We studied the association of intake of total fat, sources and subtypes of fat, fresh meat, processed meat and fish with ovarian cancer risk within the Netherlands Cohort Study (NLCS).

Methods: The NLCS includes 62573 postmenopausal women, aged 55-69 y at baseline, who completed a baseline questionnaire on dietary habits and other risk factors for cancer in 1986. After 16.3 years of follow-up, 340 ovarian cancer cases and 2161 subcohort members were available for case-cohort analysis. Multivariable rate ratios (RR) were adjusted for age at baseline, total energy intake, oral contraceptive use, and parity.

Results: No clear association between intake of total fat, saturated fat, mono- and polyunsaturated fats, animal fat, plant based fat, dairy fat, other fat sources, fresh meat, processed meat and fish and ovarian cancer risk was observed. Consumption of transunsaturated fatty acids was positively associated with ovarian cancer risk. The multivariable RR for women in the highest compared to the lowest quintile of intake was 1.51 (95\%confidence interval, 1.04, 2.20; P-trend=0.01). Although no statistically significant interactions by oral contraceptive use or parity were found, effect sizes were generally more pronounced and significant in never pill users and parous women.

Conclusion: This prospective study suggests that trans-unsaturated fatty acids, but no other types of fat or meat, are associated with increased ovarian cancer risk. 


\section{INTRODUCTION}

Research suggests that the etiology of ovarian cancer is predominantly related to hormones and reproduction; the strongest known protective factors being oral contraceptive use and parity [1, 2]. In contrast, evidence linking dietary factors to ovarian cancer is inconsistent and limited although several epidemiological studies have suggested that consumption of dietary fat and meat may increase the risk of ovarian cancer.

Ecologic and migrant studies were the first to provide epidemiological support for a direct association between the intake of dietary fat, particularly animal fat, and meat and ovarian cancer mortality [3,4]. Since then, numerous case control [5-17] but only a few prospective cohort studies [18-22] have published on total, animal or saturated fat intake, or meat consumption and ovarian carcinogenesis at the individual level. Nonetheless, a recent Expert Panel Report concluded that these epidemiological data were either of too low quality, too inconsistent or the number of studies too few to allow conclusions to be reached [23].

Dietary fat and meat are hypothesized to affect ovarian carcinogenesis primarily via hormone related mechanisms. High intake of dietary fat is suggested to expose the ovarian epithelium to high concentrations of endogenous circulating estrogens which may, through cell- damage and proliferation, increase the likelihood of cancer development [24-26]. Although this mechanism is not supported by all studies [27-29], a meta-analysis of dietary intervention studies to lower total fat intake observed significant reductions in serum estradiol levels [30] and research showed higher urinary estrogen excretion levels in omnivore compared to vegetarian post-menopausal women [31]. Likewise, meat consumption is suggested to increase ovarian carcinogenesis either via its relatively high fat content or alternatively through the cancerous effect of meat specific mutagens; N-nitroso compounds, heterocyclic amines and polycyclic aromatic hydrocarbons can be derived from natural food or during the process of food preservation and preparation [32].

Nevertheless, little is known concerning the association of ovarian cancer risk and different sources and types of fat and meat. Therefore, the objective of the present study was to test the hypothesis that dietary intake of fat and specific sources of fat, and intakes of fresh meat, processed meat and fish is associated with the subsequent risk of ovarian cancer among postmenopausal women in a large prospective cohort study including extensive quantitative food frequency and lifestyle information and a large number of ovarian cancer cases in the Netherlands. Because the effect of fat and meat intake may vary according to parity and oral contraceptive use we also examined whether the associations differed by these well-established protective factors of ovarian cancer.

\section{METHODS}

\section{Study population and cancer follow-up}

The Netherlands Cohort Study was initiated in September 1986 and includes 62573 women aged 55-69 years at baseline, originating from 204 municipalities with computerized population registries. Full details of the study design have been described elsewhere [33]. At the start of the study, participants completed a self-administered questionnaire on 
dietary habits, lifestyle characteristics, medical history, and other potential risk factors for cancer. The case-cohort approach was used for reasons of efficiency in questionnaire processing and follow-up. Case subjects were enumerated from the entire cohort, whereas the accumulated person years of the entire cohort were estimated from a random subcohort of 2589 women, chosen immediately after baseline. The entire cohort is being monitored for cancer occurrence by annual record linkage to the Netherlands Cancer Registry and the Netherlands Pathology, together providing a near $100 \%$ coverage [34]. None of the female subcohort members were lost to follow-up.

For cases and subcohort members, all prevalent cancer cases at baseline other than nonmelanoma skin cancer were excluded. After 16.3 years of follow-up (until December 2002) 421 incident, microscopically confirmed, primary ovarian carcinomas were detected. Following the exclusion of non-epithelial tumors $(n=13)$, borderline invasive tumors $(n=$ 14), and cases with incomplete or inconsistent dietary data [35] ( $n=42), 352$ cases remained eligible for analyses. In the subcohort, women who had reported at baseline to have undergone an oophorectomy $(n=32)$, and women with incomplete or inconsistent dietary data $(n=190)$ were excluded, leaving 2216 subcohort members (including 16 ovarian cancer cases) for analysis. The NLCS has been approved by the institutional review boards of the TNO Quality of Life Research Institute (Zeist, the Netherlands) and Maastricht University (Maastricht, the Netherlands).

\section{Questionnaire}

All participants completed a 150 item semi-quantitative food-frequency questionnaire (FFQ) at baseline, estimating the average frequency and amounts of foods and beverages consumed over the previous 12 months. Daily mean nutrient intakes were calculated from the FFQ dietary data by summing the multiplied frequencies and number of serving sizes of all food items with their tabulated nutrient contents from the Dutch food composition table [36].

Intake of specific fatty acids was based on a separate database with specific fatty acids derived from the TRANSFAIR study [37]. In the database, total fat included triglycerides and other lipids, such as phospholipids and sterols. The percentage of triglycerides in total fat is assumed to be $93 \%$ on average, but it varies across food sources. The concentrations of fatty acids were based on the concentrations before 1995, when changes in trans- content of manufactured products, such as margarines, led to a substantial decrease in the intake of trans unsaturated fatty acids (TFA). Coding of fresh meat items was based on raw weight to take into account the amount of fat originally present in the meat but eventually ending up in the gravy, which is usually consumed as well. Processed meat was defined as meat items that had undergone some form of preservation (mostly treatment with nitrate salt, sometimes smoked or fermented).

The FFQ has been validated and tested for reproducibility [35, 38]. Crude (energy and gender adjusted between brackets) Pearson correlation coefficients ( $r 2$ ) between the questionnaire and the 9-day diet record (kept over 3-day periods, 4-5 months apart) were 0.74 for energy, $0.72(0.52)$ for total fat, $0.73(0.58)$ for saturated fatty acids (SFA) and 0.73 (0.75) for poly unsaturated fatty acids (PUFA). The Spearman correlation coefficients for fresh meat, processed meat, and fish were $0.46,0.54$ and 0.53 respectively [35]. 


\section{Statistical analyses}

The comprehensive set of exposure variables in our analyses included the intake of the following nutrients (in g/day): total fat, fat from plant foods, fat from animal products (milk, eggs, meat and fish), ratio of plant based to animal fat, fat from meat, dairy fat, fat from margarines, SFA, mono unsaturated fatty acids (MUFA), PUFA, TFA.

Fat from plant foods covers fat naturally present in all plant foods (including vegetable oils), but exclude (partly hydrogenated) vegetable fat from margarine. Fat from meat refers to fat from fresh meats and fat from processed meats. Fat from fish was not analyzed separately because fish intake was measured with limited accuracy only (no differentiation oily- vs. whitefish), whereas fat from margarines was assessed comprehensively in the NLCS. We intentionally included TFAs in analyses; these are characterized by part hydrogenation of plant based oils which are hypothesized to have distinct carcinogenic effects. Intakes of fat items were all adjusted for energy intake by the residual method [39]. Also the following food groups and foods were selected (in g/day): fresh meat (beef, pork, minced meat, chicken, liver), processed meat, fish, fresh red meat (fresh meat without chicken) and beef, pork, minced meat, chicken, and liver as separate items.

Age and energy adjusted and multi-variable adjusted incidence rate ratios (RRs) and their corresponding $95 \%$ confidence intervals $(95 \% \mathrm{Cl}$ ) were estimated using Cox proportional hazards models. The total person-years at risk, estimated from the subcohort, were used in analyses [40]. Standard errors were estimated using the robust Huber-White sandwich estimator to account for additional variance introduced by sampling from the cohort [41]. The proportional hazards assumption was tested using the scaled Schoenfeld residuals; in case of violation the follow-up period was stratified into 3 categories $(<5 ; 5-10 ; \geq 10$ years of follow-up) and a test for interaction between the determinant and time was calculated and time-stratified results were estimated.

The covariates included in the multivariate analyses were either a priori selected risk factors of ovarian cancer or variables that changed the risk estimates for total fat or total fresh meat intake by $10 \%$ or more. The latter criterion was not met for any other than the predefined covariates resulting in a final model including age $(\mathrm{y})$, use of oral contraceptives (ever versus never), and number of children (n). Total energy intake (kcal/day) was included in both the age and the multivariable-adjusted model. RRs for energy-adjusted total fat and fatty acids can be interpreted as the effect of an increase in these variables relative to a decrease of an equivalent amount of energy from other energy delivering nutrients (i.e., substituting these exposure nutrients for other energy delivering nutrients). In addition, to assess the independent contribution of SFA, MUFA, PUFA TFA total fat intake was also included in the respective multivariable adjusted models. To further explore our data, we ran an additional model including all fatty acids together (SF, MUFA, PUFA, and TFA) to investigate the relative contribution of each fatty acid. The independent contribution of the individual meat categories (total fresh meat, fresh red meat, beef, pork, minced meat, chicken, and liver) was examined, by creating a model in which the complementary meat items were also included in the respective multivariable adjusted models.

Subjects were classified into quintiles of consumption (with the lowest quintile regarded as the reference group), and as continuous variables. The latter were reported in one 
standard deviation increase of consumption for the fat and fatty acid items, an increase of 0.2 in the ratio of plant based to animal fat, and $25 \mathrm{~g} /$ day increase for all meat items and for fish. For some variables, categories were used instead of quintiles. For liver intake, there was a non-user and a user group ( $>0 \mathrm{~g} / \mathrm{day}$ ). For both chicken and fish a non-user and 3 user categories $(0-<13.2, \geq 13.2-<22.8$ and $\geq 22.8 \mathrm{~g} /$ day for chicken; $0-<10, \geq 10-<20$ and $\geq$ $20 \mathrm{~g} /$ day for fish) were defined.

To enable comparison, the age and energy-adjusted analyses were restricted to subjects included in multivariable-adjusted analyses (e.g., with no missing values on confounding variables), leaving 2161 subcohort members and 340 ovarian cancer cases for analyses. For each analysis, trends were evaluated with the Wald test by assigning participants the median value for each level of the categorical exposure variables among the subcohort members and this variable was entered as a continuous term in the Cox regression model.

Interaction of meat and fat intake by use of oral contraceptives or parity was tested using cross-product terms between continuous variables of meat or fat intake on one hand and oral contraceptive use and parity as a dichotomous variable (categories being ever use and never use; nulliparous ( 0 children) and parous ( $\geq 1$ children), respectively) on the other hand.

All analyses were performed using STATA Statistical Software (Intercooled STATA, version 10; Stata-Corp LP, College Station, TX). All tests were two-tailed and differences were regarded as statistically significant at $P<0.05$.

\section{RESULTS}

After 16.3 years of follow-up of 62573 women, 394 invasive epithelial ovarian cancer cases occurred, 340 of which were in women with complete and consistent dietary data. Table 1 lists the baseline characteristics for cases and subcohort members of which the greater part did not differ between ovarian cancer cases and subcohort members. However, the percentage of women who reported ever use of oral contraceptives and the percentage of current smokers was higher among subcohort members than among cases. Fewer cases reported to have undergone a hysterectomy and cases had a smaller number of children than subcohort members. No striking differences between cases and subcohort members were observed regarding baseline dietary intakes of the exposures of interest.

No association was found when examining the relation between the risk of ovarian cancer and the dietary intake of total fat, fat from meat, fat from animal products, fat from margarines, SFA, MUFA, PUFA, in the multivariable model (Table 2). Regarding fat from plant foods, an inverse association was found, showing a statistically significant decreased risk of ovarian cancer for all quintiles compared to the lowest quintile (multivariable RRs = $1.00,0.58,0.67,0.67$ and 0.64 , respectively, $P$-trend $=0.05$ ) (Table 2). A statistically significant increased ovarian cancer risk per 1 SD intake of dairy fat intake as a continuous variable was observed (multivariable $\mathrm{RR}=1.13,95 \% \mathrm{Cl}$ : $1.01,1.27$ ), but no evidence of a linear trend was found (Table 2). 
Table 1:Baseline characteristics (means or percent) and dietary intakes of exposures of interest of ovarian cancer cases and subcohort members, NLCS 1986-2002

\begin{tabular}{|c|c|c|c|c|c|c|}
\hline \multirow{2}{*}{$\begin{array}{l}\text { Characteristics } \\
\mathrm{N}\end{array}$} & \multicolumn{3}{|c|}{ Ovarian cancer cases } & \multicolumn{3}{|c|}{ Subcohort } \\
\hline & 340 & & & 2161 & & \\
\hline Age (y) & 61.8 & \pm & 4.31 & 61.4 & \pm & 4.3 \\
\hline Current smokers (\%) & 17.6 & & & 21.2 & & \\
\hline Years of smoking $(y)$ & 26.8 & \pm & 12.4 & 27.8 & \pm & 12.5 \\
\hline $\mathrm{BMI}\left(\mathrm{kg} / \mathrm{m}^{2}\right)$ & 25.1 & \pm & 3.5 & 25.1 & \pm & 3.5 \\
\hline \multicolumn{7}{|l|}{ Physical activity (non-occupational) (\%) } \\
\hline$<30 \mathrm{~min} /$ day & 27.1 & & & 24.2 & & \\
\hline $30-60 \mathrm{~min} /$ day & 31.5 & & & 30.7 & & \\
\hline $60-90 \mathrm{~min} / \mathrm{day}$ & 23.2 & & & 22.6 & & \\
\hline$>90 \mathrm{~min} /$ day & 17.1 & & & 21.2 & & \\
\hline \multicolumn{7}{|l|}{ Level of education (\%) } \\
\hline Low & 58.8 & & & 56.7 & & \\
\hline Medium & 33.2 & & & 34.5 & & \\
\hline High & 8.0 & & & 8.8 & & \\
\hline \multicolumn{7}{|l|}{ Reproductive and hormonal factors } \\
\hline Age at menarche $(y)$ & 13.7 & \pm & 1.8 & 13.7 & \pm & 1.8 \\
\hline Age at menopause (y) & 49.2 & \pm & 4.0 & 48.7 & \pm & 4.5 \\
\hline Use of oral contraceptives (\% ever) & 18.5 & & & 24.9 & & \\
\hline Hysterectomy (\% yes) & 7.4 & & & 14.2 & & \\
\hline Use of post-menopausal hormones (\% ever) & 12.1 & & & 12.4 & & \\
\hline \multicolumn{7}{|l|}{ Parity (\%) } \\
\hline 0 children & 23.2 & & & 18.0 & & \\
\hline 1 child & 9.7 & & & 8.2 & & \\
\hline 2 children & 25.6 & & & 21.7 & & \\
\hline$>2$ children & 41.5 & & & 52.1 & & \\
\hline Age at first birth (y) & 21.1 & \pm & 12.2 & 22.1 & \pm & 11.0 \\
\hline Family history of ovarian cancer (\%) & 0.6 & & & 0.0 & & \\
\hline Family history of breast cancer (\%) & 7.9 & & & 8.7 & & \\
\hline \multicolumn{7}{|l|}{ Dietary factors, daily intake (g) } \\
\hline \multicolumn{7}{|l|}{ Total fat and specific fatty acids 2} \\
\hline Total fat & 74.1 & \pm & 9.9 & 73.9 & \pm & 10.3 \\
\hline Fat from plant based products & 8.5 & \pm & 6.4 & 9.0 & \pm & 6.8 \\
\hline Fat from animal sources, total & 40.1 & \pm & 13.6 & 38.6 & \pm & 13.1 \\
\hline Fat from meat fat & 15.6 & \pm & 6.6 & 15.9 & \pm & 7.1 \\
\hline Fat from dairy products & 20.4 & \pm & 13.7 & 18.5 & \pm & 12.6 \\
\hline Fat from margarines & 25.5 & \pm & 13.0 & 26.3 & \pm & 13.2 \\
\hline Saturated fatty acids & 30.6 & \pm & 6.3 & 29.7 & \pm & 5.8 \\
\hline Monounsaturated fatty acids & 27.5 & \pm & 4.8 & 27.5 & \pm & 5.0 \\
\hline Polyunsaturated fatty acids & 14.5 & \pm & 6.3 & 15.0 & \pm & 6.1 \\
\hline trans Unsaturated fatty acids & 2.6 & \pm & 1.0 & 2.5 & \pm & 0.9 \\
\hline \multicolumn{7}{|c|}{ Types of fresh meat 3 , processed meat, fish and eggs } \\
\hline Total fresh meat & 92.0 & \pm & 34.4 & 93.4 & \pm & 40.0 \\
\hline Fresh red meat & 80.0 & \pm & 33.4 & 80.7 & \pm & 38.6 \\
\hline Beef & 26.0 & \pm & 21.6 & 24.2 & \pm & 21.7 \\
\hline Pork & 34.0 & \pm & 24.6 & 35.5 & \pm & 28.4 \\
\hline Minced meat & 16.0 & \pm & 14.2 & 16.9 & \pm & 15.5 \\
\hline Liver & 1.7 & \pm & 4.1 & 1.7 & \pm & 4.0 \\
\hline Chicken & 12.9 & \pm & 14.2 & 13.4 & \pm & 15.6 \\
\hline Other meat (e.g. horsemeat, lamb) & 2.2 & \pm & 6.5 & 2.4 & \pm & 7.0 \\
\hline Processed meat & 10.4 & \pm & 11.3 & 10.5 & \pm & 11.8 \\
\hline Fish & 10.9 & \pm & 11.8 & 11.7 & \pm & 13.5 \\
\hline Eggs & 15.3 & \pm & 10.3 & 14.8 & \pm & 9.8 \\
\hline
\end{tabular}

1 Mean \pm SD (all such values)

2 Energy-adjusted intake

3 Intake based on raw meat weight 
Table 2: Relative risks (RR) and 95\% confidence intervals (CI) for ovarian cancer according to quintiles of intake of total fat - fat sources and specific fatty acids - NLCS 1986-2002 ${ }^{1}$

\begin{tabular}{|c|c|c|c|c|c|c|c|}
\hline \multirow[b]{2}{*}{ Food item } & \multirow[b]{2}{*}{$\begin{array}{l}\text { Median } \\
\text { intake }^{2}\end{array}$} & \multirow[b]{2}{*}{ PY } & \multicolumn{5}{|c|}{ Total ovarian cancer cases } \\
\hline & & & Cases & $\mathrm{RR}^{3}$ & $(95 \% \mathrm{Cl})$ & $\mathrm{RR}^{4}$ & $(95 \% \mathrm{Cl})$ \\
\hline \multicolumn{8}{|l|}{ Total fat ${ }^{5}$ (g/day) } \\
\hline $1^{6}$ & 61.0 & 6399 & 70 & 1.00 & & 1.00 & \\
\hline 2 & 69.1 & 6462 & 60 & 0.86 & $(0.59-1.24)$ & 0.86 & $(0.59-1.25)$ \\
\hline 3 & 73.9 & 6388 & 74 & 1.08 & $(0.75-1.54)$ & 1.07 & $(0.75-1.53)$ \\
\hline 4 & 78.9 & 6408 & 61 & 0.88 & (0.61-1.27) & 0.87 & $(0.60-1.26)$ \\
\hline 5 & 86.5 & 6466 & 75 & 1.06 & $(0.74-1.50)$ & 1.04 & $(0.73-1.49)$ \\
\hline$P_{\text {trend }}$ & & & & 0.73 & & 0.80 & \\
\hline Continuous 1 SD & & & & 1.02 & $(0.91-1.13)$ & 1.01 & $(0.90-1.13)$ \\
\hline \multicolumn{8}{|c|}{ Fat from plant sources ${ }^{5}$ (g/day) } \\
\hline $1^{6}$ & 2.8 & 6324 & 95 & 1.00 & & 1.00 & \\
\hline 2 & 5.8 & 6495 & 56 & 0.57 & $(0.40-0.83)$ & 0.58 & $(0.40-0.84)$ \\
\hline 3 & 8.0 & 6365 & 64 & 0.67 & $(0.46-0.96)$ & 0.67 & $(0.46-0.96)$ \\
\hline 4 & 10.6 & 6421 & 64 & 0.68 & $(0.47-0.97)$ & 0.67 & $(0.47-0.96)$ \\
\hline 5 & 15.9 & 6519 & 61 & 0.63 & $(0.45-0.90)$ & 0.64 & $(0.45-0.91)$ \\
\hline$P_{\text {trend }}$ & & & & 0.06 & & 0.05 & \\
\hline Continuous 1 SD & & & & 0.93 & $(0.81-1.06)$ & 0.93 & (0.81-1.07) \\
\hline \multicolumn{8}{|c|}{ Fat from animal sources ${ }^{5}$ (g/day) } \\
\hline $1^{6}$ & 23.9 & 6436 & 71 & 1.00 & & 1.00 & \\
\hline 2 & 31.4 & 6407 & 61 & 0.85 & $(0.59-1.23)$ & 0.84 & $(0.58-1.22)$ \\
\hline 3 & 36.7 & 6511 & 58 & 0.81 & (0.56-1.17) & 0.81 & $(0.56-1.18)$ \\
\hline 4 & 43.6 & 6442 & 56 & 0.78 & $(0.53-1.13)$ & 0.78 & $(0.53-1.13)$ \\
\hline 5 & 56.6 & 6327 & 94 & 1.31 & $(0.94-1.84)$ & 1.30 & $(0.93-1.83)$ \\
\hline$P_{\text {trend }}$ & & & & 0.19 & & 0.19 & \\
\hline Continuous 1 SD & & & & 1.12 & $(0.98-1.25)$ & 1.11 & $(0.99-1.25)$ \\
\hline \multicolumn{8}{|c|}{ Ratio plant based vs animal fat } \\
\hline $1^{6}$ & 0.06 & 6302 & 76 & 1.00 & & 1.00 & \\
\hline 2 & 0.13 & 6430 & 69 & 0.89 & $(0.63-1.27)$ & 0.92 & $(0.65-1.31)$ \\
\hline 3 & 0.20 & 6462 & 76 & 0.99 & $(0.70-1.40)$ & 1.00 & $(0.70-1.41)$ \\
\hline 4 & 0.30 & 6364 & 66 & 0.88 & $(0.61-1.26)$ & 0.90 & (0.63-1.29) \\
\hline 5 & 0.53 & 6550 & 53 & 0.69 & $(0.47-1.01)$ & 0.70 & $(0.48-1.02)$ \\
\hline$P_{\text {trend }}$ & & & & 0.08 & & 0.09 & \\
\hline Continuous 1 SD & & & & 0.95 & $(0.86-1.05)$ & 0.95 & $(0.86-1.06)$ \\
\hline \multicolumn{8}{|c|}{ Fat from fresh and processed meats ${ }^{5}$ (g/day) } \\
\hline $1^{6}$ & 7.6 & 6633 & 75 & 1.00 & & 1.00 & \\
\hline 2 & 12.2 & 6558 & 67 & 0.91 & $(0.64-1.31)$ & 0.93 & $(0.65-1.34)$ \\
\hline 3 & 15.2 & 6456 & 70 & 0.97 & $(0.68-1.38)$ & 0.96 & $(0.67-1.36)$ \\
\hline 4 & 18.8 & 6652 & 64 & 0.86 & $(0.60-1.23)$ & 0.88 & (0.61-1.26) \\
\hline 5 & 24.6 & 6653 & 64 & 0.87 & $(0.60-1.24)$ & 0.90 & $(0.62-1.29)$ \\
\hline$P_{\text {trend }}$ & & & & 0.39 & & 0.50 & \\
\hline Continuous 1 SD & & & & 0.96 & $(0.86-1.07)$ & 0.98 & $(0.87-1.09)$ \\
\hline \multicolumn{8}{|c|}{ Fat from dairy products ${ }^{5}(\mathrm{~g} /$ day) } \\
\hline $1^{6}$ & 5.3 & 6627 & 70 & 1.00 & & 1.00 & \\
\hline 2 & $11-0$ & 6434 & 62 & 0.90 & $(0.62-1.30)$ & 0.89 & $(0.62-1.29)$ \\
\hline 3 & 15.6 & 6457 & 51 & 0.74 & (0.50-1.08) & 0.72 & $(0.49-1.06)$ \\
\hline 4 & 22.6 & 6325 & 64 & 0.92 & (0.64-1.33) & 0.90 & $(0.62-1.30)$ \\
\hline 5 & 36.2 & 6381 & 93 & 1.33 & $(0.95-1.87)$ & 1.28 & $(0.91-1.80)$ \\
\hline$P_{\text {trend }}$ & & & & 0.11 & & 0.17 & \\
\hline Continuous 1 SD & & & & 1.15 & $(1.03-1.28)$ & 1.13 & $(1.01-1.27)$ \\
\hline
\end{tabular}

Continues on the next page 


\begin{tabular}{|c|c|c|c|c|c|c|c|}
\hline \multirow[b]{2}{*}{ Food item } & \multirow[b]{2}{*}{$\begin{array}{l}\text { Median } \\
\text { intake }^{2}\end{array}$} & \multirow[b]{2}{*}{ PY } & \multicolumn{5}{|c|}{ Total ovarian cancer cases } \\
\hline & & & Cases & $\mathrm{RR}^{3}$ & $(95 \% \mathrm{Cl})$ & $\mathrm{RR}^{4}$ & $(95 \% \mathrm{Cl})$ \\
\hline \multicolumn{8}{|c|}{ Fat from margarines ${ }^{5}$ (g/day) } \\
\hline $1^{6}$ & 9.7 & 6424 & 80 & 1.00 & & 1.00 & \\
\hline 2 & 19.3 & 6368 & 61 & 0.78 & $(0.54-1.12$ & 0.77 & $(0.54-1.11)$ \\
\hline 3 & $26-0$ & 6456 & 69 & 0.87 & $(0.61-1.23)$ & 0.87 & $(0.61-1.23)$ \\
\hline 4 & 32.7 & 6519 & 60 & 0.76 & $(0.53-1.09)$ & 0.75 & $(0.52-1.08)$ \\
\hline 5 & 42.5 & 6357 & 70 & 0.88 & $(0.62-1.25)$ & 0.87 & $(0.61-1.23)$ \\
\hline$P_{\text {trend }}$ & & & & 0.49 & & 0.42 & \\
\hline Continuous 1 SD & & & & 0.94 & $(0.84-1.05)$ & 0.93 & $(0.83-1.05)$ \\
\hline \multicolumn{8}{|c|}{ Saturated fatty acids ${ }^{5,7}$ (g/day) } \\
\hline $1^{6}$ & 23.1 & 6401 & 68 & 1.00 & & 1.00 & \\
\hline 2 & 26.7 & 6427 & 64 & 0.98 & $(0.66-1.46)$ & 0.98 & $(0.66-1.46)$ \\
\hline 3 & 29.2 & 6530 & 57 & 0.88 & $(0.58-1.35)$ & 0.90 & $(0.59-1.37)$ \\
\hline 4 & 32.3 & 6403 & 57 & 0.89 & $(0.57-1.41)$ & 0.89 & $(0.56-1.40)$ \\
\hline 5 & 37.5 & 6363 & 94 & 1.50 & $(0.95-2.38)$ & 1.48 & $(0.94-2.34)$ \\
\hline$P_{\text {trend }}$ & & & & 0.12 & & 0.14 & \\
\hline Continuous 1 SD & & & & 1.23 & $(1.05-1.43)$ & 1.21 & $(1.04-1.41)$ \\
\hline \multicolumn{8}{|c|}{ Monounsaturated fatty acids ${ }^{5,7}$ (g/day) } \\
\hline $1^{6}$ & 21.7 & 6410 & 66 & 1.00 & & 1.00 & \\
\hline 2 & 25.1 & 6418 & 78 & 1.12 & $(0.78-1.64)$ & 1.16 & $(0.80-1.68)$ \\
\hline 3 & 27.4 & 6381 & 65 & 0.95 & $(0.63-1.42)$ & 1.00 & $(0.66-1.51)$ \\
\hline 4 & 29.8 & 6436 & 66 & 0.92 & $(0.60-1.41)$ & 0.97 & $(0.63-1.49)$ \\
\hline 5 & 33.5 & 6479 & 65 & 0.84 & $(0.52-1.37)$ & 0.90 & $(0.55-1.46)$ \\
\hline$P_{\text {trend }}$ & & & & 0.29 & & 0.44 & \\
\hline Continuous 1 SD & & & & 0.92 & $(0.78-1.09)$ & 0.85 & $(0.80-1.12)$ \\
\hline \multicolumn{8}{|c|}{ Polyunsaturated fatty acids ${ }^{5,7}$ (g/day) } \\
\hline $1^{6}$ & $8-0$ & 6381 & 86 & 1.00 & & 1.00 & \\
\hline 2 & 11.4 & 6412 & 54 & 0.63 & $(0.44-0.91)$ & 0.65 & $(0.44-0.94)$ \\
\hline 3 & 14.1 & 6473 & 62 & 0.73 & $(0.50-1.05)$ & 0.78 & $(0.54-1.14)$ \\
\hline 4 & 17.6 & 6411 & 78 & 0.91 & $(0.64-1.29)$ & 0.93 & $(0.66-1.32)$ \\
\hline 5 & 23.2 & 6446 & 60 & 0.68 & $(0.47-0.99)$ & 0.89 & $(0.47-1.01)$ \\
\hline$P_{\text {trend }}$ & & & & 0.30 & & 0.33 & \\
\hline Continuous 1 SD & & & & 0.90 & $(0.79-1.03)$ & 0.90 & $(0.79-1.03)$ \\
\hline \multicolumn{8}{|c|}{ trans Unsaturated fatty acids ${ }^{5,7}$ (g/day) } \\
\hline $1^{6}$ & 1.5 & 6435 & 63 & 1.00 & & 1.00 & \\
\hline 2 & 2.1 & 6446 & 55 & 0.92 & $(0.62-1.35)$ & 0.91 & $(0.62-1.35)$ \\
\hline 3 & 2.4 & 6407 & 59 & 0.99 & $(0.67-1.47)$ & 0.99 & $(0.67-1.47)$ \\
\hline 4 & 2.8 & 6420 & 77 & 1.31 & $(0.90-1.91)$ & 1.33 & (0.91-1.94) \\
\hline 5 & 3.5 & 5417 & 86 & 1.49 & $(1.03-2.16)$ & 1.51 & $(1.04-2.20)$ \\
\hline$P_{\text {trend }}$ & & & & 0.01 & & 0.01 & \\
\hline Continuous 1 SD & & & & 1.14 & $(1.02 / 1.26)$ & 1.14 & $(1.03-1.28)$ \\
\hline
\end{tabular}

PY (person years at risk)

${ }^{1}$ Relative risks were derived from Cox regression analyses

${ }^{2}$ Median intake in subcohort.

${ }^{3}$ Adjusted for age (y) and total energy intake (kcal)

${ }^{4}$ Adjusted for age (y)-total energy intake (kcal)-parity (number of children) and use of oral contraceptives (ever versus never)

${ }^{5}$ Energy adjusted intake

${ }^{6}$ Reference category

${ }^{7}$ Additionally adjusted for total energy-adjusted fat intake 
Table 3: Relative risks (RR) and 95\% confidence intervals (CI) for ovarian cancer according to quintiles and categories of intake of fresh meat-types of fresh meat-processed meat - NLCS 1986-2002 ${ }^{1}$

\begin{tabular}{|c|c|c|c|c|c|c|c|}
\hline \multirow[b]{2}{*}{ Food item } & \multirow[b]{2}{*}{$\begin{array}{l}\text { Median } \\
\text { intake }^{2}\end{array}$} & \multirow[b]{2}{*}{ PY } & \multicolumn{5}{|c|}{ Total ovarian cancer cases } \\
\hline & & & Cases & $\mathrm{RR}^{3}$ & $(95 \% \mathrm{Cl})$ & $\mathrm{RR}^{4}$ & $(95 \% \mathrm{Cl})$ \\
\hline \multicolumn{8}{|c|}{ Total fresh meat (g/day) ${ }^{5}$} \\
\hline $1^{6}$ & 45.2 & 6300 & 52 & 1.00 & & 1.00 & \\
\hline 2 & 74.4 & 6500 & 77 & 1.44 & $(0.99-2.09)$ & 1.45 & $(1.00-2.13)$ \\
\hline 3 & $92-0$ & 6367 & 80 & 1.51 & $(1.04-2.20)$ & 1.54 & $(1.06-2.42)$ \\
\hline 4 & 107.5 & 6519 & 80 & 1.48 & $(1.02-2.15)$ & 1.49 & $(1.03-2.17)$ \\
\hline 5 & 145.8 & 6438 & 51 & 0.94 & $(0.62-1.42)$ & 0.97 & $(0.63-1.47)$ \\
\hline \multicolumn{4}{|c|}{$P_{\text {trend }}$} & 0.86 & & 0.96 & \\
\hline \multicolumn{4}{|c|}{ Continuous ( $25 \mathrm{~g} /$ day intake increment) } & 0.97 & $(0.91-1.04)$ & 0.98 & $(0.91-1.04)$ \\
\hline \multicolumn{8}{|c|}{ Fresh red meat $(\mathrm{g} / \text { day })^{7}$} \\
\hline $1^{6}$ & 36.2 & 6491 & 51 & 1.00 & & 1.00 & \\
\hline 2 & 61.3 & 6314 & 77 & 1.54 & $(1.06-2.25)$ & 1.58 & $(1.08-2.30)$ \\
\hline 3 & 77.9 & 6389 & 73 & 1.45 & $(0.99-2.12)$ & 1.47 & $(1.00-2.16)$ \\
\hline 4 & 95.6 & 6477 & 92 & 1.77 & $(1.23-2.56)$ & 1.78 & $(1.23-2.58)$ \\
\hline 5 & 129.6 & 6453 & 47 & 0.91 & $(0.60-1.38)$ & 0.93 & $(0.61-1.42)$ \\
\hline \multicolumn{4}{|l|}{$P_{\text {trend }}$} & 0.90 & & 0.85 & \\
\hline Continuo & ake increr & & & 0.98 & $(0.92-1.05)$ & 0.98 & $(0.92-1.05)$ \\
\hline \multicolumn{8}{|c|}{ Beef (g/day) } \\
\hline $1^{6}$ & 2.2 & 6445 & 68 & 1.00 & & 1.00 & \\
\hline 2 & 10.7 & 6471 & 53 & 0.77 & $(0.53-1.13)$ & 0.77 & $(0.52-1.13)$ \\
\hline 3 & 18.9 & 6478 & 58 & 0.98 & $(0.68-1.41)$ & 0.98 & $(0.68-1.42)$ \\
\hline 4 & 30.7 & 6391 & 67 & 0.97 & $(0.67-1.40)$ & 0.95 & $(0.66-1.38)$ \\
\hline 5 & 50.4 & 6339 & 84 & 1.21 & $(0.86-1.72)$ & 1.15 & $(0.81-1.64)$ \\
\hline \multicolumn{4}{|l|}{$P_{\text {trend }}$} & 0.14 & & 0.23 & \\
\hline \multicolumn{4}{|c|}{ Continuous ( $25 \mathrm{~g} /$ day intake increment) } & 1.09 & $(0.97-1.22)$ & 1.07 & $(0.95-1.20)$ \\
\hline \multicolumn{8}{|c|}{ Pork (g/day) } \\
\hline $1^{6}$ & 3.5 & 6489 & 62 & 1.00 & & 1.00 & \\
\hline 2 & 18.3 & 6335 & 74 & 1.23 & $(0.85-1.76)$ & 1.26 & $(0.87-1.81)$ \\
\hline 3 & 31.1 & 6417 & 71 & 1.18 & $(0.81-1.69)$ & 1.21 & $(0.84-1.76)$ \\
\hline 4 & 44.7 & 6410 & 67 & 1.09 & $(0.75-1.58)$ & 1.14 & $(0.78-1.66)$ \\
\hline 5 & 71.2 & 6472 & 66 & 1.07 & $(0.73-1.55)$ & 1.08 & $(0.75-1.59)$ \\
\hline \multicolumn{3}{|l|}{$P_{\text {trend }}$} & & 0.99 & & 0.88 & \\
\hline \multicolumn{4}{|c|}{ Continuous (25g/day intake increment) } & 0.95 & $(0.86-1.04)$ & 0.96 & $(0.87-1.05)$ \\
\hline \multicolumn{8}{|c|}{ Minced meat $(\mathrm{g} / \text { day })^{8}$} \\
\hline $1^{6}$ & 0 & 6357 & 66 & 1.00 & & 1.00 & \\
\hline 2 & 7.6 & 6281 & 67 & 1.02 & $(0.71-1.47)$ & 1.06 & $(0.73-1.53)$ \\
\hline 3 & 13.4 & 6722 & 83 & 1.19 & $(0.84-1.68)$ & 1.26 & $(0.89-1.80)$ \\
\hline 4 & 21.4 & 6366 & 69 & 1.05 & $(0.73-1.52)$ & 1.13 & $(0.78-1.63)$ \\
\hline 5 & 36.6 & 6398 & 55 & 0.82 & $(0.56-1.21)$ & 0.86 & $(0.59-1.27)$ \\
\hline \multicolumn{4}{|l|}{$P_{\text {trend }}$} & 0.43 & & 0.64 & \\
\hline Continuo & ake increr & & & 0.89 & $(0.74-1.07)$ & 0.91 & $(0.76-1.09)$ \\
\hline \multicolumn{8}{|c|}{ Liver (g/day) ${ }^{9}$} \\
\hline $1^{5}$ & 0 & 21519 & 227 & 1.00 & & 1.00 & \\
\hline 2 & 3.3 & 10604 & 113 & 1.04 & $(0.81-1.33)$ & 1.07 & $(0.84-1.38)$ \\
\hline \multicolumn{4}{|c|}{ Continuous (25g/day intake increment) } & 1.15 & $(0.59-2.25)$ & 1.29 & $(0.66-2.52)$ \\
\hline Chicken (g/ & & & & & & & \\
\hline $1^{5}$ & 0 & 7530 & 76 & 1.00 & & 1.00 & \\
\hline 2 & 5.3 & 8304 & 95 & 1.15 & $(0.83-1.59)$ & 1.18 & $(0.85-1.64)$ \\
\hline 3 & 13.2 & 7436 & 79 & 1.07 & $(0.76-1.51)$ & 1.14 & $(0.81-1.61)$ \\
\hline 4 & 22.8 & 8853 & 90 & 1.01 & $(0.73-1.41)$ & 1.06 & $(0.76-1.48)$ \\
\hline $\begin{array}{l}P_{\text {trend }} \\
\text { Continuol }\end{array}$ & & & & 0.93 & & 0.83 & \\
\hline increment) & & & & 0.94 & $(0.78-1.13)$ & 0.96 & $(0.80-1.14)$ \\
\hline
\end{tabular}




\begin{tabular}{|c|c|c|c|c|c|c|c|}
\hline \multirow[b]{2}{*}{ Food item } & \multirow[b]{2}{*}{$\begin{array}{l}\text { Median } \\
\text { intake }^{2}\end{array}$} & \multirow[b]{2}{*}{ PY } & \multicolumn{5}{|c|}{ Total ovarian cancer cases } \\
\hline & & & Cases & $\mathrm{RR}^{3}$ & $(95 \% \mathrm{Cl})$ & $\mathrm{RR}^{4}$ & $(95 \% \mathrm{Cl})$ \\
\hline \multicolumn{8}{|c|}{ Processed meat (g/day) } \\
\hline $1^{6}$ & 0 & 6725 & 80 & 1.00 & & 1.00 & \\
\hline 2 & 2.7 & 6268 & 53 & 0.71 & $(0.49-1.03)$ & 0.71 & $(0.49-1.03)$ \\
\hline 3 & 6.8 & 6436 & 70 & 0.91 & $(0.65-1.29)$ & 0.91 & $(0.64-1.29)$ \\
\hline 4 & 13.0 & 9227 & 70 & 0.93 & $(0.66-1.32)$ & 0.93 & $(0.65-1.31)$ \\
\hline 5 & 25.6 & 6466 & 67 & 0.85 & $(0.60-1.21)$ & 0.83 & $(0.59-1.20)$ \\
\hline \multicolumn{4}{|c|}{$P_{\text {trend }}$} & 0.80 & & 0.74 & \\
\hline \multicolumn{4}{|c|}{ Continuous ( $25 \mathrm{~g} /$ day intake increment) } & 0.97 & $(0.75-1.25)$ & 0.96 & $(0.75-1.23)$ \\
\hline \multicolumn{8}{|c|}{ Fish (g/day) ${ }^{9}$} \\
\hline $1^{5}$ & 0 & 9879 & 102 & 1.00 & & 1.00 & \\
\hline 2 & 4.6 & 7057 & 82 & 1.14 & $(0.83-1.57)$ & 1.15 & $(0.84-1.58)$ \\
\hline 3 & 15.5 & 9729 & 101 & 1.00 & $(0.75-1.36)$ & 1.02 & $(0.76-1.38)$ \\
\hline 4 & 28.2 & 5458 & 55 & 0.98 & $(0.69-1.39)$ & 1.01 & $(0.71-1.43)$ \\
\hline \multicolumn{4}{|l|}{$P_{\text {trend }}$} & 0.81 & & 0.94 & \\
\hline \multicolumn{4}{|c|}{ Continuous ( $25 \mathrm{~g} /$ day intake increment) } & 0.90 & $(0.73-1.10)$ & 0.91 & $(0.74-1.12)$ \\
\hline \multicolumn{8}{|c|}{ PY (person years at risk). } \\
\hline \multicolumn{8}{|c|}{$\begin{array}{l}\text { PY (person years at risk). } \\
{ }^{1} \text { Relative risks were derived from Cox regression analyses. }\end{array}$} \\
\hline \multicolumn{8}{|c|}{$\begin{array}{l}{ }^{2} \text { Median intake in subcohort. }\end{array}$} \\
\hline \multicolumn{8}{|c|}{${ }^{3}$ Adjusted for age (yrs) and total energy intake (kcal). } \\
\hline \multirow{2}{*}{\multicolumn{8}{|c|}{$\begin{array}{l}{ }^{4} \text { Adjusted for age (yrs), total energy intake (kcal), parity (number of children) and use of oral contraceptives (ever } \\
\text { versus never). }\end{array}$}} \\
\hline & & & & & & & \\
\hline \multicolumn{8}{|c|}{$\begin{array}{l}{ }^{5} \text { Including all types of meat (except processed meat) and chicken. } \\
{ }^{6} \text { Reference category. }\end{array}$} \\
\hline \multicolumn{8}{|c|}{${ }^{7}$ Including beef-pork-minced meat-liver and other meat. } \\
\hline \multicolumn{8}{|c|}{${ }^{8}$ Including beef and pork. } \\
\hline \multicolumn{8}{|c|}{${ }^{9}$ Categorical cutpoints are for liver: 0->0 g/day; chicken: 0, 0-13.2, $13.2-22.8, \geq 22.8 \mathrm{~g} /$ day; fish: 0, 0-10, 10-20, $\geq 20$} \\
\hline
\end{tabular}

For trans-unsaturated fatty acids, a statistically significant increased risk of ovarian cancer for the highest versus the lowest quintile of TFA intake (multivariable RR $=1.51,95 \% \mathrm{Cl}$ : $1.04,2.20$ ) was found. Also, a dose-response relation was observed ( $p$ for trend $=0.01$ ) and a statistically significant increased ovarian cancer risk per 1 SD intake of TFA intake as a continuous variable was observed (multivariable $\mathrm{RR}=1.14,95 \% \mathrm{Cl}: 1.03,2.28$ ) (Table 2). When including all fatty acids together in one model, the association between SF, MUFA, PUFA and TFA and ovarian cancer risk attenuated slightly but the direction of the association did not change appreciably (results not shown). A significant inverse trend across all quintiles of MUFA intake and ovarian cancer was observed $(P$-trend $=0.03$ ) but the RR for 1 SD increase was similar (RR $0.84 ; 95 \% \mathrm{Cl} 0.88,1.14$ ) (results not shown).

Intake of total fresh meat and fresh red meat did not show a clear monotonic increasing relation with ovarian cancer risk, but statistically significantly increased RRs in women in the intermediate quintiles of intake i.e. quintiles 2 through 4 (multivariable RRs for total fresh meat $1.00,1.45,1.54,1.48$ and 0.97 , respectively; multivariable RRs for fresh red meat 1.00, 1.58, 1.47, 1.78 and 0.93 respectively) (Table 3). After adjustment for age and energy, no association was found between the intake of beef, pork, minced meat, liver, chicken, processed meat and fish separately and the risk of ovarian cancer (Table 3). These findings remained after the inclusion of the confounding variables in the multivariable model. Additional adjustment for the complementary meat items in the multivariable models did not change the results notably (results not shown). For beef and pork 
consumption the proportional hazards assumption was violated. We observed a statistically significant interaction with time for beef $(P$-interaction $=0.022)$ but not for pork $(P$ interaction $=0.092$ ). When stratifying the follow-up period into three categories (data not shown), only during the first 5 years of follow-up a statistical significantly increased risk of ovarian cancer was observed in quintiles 3 to 5 compared with the lowest quintile of beef intake (RRs 1.00, 0.99, 2.30, 2.04, 3.02, respectively) indicating that the association between beef intake and ovarian cancer risk attenuates over time.

When we tested for interactions by use of oral contraceptives or parity, we observed no statistically significant interaction (results not shown). Stratified analyses (see Supplemental Table 1) showed statistically significant positive associations with ovarian cancer only in parous women and those never using oral contraceptives as regards to animal fat, dairy fat, TFA, SFA intake whereas an inverse association with PUFA was observed in parous women only. Stratified analyses regarding meat consumption yielded similar results compared to the overall assessment (results not shown). Due to the limited use of post-menopausal hormone replacement therapy in our cohort, we were not able to test this interaction.

\section{DISCUSSION}

In this large prospective cohort study, a comprehensive set of different sources and subtypes of fat and meat was examined in relation to ovarian cancer risk. Results showed a statistically significant positive association between dietary intake of trans unsaturated fatty acids and the risk of epithelial ovarian cancer.

Previously, a meta-analysis of predominantly case control studies [42] has shown an increased risk of ovarian cancer for the highest compared to the lowest intakes of total fat, animal fat and saturated fat (RRs 1.24, 1.20 and 1.70, respectively). Nevertheless, incidence of ovarian cancer was not associated with total fat or any subtype of dietary fat intake according the lowa Women's Health Study [20] and the Nurses' Health Study [19], whereas case control studies yielded mixed results [8, 13-17]. Although comparable to previous studies, the range of energy intake from total fat in the present study was small (varying from $32 \%$ in the lowest to $46 \%$ in the fifth quintile) which might have contributed to the lack of association observed. Moreover, habitual fat intake below 30 energy\% is considered to be difficult to achieve in Western populations. Yet, recent results from the Women's Health Initiative Dietary Modification Trial suggested that a low-fat dietary pattern (24.3 vs. 35.1 energy\% from fat after one year from baseline for the intervention and control group, respectively) may reduce the incidence of ovarian cancer among healthy postmenopausal women (RR 0.60, low fat diet compared to regular diet after four to eight y of follow-up) [43].

Thus far, epidemiological evidence linking TFA to any type of cancer is limited [44]; both the Nurses' Health Study [19] and a pooled analysis [45] including data of 4 cohort studies found no evidence of an association between intake of TFA and ovarian cancer risk. In contrast, we found a positive association between dietary intake of TFA and the risk of ovarian cancer. After the substantial reduction of TFAs in margarines in Europe in 1995 [46], the remaining TFAs in the diet are mainly consumed from fast food products, cakes 
and biscuits [47]. Although these specific dietary sources are likely to be closely related to other lifestyle factors or food specific chemicals such as acrylamide, adjustment for these did not attenuate the association between TFAs and ovarian cancer risk substantially (results not shown). Although a considerable latency period may exist between fat intake and cancer risk, we did not observe a statistically significant interaction with time of followup $(P$-interaction $=0.46)$ suggesting that the strength of the association did not follow the decline in TFA exposure.

Consumption of fat from plant foods was not associated with ovarian cancer risk in two case control studies $[8,12]$ or in two prospective cohort studies in the US $[19,20]$ and a pooled analysis of 9 cohort studies [45]. Conversely, the present results suggest an inverse association between plant-based fat consumption and ovarian cancer risk above the threshold of $4.5 \mathrm{~g} / \mathrm{d}$. Yet, the direction of the association depends on the choice of the reference category; changing this to a higher category would show an increased risk for those with the lowest intake. Nevertheless, previous studies often lack to specify whether hydrogenated vegetable oils, characteristically more comparable to animal fat, are included in this fat source, making comparisons between studies difficult. In the current analyses, plant based fat accounts, on average, for only $12 \%$ of total fat intake. However, the intake is greatly dispersed and likely to reflect a dietary pattern in the extremes. Regarding this, factor analyses previously identified five stable dietary patterns among women in the NLCS [48]. Although consumption of fat from plant foods was inversely correlated with the "fat dairy pattern" ( $r=0.28, P<0.001)$ (high loadings on potatoes, non-fermented whole milk, margarine, and sweet sandwich spread) adjustment for this pattern did not change the results noticeably (results not shown). Moreover, a borderline statistically significant trend towards a protective effect of ovarian cancer for higher intakes of plant based fat relative to animal fat $(P$-trend $=0.086)$ was observed in the present study.

We observed a higher ovarian cancer risk per 1 SD increase in dairy fat intake but because there was no increasing trend through all quintiles we are prudent interpreting these findings. More studies are warranted to further examine this association particularly since longitudinal epidemiological evidence is limited and conflicting $[18,19]$.

To date, a direct association between meat consumption and ovarian cancer risk has been proposed by several [5-9], but not all case control studies [10-13, 19, 20, 22]. Two prospective cohort studies that included a large proportion of vegetarians or low meat consumers were indicative of a positive association between total meat consumption and ovarian cancer risk $[18,49]$. In contrast, four other cohort studies [19-22] and a recent meta-analysis [50] with smaller numbers of low or no meat consumers found no clear evidence for an association with total or red meat consumption. Yet, our results suggest that women at the extremes of the total fresh meat and fresh red meat consumption spectrum may be at a lower risk of developing ovarian cancer compared to those with intermediate consumption. Such an association has not been reported previously and may reflect on a specific dietary and lifestyle pattern high in fresh meat consumption. Nevertheless, negligible attenuation in the association with ovarian cancer was observed after adjustment for the dietary pattern that correlated most strongly with total fresh meat consumption ('pork, processed meat and potatoes pattern') [48] $(r=0.29, P<0.001)$ or when adjusting for other potential covariates. Although our reference category is characterized 
by a considerable wide range of total fresh meat consumption (0-62.5 g/day; 0-7 d/week), changing this to a smaller, and more extreme category did not change the results appreciably.

In accordance with the one study previously examining effect modification by hormonal and reproductive factors on the association between dietary fat and meat intake and ovarian cancer, we observed null results (possibly due to small numbers) [45]. Yet, the effect sizes regarding fat exposures were generally more pronounced in never pill users and parous women suggesting that hormonal pathways may be of significance in the etiology of ovarian cancer. However; in our cohort relatively few women were nulliparous and those reporting oral contraceptive use were significantly younger than those not.

Our analyses have been performed using baseline FFQ data only resulting in an inability to assess and account for changes in intake and food compositions over time (e.g. TFA content). However, the validity of the FFQ has been tested and shown to be representative for dietary habits over a period of at least 5 years [35]. The prospective design reduced the potential for recall bias, and the nearly complete follow-up of cases and subcohort members, makes selection bias unlikely. Detailed information on diet and potential risk factors of ovarian cancer enabled us to control for most known ovarian cancer risk factors, although misclassification of exposure may have occurred. Regression parameters proved to be robust estimators since adjustment for potential covariates did not change them appreciable.

In conclusion, this prospective study suggests that trans unsaturated fatty acids, but no other types of fat or meat, may be associated with increased ovarian cancer risk in this population of postmenopausal women. The risk attributable to different sources and types of fats and interactions by oral contraceptive use and parity warrant further investigation.

\section{REFERENCES}

1. Riman, T., S. Nilsson, and I.R. Persson, Review of epidemiological evidence for reproductive and hormonal factors in relation to the risk of epithelial ovarian malignancies. Acta Obstet Gynecol Scand, 2004. 83(9): p. 783-95.

2. Beral, V., et al., Ovarian cancer and oral contraceptives: collaborative reanalysis of data from 45 epidemiological studies including 23,257 women with ovarian cancer and 87,303 controls. Lancet, 2008. 371(9609): p. 303-14.

3. Rose, D.P., A.P. Boyar, and E.L. Wynder, International comparisons of mortality rates for cancer of the breast, ovary, prostate, and colon, and per capita food consumption. Cancer, 1986. 58(11): p. 2363-71.

4. Dunn, J.E., Cancer epidemiology in populations of the United States-with emphasis on Hawaii and California-and Japan. Cancer Res, 1975. 35(11 Pt. 2): p. 3240-5.

5. Bosetti, C., et al., Diet and ovarian cancer risk: a case-control study in Italy. Int J Cancer, 2001. 93(6): p. 911-5.

6. La Vecchia, C., et al., Dietary factors and the risk of epithelial ovarian cancer. J Natl Cancer Inst, 1987. 79(4): p. 663-9.

7. Mori, M., et al., Reproductive, genetic, and dietary risk factors for ovarian cancer. Am J Epidemiol, 1988. 128(4): p. 771-7.

8. Shu, X.O., et al., Dietary factors and epithelial ovarian cancer. Br J Cancer, 1989. 59(1): p. 92-6.

9. Tavani, A., et al., Red meat intake and cancer risk: a study in Italy. Int J Cancer, 2000. 86(3): p. 425-8.

10. Engle, A., J.E. Muscat, and R.E. Harris, Nutritional risk factors and ovarian cancer. Nutr Cancer, 1991. 15(3-4): p. 239-47. 
11. Hu, J., et al., Meat and fish consumption and cancer in Canada. Nutr Cancer, 2008. 60(3): p. 313-24.

12. Salazar-Martinez, E., et al., Nutritional determinants of epithelial ovarian cancer risk: a case-control study in Mexico. Oncology, 2002. 63(2): p. 151-7.

13. Zhang, M., et al., Diet and ovarian cancer risk: a case-control study in China. Br J Cancer, 2002. 86(5): p. 712-7.

14. Risch, H.A., et al., Dietary fat intake and risk of epithelial ovarian cancer. J Natl Cancer Inst, 1994. 86(18): p. 1409-15.

15. Cramer, D.W., et al., Dietary animal fat in relation to ovarian cancer risk. Obstet Gynecol, 1984. 63(6): p. 833-8.

16. Byers, T., et al., A case-control study of dietary and nondietary factors in ovarian cancer. J Natl Cancer Inst, 1983. 71(4): p. 681-6.

17. Slattery, M.L., et al., Nutrient intake and ovarian cancer. Am J Epidemiol, 1989. 130(3): p. 497-502.

18. Kiani, F., et al., Dietary risk factors for ovarian cancer: the Adventist Health Study (United States). Cancer Causes Control, 2006. 17(2): p. 137-46.

19. Bertone, E.R., et al., Dietary fat intake and ovarian cancer in a cohort of US women. Am J Epidemiol, 2002. 156(1): p. 22-31.

20. Kushi, L.H., et al., Prospective study of diet and ovarian cancer. Am J Epidemiol, 1999. 149(1): p. 21-31.

21. Schulz, M., et al., No association of consumption of animal foods with risk of ovarian cancer. Cancer Epidemiol Biomarkers Prev, 2007. 16(4): p. 852-5.

22. Larsson, S.C. and A. Wolk, No association of meat, fish, and egg consumption with ovarian cancer risk. Cancer Epidemiol Biomarkers Prev, 2005. 14(4): p. 1024-5.

23. World Cancer Research Fund/American Institute for Cancer Research, Food, Nutrition, Physical Activity, and the Prevention of Cancer: a Global Perspective. 2007, AICR: Washington DC.

24. Lukanova, A. and R. Kaaks, Endogenous hormones and ovarian cancer: epidemiology and current hypotheses. Cancer Epidemiol Biomarkers Prev, 2005. 14(1): p. 98-107.

25. Risch, H.A., Hormonal etiology of epithelial ovarian cancer, with a hypothesis concerning the role of androgens and progesterone. J Natl Cancer Inst, 1998. 90(23): p. 1774-86.

26. Cramer, D.W. and W.R. Welch, Determinants of ovarian cancer risk. II. Inferences regarding pathogenesis. J Natl Cancer Inst, 1983. 71(4): p. 717-21.

27. Armstrong, B.K., et al., Diet and reproductive hormones: a study of vegetarian and nonvegetarian postmenopausal women. J Natl Cancer Inst, 1981. 67(4): p. 761-7.

28. Holmes, M.D., et al., Dietary fat intake and endogenous sex steroid hormone levels in postmenopausal women. J Clin Oncol, 2000. 18(21): p. 3668-76.

29. Wu, A.H., et al., Soy intake and other lifestyle determinants of serum estrogen levels among postmenopausal Chinese women in Singapore. Cancer Epidemiol Biomarkers Prev, 2002. 11(9): p. 84451.

30. Wu, A.H., M.C. Pike, and D.O. Stram, Meta-analysis: dietary fat intake, serum estrogen levels, and the risk of breast cancer. J Natl Cancer Inst, 1999. 91(6): p. 529-34.

31. Goldin, B.R., et al., Estrogen excretion patterns and plasma levels in vegetarian and omnivorous women. N Engl J Med, 1982. 307(25): p. 1542-7.

32. Ferguson, L.R., Natural and human-made mutagens and carcinogens in the human diet. Toxicology, 2002. 181-182: p. 79-82.

33. van den Brandt, P.A., et al., A large-scale prospective cohort study on diet and cancer in The Netherlands. J Clin Epidemiol, 1990. 43(3): p. 285-95.

34. Goldbohm, R.A., P.A. van den Brandt, and E. Dorant, Estimation of the coverage of Dutch municipalities by cancer registries and PALGA based on hospital discharge data. Tijdschrift Soc Gezondheidsz, 1994. 72: p. 80-84.

35. Goldbohm, R.A., et al., Validation of a dietary questionnaire used in a large-scale prospective cohort study on diet and cancer. Eur J Clin Nutr, 1994. 48(4): p. 253-65.

36. Nevo table: Dutch Food Composition Table 1986-1987 (in Dutch). 1986, The Hague: Voorlichtingsbureau voor de voeding.

37. van Poppel, G., Intake of trans fatty acids in western Europe: the TRANSFAIR study. Lancet, 1998. 351(9109): p. 1099.

38. Goldbohm, R.A., et al., Reproducibility of a food frequency questionnaire and stability of dietary habits determined from five annually repeated measurements. Eur J Clin Nutr, 1995. 49(6): p. 420-9. 
39. Willett, W.C., Implications of total energy intake for epidemiologic studies of breast and large-bowel cancer. Am J Clin Nutr, 1987. 45(1 Suppl): p. 354-60.

40. Volovics, A. and P.A. van den Brandt, Methods for the analysis of case-cohort studies. Biom J, 1997(39): p. 195-214.

41. Lin, D.Y. and L.J. Wei, The robust inference for the Cox proportional hazards model. J AM Stat Assoc, 1989(84): p. 1074-8.

42. Huncharek, M. and B. Kupelnick, Dietary fat intake and risk of epithelial ovarian cancer: a metaanalysis of 6,689 subjects from 8 observational studies. Nutr Cancer, 2001. 40(2): p. 87-91.

43. Prentice, R.L., et al., Low-fat dietary pattern and cancer incidence in the Women's Health Initiative Dietary Modification Randomized Controlled Trial. J Natl Cancer Inst, 2007. 99(20): p. 1534-43.

44. Thompson, A.K., et al., Trans-fatty acids and cancer: the evidence reviewed. Nutr Res Rev, 2008. 21(2): p. $174-88$.

45. Genkinger, J.M., et al., A pooled analysis of 12 cohort studies of dietary fat, cholesterol and egg intake and ovarian cancer. Cancer Causes Control, 2006. 17(3): p. 273-85.

46. Meijer, G.W. and J.A. Weststrate, Interesterification of fats in margarine: effect on blood lipids, blood enzymes, and hemostasis parameters. Eur J Clin Nutr, 1997. 51(8): p. 527-34.

47. Katan, M.B., [Elimination of all trans fatty acids]. Ned Tijdschr Geneeskd, 2008. 152(6): p. 302-7.

48. Balder, H.F., et al., Common and country-specific dietary patterns in four European cohort studies. J Nutr, 2003. 133(12): p. 4246-51.

49. Key, T.J., et al., Cancer incidence in British vegetarians. Br J Cancer, 2009. 101(1): p. 192-7.

50. Kolahdooz, F., et al., Meat, fish, and ovarian cancer risk: Results from 2 Australian case-control studies, a systematic review, and meta-analysis. Am J Clin Nutr, 2010. 91(6): p. 1752-63. 


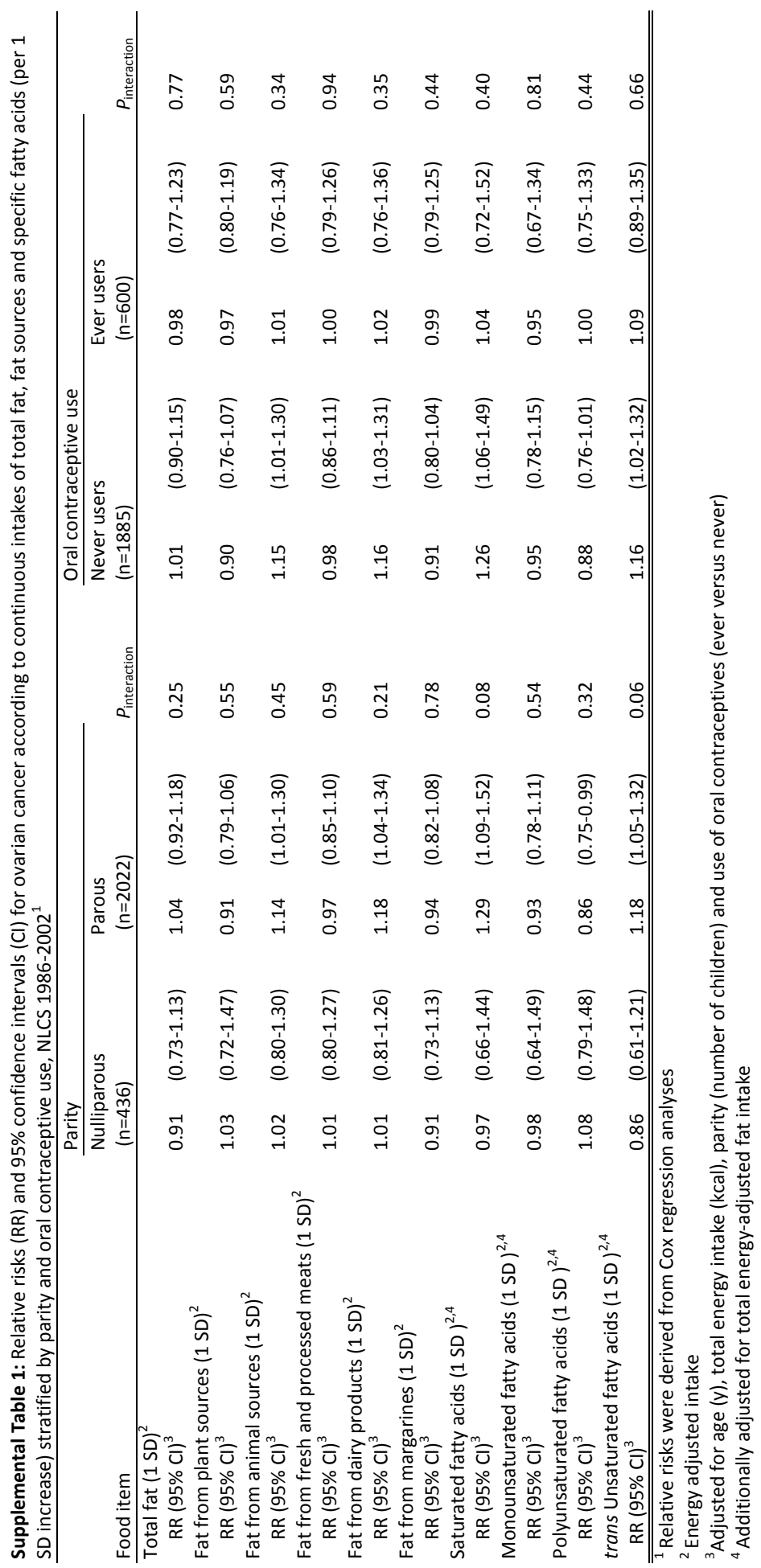





\title{
CHAPTER 8
}

\section{Meat-related mutagen exposure, xenobiotic metabolizing gene polymorphisms and the risk of advanced colorectal adenoma and cancer}

\author{
Anne MJ Gilsing \\ Sonja I Berndt \\ Elizabeth H Ruder \\ Barry I Graubard \\ Leah M Ferrucci \\ Laura Burdett \\ Joel L Weissfeld \\ Amanda J Cross \\ and Rashmi Sinha
}

Carcinogenesis 2013;34(12):2757-66 


\section{ABSTRACT}

Background: Meat mutagens, including heterocyclic amines (HCAs), polycyclic aromatic hydrocarbons (PAHs), and $\mathrm{N}$-nitroso compounds (NOCs), may be involved in colorectal carcinogenesis depending on their activation or detoxification by phase I and II xenobiotic metabolizing enzymes (XME).

Methods: Using unconditional logistic regression to estimate odds ratios (OR) and $95 \%$ confidence intervals $(\mathrm{Cl})$, we examined the intake of five meat mutagens and $>300$ single nucleotide polymorphisms (SNPs) in $18 \mathrm{XME}$ genes in relation to advanced colorectal adenoma (1,205 cases; 1,387 controls), and colorectal cancer (370 cases; 401 controls) within the Prostate, Lung, Colorectal, and Ovarian Cancer Screening Trial. Dietary intake of meat mutagens was assessed using a food frequency questionnaire (FFQ) with a detailed meat-cooking module.

Results: An interaction was observed between 2-amino-3,8-dimethylimidazo[4,5f]quinoxaline (MelQx) intake and the NAT1 polymorphism rs6586714 in the adenoma study $(P$-interaction $=0.001)$. Among individuals carrying a GG genotype, high MelQx intake was associated with a $43 \%$ increased risk of adenoma $(95 \% \mathrm{Cl}, 1.11-1.85 ; P$-trend $=0.07$ ), whereas the reverse was observed among carriers of the $A$ variant $(\mathrm{OR}=0.50 ; 95 \% \mathrm{Cl}, 0.30$ $0.84 ; P$-trend $=0.01)$. In addition, we observed some suggestive $(P<0.05)$ modifying effects for SNPs in other XME genes (UGT1A, CYP2E1, EPHX1, AHR, and GSTM3), but these were not significant after adjustment for multiple testing.

Conclusion: This large and comprehensive study of XME genes, meat mutagens and the risk of colorectal tumours found that a NAT1 polymorphism modified the association between MeIQx intake and colorectal adenoma risk. 


\section{INTRODUCTION}

Experimental evidence suggests that the carcinogenic potential of several meat specific mutagens may be one of the underlying causal factors for the well-established epidemiological association between red and processed meat consumption and colorectal cancer risk [1]. Heterocyclic amines (HCAs) are formed when meat is cooked well-done at high temperature $[2,3]$. Grilled meat may also contain polycyclic aromatic hydrocarbons (PAHs) from smoke that coats the meat. PAHs are also present in cigarette smoke, the environment, and in other food; although concentrations in foods not prepared by grilling or smoking are minor by comparison [2,3]. $\mathrm{N}$-nitroso compounds (NOCs) are formed from nitrate and nitrite that are added as preservatives to processed meat [4]. Despite the strong carcinogenic potential of HCAs, PAHs, and NOCs observed in animal studies [5-9], evidence in humans remains inconsistent [1].

HCAs, PAHs, and NOCs undergo a series of chemical reactions in the human body during which they can be activated or detoxified by phase I and phase II xenobiotic metabolizing enzymes (XMEs) [10, 11]. Single nucleotide polymorphisms (SNPs) in the genes encoding these XMEs may modify the ability to activate or detoxify carcinogens. Previous studies examining interactions between XME polymorphisms, meat consumption and the risk of colorectal adenomas or carcinomas have reported mixed results [12-26]. In addition, most previous studies had a small number of cases or examined only a small set of SNPs from a limited number of candidate genes. Because the balance of activating and detoxifying enzymes is thought to influence carcinogen metabolism [27], comprehensive studies including numerous markers across multiple genes involved in xenobiotic metabolism are essential for studying this complex association. Furthermore, the inconsistencies in the data may partly result from the inability of most studies to estimate specific HCAs, PAHs, and nitrite/nitrate due to lack of information on both cooking technique and doneness level. Although many studies ask about the consumption of well-done meat, one needs information on both cooking methods and doneness levels to get accurate intake estimate. Studies in different populations need specific databases relevant for their consumption patterns (e.g., Japan, Sweden, and U.S.). The Computerized Heterocyclic Amines Resource for Research in Epidemiology of Disease (CHARRED) software application was specifically developed for the U.S. population, therefore, appropriate for our study [3].

We conducted two nested case-control studies within the Prostate, Lung, Colorectal, and Ovarian (PLCO) Cancer Screening Trial to examine the effect of meat-related mutagens and their interaction with 513 tag SNPs from 18 selected genes involved in phase I (cytochrome P450s (CYP)) and phase II metabolism (sulfotransferases (SULT), N-acetyltransferases (NAT), UDP-glucuronosyltransferases (UGT), and glutathione S-transferases (GST)) in relation to both colorectal adenoma and colorectal cancer risk.

\section{METHODS}

\section{Study population}

The PLCO Cancer Screening Trial is a randomized, multi-center clinical trial investigating the efficacy of screening for prostate, lung, colorectal, and ovarian cancer [28, 29]. Participants aged 55-74 years were recruited from 10 centers in the United States from 1993 to 2001 
and randomly assigned to the screened or control arm of the trial. The study was approved by the Institutional Review Board at the National Cancer Institute and the ten study centers, and all participants provided written informed consent. The present investigation is restricted to the individuals in the screening arm of the trial $(n=77,469)$ who completed a self-administered risk factor questionnaire at baseline, filled out a food frequency questionnaire (FFQ) with detailed meat cooking questions, and provided a blood sample.

We conducted two separate nested case-control studies with two disease outcomes: a) advanced colorectal adenoma; and b) colorectal cancer. To be eligible for these analyses, participants had to have no history of cancer prior to completion of the FFQ, and no prior history of self-reported colon disease (Crohn's disease, ulcerative colitis, familial polyposis or Gardner's syndrome). Individuals with a prior history of colorectal polyps were also excluded from the analyses of adenoma.

\section{a) Advanced Adenoma Sample}

Adenoma cases were participants found to have at least one advanced colorectal adenoma ( $\geq 1 \mathrm{~cm}$ in size, containing villous/tubulovillous characteristics, or had severe dysplasia) of the distal colon or rectum at baseline. Controls were subjects who underwent a successful sigmoidoscopy exam (defined as insertion to at least $50 \mathrm{~cm}$ with $\geq 90 \%$ of mucosa visible or suspicious lesion found) at baseline and had no evidence of a left-sided polyp. Controls were matched to cases on gender, ethnicity, and for a subset, age. A total of 1,243 advanced adenoma cases and 1,419 controls with DNA were available for this study.

\section{b) Colorectal Cancer Sample}

Colorectal cancer cases were identified through self-report from the annual study update questionnaire, death certificates, or physician reports, and confirmed by review of pathology reports and medical records. Cases were identified through December 31, 2006. Controls were subjects without a diagnosis of colorectal cancer at the time the case was diagnosed, matched on age, gender, ethnicity, and year of randomization. A total of 371 cases and 405 controls with DNA were identified and genotyped in this study Five cases from the adenoma sample later on developed colorectal cancer and were also included in the cancer sample.

\section{Dietary Assessment}

Participants completed a 137-item FFQ with a detailed meat-cooking module that ascertained usual diet during the previous 12 months. Most (89\%) study participants completed the FFQ prior to or on the same day as the baseline sigmoidoscopy. Using the CHARRED (www.charred.cancer.gov) software application [3], we generated intake estimates of 3 HCAs (ng/day): 2-amino-3,8-dimethylimidazo[4,5-f]quinoxaline (MelQx), 2amino-3,4,8-trimethylimi-dazo[4,5-f]quinoxaline (DiMelQx) and 2-amino-1-methyl-6phenyl-imidazo[4,5-b]pyridine (PhIP), as well as benzo[a]pyrene (B[a]P), which is a marker of $\mathrm{PAH}$ exposure. We estimated nitrate and nitrite from processed meats using a nitrate/nitrite database based on laboratory measured values from 10 types of processed meat samples (bacon; sausage; hot dogs; roast beef; pork chops; ham; lunch meats, including bologna, salami and processed ham) that represented $90 \%$ of the processed meat consumed in the United States $[3,17]$.We multiplied the frequency of consumption of each processed meat item by the portion size and by the nitrate or nitrite concentration of the 
respective item to estimate nitrate and nitrite intake $(\mathrm{mg} /$ day). Participants were excluded from these analyses if they had $\geq 8$ missing or invalid responses of the FFQ or missing data on the meat mutagens ( $n=71$ and $n=18$ for the adenoma and cancer samples, respectively), leaving 1,386 advanced adenoma cases (171 rectal, 462 distal colon, 566 multiple adenoma (either side), and 6 with unknown location) and 1,205 controls, and 364 cancer cases (182 proximal colon, 102 distal colon, 79 rectal, 1 unknown location), and 394 controls for analyses.

\section{Genotyping}

A total of 513 tag SNPs were selected for 18 genes or gene regions directly involved in the metabolism of HCAs, PAHs, or possibly with NOCs (Aromatic hydrocarbon receptor (AHR), CYP1A1/CYP1A2, CYP1B1, CYP2A6, CYP2C9, CYP2D6, CYP2E1, Epoxide hydrolase (EPHX1), GSTA1, GSTM1, GSTM3, GSTP1, GSTT1, NAT1, NAT2, NAD(P)H dehydrogenase (quinone 1)(NQO1), SULT1A1/SULT1A2, and the UGT1A locus). Tag SNPs were selected including the region $20 \mathrm{~kb}$ upstream and $10 \mathrm{~kb}$ downstream of the genes, using the CEU, JPT, CHB, and YRI HapMap populations and the Carlson method [30] as implemented in Tagzilla with a $\mathrm{r} 2$ threshold of 0.8 and minor allele frequency (MAF) $\geq 5 \%$. SNPs with known or putative functional significance (i.e. nonsynonymous, promoter, intron-exon splice sites) were also included whenever possible. The SNPs from the Phase I and II genes for putative pathway for metabolism of HCAs, PAHs and nitrites/nitrates (Supplemental Figure 1 [11, 31, and 32]) were genotyped on a custom iSelect panel utilizing Illumina's Infinium platform.

Whole blood or buffy coat DNA was extracted with QIAamp DNA Blood Midi or Maxi Kits. For quality control purposes, replicate samples from 195 individuals ( 7\% of the population) were interspersed randomly within the plates. Genotyping was conducted at the National Cancer Institute Core Genotyping Facility, National Institutes of Health. We required all cases and controls to have a $90 \%$ call rate for analysis. The overall concordance rate was $>99 \%$ for replicated samples. We excluded SNPs with a call rate $<90 \%$, MAF $<1 \%$ in the adenoma set and 5\% in the cancer set (due to lower power), or Hardy-Weinberg Equilibrium p-value < 1 x 10-6 among Caucasian controls. Of the 513 SNPs we selected, 380 SNPs remained for the adenoma analyses, and 325 SNPs remained for the cancer analyses. There were a few highly correlated SNPs among the Caucasians in our study as the tag SNPS were selected for multiple populations. SNPs with an r2>0.95 among Caucasians were considered to be equivalent for interaction testing.

\section{Statistical Analysis}

All analyses were conducted separately for the prevalent colorectal adenoma and colorectal cancer study populations. Differences in baseline characteristics between cases and controls were assessed using chi-square tests for categorical variables, such as sex, and $t$ tests for continuous variables, such as age. Due to a skewed intake distribution of the meat mutagens, we transformed these variables prior to performing t-tests, using the inverse rank transformation.

\section{Main Effects: Meat Mutagens and SNPS}

In order to confirm what has been previously reported in PLCO subgroup analyses, we examined the main effects of each mutagen and SNP on colorectal adenoma and cancer risk using unconditional multivariable logistic regression. The main effects of the meat 
mutagens were adjusted for all relevant covariates (listed as footnotes in the tables). Meat mutagens were categorized as low $\left(0-39^{\text {th }}\right.$ percentile), medium $\left(40-79^{\text {th }}\right.$ percentile), and high ( $\geq 80^{\text {th }}$ percentile) intake, as the distribution of these mutagens is highly skewed with the majority of individuals consuming small amounts, and previous analyses, both within the PLCO study population and within other studies, have identified the top quintile as potentially the most important with regard to cancer risk [33, 34] (e.g., PhIP intake in adenoma dataset: quintile 1 : range $=0-19.5 \mathrm{mg} / \mathrm{d}$, median $=10.1 \mathrm{mg} / \mathrm{d}$; quintile 5 : range $=$ $166-3069 \mathrm{mg} / \mathrm{d}$, median $=268 \mathrm{mg} / \mathrm{d}$ ). The main effects of the SNPs were examined using PLINK, a whole genome association analysis toolset [35] assuming a log-additive model for the genotype, and were adjusted for age, sex, and ethnicity. Results were adjusted for multiple testing using the False Discovery Rate (FDR) [36]. We also used a Bonferroni correction for the total number of tag SNPs for each individual gene or gene region (genebased correction).

\section{Gene $\times$ Environment (GxE) Interactions}

Because the experimental literature on the role of XME genes in relation to HCA, PAH and NOC metabolism is far from complete, we did not restrict our analyses to those genemutagen interactions previously reported, but rather used an exploratory approach when testing for interactions between the all SNPs and mutagens under study. We used a twostep test for G×E interactions as described by Murcray et al.[37], to identify SNPs involved in a GXE interaction. In the first step, we examined the association of each SNP with each mutagen in a linear regression model among cases and controls combined, adjusting for age, sex, and ethnicity. Due to a skewed intake distribution, we transformed the exposure variables using the inverse normal rank transformation. Linear regressions were calculated using PLINK.

The subset of $\mathrm{m}$ SNPs that exceeded the significance threshold of $P<0.10$ was taken forward to Step 2 for the actual case-control test for a GxE interaction. In Step 2, an unconditional logistic regression model, assuming a dominant genetic model, adjusting for age, sex, and ethnicity, was calculated with a multiplicative interaction term of the SNP with the respective meat mutagen (categorical variable assigning median values), as well as the individual main effect terms. Further adjustment for all confounders selected in the mutagen main effects analyses did not change the results appreciably. Significance at this step was defined as a P-value $<\alpha / m$, where $\alpha$ was the desired overall Type I error rate of $10 \%$. When multiple SNPs from the same gene exceeded the $P<0.10$ threshold level, a Meff was calculated to determine the effective number of independent comparisons [38].

All interactions meeting the significance threshold of $P<0.05$ were further evaluated using logistic regression models to examine the association between the categorical meat mutagen intake and colorectal adenoma or cancer, stratified by genotype assuming a dominant model. We also conducted stratified analyses by ever and never smokers, rectal and non-rectal adenomas, and single versus multiple adenomas. The top interactions in the cancer set were also examined by proximal, distal and rectal cancer. Models for Step 2 as well as the models for the association of the meat mutagens and colorectal adenoma or cancer were calculated using STATA version 9.0. 


\section{RESULTS}

A total of 1,205 cases and 1,386 controls were available for the advanced colorectal adenoma analysis and 364 cases and 394 controls were included in the colorectal cancer population (Table 1). Over $90 \%$ of the study subjects were Caucasian and over $60 \%$ were males; in the adenoma study, cases were older than controls.

Table 1: Distribution of baseline characteristics in nested case control studies of advanced colorectal adenoma and colorectal cancer in the PLCO Cancer Screening Trial.

\begin{tabular}{|c|c|c|c|c|c|c|}
\hline \multirow[b]{2}{*}{ Characteristic } & \multicolumn{2}{|l|}{ Adenoma } & \multirow[b]{2}{*}{$P$} & \multicolumn{2}{|l|}{ Cancer } & \multirow[b]{2}{*}{$P$} \\
\hline & $\begin{array}{l}\text { Cases } \\
(\mathrm{n}=1205)^{\mathrm{a}}\end{array}$ & $\begin{array}{l}\text { Controls } \\
(\mathrm{n}=1386)^{\mathrm{a}}\end{array}$ & & $\begin{array}{l}\text { Cases } \\
(\mathrm{n}=364)^{\mathrm{a}}\end{array}$ & $\begin{array}{l}\text { Controls } \\
(\mathrm{n}=394)^{\mathrm{a}}\end{array}$ & \\
\hline Age, years & $63.1 \pm 5.2$ & $62.6 \pm 5.3$ & 0.02 & $67.6 \pm 6.5$ & $67.5 \pm 6.3$ & 0.81 \\
\hline Sex, n (\%) & & & 0.83 & & & 0.71 \\
\hline Male & 776 (64.4) & $887(64.0)$ & & $216(59.3)$ & 239 (60.7) & \\
\hline Female & $429(35.6)$ & 499 (36.0) & & $148(40.7)$ & $155(40.3)$ & \\
\hline Ethnicity, n (\%) & & & 0.23 & & & 0.97 \\
\hline Non-Hispanic white & $1136(94.3)$ & $1284(92.6)$ & & 311 (90.9) & 357 (90.6) & \\
\hline Non-Hispanic black & $29(2.4)$ & $46(3.3)$ & & $18(5.0)$ & $21(5.3)$ & \\
\hline Other & $40(3.3)$ & $56(4.0)$ & & $15(4.1)$ & $16(4.1)$ & \\
\hline $\begin{array}{l}\text { First degree family history } \\
\text { of colorectal cancer, n (\%) }\end{array}$ & $157(13.1)$ & $136(9.9)$ & 0.01 & $56(15.6)$ & $45(11.6)$ & 0.11 \\
\hline \multicolumn{7}{|l|}{ Smoking status, n (\%) } \\
\hline Never & $462(38.4)$ & $657(47.4)$ & $<0.01$ & $157(43.1)$ & 164 (41.6) & 0.90 \\
\hline Former cigarette smoker & $165(13.7)$ & $94(6.8)$ & & $167(45.9)$ & 187 (47.5) & \\
\hline Current cigarette smoker & $577(47.9)$ & $635(45.8)$ & & $40(11.0)$ & $43(10.9)$ & \\
\hline \multicolumn{7}{|c|}{$\mathrm{B}[\mathrm{a}] \mathrm{P}$ (ng/day) } \\
\hline median (IQR) & $7.0(1.3,36.1)$ & $6.9(1.2,35.9)$ & 0.17 & $5.5(1.2 .23 .8)$ & $5.4(1.0,36.4)$ & 0.74 \\
\hline \multicolumn{7}{|l|}{ PhIP (ng/day) } \\
\hline median (IQR) & $65.4(24.6,132.6)$ & $61.1(23.3,132.3)$ & 0.69 & $50.0(21.0 .127 .5)$ & $53.8(22.8,135.8)$ & 0.23 \\
\hline \multicolumn{7}{|l|}{ MelQx (ng/day) } \\
\hline median (IQR) & $22.7(9.75,43.2)$ & $20.5(8.94,41.8)$ & 0.20 & $18.0(9.1 .38 .7)$ & $18.9(9.0,39.6)$ & 0.59 \\
\hline \multicolumn{7}{|l|}{ DiMelQx (ng/day) } \\
\hline median (IQR) & $1.02(0.23,2.47)$ & $0.95(0.27,2.2)$ & 0.39 & $0.80(0.18,2.22)$ & $0.90(0.22,2.22)$ & 0.35 \\
\hline \multicolumn{7}{|l|}{$\begin{array}{l}\text { Combined nitrate and } \\
\text { nitrite (ng/day) }\end{array}$} \\
\hline median (IQR) & $0.32(0.13,0.61)$ & $0.28(0.12,0.59)$ & 0.10 & $0.31(0.13,0.61)$ & $0.28(0.12,0.55)$ & 0.36 \\
\hline
\end{tabular}

\section{Main Effects: Meat Mutagens}

Consistent with a previous analysis of prevalent adenoma in the PLCO trial [34], there was an elevated risk of colorectal adenoma in the top quintile of MelQx intake when compared to the bottom quintile (OR=1.17, 95\% Cl: 0.94-1.45); however, in the current study population with $\sim 2000$ fewer adenoma cases, the risk did not reach statistical significance and was attenuated after adjustment for confounders (Supplemental Table 1). We observed a borderline significant positive association between nitrate and nitrate intake from meat and adenoma risk ( $P$-trend $=0.05)$, but this was attenuated with covariate adjustment. B[a]P, PhIP and DiMelQx intake were not associated with adenoma risk in this population. Furthermore, none of the meat mutagens were associated with colorectal cancer. Due to subjects with missing values, the number of cases and controls in the 
multivariable models were lower (adenoma: 1,183 cases, 1,358 controls; cancer: 387 cases, 358 controls) than in the age, ethnicity and sex adjusted model, however, this did not explain the observed attenuation. The ORs for MelQx and colon cancer in particular changed considerably after adjustment for confounders; level of education and total daily energy intake contributed the most to this change.

\section{Main Effects: SNPs}

We found that SNPs from the UGT1A, EPHX1, NAT1 genes were nominally associated with both colorectal adenoma ( $n=11$ SNPs) and cancer risk ( $n=20$ SNPs) $(P<0.05)$ (Supplemental Table 2). In addition, one SNP from the GSTA1 locus was associated with adenoma risk, whereas one SNP at the NQO1 gene was associated with colorectal cancer. After adjusting for multiple testing for all the SNPs tested using the FDR, none of these findings remained statistically significant in either the adenoma or cancer analysis. However, UGT1A rs 7569014 remained associated with colorectal cancer risk at $P \leq 0.05$ after the gene-based multiple testing correction.

\section{Gene $\times$ Environment Interactions}

A schematic representation of the results from the two-step test for G×E interaction for both the adenoma and cancer study populations is presented in Figure 1. The subset and number of SNPs that exceeded the significant threshold of $P<0.10$ in Step 1, differed by outcome and mutagen under study. SNPs that passed this initial screening step were taken forward to the actual case-control test of GxE interaction analyses in Step 2.

\section{a) Advanced Prevalent Adenoma}

The GxE interaction analyses performed in Step 2 yielded fifteen interactions with $\mathrm{P} \leq 0.10$ between $\mathrm{B}[\mathrm{a}] \mathrm{P}, \mathrm{HCAs}$ and several SNPs, including those in the GSTM3, UGT1A, AHR, EPHX, NAT1 and CYP2C9 gene regions (Table 2, Figure 1). The most significant interaction was between NAT1 (rs6586714) and MelQx ( $P$-interaction $=0.001$ ) which was the only finding that remained significant after adjustment for multiple testing. Various SNPs from the UGT1A locus appeared to modify the association between B[a]P (3 SNPs; $r>0.79$ ), PhIP (4 SNPs; $r-0.12-0.89)$, and MelQx intake (1 SNP) and adenoma risk $(P<0.10)$. Two SNPs ( $r=0.39$ ) located within the EPHX1 region ( $r$ 2671272 and $r s 868966)$ appeared to modify the effect of PhIP and DiMelQx on colorectal adenoma risk $(P \leq 0.05)$.

We further explored the risk pattern for all interactions that met the unadjusted significance threshold of 0.05. Age, ethnicity and sex adjusted associations between mutagen intake and colorectal adenoma stratified by genotype are presented in Table $\mathbf{3}$. There was an increased risk of colorectal adenoma with increasing intake of MelQx among individuals carrying a GG genotype for NAT1 rs6586714 (OR=1.43, 95\% Cl: 1.11-1.85 for high intake compared to low intake; $P$-trend=0.007), whereas the risk was decreased among GA and AA carriers (OR= 0.50 for high intake compared to low intake, $95 \% \mathrm{Cl}: 0.30$ 0.84; $P$-trend=0.01; Table 3). Increased intake of DiMelQx was positively associated with adenoma risk in individuals carrying an AA genotype for $E P H X 1$ rs868966 (OR=1.45, 95\% Cl: 0.98, 2.15; $P$-trend=0.06). 


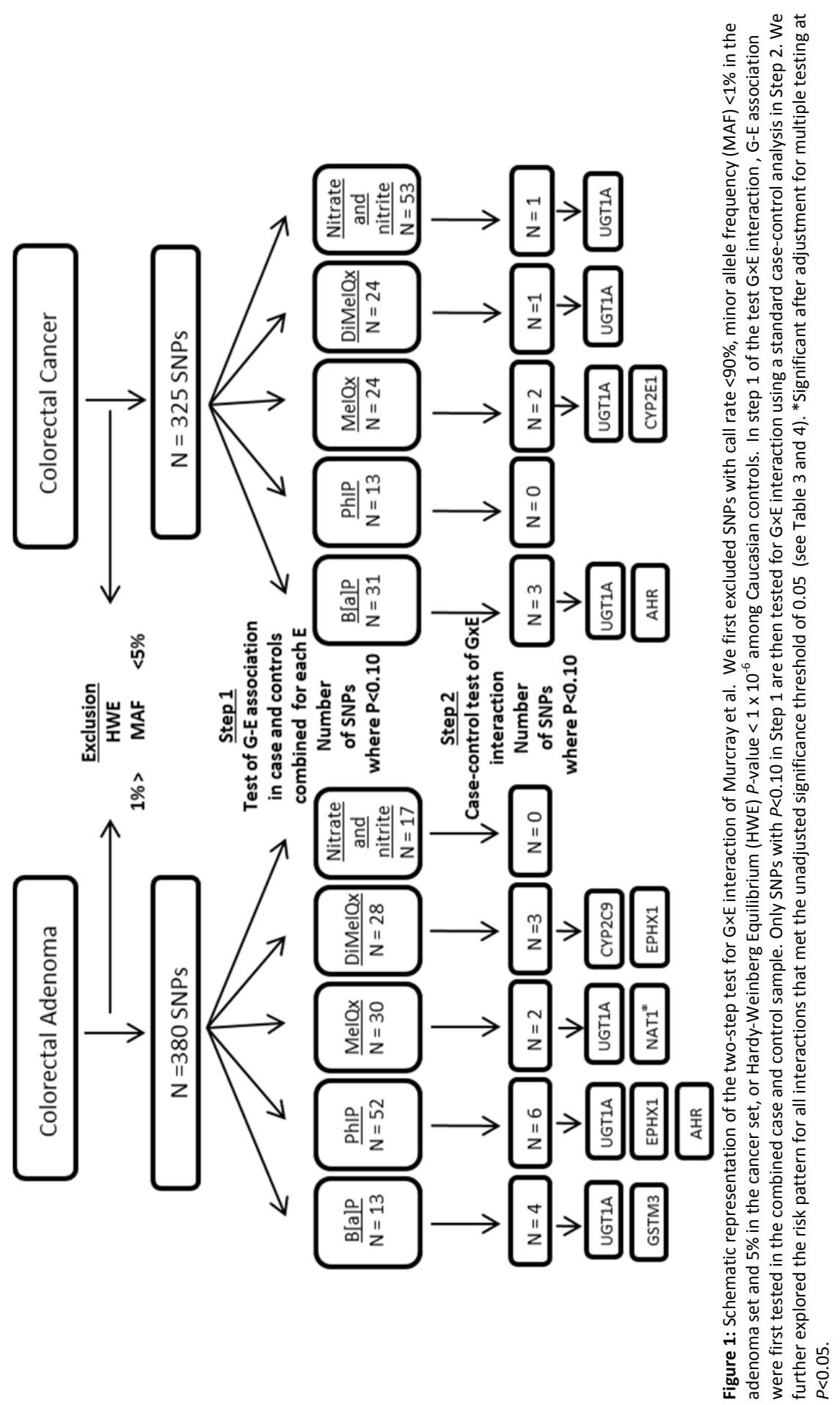


Table 2: Meat mutagen-SNP interaction and risk of colorectal adenoma and cancer for all interactions with $P \leq 0.10$

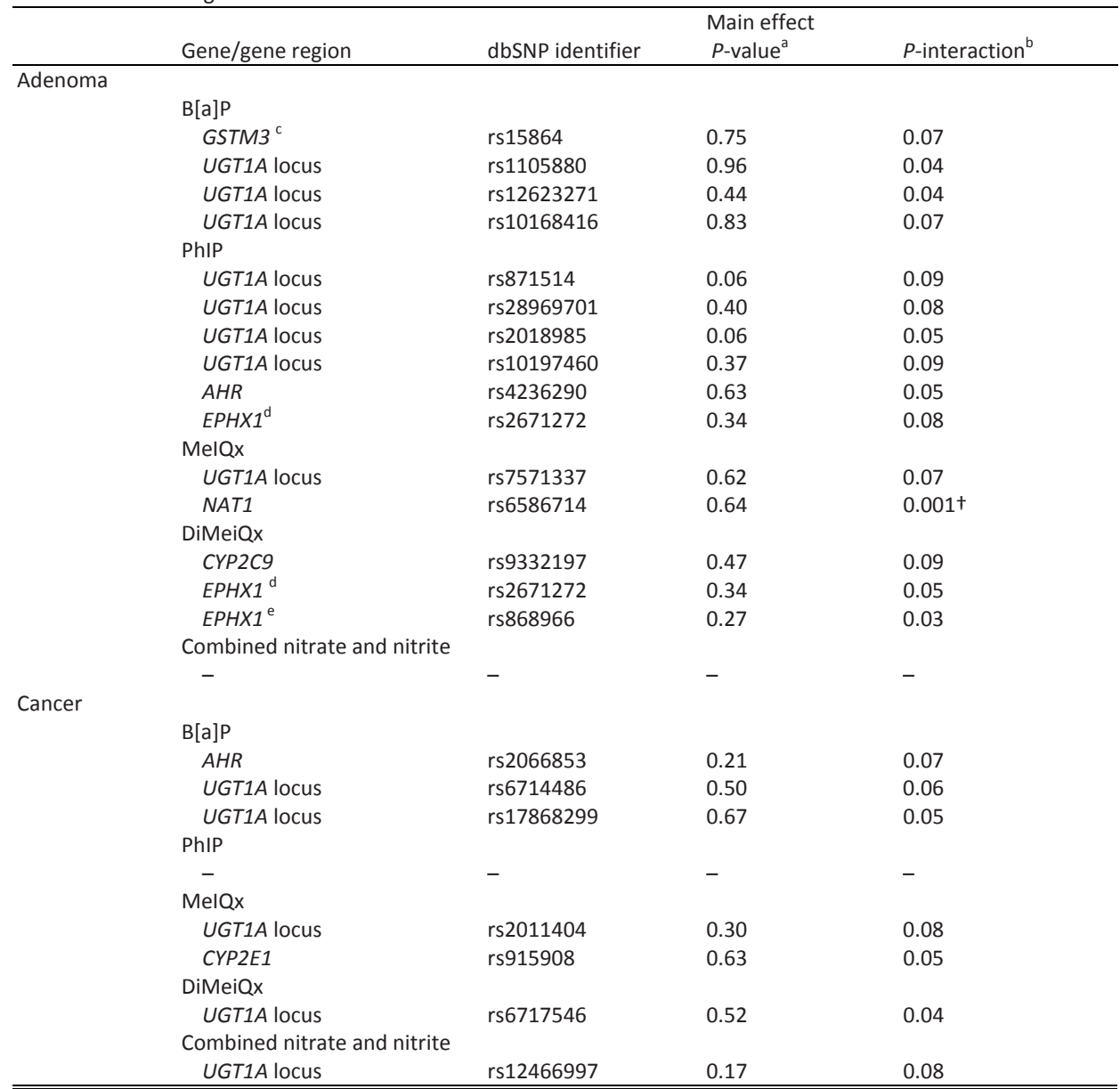

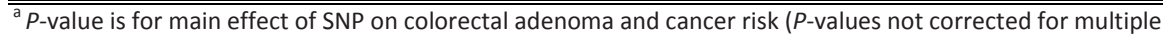
testing). Adjusted for age, sex and ethnicity and assuming a log-additive model.

${ }^{\mathrm{b}} P$-value is for a test for interaction using the likelihood ratio test comparing models with and without the crossproduct of SNP for the given gene and the median of the categories of meat mutagen intake. $P$-values not corrected for multiple testing. Adjusted for age, sex and ethnicity and assuming a dominant model.

${ }^{c}$ SNP located within $10 \mathrm{~kb}$ downstream of EPS8L3.

${ }^{\mathrm{d}} \mathrm{SNP}$ located within $10 \mathrm{~kb}$ downstream of TMEM63A.

e SNP located within $10 \mathrm{~kb}$ upstream of LEFTY3.

+ Statistically significant at $P<0.05$ after adjustment for multiple testing. 


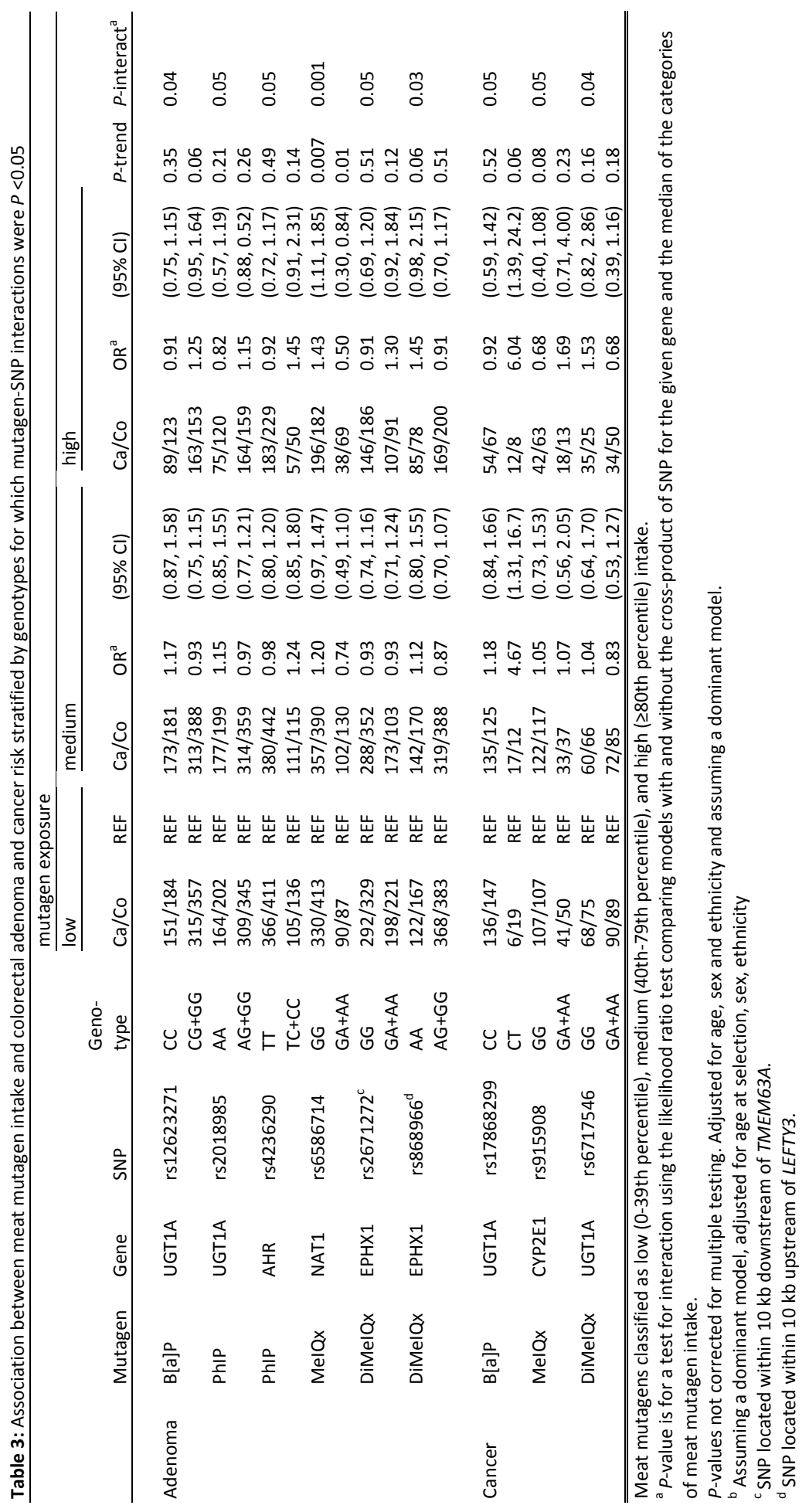


We additionally ran the GxE interaction analyses for total mutagenic activity, a measure incorporating mutagenicity of all meat-related mutagens. We found five interactions in the adenoma set, whereas one interaction (rs28969701- UGT1A locus) was observed in the cancer data. However, these SNPs came from genes similar as those interacting with the individual mutagens (CYP2C9, EPHX1, GSTM3, UGT1A locus) (data not shown). The correlation between total mutagenic activity and the individual HCAs and PAHs for adenomas was highest for MelQx ( $r=0.84)$, followed by PhIP ( $r=0.78)$, DiMelQx ( $r=0.76)$, and $B[a] P(r=0.51)$.

Subgroup analyses indicated that the top interactions were similar for the subgroups of any rectal $(n=377)$ and any distal adenoma $(n=957)$ and single $(N=639)$ versus multiple adenomas $(n=566)$. However, the interaction between PhIP intake and rs2018985 was only observed with respect to multiple adenomas but not for single adenomas (data not shown). Moreover, our top interaction were similar in direction and magnitude for ever- $(n=743)$ and never $(n=462)$ smokers, except for the interaction between DiMelQx and rs868966 which was only observed among ever smokers (data not shown).

\section{b) Colorectal Cancer}

In the carcinoma dataset, seven interactions with $P \leq 0.10$ were observed (Table 2, Figure 1 ). We found a suggestive interaction between several SNPs in the UGT1A region and intake of B[a]P (rs6714486, rs17868299), MelQx (rs2011404), DiMelQx (rs6717546), and combined nitrate and nitrite from meat ( $r$ 12466997). The association between MelQx and colorectal cancer risk seemed to be modified by a SNP in the CYP2E1 gene ( $r$ 915908) ( $\mathrm{P}$-interaction = 0.05). However, neither these nor other interactions for colorectal cancer were deemed significant after adjusting for multiple testing.

Age, ethnicity and sex adjusted associations between mutagen intake and colorectal cancer stratified by genotype are presented in Table 3 (SNPs with P-interaction $\leq 0.05$ ). High consumption of $\mathrm{B}[\mathrm{a}] \mathrm{P}$ was associated with an increased risk of colorectal cancer among individuals carrying the CT genotype for UGT1A rs17868299 (OR=6.04 for high intake compare to low intake; $95 \% \mathrm{Cl}$ : $1.39-24.2 ; P$-trend $=0.06)$. A suggestive inverse association between MelQx intake and colorectal cancer risk was observed among the wild-type variant of rs915908 (CYP2E1) (OR=0.68 for high intake compared to low intake, $95 \% \mathrm{Cl}$ : $0.40-1.08 ; P$-trend $=0.08$ ).

Interactions between SNPs in the UGT1A locus and various meat mutagens were observed for both the adenoma and cancer endpoints. No strong correlation $(r 2<0.25)$ between SNPs found in the adenoma and cancer analyses were found (results not shown). However, the interaction observed between rs7571337 and MelQx intake for adenoma could be replicated for the cancer endpoint $(P$-interaction=0.09), and the ORs for the SNP-stratified results were in the same direction, though attenuated. The pooled $\mathrm{OR}(95 \% \mathrm{Cl})$ combining the adenoma and cancer results for high intake compared to low intake was 1.72(1.032.39) for the TT genotype and $0.95(0.70-1.13)$ for the TC+CC genotype. Nevertheless, all other interactions that were significant in the adenoma dataset were not found to be so in the cancer study $(P>0.10)$, and vice versa (Supplemental Table 3A and 3B). 
The OR and $95 \% \mathrm{Cl}$ for the main effects of the SNPs that were found to be involved in a GxE interaction $(P<0.10)$ are presented in Supplemental Table 4. Two SNPs from the UGT1A locus (rs rs871514 and rs2018985) were marginally associated with colorectal adenoma $(P=0.06)$, but did not remain statistically significant after adjustment for multiple testing using either the FDR or the gene-based approach.

We examined the top interactions (as described in Tables $3 \mathrm{~A}$ and $3 \mathrm{~B}$ ) by proximal, distal and rectal cancer sub site and observed that the interaction effect for rs6717546 remained present in all endpoints. However, the interaction between B[a]P and rs17868299 was only observed in the distal colon, and the magnitude of the SNP stratified associations for the variant allele was larger than that in the combined group of cancers (CC genotype: ORmedium=4.36 95\% $\mathrm{Cl} 1.02-18.55$, ORhigh=8.82 95\% Cl 1.60-48.72) (data not shown); however, since the numbers were small, this may be a chance finding.

\section{DISCUSSION}

We observed that the effect of MelQx intake on advanced colorectal adenoma risk differed by NAT1 genotype. Additional evidence of effect modification of the association between various meat-related HCAs, PAHs, and nitrate/nitrite and advanced colorectal adenoma and cancer by variants in UGT1A, CYP2E1, EPHX1, AHR, and GSTM3 was observed; however, correction for multiple comparisons indicated that these observations may be due to chance, despite that this the largest and most comprehensive study to date.

Our finding that NAT1 modified the effect of MelQx on the risk of advanced colorectal adenoma is in agreement with results from animal and in vitro research. NAT1 is primarily expressed in hepatic and colonic tissue and has been extensively shown to be an important enzyme in the bio-activation of multiple HCAs via O-acetylation processes [39]. NAT1 enzyme activity is characterized by strong between population heterogeneity that likely results in inter-individual differences in acetylation rates [40]. Functionality of the variant observed in our analyses (rs6586714), which is located in the intron region of NAT1, is lacking and not strongly correlated with any known functional NAT1 alleles in the iSelect panel $(\mathrm{r} 2<0.20)$. One previous analysis studied the interaction between various NAT1 alleles $(* 3, * 4, * 10, * 11$; categorizing participants into slow, normal and fast acetylaters) and meat intake in relation to colorectal adenoma risk but reported null results [41]. Nevertheless, evidence from the few epidemiological studies that examined interactions between NAT1 and meat $[15,23,24]$ or HCA intake [42] and colorectal cancer risk, points towards an increased risk among rapid acetylaters. However, these findings may have bias towards the null due to the generally small study samples, lack of detailed HCA exposure information and the debatable classification of NAT1 alleles. From our prior research, there is evidence that MelQx is estimated with greater accuracy than other HCAs [43]. In addition, although MelQx is consumed at lower levels in the diet, it is a more potent mutagen than PhIP is [44]. Moreover, it could be that this interaction is observed due to linkage disequilibrium with another variant in the region that has an effect on a neighboring gene.

Although we observed some suggestive modifying effects in SNPs in other XME genes, the findings were not significant after adjusting for multiple testing. For example, multiple UGT1A polymorphisms were suggestively associated with colorectal adenoma and cancer 
or appeared to interact with meat mutagens and tumor risk. UGTs are phase II conjugation enzymes that are primarily expressed in the luminal cells of the gastrointestinal tract, and have been implicated in the detoxification of HCAs and B[a]P [45, 46]. Previous epidemiological studies suggest that alterations in UGT genes may indeed influence the elimination of meat specific mutagens; one case-control reported an interaction between $\mathrm{B}[\mathrm{a}] \mathrm{P}$ intake and variants in UGT1A1 promoter polymorphisms at position -53 and -3156 [47], whereas a suggestive interaction between DiMelQx and UGT1A7 [48] acetylation status was reported in a different study. However, the multiple SNPs located across the UGT1A region that were involved in interactions with specific meat mutagens in the present study were found in overlapping intron regions of UGTA1 genes (UGT1A3 - A10), and mostly highly correlated, making it impossible to differentiate the associations of each statistically. Future research is needed to unravel this linkage disequilibrium and focus on individual UGT1A isoenzymes to characterize their etiological relevance with meat mutagen induced colorectal carcinogenesis.

We found some other suggestive interactions between SNPs in the CYP2E1, AHR, and EPHX1 gene, although results after multiple testing suggests these findings may be due to chance. Although these genes are implicated in the metabolism of meat mutagens, there is little evidence in the literature for the functionality of the observed SNPs. However rs2066853 (AHR) encodes for an amino acid change in the protein (Arg554Lys), whereas rs15864 (GSTM3) is correlated ( $\mathrm{r} 2=0.81$ ) with $\mathrm{rs7483}$, a missense mutation in the same gene responsible for an amino acid substitution (Val224lle) that significantly alters enzyme activity [49].

Despite the biological rationale for interactions between meat specific mutagens and polymorphisms in phase I and II XME genes in relation to colorectal carcinogenesis, the epidemiological evidence remains inconclusive. This lack of consistency may be due to inadequate sample size, the high potential for chance findings as a result of multiple comparisons, and the over-interpretation of subgroup findings in many gene-environment interaction studies. Although the present study is the largest and most comprehensive to examine these associations to date, we were still underpowered to detect modest effects of single gene variants, especially for the colorectal cancers. To gain power, we applied a two-step approach [37], incorporating a preliminary screening step to identify the SNPs involved in possible gene-environment interactions, and hence protect against false positive results. Moreover, the iSelect genotyping panel was specifically designed to capture only tagging SNPs from a priori selected genes involved in carcinogen metabolism to reduce the number of tests performed.

Examination of the same set of SNPs and mutagens yielded different interactions in the adenoma and cancer analyses. Although it may be that some of our findings are false positives, the differences we observed may reflect variation in gene-environmental interactions between initiation and progression in the carcinogenesis process. There is tremendous heterogeneity among colorectal adenomas, with only a small subset of adenomas progressing to cancer. Therefore, interactions related to the formation of adenoma may not be observed for cancer if they do not also stimulate progression to cancer. In contrast, interactions associated with malignant transformation may only be observed for cancer. Nevertheless, we did observe multiple interactions between SNPs at 
the UGTA1 locus and both colorectal adenoma and cancer, suggesting that this locus may be important for progression and transformation. Many of the XME genes under study are part of larger multigene families. As a result there could be redundancy among these enzymes, such that if the activity of one is modified as a result of a specific genetic variation, others may continue to carry out the metabolism of meat mutagens. We were not able to examine differences in etiology between proximal and distal adenomas and advanced and non-advanced adenomas; the present study was restricted to advanced distal and rectal colorectal adenomas only.

We made use of a comprehensive assessment of meat cooking methods that enabled detailed estimation of HCAs, PAHs, and nitrate/nitrite, but this is nonetheless susceptible to measurement error. We did not collect information concerning all aspects of meat cooking methods that could have influenced carcinogen production, such as microwaving prior to cooking [50], marinating the meat[51], or flipping burgers more often [52]. Moreover, during meat cooking, numerous other mutagenic compounds could be formed that may be correlated with the mutagens under study, and could thus potentially explain the observed interactions (e.g. between NAT1 and MelQx). Our manuscript focuses on meat carcinogenesis and, therefore, we did not consider other potential dietary sources of nitrite and nitrate. Although the majority of dietary nitrite comes from processed meats [53], other food items and drinking water may contribute to intake.

Given that this study was nested within the screening arm of the PLCO Cancer Screening Trial, we examined possible interactions using colorectal adenomas as an endpoint, largely asymptomatic precursors of colorectal cancer. Moreover, selection and surveillance bias is minimal since both cases and controls had an equal opportunity to have a colorectal adenoma or cancer detected. Our colorectal cancer analyses were based on a prospective study of incident cases, eliminating the potential for recall bias. However, inherent to the nature of the study population, participants were generally more educated, less likely to smoke, more physically active and more likely to be Caucasian than the general US population, thereby limiting the generalizability of the study results. All adenoma cases and controls and the majority of cancer cases and controls underwent at least one sigmoidoscopy screening as part of the trial. Since nearly all polyps found were subsequently removed in this heavily screened population, it is possible that the colorectal cancers observed were somehow different than what would be observed in the general population, perhaps more likely to arise de novo. It is also possible that some subjects changed their diet following screening. However, this is unlikely to have affected the results of this study since diet was assessed prior to screening and cancer cases were diagnosed a median of 3.2 years later, which is shorter than the predicted time span (10-20 years) of the adenoma-carcinoma sequence.

In conclusion, we found some evidence that common variants in XME genes may modify the association between meat mutagens and colorectal neoplasia. The strongest evidence for an interaction was observed between MelQx intake and a NAT1 polymorphism in relation to colorectal adenoma risk. Future pooled initiatives that would have larger sample sizes should further evaluate this complex interplay to better understand these relationships. 


\section{REFERENCES}

1. World Cancer Research Fund/American Institute for Cancer Research, Food, Nutrition, Physical Activity, and the Prevention of Cancer: a Global Perspective. 2007, AICR: Washington DC.

2. Cross, A.J. and R. Sinha, Meat-related mutagens/carcinogens in the etiology of colorectal cancer. Environ Mol Mutagen, 2004. 44(1): p. 44-55.

3. Sinha, R., et al., Development of a food frequency questionnaire module and databases for compounds in cooked and processed meats. Mol Nutr Food Res, 2005. 49(7): p. 648-55.

4. Hughes, R., et al., Dose-dependent effect of dietary meat on endogenous colonic N-nitrosation. Carcinogenesis, 2001. 22(1): p. 199-202.

5. Ochiai, M., et al., Induction of intestinal tumors and lymphomas in C57BL/6N mice by a food-borne carcinogen, 2-amino-1-methyl-6-phenylimidazo[4,5-b]pyridine. Jpn J Cancer Res, 2002. 93(5): p. 47883.

6. Sugimura, T., Overview of carcinogenic heterocyclic amines. Mutat Res, 1997. 376(1-2): p. 211-9.

7. Ito, N., et al., A new colon and mammary carcinogen in cooked food, 2-amino-1-methyl-6phenylimidazo[4,5-b]pyridine (PhIP). Carcinogenesis, 1991. 12(8): p. 1503-6.

8. Ohgaki, H., S. Takayama, and T. Sugimura, Carcinogenicities of heterocyclic amines in cooked food. Mutat Res, 1991. 259(3-4): p. 399-410.

9. Bogovski, P. and S. Bogovski, Animal Species in which N-nitroso compounds induce cancer. Int J Cancer, 1981. 27(4): p. 471-4.

10. Xue, W. and D. Warshawsky, Metabolic activation of polycyclic and heterocyclic aromatic hydrocarbons and DNA damage: a review. Toxicol Appl Pharmacol, 2005. 206(1): p. 73-93.

11. Shimada, T., Xenobiotic-metabolizing enzymes involved in activation and detoxification of carcinogenic polycyclic aromatic hydrocarbons. Drug Metab Pharmacokinet, 2006. 21(4): p. 257-76.

12. Yeh, C.C., et al., Polymorphisms of cytochrome P450 $1 \mathrm{~A} 2$ and N-acetyltransferase genes, meat consumption, and risk of colorectal cancer. Dis Colon Rectum, 2009. 52(1): p. 104-11.

13. Le Marchand, L., et al., Well-done red meat, metabolic phenotypes and colorectal cancer in Hawaii. Mutat Res, 2002. 506-507: p. 205-14.

14. Kampman, E., et al., Meat consumption, genetic susceptibility, and colon cancer risk: a United States multicenter case-control study. Cancer Epidemiol Biomarkers Prev, 1999. 8(1): p. 15-24.

15. Lilla, C., et al., Effect of NAT1 and NAT2 genetic polymorphisms on colorectal cancer risk associated with exposure to tobacco smoke and meat consumption. Cancer Epidemiol Biomarkers Prev, 2006. 15(1): p. 99-107.

16. Chan, A.T., et al., Prospective study of $\mathrm{N}$-acetyltransferase-2 genotypes, meat intake, smoking and risk of colorectal cancer. Int J Cancer, 2005. 115(4): p. 648-52.

17. Ward, M.H., et al., Processed meat intake, CYP2A6 activity and risk of colorectal adenoma. Carcinogenesis, 2007. 28(6): p. 1210-6.

18. Tiemersma, E.W., et al., Effect of SULT1A1 and NAT2 genetic polymorphism on the association between cigarette smoking and colorectal adenomas. Int J Cancer, 2004. 108(1): p. 97-103.

19. Shin, A., et al., Meat intake, heterocyclic amine exposure, and metabolizing enzyme polymorphisms in relation to colorectal polyp risk. Cancer Epidemiol Biomarkers Prev, 2008. 17(2): p. 320-9.

20. Ishibe, N., et al., Genetic polymorphisms in heterocyclic amine metabolism and risk of colorectal adenomas. Pharmacogenetics, 2002. 12(2): p. 145-50.

21. Roberts-Thomson, I.C., et al., Diet, acetylator phenotype, and risk of colorectal neoplasia. Lancet, 1996. 347(9012): p. 1372-4.

22. Goode, E.L., et al., Inherited variation in carcinogen-metabolizing enzymes and risk of colorectal polyps. Carcinogenesis, 2007. 28(2): p. 328-41.

23. Tiemersma, E.W., et al., Meat consumption, cigarette smoking, and genetic susceptibility in the etiology of colorectal cancer: results from a Dutch prospective study. Cancer Causes Control, 2002. 13(4): p. 383-93.

24. Chen, J., et al., A prospective study of $\mathrm{N}$-acetyltransferase genotype, red meat intake, and risk of colorectal cancer. Cancer Res, 1998. 58(15): p. 3307-11.

25. Morita, M., et al., Genetic polymorphisms of CYP2E1 and risk of colorectal cancer: the Fukuoka Colorectal Cancer Study. Cancer Epidemiol Biomarkers Prev, 2009. 18(1): p. 235-41. 
26. Ferrucci, L.M., et al., Xenobiotic metabolizing genes, meat-related exposures, and risk of advanced colorectal adenoma. World Rev Nutr Diet. 101: p. 34-45.

27. Thier, R., et al., Markers of genetic susceptibility in human environmental hygiene and toxicology: the role of selected CYP, NAT and GST genes. Int J Hyg Environ Health, 2003. 206(3): p. 149-71.

28. Prorok, P.C., et al., Design of the Prostate, Lung, Colorectal and Ovarian (PLCO) Cancer Screening Trial. Control Clin Trials, 2000. 21(6 Suppl): p. 273S-309S.

29. Gohagan, J.K., et al., The Prostate, Lung, Colorectal and Ovarian (PLCO) Cancer Screening Trial of the National Cancer Institute: history, organization, and status. Control Clin Trials, 2000. 21(6 Suppl): p. 251S-272S.

30. Carlson, C.S., et al., Selecting a maximally informative set of single-nucleotide polymorphisms for association analyses using linkage disequilibrium. Am J Hum Genet, 2004. 74(1): p. 106-20.

31. Turesky, R.J., The role of genetic polymorphisms in metabolism of carcinogenic heterocyclic aromatic amines. Curr Drug Metab, 2004. 5(2): p. 169-80.

32. Mirvish, S.S., Role of N-nitroso compounds (NOC) and N-nitrosation in etiology of gastric, esophageal, nasopharyngeal and bladder cancer and contribution to cancer of known exposures to NOC. Cancer Lett, 1995. 93(1): p. 17-48.

33. Cross, A.J., et al., A large prospective study of meat consumption and colorectal cancer risk: an investigation of potential mechanisms underlying this association. Cancer Res, 2011. 70(6): p. 2406-14.

34. Sinha, R., et al., Meat, meat cooking methods and preservation, and risk for colorectal adenoma. Cancer Res, 2005. 65(17): p. 8034-41.

35. Purcell, S., et al., PLINK: a tool set for whole-genome association and population-based linkage analyses. Am J Hum Genet, 2007. 81(3): p. 559-75.

36. Benjamini, Y. and Y. Hochberg, Controlling the false discovery rate:a practical and powerful approach to multiple testing. J R Stat Soc Ser B Methodol, 1995. 57 p. 289-300.

37. Murcray, C.E., J.P. Lewinger, and W.J. Gauderman, Gene-environment interaction in genome-wide association studies. Am J Epidemiol, 2009. 169(2): p. 219-26.

38. Gao, X., J. Starmer, and E.R. Martin, A multiple testing correction method for genetic association studies using correlated single nucleotide polymorphisms. Genet Epidemiol, 2008. 32(4): p. 361-9.

39. Hein, D.W., et al., Molecular genetics and epidemiology of the NAT1 and NAT2 acetylation polymorphisms. Cancer Epidemiol Biomarkers Prev, 2000. 9(1): p. 29-42.

40. Cascorbi, I., et al., Arylamine N-acetyltransferase activity in man. Drug Metab Rev, 1999. 31(2): p. 489502.

41. Tiemersma, E.W., et al., Risk of colorectal adenomas in relation to meat consumption, meat preparation, and genetic susceptibility in a Dutch population. Cancer Causes Control, 2004. 15(3): p. 225-36.

42. Butler, L.M., et al., Modification by N-acetyltransferase 1 genotype on the association between dietary heterocyclic amines and colon cancer in a multiethnic study. Mutat Res, 2008. 638(1-2): p. 162-74.

43. Cantwell, M., et al., Relative validity of a food frequency questionnaire with a meat-cooking and heterocyclic amine module. Cancer Epidemiol Biomarkers Prev, 2004. 13(2): p. 293-8.

44. Felton, J.S. and M.G. Knize, Occurrence, identification, and bacterial mutagenicity of heterocyclic amines in cooked food. Mutat Res, 1991. 259(3-4): p. 205-17.

45. Malfatti, M.A. and J.S. Felton, Human UDP-glucuronosyltransferase $1 \mathrm{~A} 1$ is the primary enzyme responsible for the $\mathrm{N}$-glucuronidation of $\mathrm{N}$-hydroxy-PhIP in vitro. Chem Res Toxicol, 2004. 17(8): $\mathrm{p}$. 1137-44.

46. Girard, H., et al., UGT1A1 polymorphisms are important determinants of dietary carcinogen detoxification in the liver. Hepatology, 2005. 42(2): p. 448-57.

47. Girard, H., et al., UGT1A1 and UGT1A9 functional variants, meat intake, and colon cancer, among Caucasians and African-Americans. Mutat Res, 2008. 644(1-2): p. 56-63.

48. Butler, L.M., et al., Joint effects between UDP-glucuronosyltransferase $1 \mathrm{~A} 7$ genotype and dietary carcinogen exposure on risk of colon cancer. Cancer Epidemiol Biomarkers Prev, 2005. 14(7): p. 162632.

49. Polimanti, R., S. Piacentini, and M. Fuciarelli, HapMap-based study of human soluble glutathione Stransferase enzymes: the role of natural selection in shaping the single nucleotide polymorphism diversity of xenobiotic-metabolizing genes. Pharmacogenet Genomics, 2011. 21(10): p. 665-72. 
50. Felton, J.S., et al., Effect of microwave pretreatment on heterocyclic aromatic amine mutagens/carcinogens in fried beef patties. Food Chem Toxicol, 1994. 32(10): p. 897-903.

51. Salmon, C.P., M.G. Knize, and J.S. Felton, Effects of marinating on heterocyclic amine carcinogen formation in grilled chicken. Food Chem Toxicol, 1997. 35(5): p. 433-41.

52. Tran, N.L., et al., Experimental and simulation studies of heat flow and heterocyclic amine mutagen/carcinogen formation in pan-fried meat patties. Food Chem Toxicol, 2002. 40(5): p. 673-84.

53. Griesenbeck, J.S., et al., Development of estimates of dietary nitrates, nitrites, and nitrosamines for use with the Short Willet Food Frequency Questionnaire. Nutr J, 2009. 8: p. 16. 

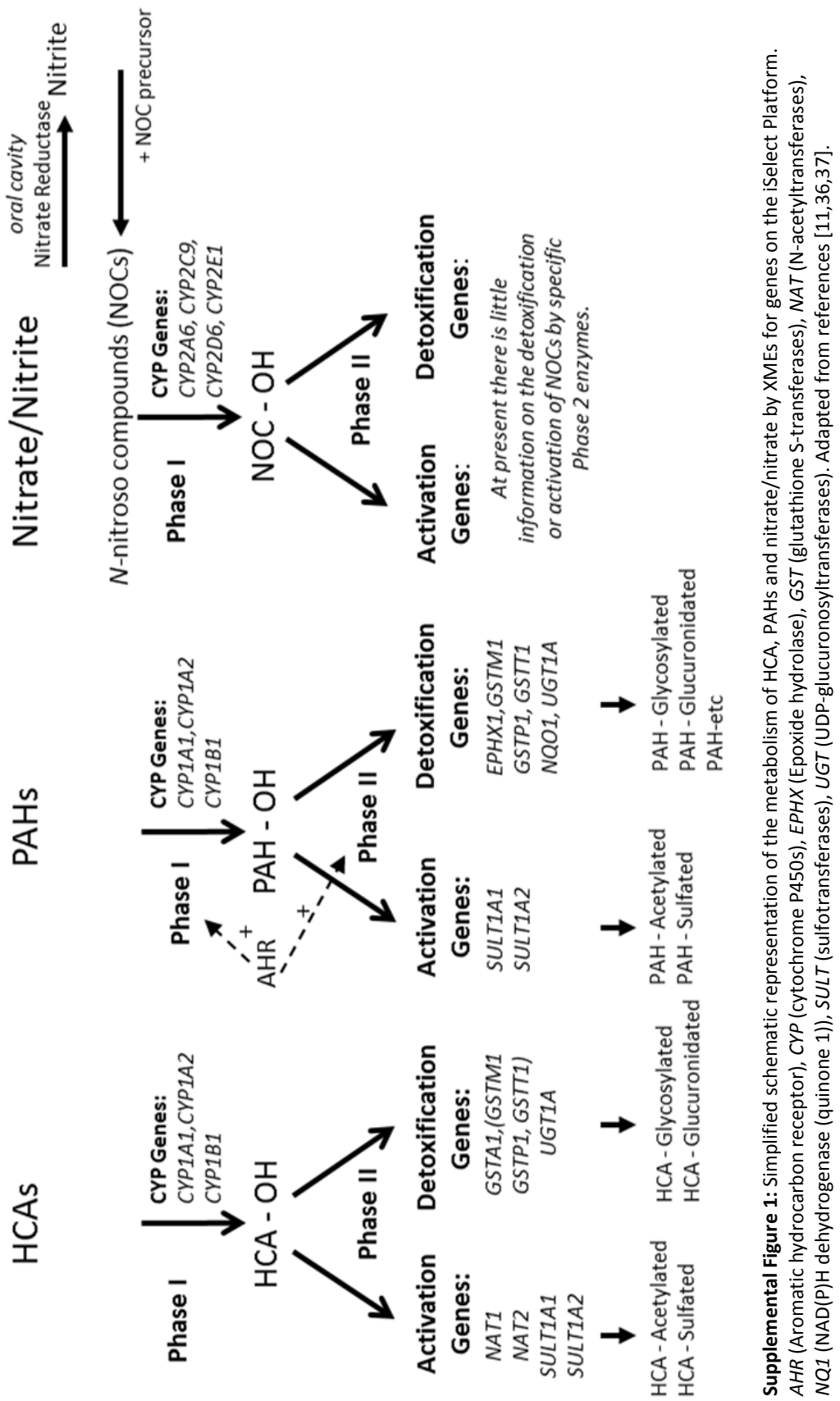


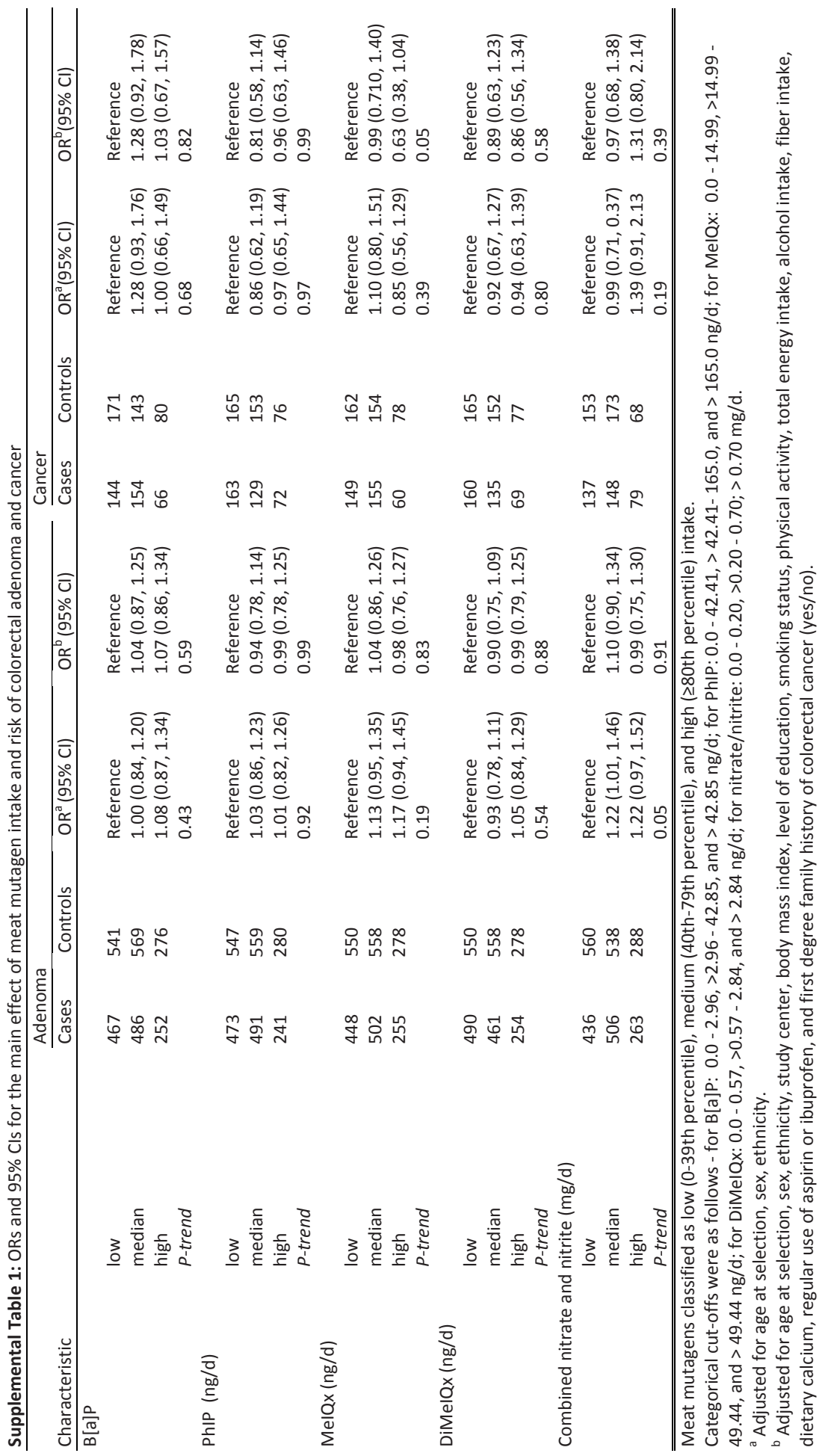


Supplemental Table 2: OR and 95\% $\mathrm{Cl}$ for the main effect of SNPs in xenobiotic metabolizing enzyme genes with $P<0.05$ on colorectal adenoma and cancer in the PLCO Cancer Screening Trial.

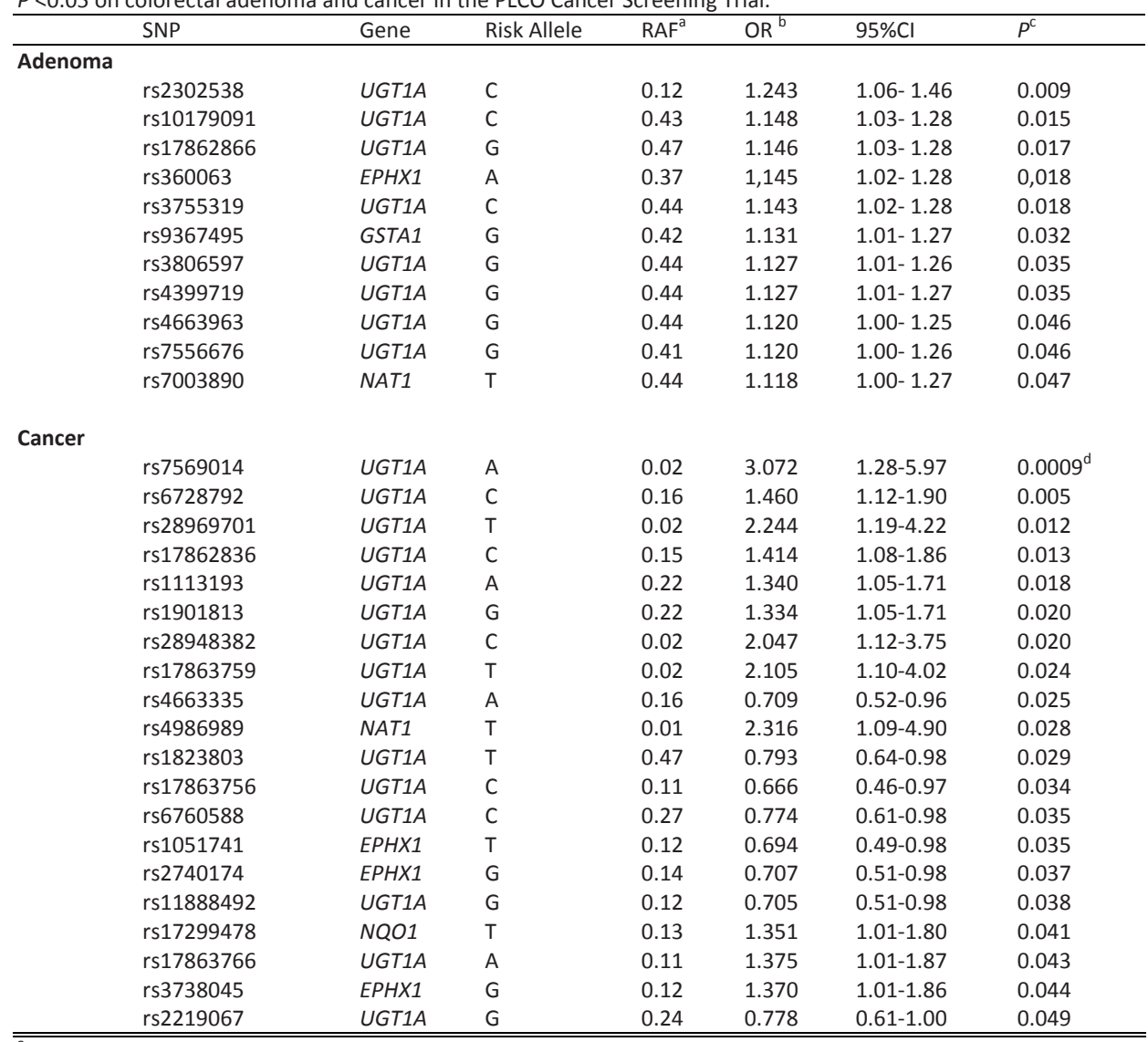

\footnotetext{
${ }^{a}$ RAF, risk allele frequency among Caucasian controls.

${ }^{\mathrm{b}}$ OR per risk allele assuming a log-additive model, adjusted for age, sex and ethnicity.

${ }^{\mathrm{c}}$ No associations were significant after adjusting for multiple testing using the False Discovery Rate.

${ }^{\mathrm{d}}$ Significant after gene-based correction.
} 


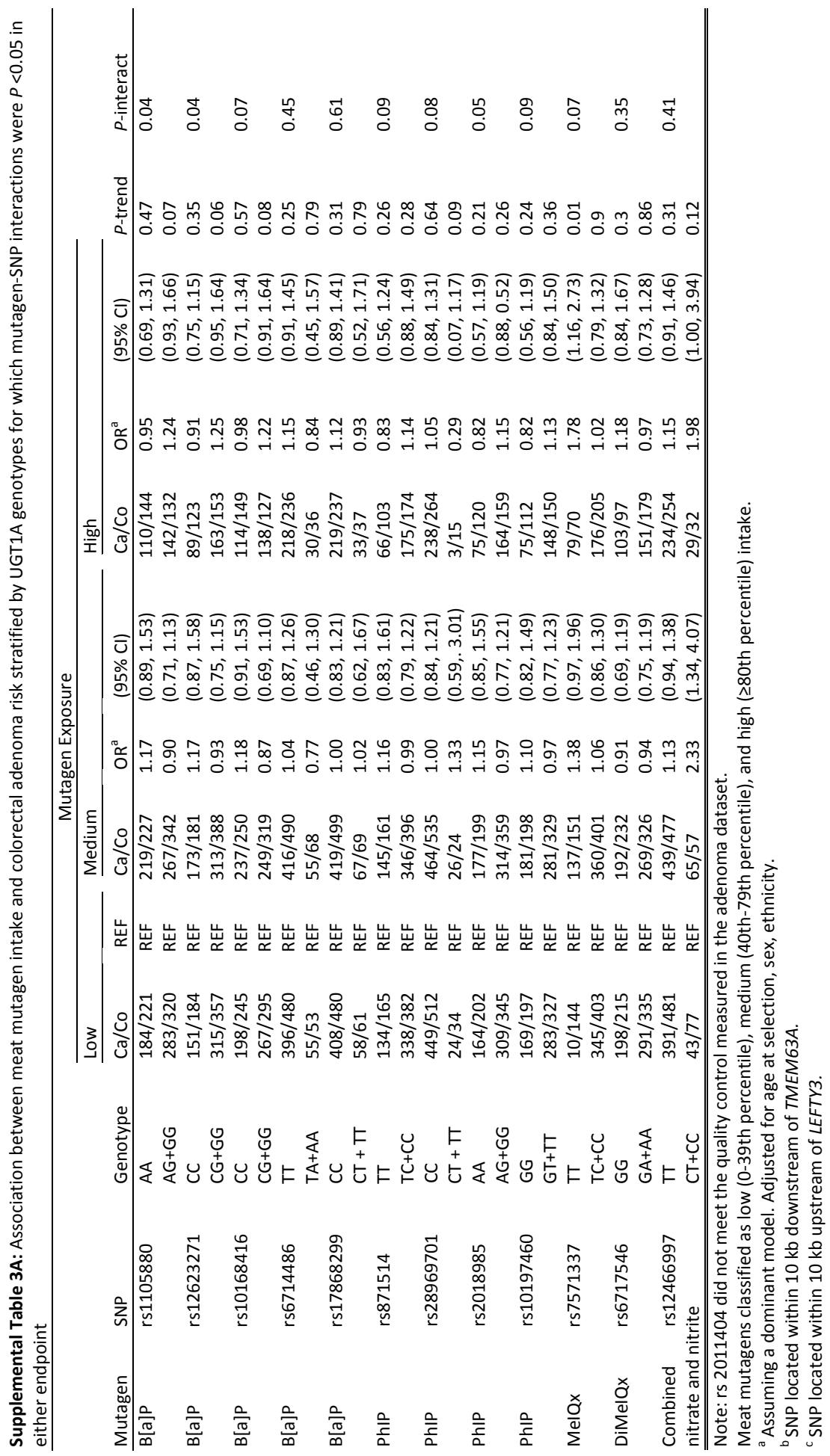




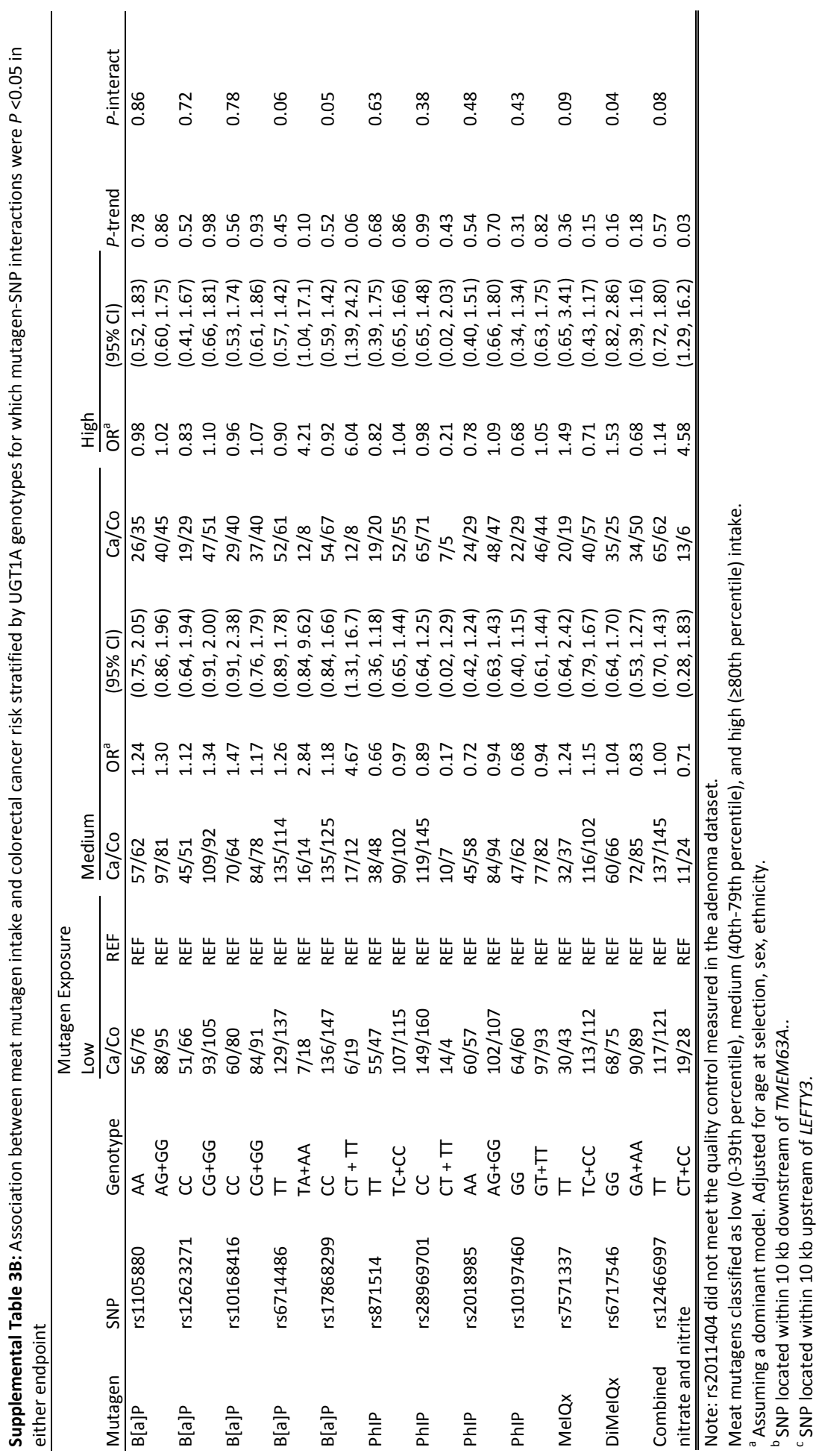


Supplemental Table 4: OR and 95\% $\mathrm{Cl}$ for the main effect of SNPs in xenobiotic metabolizing enzyme genes that interact with the meat mutagens $P<0.10$ on colorectal adenoma and cancer in the PLCO Cancer Screening Trial.

\begin{tabular}{|c|c|c|c|c|c|c|c|}
\hline & SNP & Gene & $\begin{array}{l}\text { Risk } \\
\text { Allele }\end{array}$ & $\mathrm{RAF}^{\mathrm{a}}$ & $O R^{b}$ & $95 \% \mathrm{Cl}$ & $P^{c}$ \\
\hline \multicolumn{8}{|l|}{ Adenoma } \\
\hline & rs15864 & GSTM3 (downstream) ${ }^{d}$ & C & 0.31 & 0,98 & $0.87-1.11$ & 0.75 \\
\hline & rs1105880 & UGT1A locus & G & 0.35 & 1.00 & $0.89-1.13$ & 0.96 \\
\hline & rs12623271 & UGT1A locus & G & 0.41 & 1.05 & $0.93-1.17$ & 0.44 \\
\hline & rs10168416 & UGT1A locus & G & 0.33 & 1.01 & $0.90-1.14$ & 0.83 \\
\hline & rs871514 & UGT1A locus & C & 0.44 & 1,12 & $0.99-1.25$ & 0.06 \\
\hline & rs28969701 & UGT1A locus & $\mathrm{T}$ & 0.02 & 0.85 & $0.59-1.23$ & 0.40 \\
\hline & rs2018985 & UGT1A locus & G & 0.38 & 1.11 & $0.99-1.25$ & 0.06 \\
\hline & rs10197460 & UGT1A locus & $\mathrm{T}$ & 0.39 & 1.05 & $0.94-1.18$ & 0.37 \\
\hline & rs4236290 & $A H R$ & C & 0.12 & 1.04 & $0.88-1.23$ & 0.63 \\
\hline & rs2671272 & $E P H X 1$ (downstream) ${ }^{\mathrm{e}}$ & $A$ & 0.21 & 1.07 & $0.93-1.22$ & 0.34 \\
\hline & rs7571337 & UGT1A locus & $\mathrm{T}$ & 0.51 & 1.03 & $0.92-1.15$ & 0.62 \\
\hline & rs6586714 & NAT1 & $A$ & 0.12 & 0.96 & $0.81-1.14$ & 0.64 \\
\hline & rs9332197 & CYP2C9 & C & 0.05 & 1.10 & $0.85-1.43$ & 0.47 \\
\hline & rs868966 & $E P H X 1$ (upstream) $^{f}$ & G & 0.44 & 1,07 & $0.95-1.19$ & 0.27 \\
\hline \multicolumn{8}{|l|}{ Cancer } \\
\hline & rs2066853 & $A H R$ & $A$ & 0.10 & 0.81 & $0.59-1.12$ & 0.21 \\
\hline & rs6714486 & UGT1A locus & A & 0.06 & 0.85 & $0.53-1.37$ & 0.50 \\
\hline & rs17868299 & UGT1A locus & $\mathrm{T}$ & 0.05 & 0.90 & $0.56-1.45$ & 0.67 \\
\hline & rs2011404 & UGT1A locus & $\mathrm{T}$ & 0.14 & 0.84 & $0.60-1.17$ & 0.30 \\
\hline & rs915908 & CYP2E1 & $A$ & 0.15 & 0,93 & $0.69-1.25$ & 0.63 \\
\hline & rs6717546 & UGT1A locus & A & 0.35 & 0.93 & $0.76-1.15$ & 0.52 \\
\hline & rs12466997 & UGT1A locus & C & 0.06 & 0.75 & $0.49-1.13$ & 0.17 \\
\hline
\end{tabular}

\footnotetext{
${ }^{\mathrm{a}} \mathrm{RAF}$, risk allele frequency among Caucasian controls.

${ }^{\mathrm{b}}$ OR per risk allele assuming a log-additive model, adjusted for age, sex and ethnicity.

${ }^{c}$ No associations were significant after adjusting for multiple testing using the False Discovery Rate.

${ }^{\mathrm{d}}$ SNP located within $10 \mathrm{~kb}$ downstream of EPS8L3.

e SNP located within $10 \mathrm{~kb}$ downstream of TMEM63A.

${ }^{f}$ SNP located within $10 \mathrm{~kb}$ upstream of LEFTY3.
} 


\section{CHAPTER 9}

\section{Dietary heme iron and the risk of colorectal cancer with specific mutations in KRAS and APC}

Anne M.J. Gilsing

Fiona Fransen

Theo M. de Kok

Alexandra R. Goldbohm

Leo J. Schouten

Adriaan P. de Bruïne

Manon van Engeland

Piet A. van den Brandt

Anton F.P.M. de Goeij and Matty P. Weijenberg

Carcinogenesis 2013;34(12):2757-66 


\section{ABSTRACT}

Background: Red meat intake has been linked to increased colorectal cancer (CRC) risk. Although the underlying mechanisms remain unclear, experimental studies suggest a role for dietary heme iron. Because heme iron was shown to promote specific mutations, it would be insightful to link heme iron data to CRC with mutations in key-genes in an observational, population-based study.

Methods: We investigated the association between dietary heme iron intake and risk of CRC with mutations in APC (adenomatous polyposis coli) and KRAS (Kirsten ras), and P53 overexpression in the Netherlands Cohort Study. After 7.3 y of follow-up, excluding the first 2.3 y due to incomplete coverage of the pathology registry and to avoid pre-clinical disease, adjusted hazard ratios (HR) (including adjustment for total meat) and $95 \%$ confidence intervals (Cl) were calculated, using 4026 subcohort members (aged 55-69 y at baseline), 435 colon and 140 rectal cancer patients.

Results: When comparing the highest with the lowest tertile of intake, heme iron intake was associated with an increased risk of CRC harboring activating mutations in KRAS ( $\mathrm{HR}=1.71,95 \% \mathrm{Cl}: 1.15-2.57$; P-trend=0.03) and $\mathrm{CRC}$ without truncating mutations in APC ( $\mathrm{HR}=1.79,95 \% \mathrm{Cl}: 1.23-2.60$; P-trend=0.003). We observed a positive association between heme iron intake and the risk of CRC with activating $\mathrm{G}>\mathrm{A}$ mutations in KRAS (P-trend=0.01), and overall $\mathrm{G}>\mathrm{A}$ mutations in $A P C$ ( $P$-trend=0.005). No associations were found with $\mathrm{CRC}$ harboring G>T mutations in KRAS/APC. Heme iron intake was positively associated with the risk of P53 overexpressed tumors, but not with tumors without P53 overexpression $(P$ heterogeneity $=0.12$ ).

Conclusion: Heme iron intake was associated with an increased risk of colorectal tumors harboring $\mathrm{G}>\mathrm{A}$ transitions in KRAS and $A P C$ and overexpression of $\mathrm{P} 53$. These novel findings suggest that alkylating rather than oxidative DNA damaging mechanisms are involved in heme-induced colorectal carcinogenesis. 


\section{INTRODUCTION}

Although the accumulated evidence from prospective epidemiological studies supports a positive association between the consumption of red meat and colorectal cancer risk [1-3], very few human studies have comprehensively evaluated the potential underlying mechanisms.

Experimental studies suggest a role for dietary heme iron, which is present at five-fold higher concentrations in red than white meat [4] and could explain why white meat, in contrast to red meat, is not associated with cancer risk [3]. Nevertheless, epidemiological evidence for its carcinogenic potential is inconclusive [5-10]. A recently conducted metaanalysis of five prospective studies showed a consistent, though modest, increased risk of $18 \%$ comparing the highest to the lowest category of heme iron intake [11].

Colorectal cancer is a heterogeneous endpoint, and the association between heme iron intake and colorectal cancer may become more evident when the underlying molecular events are taken into account. Most sporadic colorectal cancers are thought to arise through genetic pathways involving a stepwise pattern of mutations in the adenomatous polyposis coli $(A P C)$ and tumor protein 53 (TP53) tumor suppressor genes and the Kirsten ras (KRAS) oncogene [12]. These key genes are frequently altered in colorectal cancer [12]. Interestingly, experimental evidence suggests that heme iron and its metabolic products may increase the overall mutation rate and promote specific point mutations in the DNA of colonic tissue. For example, heme was shown to catalyze the endogenous formation of $\mathrm{N}$ nitroso compounds (NOC) [13], some of which are direct alkylating agents, whereas others need to be metabolized before yielding DNA alkylating intermediates (18). Such metabolites may lead to the induction of nitroso-compound-specific DNA adducts, such as 0-6-methylguanine [14], and have shown to induce G>A transitions in a variety of genes including KRAS and TP53 in both rodent and in vitro studies [15-17]. Furthermore, both heme iron and NOC may also catalyze the formation of reactive oxygen species that can cause DNA damage [18, 19], which, if not repaired, may induce $G>T$ transversions [20]. Lastly, heme is thought to promote colonic cytotoxicity and increased cell proliferation resulting in an increased overall mutation rate [21-23].

Linking heme iron exposure data to colorectal cancer with mutations in the colorectal cancer key genes APC, TP53 and KRAS in an observational, population-based study may provide new insight in the involvement of these genes in meat associated colorectal cancer risk. Although phenotypic expression studies in normal colonic tissue suggest that environmental factors can indeed influence protein expression patterns of these genes [24], previous studies linking meat consumption to (specific) mutations have yielded mixed results [25-29]. However, we are the first to hypothesize that heme iron and/or its metabolites, contribute to the observed colorectal cancer heterogeneity by increasing both the overall mutation rate and the occurrence of specific $\mathrm{G}>\mathrm{A}$ and $\mathrm{G}>\mathrm{T}$ genetic alterations in the $A P C$ and KRAS gene in colon and rectal tumors, that are characteristic for past heme iron exposure. Because mutation status of the TP53 gene was unavailable for the current analysis, we examined P53 overexpression status in relation to heme iron intake, a phenotype which has previously been inconsistently linked to meat intake [30, 31]. We further examined whether these associations differ by chlorophyll and dietary calcium 
intake because these may block the reactivity of heme iron in the gastrointestinal tract and thus prevent heme-induced colorectal carcinogenesis [32, 33].

\section{METHODS}

The Netherlands Cohort Study was initiated in September 1986 and includes 120852 men and women aged 55-69 years at baseline, originating from 204 municipalities with computerized population registries. Full details of the study design have been described elsewhere [34]. At the start of the study, participants completed a self-administered questionnaire on dietary habits, lifestyle characteristics, medical history, and other potential risk factors for cancer. The case-cohort approach was used for reasons of efficiency in questionnaire processing and follow-up. Case subjects were enumerated from the entire cohort, whereas the accumulated person years of the entire cohort were estimated from a random subcohort of 5000 men and women, chosen immediately after baseline. The entire cohort is being monitored for cancer occurrence by annual record linkage to the Netherlands Cancer Registry (NCR) and the Netherlands Pathology Registry (PALGA), together providing an almost complete coverage [35].

\section{Dietary exposure assessment}

All participants completed a 150 item semi-quantitative food-frequency questionnaire (FFQ) at baseline, estimating the average frequency and amounts of foods and beverages consumed over the previous 12 months. Daily mean nutrient intakes were calculated from the FFQ dietary data by summing the multiplied frequencies and number of serving sizes of all food items with their tabulated nutrient contents from the Dutch food composition table [36].

The FFQ contained 14 items on consumption of meat with the hot meal, 5 items on consumption of processed meat used as sandwich fillings, and 3 items on fish. The heme iron content from these meat items as well as the meat used in mixed dishes was estimated as an animal-specific percentage of total iron, derived from data in the literature (e.g. 65, 39 , and 26 percent for cooked beef, pork and chicken or fish, respectively), and has been reported in detail elsewhere [7].

The FFQ has been validated and tested for reproducibility [37, 38]. Crude (energy and gender adjusted between brackets) Pearson correlation coefficients ${ }^{\circledR}$ between the questionnaire and the 9-day diet record (kept over 3-day periods, 4-5 months apart) were 0.74 for energy, $0.72(0.52)$ for total fat, $0.73(0.58)$ for saturated fatty acids (SFA) and 0.73 (0.75) for polyunsaturated fatty acids (PUFA). The Spearman correlation coefficients for fresh meat, processed meat, and fish were $0.46,0.54$ and 0.53 respectively [37].

\section{Tissue samples and DNA isolation}

Tumor material from incident colorectal cancer patients identified during 7.3 years of follow-up was collected after approval by the Ethical Review Board of Maastricht University, PALGA, and the NCR. The first 2.3 years of the follow-up period were excluded due to the incomplete coverage of PALGA and to avoid possible pre-clinical disease affecting exposure status. The tissue samples were distributed among 54 pathology 
laboratories throughout The Netherlands. Ninety percent (733 out of 815) of the available tissue samples contained sufficient tumor material for molecular analyses [39].

\section{$A P C$ and KRAS mutation analysis}

Mutation analysis of the mutation cluster region in the $A P C$ tumor suppressor gene (codons 1286-1520), and the exon 1 fragment of the KRAS oncogene (codons 12-13) were performed using a nested PCR approach, followed by direct sequencing of purified fragments. This procedure has been described in details elsewhere [39, 40]. KRAS status was available for all, and APC status was available for $90 \%$ of the cases.

\section{TP53 expression analysis}

Immunohistochemical staining for P53 was performed according to the avidin - biotin peroxidase complex method, using the DO-7 mouse monoclonal antibody (DAKO A/S, Denmark) as previously described [41]. Immunostained slides and negative controls were evaluated semi-quantitatively and independently by two observers without knowledge of clinical parameters. We defined cases positive for overexpression of TP53 if $20 \%$ or more of the tumor cell nuclei showed positive staining with the antibody[41]. For $99 \%$ of the cases, P53 expression data was available.

\section{Statistical analysis}

Colorectal cancer was classified according to site: colon (ICD-O codes: 153.0- 153.7); rectosigmoid (ICD-O code 154.0) and rectum (ICD-O code 154.1). Rectosigmoid cancer cases were not evaluated separately because of the small number of cases $(n=69)$ and the higher risk of misclassification [42]. After 7.3 y of follow-up and exclusion of prevalent cancer cases at baseline (other than skin cancer $(n=226)$, subjects with an incomplete food frequency questionnaires $(n=381)$, or subjects with missing information on confounders $(n=349), 4026$ subcohort members and 644 colorectal (435 colon and 140 rectum cancer cases) remained eligible for analyses.

All analyses were conducted separately for colorectal, colon or rectum tumors with or without a truncating mutation in $A P C$, an activating mutation in KRAS and overexpression of P53. Truncating APC mutations lead to the introduction of a stopcodon and result in a truncated and therefore inactive APC protein. Activating KRAS mutations are defined as mutations in codons 12 and 13 of the KRAS gene leading to an altered amino acid resulting in an activated RAS protein.

We additionally classified the colorectal, colon and rectum tumors according to specific point mutations ( $\mathrm{G}>\mathrm{A}$ and $\mathrm{G}>\mathrm{T}$ ) in APC and KRAS according to two different methods, either focusing on overall mutations or restricting to functional (activating/truncating) mutations. Moreover, additional analyses were performed combining tumors with specific genetic aberrations in the APC and KRAS gene (e.g. tumors with no $\mathrm{G}>\mathrm{A}$ mutation in both KRAS and $A P C)$.

Hazard ratios (HR) and corresponding 95\% confidence intervals $(\mathrm{Cl})$ were estimated using the multivariate Cox proportional hazards model. The total person-years at risk, estimated from the subcohort, were used in analyses [43]. Standard errors were estimated using the robust Huber-White sandwich estimator to account for additional variance introduced by 
sampling from the cohort [44]. The proportional hazards assumption was tested using the scaled Schoenfeld residuals.

Tests for heterogeneity were performed to evaluate differences in the association with heme iron between tumors with (specific) aberrations and tumors without any aberrations, using the competing risks procedure in STATA. However, the standard error for the difference of the log HRs from this procedure assumes independence of both estimated HRs, which would overestimate this standard error and thus overestimate the $p$-values for their difference; therefore, parameter estimates were based on a bootstrapping method that was developed for the case-cohort design [45]. For each bootstrap sample, $X$ subcohort members were randomly drawn from the subcohort of $X$ subjects and $Y$ cases from the total of $Y$ cases outside the subcohort, both with replacement, out of the dataset of $X+Y$ observations. The log HRs were obtained from this sample using the competing risks procedure and recalculated for each bootstrap replication. The $\mathrm{Cl}$ and $\mathrm{p}$-value of the differences in hazard ratio for the molecular subtypes were then calculated from the replicated statistics. Each bootstrap analysis was based on 1000 replications [46].

The covariates included in the multivariate analyses were either a priori selected risk factors of colorectal cancer or variables that changed one of the risk estimates by $10 \%$ or more. This resulted in a multivariable model including age at baseline (y), sex, intake of alcohol (0, 0.1-29.9, >=30 g/day), vegetable consumption (g/day), total energy intake (kcal/day), BMI $\left(\mathrm{kg} / \mathrm{m}^{2}\right)$, family history of colorectal cancer (yes, no), smoking status (never, former, current), non-occupational physical activity $(<30,30-60,60-90,>90 \mathrm{~min} / \mathrm{d})$. In addition, we calculated a third model in which we additionally adjusted for the intake of total fresh meat (g/day) and processed meat (g/day), to assess the effect of heme iron independent of meat intake.

Subjects were classified into tertiles of heme iron consumption based on the subcohort (with the lowest tertile regarded as the reference group), and as a continuous variable (1 $\mathrm{mg} /$ day increment). Heme iron intake was adjusted for total energy intake using the residual regression method [47]. For each analysis, trends were evaluated with the Wald test by assigning participants the median value for each level of the categorical exposure variables among the subcohort members and this variable was entered as a continuous term in the Cox regression model.

Possible effect modification by sex, chlorophyll, dietary calcium, BMI, smoking status, and physical activity were tested by entering cross-product terms in the model. However, none of these interaction terms was statistically significant $(P$-interaction $>0.01)$.

All analyses were performed using STATA Statistical Software (Intercooled STATA, version 10; Stata-Corp LP, College Station, TX). All tests were two-tailed and differences were regarded as statistically significant at $P<0.05$.

\section{RESULTS}

The baseline characteristics of subcohort members, colorectal, colon and rectum cancer cases, of which the greater part did not differ significantly between the groups, are shown in Table 1. Colorectal, colon and rectum cancer patients were older, more often men, had a 
higher percentage of former smokers and individuals with a family history of colorectal cancer as compared to the subcohort.

Table 1: Baseline characteristics and dietary intakes of exposures of interest of colorectal cancer cases and subcohort members within the Netherlands Cohort Study

\begin{tabular}{|c|c|c|c|c|}
\hline Characteristic & Subcohort & $\begin{array}{l}\text { Colorectal cancer } \\
\text { cases }\end{array}$ & $\begin{array}{l}\text { Colon cancer } \\
\text { cases }\end{array}$ & $\begin{array}{l}\text { Rectum cancer } \\
\text { cases }\end{array}$ \\
\hline $\mathrm{N}$ & 4026 & 644 & 435 & 140 \\
\hline Age (y) & $61.3 \pm 4.2^{a}$ & $62.8 \pm 4.1$ & $62.9 \pm 4.1$ & $62.3 \pm 4.0$ \\
\hline Sex (\% male) & 49.8 & 56.5 & 54.1 & 65.7 \\
\hline \multicolumn{5}{|l|}{ Dietary factors } \\
\hline Total heme iron intake (mg/day) & $1.1 \pm 0.5$ & $1.1 \pm 0.5$ & $1.1 \pm 0.5$ & $1.1 \pm 0.4$ \\
\hline Total fresh meat intake (g/day) ${ }^{\mathrm{b}}$ & $99.5 \pm 41.7$ & $99.0 \pm 37.5$ & $99.5 \pm 36.3$ & $96.2 \pm 38.4$ \\
\hline Total processed meat intake (g/day) & $14.3 \pm 15.6$ & $14.9 \pm 16.3$ & $14.8 \pm 15.9$ & $14.8 \pm 13.8$ \\
\hline Chlorophyll (mg/day) & $52.2 \pm 29.5$ & $52.3 \pm 27.4$ & $51.3 \pm 26.9$ & $52.1 \pm 27.4$ \\
\hline Vegetables (g/day) & $194.1 \pm 82.1$ & $193.6 \pm 85.0$ & $191.6 \pm 81.5$ & $193.1 \pm 92.0$ \\
\hline Energy intake (kJ/day) & $8072 \pm 2161$ & $8094 \pm 2018$ & $8057 \pm 2007$ & $8343 \pm 1891$ \\
\hline \multicolumn{5}{|l|}{ Alcohol (\%) } \\
\hline 0 (g/day) & 23.3 & 23.1 & 25.5 & 19.3 \\
\hline $0.1-29.9$ (g/day) & 67.6 & 64.6 & 62.8 & 65.7 \\
\hline$>=30$ (g/day) & 9.1 & 12.3 & 11.7 & 15.0 \\
\hline Family history of colorectal cancer (\% yes) & 5.7 & 11.0 & 12.0 & 10.7 \\
\hline Body mass index $\left(\mathrm{kg} / \mathrm{m}^{2}\right)$ & $25.0 \pm 3.1$ & $25.5 \pm 3.1$ & $25.5 \pm 3.2$ & $25.2 \pm 2.9$ \\
\hline \multicolumn{5}{|l|}{ Smoking status (\%) } \\
\hline Never & 35.3 & 32.3 & 35.6 & 29.3 \\
\hline Former & 36.4 & 45.3 & 44.1 & 45.0 \\
\hline Current & 28.4 & 22.4 & 20.2 & 25.7 \\
\hline \multicolumn{5}{|l|}{ Non-occupational physical activity (\%) } \\
\hline$<30 \mathrm{~min} /$ day & 20.4 & 20.4 & 20.7 & 22.1 \\
\hline 30-60 min/day & 31.6 & 31.1 & 32.0 & 27.9 \\
\hline $60-90 \mathrm{~min} / \mathrm{day}$ & 20.9 & 21.5 & 20.7 & 22.1 \\
\hline$>90 \mathrm{~min} / \mathrm{day}$ & 27.0 & 27.2 & 26.7 & 27.9 \\
\hline
\end{tabular}

${ }^{a}$ Mean \pm SD (all such values).

${ }^{b}$ Intake of fresh meat items were based on raw-meat weights.

No differences between colon and rectum cancer patients and subcohort members were observed regarding baseline heme iron intake in women (mean intake of 0.99, 0.99 and $0.97 \mathrm{mg} /$ day, respectively). In men, daily heme iron intake was highest in colon cancer cases $(1.23 \mathrm{mg})$, followed by the subcohort $(1.15 \mathrm{mg})$, and lowest in rectum cancer cases (1.10 mg) (results not shown). Also, no statistically significant differences in heme iron intake were observed between colon or rectum cancer cases characterized by specific molecular tumor characteristics in both men and women (results not shown). The correlation of heme iron intake and total fresh meat, processed meat, and fresh and processed meat combined, were $0.60,0.42$, and 0.68 respectively, for men and $0.59,0.45$, and 0.68 respectively, for women (results not shown). Among the colorectal cancer cases, $56 \%$ had overexpression of P53 in the cell nucleus, 33\% had an activating KRAS mutation, and $36 \%$ had a truncating $A P C$ mutation.

Age- and sex adjusted (model 1), multivariable adjusted (model 2) and multivariable and fresh- and processed meat intake adjusted (model 3) HRs and corresponding $95 \% \mathrm{Cl}$ for different molecular endpoints of colorectal, colon and rectum cancer according to tertiles of heme iron intake are presented in Table 2A (colorectal) and Table 2B (colon and rectum). 
Table 2A: Hazard ratios (HR) and 95\% confidence intervals ( $\mathrm{Cl}$ ) for colorectal cancer with different molecular characteristics according to tertiles (T) of heme iron intake in the NLCS (7.3 years of follow-up) ${ }^{\mathrm{a}}$

\begin{tabular}{|c|c|c|c|c|c|}
\hline \multirow[b]{2}{*}{ Molecular endpoint } & \multirow[b]{2}{*}{ PY } & \multicolumn{4}{|c|}{ Colorectal cancer } \\
\hline & & Cases & $\mathrm{HR}(95 \% \mathrm{Cl})^{\mathrm{b}}$ & $\mathrm{HR}(95 \% \mathrm{Cl})^{\mathrm{c}}$ & $\operatorname{HR}(95 \% \mathrm{Cl})^{\mathrm{d}}$ \\
\hline \multicolumn{6}{|c|}{$A P C:$ without introduction of stopcodon } \\
\hline $\mathrm{T} 1$ & 6496 & 98 & 1 & 1 & 1 \\
\hline T2 & 6484 & 133 & $1.35(1.02-1.77)$ & $1.31(0.99-1.73)$ & $1.49(1.09-2.05)$ \\
\hline T3 & 6480 & 137 & $1.41(1.08-1.85)$ & $1.39(1.05-1.84)$ & $1.79(1.23-2.60)$ \\
\hline$P$-trend & & & 0.02 & 0.03 & 0.003 \\
\hline Continuous $(1 \mathrm{mg} / \mathrm{d}$ i & & & $1.17(0.98-1.41)$ & $1.17(0.96-1.42)$ & $1.40(1.06-1.84)$ \\
\hline \multicolumn{6}{|c|}{$A P C$ : introduction of stopcodon } \\
\hline T1 & 6496 & 68 & 1 & 1 & 1 \\
\hline $\mathrm{T} 2$ & 6484 & 64 & $0.93(0.66-1.33)$ & $0.89(0.62-1.26)$ & $0.89(0.60-1.33)$ \\
\hline T3 & 6480 & 77 & $1.14(0.82-1.60)$ & $1.06(0.75-1.48)$ & $1.03(0.65-1.64)$ \\
\hline$P$-trend & & & 0.41 & 0.69 & 0.82 \\
\hline Continuous ( $1 \mathrm{mg} / \mathrm{d}$ i & & & $1.24(0.94-1.64)$ & $1.16(0.86-1.56)$ & $1.22(0.79-1.89)$ \\
\hline \multicolumn{5}{|c|}{$P$-heterogeneity : $A P C$ with stopcodon vs. $A P C$ without stopcodon } & 0.16 \\
\hline \multicolumn{6}{|l|}{ KRAS: wildtype } \\
\hline $\mathrm{T} 1$ & 6496 & 134 & 1 & 1 & 1 \\
\hline T2 & 6484 & 133 & $0.99(0.77-1.27)$ & $0.94(0.73 .1 .22)$ & $0.99(0.74-1.32)$ \\
\hline $\mathrm{T3}$ & 6480 & 159 & $1.20(0.94-1.53)$ & $1.16(0.90 .1 .49)$ & $1.25(0.89-1.76)$ \\
\hline$P$-trend & & & 0.13 & 0.2 & 0.16 \\
\hline Continuous $(1 \mathrm{mg} / \mathrm{d}$ i & & & $1.19(0.99-1.44)$ & $1.18(0.96-1.45)$ & $1.33(0.99-1.77)$ \\
\hline \multicolumn{6}{|c|}{ KRAS: activating mutation } \\
\hline T1 & 6496 & 55 & 1 & 1 & 1 \\
\hline $\mathrm{T} 2$ & 6484 & 85 & $1.53(1.08-2.17)$ & $1.49(1.04-2.12)$ & $1.71(1.15-2.57)$ \\
\hline $\mathrm{T} 3$ & 6480 & 74 & $1.36(0.95-1.95)$ & $1.30(0.90-1.87)$ & $1.73(1.08-2.77)$ \\
\hline$P$-trend & & & 0.12 & 0.21 & 0.03 \\
\hline Continuous $(1 \mathrm{mg} / \mathrm{d} \mathrm{i}$ & & & $1.16(0.90-1.48)$ & $1.10(0.85-1.42)$ & $1.35(0.93-1.94)$ \\
\hline$P$-heterogeneity : mu & wildtyp & e KRAS & & & 0.14 \\
\hline \multicolumn{6}{|c|}{ TP53: without overexpression } \\
\hline $\mathrm{T} 1$ & 6496 & 79 & 1 & 1 & 1 \\
\hline $\mathrm{T} 2$ & 6484 & 106 & $1.33(0.98-1.80)$ & $1.28(0.94-1.75)$ & $1.29(0.91-1.83)$ \\
\hline $\mathrm{T} 3$ & 6480 & 93 & $1.19(0.87-1.63)$ & $1.15(0.83-1.58)$ & $1.15(0.75-1.76)$ \\
\hline$P$-trend & & & 0.31 & 0.47 & 0.63 \\
\hline Continuous $(1 \mathrm{mg} / \mathrm{d}$ i & & & $1.06(0.85-1.32)$ & $1.03(0.81-1.30)$ & $(0.95(0.67-1.35)$ \\
\hline \multicolumn{6}{|c|}{ TP53: overexpression } \\
\hline $\mathrm{T} 1$ & 6496 & 108 & 1 & 1 & 1 \\
\hline $\mathrm{T} 2$ & 6484 & 109 & $1.00(0.76-1.32)$ & $0.97(0.73 .1 .28)$ & $1.10(0.80-1.50)$ \\
\hline Т3 & 6480 & 138 & $1.29(0.98-1.68)$ & $1.25(0.95-1.63)$ & $1.58(1.10-2.27)$ \\
\hline$P$-trend & & & 0.05 & 0.09 & 0.008 \\
\hline Continuous $(1 \mathrm{mg} / \mathrm{d}$ i & & & $1.27(1.04-1.56)$ & $1.25(1.01-1.55)$ & $1.66(1.25-2.21)$ \\
\hline \multicolumn{5}{|c|}{$P$-heterogeneity : TP53 with overexpression vs. TP53 without overexpression } & 0.12 \\
\hline
\end{tabular}

T (tertile of heme intake); PY (Person years at risk).

${ }^{a} \mathrm{HR}$, hazard ratio. T1 was the reference category. HRs were derived from Cox regression analyses.

${ }^{\mathrm{b}}$ Adjusted for age at baseline (y) and sex.

${ }^{\mathrm{c}}$ Adjusted for gender, age at baseline (y),BMI (kg/m2), family history of colorectal cancer (yes, no), smoking (never, former, current), non-occupational physical activity $(<30,30-60,60-90,>90 \mathrm{~min} / \mathrm{d})$, total energy intake (kcal/day), alcohol consumption $(0,0.1-29.9,>=30 \mathrm{~g} /$ day) and total vegetable consumption $(\mathrm{g} /$ day)

${ }^{\mathrm{d}}$ Adjusted for gender, age at baseline (y),BMI (kg/m2), family history of colorectal cancer (yes, no), smoking (never, former, current), non-occupational physical activity $(<30,30-60,60-90,>90 \mathrm{~min} / \mathrm{d})$, total energy intake (kJ), alcohol consumption (0, 0.1-29.9, >= $30 \mathrm{~g} /$ day), total vegetable consumption (g/day), total fresh meat intake $(\mathrm{g} /$ day) and total processed meat intake (g/day). 


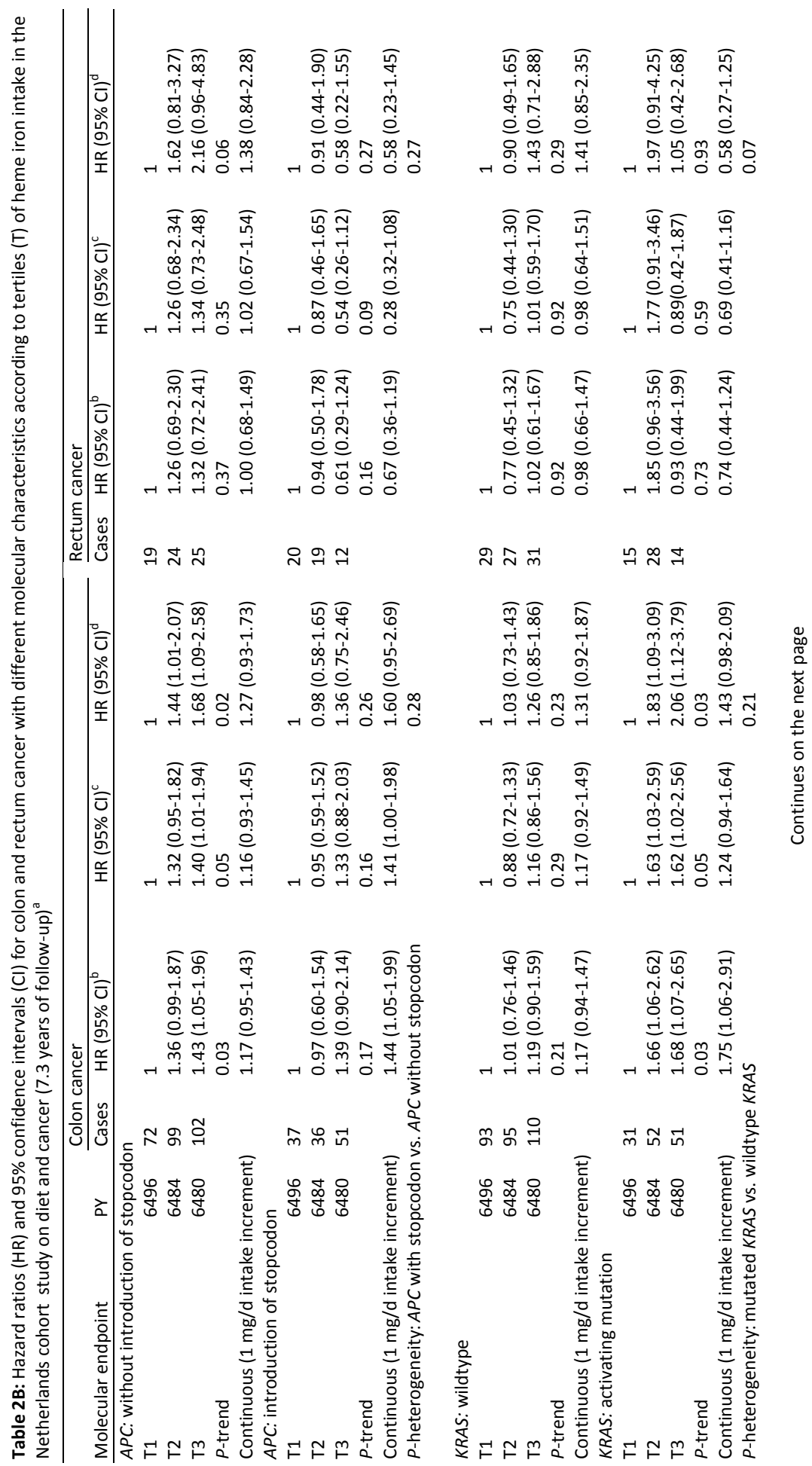




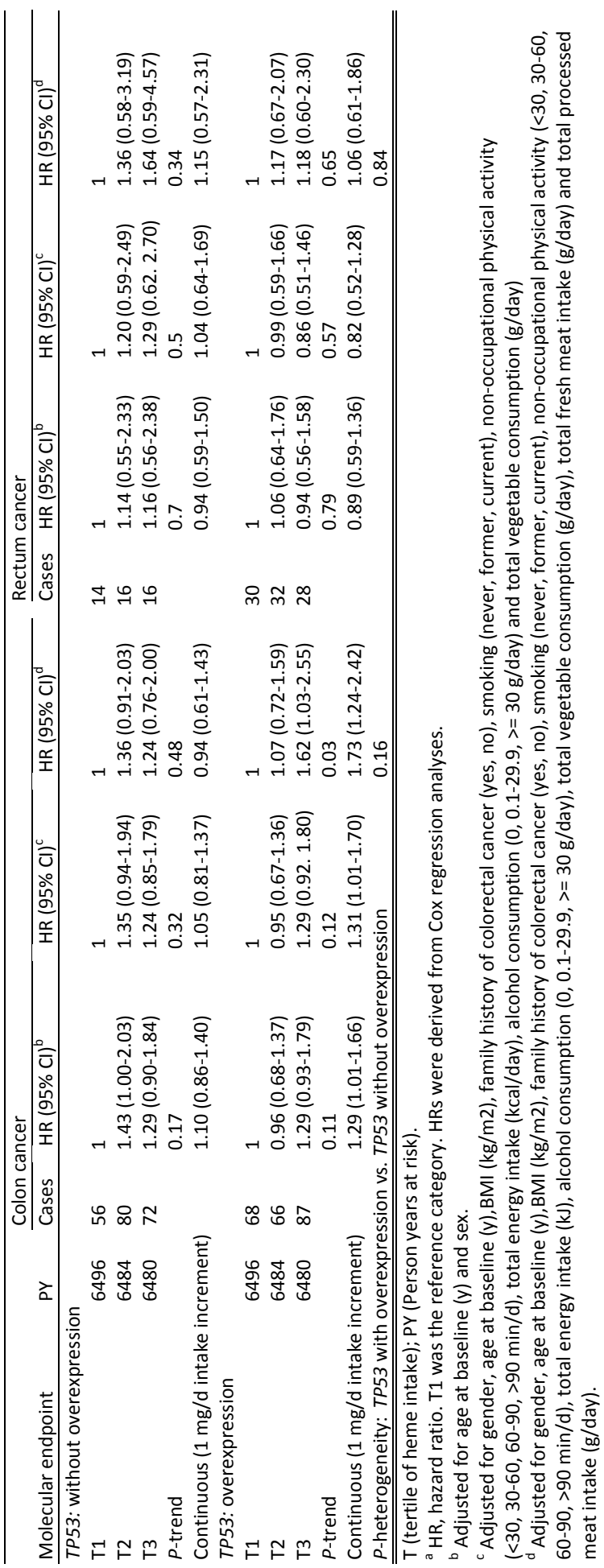


The HRs for heme iron and colorectal cancer were stronger when additionally adjusting the models for total fresh and processed meat. Heme iron intake was not associated with the risk of colorectal tumors harboring a stopcodon in APC. In contrast, a significantly increased $\mathrm{HR}$ between heme iron intake and the risk of colorectal tumors without mutations leading to the introduction of a stopcodon was observed (multivariable HR (model 3) highest versus lowest tertile: $1.79 ; 95 \% \mathrm{Cl}: 1.23,2.60 ; P$-trend=0.003; HR per $1 \mathrm{mg} /$ day increase: $1.40 ; 95 \%$ $\mathrm{Cl}$ : 1.06-1.84). Heme iron intake was significantly associated with colorectal tumors with activating KRAS gene mutations (multivariable HR (model 3) highest vs. lowest tertile: 1.73; 95\% Cl: 1.08-2.77; $P$-trend $=0.03$ ) and P53 overexpression in the cell nucleus (multivariable HR (model 3) for highest vs. lowest tertile: $1.58 ; 95 \% \mathrm{Cl}$ : 1.10-2.27; $P$-trend=0.008), but not with tumors without these aberrations. Comparable results were observed for colon cancer but not for rectum cancer (Table 2B).

Table 3A: Hazard ratios (HR) and 95\% confidence intervals (CI) for colorectal cancer with specific functional point mutations in colorectal key genes according to tertiles (T) of heme iron intake in the NLCS (7.3 years of follow-up) ${ }^{\text {a }}$

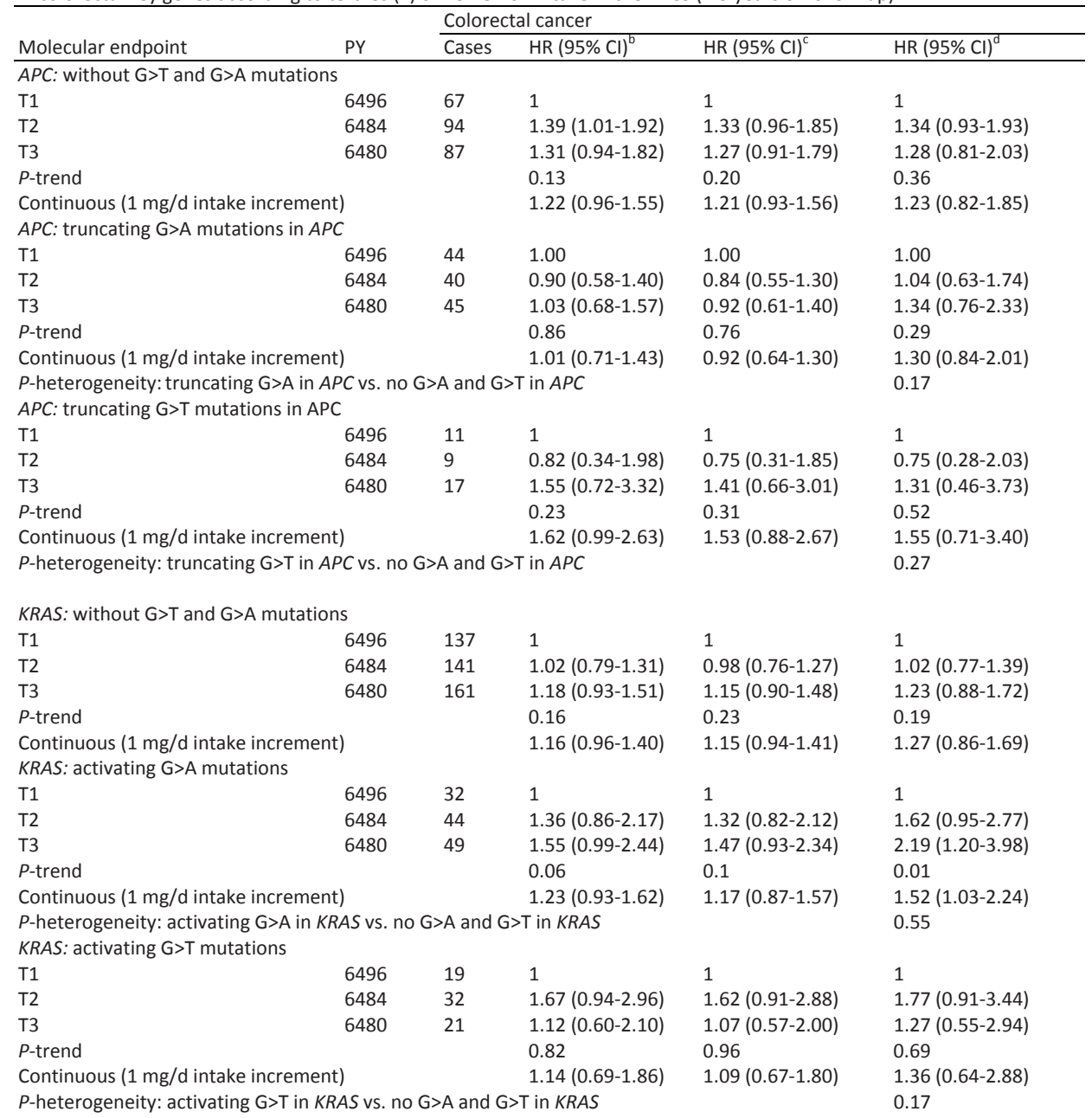




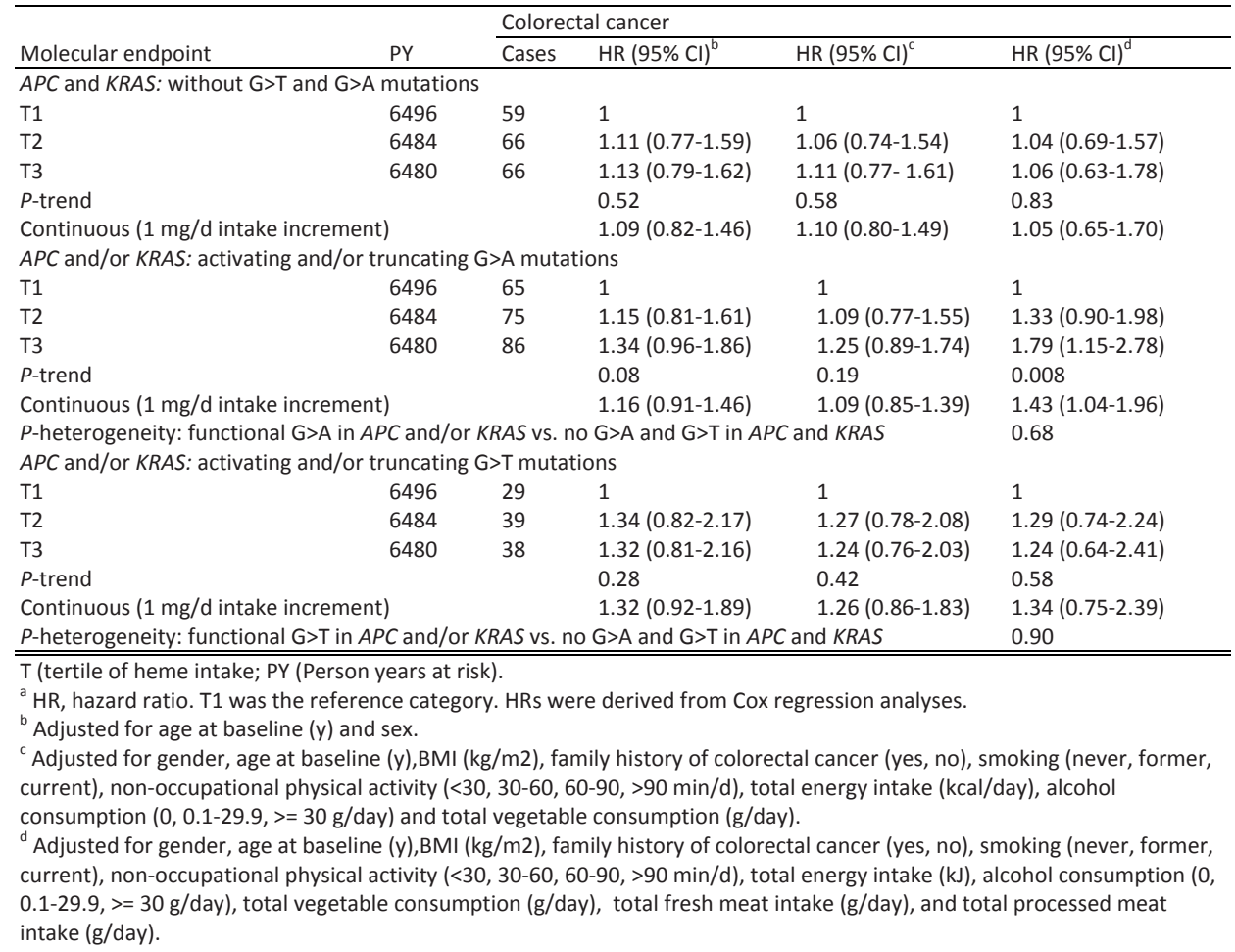

Additional analyses were performed to evaluate the association between heme iron intake and the risk of colorectal, colon and rectum tumors harboring specific functional (truncating/activating) point mutations ( $\mathrm{G}>\mathrm{A}$ transitions and/or $\mathrm{G}>\mathrm{T}$ transversions) in $A P C$ and KRAS (Table 3A (colorectal) and Table 3B (colon and rectum)). Again, associations were stronger after additional adjustment for total meat intake. No clear dose-response associations were observed between heme iron intake and the risk of colorectal, colon or rectum tumors harboring specific truncating mutations in $A P C$, nor for tumors without such $\mathrm{G}>\mathrm{A}$ and $\mathrm{G}>\mathrm{T}$ mutations.

In contrast, heme iron intake was associated with an increased risk of colorectal tumors harboring activating G>A KRAS mutations (multivariable HR (model 3) highest vs. lowest tertile of intake: 2.19; $95 \% \mathrm{Cl}: 1.20,3.98$; $P$-trend= 0.01 ; HR per $1 \mathrm{mg} /$ day increase: 1.52 ; $95 \% \mathrm{Cl}: 1.03-2.24)$. Such association was not found when focusing on activating $\mathrm{G}>\mathrm{T}$ mutations, or when looking at colorectal tumors without $\mathrm{G}>\mathrm{T}$ and $\mathrm{G}>\mathrm{A}$ mutations in KRAS. Similar associations were observed for colon but not for rectum cancer. In addition, heme iron intake was also associated with increased risk of colorectal tumors harboring activating/truncating $\mathrm{G}>\mathrm{A}$ mutations in either $A P C$ and/or KRAS (multivariable HR (model 3): 1.79; $95 \% \mathrm{Cl}$ : 1.15, 2.78; P-trend=0.008; HR per $1 \mathrm{mg} /$ day increase: 1.43 ; $95 \% \mathrm{Cl}$ : 1.04 1.96), but not with tumors harboring $\mathrm{G}>\mathrm{T}$ mutation, nor for tumors without such mutations in $A P C$ and $K R A S$ combined. 


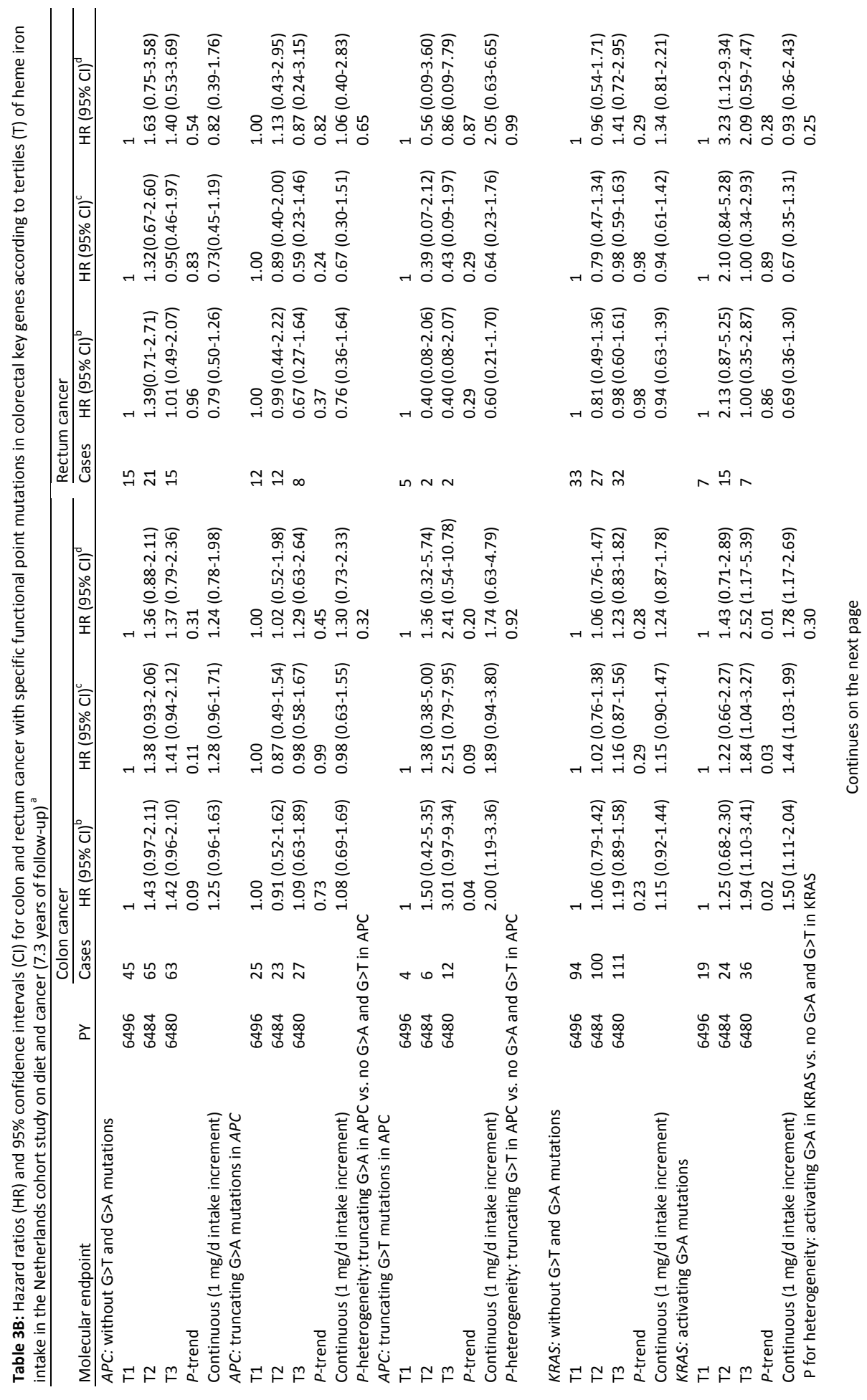




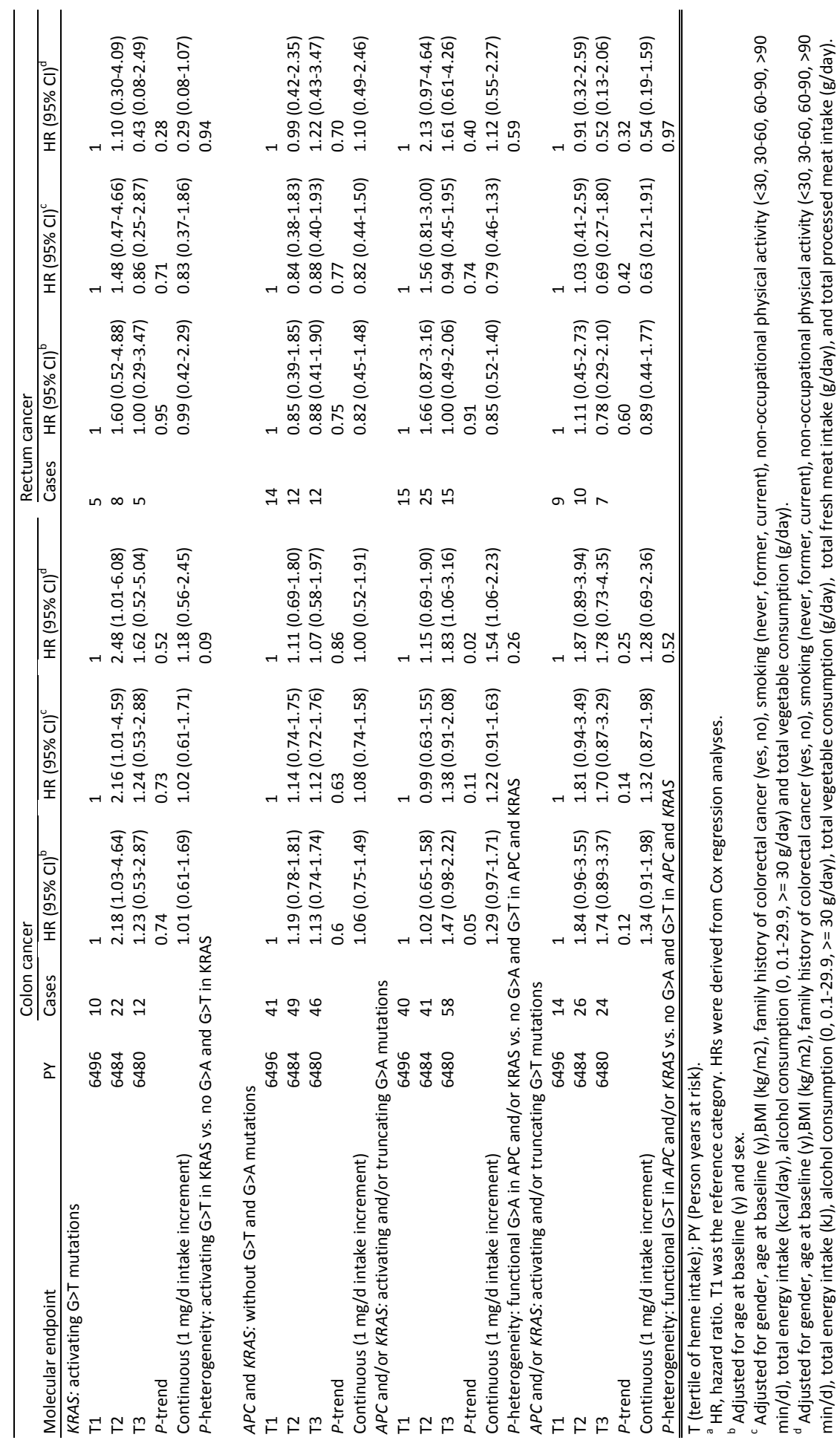


When classifying the colorectal tumors according to specific point mutations, while focusing on overall mutations instead of restricting to functional mutations, we generally found similar results (Table 4). In addition, heme iron intake was significantly associated with an increased risk of colorectal tumors harboring overall G $>A$ APC mutations (multivariable HR (model 3) highest vs. lowest tertile of intake: $1.71 ; 95 \% \mathrm{Cl}: 1.16,2.50 ; P$ trend=0.005; HR per $1 \mathrm{mg} /$ day increase: $1.43 ; 95 \% \mathrm{Cl}$ : 1.08-1.88).

None of the tests for heterogeneity comparing the association with heme iron between molecular subgroups (e.g. tumors with specific mutations versus tumors without the specific mutations) in any of the analyses presented herein were statistically significant (all $P$-heterogeneity $>0.05$ ).

Table 4: Hazard ratios (HR) and 95\% confidence intervals (CI) for colorectal cancer with specific overall point mutations in colorectal key genes according to tertiles $(T)$ of heme iron intake in the Netherlands cohort study on diet and cancer $\left(7.3\right.$ years of follow-up) ${ }^{\mathrm{a}}$

\begin{tabular}{|c|c|c|c|c|c|}
\hline \multirow[b]{2}{*}{ Molecular endpoint } & \multirow[b]{2}{*}{ PY } & \multicolumn{4}{|c|}{ Colorectal cancer } \\
\hline & & Cases & $\mathrm{HR}(95 \% \mathrm{Cl})^{\mathrm{b}}$ & $\mathrm{HR}(95 \% \mathrm{CI})^{\mathrm{C}}$ & $\operatorname{HR}(95 \% \mathrm{CI})^{\mathrm{d}}$ \\
\hline \multicolumn{6}{|l|}{$A P C:$ without $\mathrm{G}>\mathrm{T}$ and $\mathrm{G}>\mathrm{A}$ mutations } \\
\hline T2 & 6484 & 94 & $1.39(1.01-1.92)$ & $1.33(0.96-1.85)$ & $1.34(0.93-1.93)$ \\
\hline T3 & 6480 & 87 & $1.31(0.94-1.82)$ & $1.27(0.91-1.79)$ & $1.28(0.81-2.03)$ \\
\hline$P$-trend & & & 0.13 & 0.20 & 0.36 \\
\hline \multicolumn{6}{|l|}{$A P C: \mathrm{G}>\mathrm{A}$ mutations } \\
\hline T1 & 6496 & 93 & 1 & 1 & 1 \\
\hline $\mathrm{T} 2$ & 6484 & 99 & $1.06(0.79-1.42)$ & $1.03(0.76-1.38)$ & $1.23(0.88-1.73)$ \\
\hline T3 & 6480 & 117 & $1.27(0.96-1.69)$ & $1.21(0.91-1.62)$ & $1.71(1.16-2.50)$ \\
\hline$P$-trend & & & 0.09 & 0.17 & 0.005 \\
\hline $\mathrm{T} 1$ & 6496 & 12 & 1 & 1 & 1 \\
\hline $\mathrm{T} 2$ & 6484 & 9 & $0.75(0.31-1.78)$ & $0.67(0.28-1.62)$ & $0.70(0.26-1.87)$ \\
\hline T3 & 6480 & 19 & $1.59(0.77-3.29)$ & $1.41(0.69-2.88)$ & $1.43(0.53-3.90)$ \\
\hline$P$-trend & & & 0.18 & 0.27 & 0.38 \\
\hline Continuous (1 mg/d intake increment) & & & $1.62(1.02-2.59)$ & $1.52(0.90-2.58)$ & $1.63(0.78-3.40)$ \\
\hline$P$-heterogeneity: $G>T$ in $A P C$ vs. no $G>A$ & and $G>$ & in APC & & & 0.16 \\
\hline \multicolumn{6}{|l|}{ KRAS: without $\mathrm{G}>\mathrm{T}$ and $\mathrm{G}>\mathrm{A}$ mutations } \\
\hline $\mathrm{T} 1$ & 6496 & 137 & 1 & 1 & 1 \\
\hline $\mathrm{T} 2$ & 6484 & 141 & $1.02(0.79-1.31)$ & $0.98(0.76-1.27)$ & $1.02(0.77-1.39)$ \\
\hline$P$-trend & & & 0.05 & 0.10 & 0.01 \\
\hline Continuous (1 mg/d intake increment) & & & $1.25(0.95-1.64)$ & $1.18(0.88-1.58)$ & $1.55(1.06-2.25)$ \\
\hline$P$-heterogeneity: $G>A$ in KRAS vs. no $G>A$ & A and $G$ & in KRAS & & & 0.47 \\
\hline \multicolumn{6}{|c|}{ KRAS: $\mathrm{G}>\mathrm{T}$ mutations } \\
\hline T1 & 6496 & 19 & 1 & 1 & 1 \\
\hline T2 & 6484 & 32 & $1.67(0.94-2.96)$ & $1.62(0.91-2.88)$ & $1.77(0.91-3.44)$ \\
\hline T3 & 6480 & 21 & $1.12(0.60-2.10)$ & $1.07(0.57-2.00)$ & $1.27(0.55-2.94)$ \\
\hline$P$-trend & & & 0.82 & 0.96 & 0.69 \\
\hline Continuous (1 mg/d intake increment) & & & $1.13(0.69-1.86)$ & $1.09(0.67-1.80)$ & $1.36(0.64-2.88)$ \\
\hline \multicolumn{5}{|c|}{$P$-heterogeneity: $G>T$ in KRAS vs. no $G>A$ and $G>T$ in KRAS } & 0.12 \\
\hline
\end{tabular}

Continues on the next page 


\begin{tabular}{|c|c|c|c|c|c|}
\hline \multirow[b]{2}{*}{ Molecular endpoint } & \multirow[b]{2}{*}{ PY } & \multicolumn{4}{|c|}{ Colorectal cancer } \\
\hline & & Cases & $\mathrm{HR}(95 \% \mathrm{Cl})^{\mathrm{b}}$ & $\mathrm{HR}(95 \% \mathrm{Cl})^{\mathrm{c}}$ & $\mathrm{HR}(95 \% \mathrm{Cl})^{\mathrm{d}}$ \\
\hline \multicolumn{6}{|c|}{$A P C$ and $K R A S$ : without $\mathrm{G}>\mathrm{T}$ and $\mathrm{G}>\mathrm{A}$ mutations } \\
\hline T1 & 6496 & 59 & 1 & 1 & 1 \\
\hline $\mathrm{T} 2$ & 6484 & 66 & $1.11(0.77-1.59)$ & $1.06(0.74-1.54)$ & $1.04(0.69-1.57)$ \\
\hline T3 & 6480 & 66 & $1.13(0.79-1.62)$ & $1.11(0.77-1.61)$ & $1.06(0.63-1.78)$ \\
\hline$P$-trend & & & 0.52 & 0.58 & 0.83 \\
\hline Continuous ( $1 \mathrm{mg} / \mathrm{d}$ intake increment) & & & $1.09(0.82-1.46)$ & $1.10(0.80-1.49)$ & $1.05(0.65-1.70)$ \\
\hline \multicolumn{6}{|l|}{$A P C$ and/or KRAS: $\mathrm{G}>\mathrm{A}$ mutations } \\
\hline T1 & 6496 & 111 & 1 & 1 & 1 \\
\hline $\mathrm{T} 2$ & 6484 & 128 & $1.14(0.86-1.49)$ & $1.11(0.85-1.46)$ & $1.30(0.96-1.76)$ \\
\hline T3 & 6480 & 142 & $1.30(1.00-1.69)$ & $1.25(0.86-1.63)$ & $1.68(1.18-2.39)$ \\
\hline$P$-trend & & & 0.05 & 0.10 & 0.03 \\
\hline Continuous ( $1 \mathrm{mg} / \mathrm{d}$ intake increment) & & & $1.15(0.95-1.39)$ & $1.11(0.91-1.35)$ & $1.41(1.09-1.82)$ \\
\hline \multicolumn{5}{|c|}{$\begin{array}{l}\text { P-heterogeneity: } \mathrm{G}>\mathrm{A} \text { in } \mathrm{APC} \text { and/or KRAS vs. no } \mathrm{G}>\mathrm{A} \text { and } \mathrm{G}>\mathrm{T} \text { in } \mathrm{APC} \text { and KRAS } \\
A P C \text { and/or KRAS: } \mathrm{G}>\mathrm{T} \text { mutations }\end{array}$} & 0.84 \\
\hline $\mathrm{T} 1$ & 6496 & 30 & 1 & 1 & 1 \\
\hline $\mathrm{T} 2$ & 6484 & 39 & 1. & 1.22( & 1.26 \\
\hline T3 & 6480 & 40 & $5(0.84-2.19)$ & $1.26(0.78-2.04)$ & $1.32(0.69-2.53)$ \\
\hline$P$-trend & & & 0.23 & 0.35 & 0.44 \\
\hline Continuous ( $1 \mathrm{mg} / \mathrm{d}$ intake increment) & & & $1.33(0.94-1.89)$ & $1.26(0.87-1.83)$ & $1.37(0.79-2.41)$ \\
\hline \multicolumn{5}{|c|}{$P$-heterogeneity: $\mathrm{G}>\mathrm{T}$ in $A P C$ and/or KRAS vs. no $\mathrm{G}>\mathrm{A}$ and $\mathrm{G}>\mathrm{T}$ in $A P C$ and $K R A S$} & 0.78 \\
\hline \multicolumn{6}{|c|}{ 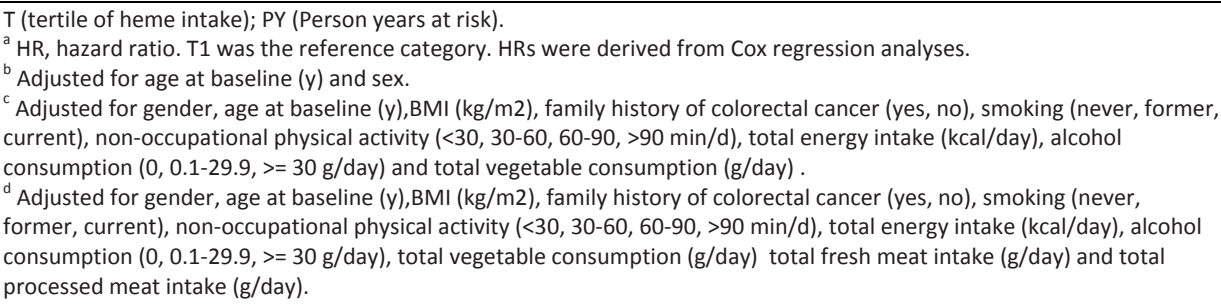 } \\
\hline
\end{tabular}

\section{DISCUSSION}

To the best of our knowledge, this is the first prospective study to report a positive association between heme iron intake and the risk of colorectal tumors harboring $\mathrm{G}>\mathrm{A}$ transitions in KRAS and APC and overexpression of P53.

Heme iron intake was dose-dependently associated with an increased risk of colorectal cancer with KRAS codon 12 and 13 mutations but not with wild type KRAS tumors. More specifically, we observed a clear dose-response relation between heme iron consumption and colorectal cancers with specific $\mathrm{G}>\mathrm{A}$ activating transitions in KRAS. Similar findings were observed with overall G>A mutations in $A P C$, which is in line with a recent case only study from EPIC-Norfolk [26]. These G>A transitions are characteristic for alkylating agents such as NOC or their metabolites [15]. The endogenous formation of NOC is catalyzed by heme [13]. Some NOC have shown to react with DNA in vitro to give rise to the NOC specific DNA adducts such as 06-carboxymethyldeoxyguanine (O6CMG) [14], which was increased in exfoliated colonic cells from human volunteers who were fed a high red meat diet for fifteen days [48]. O6CMG adducts may cause DNA polymerase to misread the O6-guanine as an adenine which results in miss-pairing with thymine, causing $G>A$ transitions [49]. Supporting these data, N-methyl N-nitrosourea, a potent and direct acting NOC, has shown to induce $\mathrm{G}>\mathrm{A}$ transitions at codons 12 and 13 of the KRAS gene in rat colon tumors when infused intrarectally [16]. 
Heme iron also catalyses the formation of hydroxyl radicals that can cause oxidative DNA lesions $[18,19]$ which can promote $G>T$ transversions [20]. Moreover, NOC-metabolism has been shown to generate reactive oxygen species, which was associated with the deregulation of gene expression patterns that may be of relevance in human colorectal carcinogenesis (18). However, in both KRAS and APC the magnitude of our findings for $\mathrm{G}>\mathrm{T}$ mutations was smaller as compared to our $G>A$ specific associations, if present at all. Although the tests for heterogeneity between these molecular subgroups were not statistically significant, this may imply that the heme stimulated alkylating effect of NOC might play a larger role in colorectal carcinogenesis than its oxidative potential. Indeed, it has been proposed previously that DNA alkylation is probably the most relevant genotoxic effect induced by NOC [50]. However, G>A mutations can, next to alkylating damage, also result from other endogenous mechanisms. Nonetheless, the number of subjects with $G>A$ and $\mathrm{G}>\mathrm{T}$ mutations was relatively small and more research in larger samples is required.

Although heme iron intake was not significantly associated with the risk of tumors with (specific) mutations leading to the introduction of a stopcodon in $A P C$, a significant positive trend between heme iron intake and tumors without an APC stopcodon was observed. These findings are supported by epidemiological studies examining red meat intake in relation to APC truncation status in the NLCS [28] and a Dutch colorectal adenoma case control study [51], but contradict those from a cancer case control study [52] and a case only study [26].

The initial focus of our analyses was on functional mutations; inactivation of the APC gene and activation of KRAS are thought to drive the development of a carcinoma and are considered key events in early colorectal tumorigenesis [53]. The potential impact of nonfunctional mutations in colorectal cancer development is not yet understood. Nevertheless, we only observed an association between heme iron intake and APC tumors without the introduction of a stopcodon. However, only half of all APC mutations led to the introduction of such a stopcodon which prompted us to examine the group of tumors harboring any APC mutation more closely for specific point mutation status, regardless of its functionality. These analyses showed an increased risk for tumours harbouring overall $G>A$ mutations in APC which was not found when restricting to functional mutations only. This could suggest that the carcinogenic mechanism behind heme intake involves exposurespecific mutations that may not necessarily entail inactivation of $A P C$. The wide spectrum of the $A P C$ mutation cluster region mutations could, in contrast to codon 12 and 13 mutations in the KRAS gene (harbouring mainly $\mathrm{G}$ changes), serve as a potential useful fingerprint for long term exposure to dietary heme iron. However, these conclusions are speculative and our findings need to be reproduced in future research.

In line with previous experimental evidence, we showed that heme iron intake was positively associated with the risk of P53 overexpressed colorectal tumors. However, because the absence of P53 overexpression does not necessarily imply the absence of TP53 mutations, and P53 overexpression may occur in the absence of mutations and vice versa $[54,55]$ we are prudent when interpreting these findings.

The observed risk estimates were weaker and more unstable in rectum cases compared to colon cases. Although this may partly be attributed to the lower number of rectum cancer 
cases, previous studies suggest that dietary risk factors for colon and rectum cancer may differ as a result of, for instance, physiologic and biochemical sub-site differences $[9,56]$ that could affect the carcinogenic potential of meat mutagens such as heme iron. Nonetheless, tests for heterogeneity suggest that there is no statistical heterogeneity between the rectum and the colon endpoint, and results should be interpreted with caution.

The present study illustrates that when unraveling the mechanisms underlying the association between heme iron and colorectal cancer risk, it is valuable to take both 1) specific meat carcinogens such as heme iron, and 2) the molecular heterogeneity of the colorectal endpoint into account. Failure to do so may result in attenuated risk estimates as reflected in previous analyses from our cohort [7, 27, 28].

Remarkably, the associations presented herein were stronger after additional adjustment for fresh and processed meat intake. This may be explained by the previously reported suggestive inverse associations between total meat intake, particularly pork consumption, and the risk of colon and rectal cancer harboring specific mutations in this population [27, 28]. However, heme iron captures the variation in red meat intake better than pork consumption alone, which is consumed at relatively high amounts in our population. By adjusting the associations for total meat intake we attempted to partly adjust for all other mechanism by which meat intake may cause colorectal cancer, and our findings may indeed suggest that heme iron itself is an important player in meat induced carcinogenesis. In addition, when adjusting our analyses for total meat intake, an increase in heme iron intake results from a displacement in the composition of total fresh meat intake (e.g. pork to beef ratio). If other characteristics (e.g. cooking methods) differ for specific subtypes of meats and are relevant to colorectal cancer, this could result in residual confounding. In addition, other dietary characteristics specific to high heme iron consumers that are not captured by the covariates in the model could also result in residual confounding.

Our heme iron database was based on the total iron content of each meat item in the Dutch food composition database whereby an animal-specific proportion of heme iron relative to total iron was applied, as derived from analytical data reported in the literature for cooked meats. Heme iron values were available for all meat items in the FFQ, including specific Dutch items and cuts. Nevertheless, our method fails to account for effect of the cooking- method and duration on the conversion of heme iron to non heme iron; information that is available in the database developed at $\mathrm{NCl}[57,58]$. To evaluate the accuracy of our heme iron estimates, we compared the heme iron concentrations of meat items from our database to the $\mathrm{NCl}$ database, for those meat items for which an American equivalent was available [58]. The concentrations heme iron in pork, chicken and processed meat were comparable across both databases but the heme iron content of beef items (i.e. steak and hamburger) was considerable higher in the NLCS database.

We were not able to account for other, possibly correlated, meat mutagens such as heterocyclic amines (HCAs) and polycyclic aromatic hydrocarbons (PAHs) which are formed in meats when cooked well-done at high temperatures $[59,60]$. Although both groups of mutagens have shown to be implicated in DNA-adduct formation $[61,62]$ and colorectal carcinogenesis [59], they cannot explain the differential carcinogenic effect of white and 
red meat in the colon. Also a recent dietary intervention study with red meat showed that the observed increase in fecal genotoxicity was not likely to be explained by increased levels of HCAs or PAHs, but most likely due to heme iron mediated processes [63].

Our analyses have been performed using baseline FFQ data, resulting in an inability to assess and account for changes in intake and food compositions over time. However, the FFQ has shown to be representative for dietary habits over a period of at least 5 years [38]. The prospective design reduced the potential for recall bias, and the nearly complete follow-up of cases and subcohort members, makes selection bias unlikely. Detailed information on diet and potential risk factors of colorectal cancer enabled us to control for most known risk factors, although misclassification of exposure may have occurred. Finally, although our results support prior biological hypotheses we cannot rule out the possibility of chance findings given the relatively small subgroups of mutation specific colorectal tumors and the numerous associations investigated.

In conclusion, this is the first cohort study reporting the association between heme iron intake and the risk of colorectal cancer harboring specific mutations in key genes. Our results suggest that heme iron intake is especially associated with an increased risk of colorectal tumors harboring G>A transitions in KRAS and APC and overexpression of P53. These novel findings suggest that alkylating rather than oxidative DNA damaging mechanisms are involved in heme-induced colorectal carcinogenesis.

\section{REFERENCES}

1. World Cancer Research Fund/American Institute for Cancer Research, Food, Nutrition, Physical Activity, and the Prevention of Cancer: a Global Perspective. 2007, AICR: Washington DC.

2. Larsson, S.C. and A. Wolk, Meat consumption and risk of colorectal cancer: a meta-analysis of prospective studies. Int J Cancer, 2006. 119(11): p. 2657-64.

3. Chan, D.S., et al., Red and processed meat and colorectal cancer incidence: meta-analysis of prospective studies. PLoS One, 2011. 6(6): p. e20456.

4. Service., U.S.D.o.A.a.A.R., USDA National Nutrient Database for Standard Reference, Release 21. Internet: Nutrient Data Laboratory Home Page, http://www.ars.usda.gov/nutrientdata (accessed 22 November 2011).

5. Larsson, S.C., et al., Re: Heme iron, zinc, alcohol consumption, and risk of colon cancer. J Natl Cancer Inst, 2005. 97(3): p. 232-3; author reply 233-4.

6. Lee, D.H., et al., Heme iron, zinc, alcohol consumption, and colon cancer: lowa Women's Health Study. J Natl Cancer Inst, 2004. 96(5): p. 403-7.

7. Balder, H.F., et al., Heme and chlorophyll intake and risk of colorectal cancer in the Netherlands cohort study. Cancer Epidemiol Biomarkers Prev, 2006. 15(4): p. 717-25.

8. Kabat, G.C., et al., A cohort study of dietary iron and heme iron intake and risk of colorectal cancer in women. Br J Cancer, 2007. 97(1): p. 118-22.

9. Cross, A.J., et al., A large prospective study of meat consumption and colorectal cancer risk: an investigation of potential mechanisms underlying this association. Cancer Res, 2011. 70(6): p. 2406-14.

10. Zhang, X., et al., A prospective study of intakes of zinc and heme iron and colorectal cancer risk in men and women. Cancer Causes Control, 2011. 22(12): p. 1627-37.

11. Bastide, N.M., F.H. Pierre, and D.E. Corpet, Heme iron from meat and risk of colorectal cancer: a metaanalysis and a review of the mechanisms involved. Cancer Prev Res (Phila), 2011. 4(2): p. 177-84.

12. Fearon, E.R., Molecular genetics of colorectal cancer. Annual review of pathology, 2011. 6: p. 479-507.

13. Cross, A.J., J.R. Pollock, and S.A. Bingham, Haem, not protein or inorganic iron, is responsible for endogenous intestinal N-nitrosation arising from red meat. Cancer Res, 2003. 63: p. 2358-2360.

14. Shuker, D.E. and G.P. Margison, Nitrosated glycine derivatives as a potential source of O6methylguanine in DNA. Cancer Res, 1997. 57(3): p. 366-9. 
15. Saffhill, R., G.P. Margison, and P.J. O'Connor, Mechanisms of carcinogenesis induced by alkylating agents. Biochim Biophys Acta, 1985. 823(2): p. 111-45.

16. Jacoby, R.F., et al., K-ras oncogene mutations in rat colon tumors induced by N-methyl-N-nitrosourea. Carcinogenesis, 1992. 13(1): p. 45-9.

17. Gottschalg, E., et al., Potassium diazoacetate-induced p53 mutations in vitro in relation to formation of 06-carboxymethyl- and 06-methyl-2'-deoxyguanosine DNA adducts: relevance for gastrointestinal cancer. Carcinogenesis, 2007. 28(2): p. 356-62.

18. Hebels, D.G., et al., Radical mechanisms in nitrosamine- and nitrosamide-induced whole-genome gene expression modulations in Caco-2 cells. Toxicol Sci, 2010. 116(1): p. 194-205.

19. Tappel, A., Heme of consumed red meat can act as a catalyst of oxidative damage and could initiate colon, breast and prostate cancers, heart disease and other diseases. Med Hypotheses, 2007. 68(3): p. 562-4.

20. Shibutani, S., M. Takeshita, and A.P. Grollman, Insertion of specific bases during DNA synthesis past the oxidation-damaged base 8-oxodG. Nature, 1991. 349(6308): p. 431-4.

21. Sesink, A.L., et al., Red meat and colon cancer: the cytotoxic and hyperproliferative effects of dietary heme. Cancer Res, 1999. 59(22): p. 5704-9.

22. Sesink, A.L.A., et al., Red meat and colon cancer: Dietary haem, but not fat, has cytotoxic and hyperproliferative effects on rat colonic epithelium. Carcinogenesis, 2000. 21: p. 1909-1915.

23. IJssennagger, N., et al., Dietary haem stimulates epithelial cell turnover by downregulating feedback inhibitors of proliferation in murine colon. Gut, 2012. 61(7): p. 1041-9.

24. Ahearn, T.U., et al., Markers of the APC/beta-catenin signaling pathway as potential treatable, preneoplastic biomarkers of risk for colorectal neoplasms. Cancer epidemiology, biomarkers \& prevention : a publication of the American Association for Cancer Research, cosponsored by the American Society of Preventive Oncology, 2012. 21(6): p. 969-79.

25. Naguib, A., et al., Dietary, lifestyle and clinicopathological factors associated with BRAF and K-ras mutations arising in distinct subsets of colorectal cancers in the EPIC Norfolk study. BMC cancer, 2010. 10: p. 99.

26. Gay, L.J., et al., Dietary, lifestyle and clinicopathological factors associated with APC mutations and promoter methylation in colorectal cancers from the EPIC-Norfolk study. The Journal of pathology, 2012. 228(3): p. 405-15.

27. Brink, M., et al., Meat consumption and K-ras mutations in sporadic colon and rectal cancer in The Netherlands Cohort Study. Br J Cancer, 2005. 92: p. 1310-1320.

28. Lüchtenborg, M., et al., Meat and fish consumption, APC gene mutations and hMLH1 expression in colon and rectal cancer: a prospective cohort study (The Netherlands). Cancer Causes Control, 2005. 16: p. 1041-1054.

29. Park, J.Y., et al., Lifestyle factors and p53 mutation patterns in colorectal cancer patients in the EPICNorfolk study. Mutagenesis, 2010. 25(4): p. 351-8.

30. Freedman, A.N., et al., Familial and nutritional risk factors for p53 overexpression in colorectal cancer. Cancer Epidemiol Biomarkers Prev, 1996. 5(4): p. 285-91.

31. Voskuil, D.W., et al., p53 over-expression and p53 mutations in colon carcinomas: relation to dietary risk factors. International journal of cancer. Journal international du cancer, 1999. 81(5): p. 675-81.

32. Sesink, A.L., et al., Red meat and colon cancer: dietary haem-induced colonic cytotoxicity and epithelial hyperproliferation are inhibited by calcium. Carcinogenesis, 2001. 22(10): p. 1653-9.

33. de Vogel, J., et al., Green vegetables, red meat and colon cancer: chlorophyll prevents the cytotoxic and hyperproliferative effects of haem in rat colon. Carcinogenesis, 2005. 26(2): p. 387-93.

34. Van den Brandt, P.A., et al., A large-scale prospective cohort study on diet and cancer in The Netherlands. J Clin Epidemiol, 1990. 43: p. 285-295.

35. Goldbohm, R.A., P.A. van den Brandt, and E. Dorant, Estimation of the coverage of Dutch municipalities by cancer registries and PALGA based on hospital discharge data. Tijdschrift Soc Gezondheidsz, 1994. 72: p. 80-84.

36. Nevo table: Dutch Food Composition Table 1986-1987 (in Dutch). 1986, The Hague: Voorlichtingsbureau voor de voeding.

37. Goldbohm, R.A., et al., Validation of a dietary questionnaire used in a large-scale prospective cohort study on diet and cancer. Eur J Clin Nutr, 1994. 48(4): p. 253-65.

38. Goldbohm, R.A., et al., Reproducibility of a food frequency questionnaire and stability of dietary habits determined from five annually repeated measurements. Eur J Clin Nutr, 1995. 49(6): p. 420-9. 
39. Brink, M., et al., K-ras oncogene mutations in sporadic colorectal cancer in The Netherlands Cohort Study. Carcinogenesis, 2003. 24: p. 703-710.

40. Luchtenborg, M., et al., APC mutations in sporadic colorectal carcinomas from The Netherlands Cohort Study. Carcinogenesis, 2004. 25(7): p. 1219-26.

41. Bongaerts, B.W.C., et al., Alcohol consumption and distinct molecular pathways to colorectal cancer. British Journal of Nutrition, 2007. 97(03): p. 430-434.

42. Schouten, L.J., J.J. Jager, and P.A. van den Brandt, Quality of cancer registry data: a comparison of data provided by clinicians with those of registration personnel. British journal of cancer, 1993. 68(5): p. 974-7.

43. Volovics, A. and P.A. van den Brandt, Methods for the analysis of case-cohort studies. Biom J, 1997(39): p. 195-214.

44. Lin, D.Y. and L.J. Wei, The robust inference for the Cox proportional hazards model. J AM Stat Assoc, 1989(84): p. 1074-8.

45. Wacholder, S., et al., Alternative variance and efficiency calculations for the case-cohort design. Biometrika, 1989. 76(1): p. 117-23.

46. de Vogel, S., et al., Associations of dietary methyl donor intake with MLH1 promoter hypermethylation and related molecular phenotypes in sporadic colorectal cancer. Carcinogenesis, 2008. 29(9): p. 1765-73.

47. Willett W., Nutritional Epidemiology. 1998, New York: Oxford University Press.

48. Lewin, M.H., et al., Red meat enhances the colonic formation of the DNA adduct O6-carboxymethyl guanine: implications for colorectal cancer risk. Cancer Res, 2006. 66(3): p. 1859-65.

49. Horsfall, M.J., et al., Mutational specificity of alkylating agents and the influence of DNA repair. Environ Mol Mutagen, 1990. 15(2): p. 107-22.

50. Bartsch, H., E. Hietanen, and C. Malaveille, Carcinogenic nitrosamines: free radical aspects of their action. Free Radic Biol Med, 1989. 7(6): p. 637-44.

51. Diergaarde, B., et al., Dietary factors and truncating APC mutations in sporadic colorectal adenomas. Int J Cancer, 2005. 113(1): p. 126-32.

52. Diergaarde, B., et al., Dietary factors and the occurrence of truncating APC mutations in sporadic colon carcinomas: a Dutch population-based study. Carcinogenesis, 2003. 24(2): p. 283-90.

53. Fearon, E.R. and B. Vogelstein, A genetic model for colorectal tumorigenesis. Cell Mol Life Sci, 1990. 61: p. 759-767.

54. Dix, B., et al., Comparison of p53 gene mutation and protein overexpression in colorectal carcinomas. Br J Cancer, 1994. 70(4): p. 585-90.

55. Cripps, K.J., et al., A study of stabilisation of p53 protein versus point mutation in colorectal carcinoma. Oncogene, 1994. 9(9): p. 2739-43.

56. Povey, A.C., et al., Elevated levels of the pro-carcinogenic adduct, O(6)-methylguanine, in normal DNA from the cancer prone regions of the large bowel. Gut, 2000. 47(3): p. 362-5.

57. Ferrucci, L.M., et al., Dietary meat intake in relation to colorectal adenoma in asymptomatic women. Am J Gastroenterol, 2009. 104(5): p. 1231-40.

58. Cross, A. and R. Sinha, Developing a heme iron database for meats according to meat type, cooking method and doneness level. Food and Nutrition Sciences In press.

59. Cross, A.J. and R. Sinha, Meat-related mutagens/carcinogens in the etiology of colorectal cancer. Environ Mol Mutagen, 2004. 44(1): p. 44-55.

60. Sinha, R., et al., Development of a food frequency questionnaire module and databases for compounds in cooked and processed meats. Mol Nutr Food Res, 2005. 49(7): p. 648-55.

61. Dingley, K.H., et al., DNA and protein adduct formation in the colon and blood of humans after exposure to a dietary-relevant dose of 2-amino-1-methyl-6-phenylimidazo[4,5-b]pyridine. Cancer Epidemiol Biomarkers Prev, 1999. 8(6): p. 507-12.

62. Turteltaub, K.W., et al., Macromolecular adduct formation and metabolism of heterocyclic amines in humans and rodents at low doses. Cancer Lett, 1999. 143(2): p. 149-55.

63. Hebels, D.G., et al., Red meat intake-induced increases in fecal water genotoxicity correlate with procarcinogenic gene expression changes in the human colon. Food Chem Toxicol, 2012. 50(2): p. 95-103. 



\section{CHAPTER 10}

General discussion 
In the previous chapters we described a series of studies aimed at comprehensively investigating the association between meat consumption and cancer risk focusing on meatrelated dietary patterns and specific meat-related carcinogenic pathways. This chapter contains a discussion of the most important findings in light of some important strengths and weaknesses of the study designs and analyses. Furthermore, recommendations for future epidemiological research are given.

\section{MAIN FINDINGS}

In part A of this thesis, we focused our analyses on meat consumption as part of a dietary pattern. This section started with two studies on meat consumption patterns and noncancer health outcomes (i.e. weight change and risk of vitamin B12 and folate deficiency), since these may affect or explain some of the association between vegetarian and low meat diets and cancer risk. Although baseline BMI values were strongly positively associated with meat intake, we found no association between total meat intake and changes in BMI over 14 years of prospective follow-up in the Netherlands Cohort Study (NLCS). Nonetheless, modest differential BMI change effects were observed for some subtypes of meat. In the EPIC-Oxford study, we showed that vegetarians, and especially vegans, were at an increased risk of developing a vitamin B12 deficiency. Because low meat consumers are likely at a reduced risk of developing nutrient deficiencies, we subsequently investigated if they also had an additional beneficial cancer preventive effect over vegetarians when compared to individuals who consume meat on a daily basis. In order to study how a vegetarian or low meat diet (i.e. meat 1 day/week) influences the risk of cancer compared to a more common high meat diet, we extracted the NLCS-Meat Investigation Cohort (NLCS-MIC) from the total NLCS cohort. In line with our expectations, a baseline comparison of this cohort indicated that vegetarians, pescetarians, and individuals consuming meat for 1 day/week had a more favorable distribution of (non-)dietary cancer risk factors (e.g. high intakes of fruit and vegetables, lower smoking rates) compared to regular meat consumers. Additionally, we observed that as much as $50 \%$ of all the individuals who classified themselves as vegetarian (based on 1 item on the first page of the questionnaire) reported (some) meat or fish consumption on the extensive FFQ. However, the misclassification that occurred in terms of baseline diet (other than meat) and lifestyle characteristics using these self-reports was relatively small.

Our hypothesis that low meat diets result in a similar or perhaps even a lower cancer risk than vegetarian diets when compared to a high meat diet could only partly be verified. Compared to high meat consumers (defined as individuals who eat meat for 6-7 days/week), both non- and low meat consumers had a non-significantly decreased risk of colorectal, especially rectal, cancer which attenuated after adjustment for confounders. In age and sex adjusted models, a decreased risk of lung cancer was observed for vegetarians and pescetarians, but this effect disappeared after taking confounders, especially smoking, into account. No associations between meat consumption group and female breast cancer were observed, while low meat consumers had an increased risk of advanced prostate cancer. However, this latter observation is likely due to chance. As a result of our sampling strategy, we had a wide distribution of meat intake in the NLCS-MIC compared to many other population-based studies. This prompted us to also examine the association between individual (sub) types of meat and cancer risk next to the previously described dietary 
pattern analyses. In these analyses, we did not observe clear associations between total fresh and fresh red meat intake and any of the investigated cancers. However, high consumption of processed meat was associated with an increased risk of rectal cancer, and an unexpected reduced risk of advanced prostate cancer.

In part B of this thesis, we investigated several of the proposed meat-related carcinogenic pathways in relation to the cancer endpoints most likely to be affected. Contrary to our hypothesis that the high fat content from meat may be responsible for the meat-cancer association, no clear evidence for an association between dietary fat and meat intake and ovarian cancer risk was found in the NLCS. Although trans unsaturated fatty acids were associated with an increased risk of ovarian cancer, the majority of this type of fat at the time of the baseline assessment, does not originate from meat, but stems from margarines, packaged baked products and fast foods. In addition, consumption of heterocyclic amines (HCAs), polycyclic aromatic hydrocarbons (PAHs), and nitrite/nitrate was not strongly associated with the risk of colorectal adenomas and colorectal cancer in the PLCO Screening Trial. By using novel data reduction techniques (i.e. a two-step test for G×E interactions) we found evidence that a genetic variant in the NAT1 gene modified the effect of MelQx intake on colorectal adenoma risk. Additional effect modification of the association between other compounds and colorectal adenoma and cancer risk by variants in other xenobiotic metabolizing enzymes (XME) genes were also observed. However correction for multiple comparisons indicated that these latter were likely due to chance. In our next study, we found supportive evidence for the role of heme iron in colon carcinogenesis in the NLCS. Our analyses suggest that heme iron probably results in alkylating DNA damage in the colon by initiating specific $\mathrm{G}>\mathrm{A}$ transitions in several colorectal cancer key genes (e.g. APC and KRAS).

\section{METHODOLOGICAL CONSIDERATIONS}

Because the study of vegetarian and low meat diets can introduce specific sources of bias that are less likely to occur when studying individual food items, this section will highlight several methodological issues.

\section{Selection bias}

It is unlikely that selection bias has distorted the results presented in this thesis. Selection bias due to selection of the study population in a cohort study as the NLCS (including the NLCS-MIC) and EPIC-Oxford is unlikely because the entire population is disease free at baseline. Selection bias in prospective cohort studies may occur when participants are lost to follow up and when this depends on exposure and future outcome status [1]. However, in the NLCS the completeness of cancer follow-up was estimated to be $>96 \%[2,3]$ and less than $1 \%$ of the cohort members were lost to follow-up for vital status, making selection bias very unlikely. Vegetarians were overrepresented to increase the meat exposure contrast in the NCLS, but we do not expect that this has introduced selection bias. The colorectal adenoma and cancer analyses in the screening arm of the PLCO trial were conducted in two separate nested case-control studies. The nested design and the screening nature of the trial minimized the chance of selection bias, since both cases and controls had an equal opportunity to have a colorectal adenoma or cancer detected. In 
addition, we only selected advanced adenoma cases to reduce length-time bias associated malignancy differences between adenomas.

\section{Information bias}

All the studies included in this thesis used food frequency questionnaires to estimate habitual dietary intake. While faced with certain limitations [4], this is often the most practical and economical method of dietary data collection in large scale epidemiological studies [5]. Although the FFQs used in the NLCS, the EPIC-Oxford cohort, and the PLCO screening trial were specifically designed or adapted for use in their respective population, they are still liable to error which may have led to several sources of misclassification. However, all the types of misclassification that could have occurred are non-differential, meaning that the extent of misclassification does not depend on disease and exposure status.

FFQs are designed to measure long term habitual food intake which is considered the conceptually important exposure in etiological studies of cancer [5]. It is, however, likely that respondents are unable to exactly report their average consumption of foods (i.e. meat), resulting in misclassification of exposure [5]. However, when studying the association between diet and cancer, the ranking of subjects according to their dietary intake is more important than estimating their absolute intake level. All FFQs used in this thesis were validated against some independent superior reference method. For all prospective analyses presented in this thesis (Chapters 2, 4-9), the information on dietary intake and lifestyle was only ascertained at baseline. However, the questionnaire used in the NLCS was shown to be representative for dietary habits over a period of at least 5 years [6] whereas the FFQ used in the PLCO Screening trial was adapted from the Willet and Block FFQs with moderate to good reliability $[7,8]$.

There are additional sources of misclassification that could have arisen specific to the study of meat and its associations with cancer. Firstly, the categorization and analysis of meat differs between epidemiological studies. Illustrative examples are red meat and processed meat [9]. This issue is to some extent also reflected in this thesis, which includes studies from the Netherlands, the United Kingdom and the USA. Although in each study it was explicitly defined what was meant by red and processed meat, definitions and consumption patterns vary by population, which can complicate between-study comparisons. For example, in contrast to many other populations, the generation of the NLCS generally eats two cold meals a day (for breakfast and lunch) consisting of bread served with a wide variety of cold cuts. The types of processed meat consumed also likely differ between these three study populations. In addition, the majority of red meat consumed in the Netherlands and the United Kingdom consists of pork, whereas beef makes up most of red meat in the USA [10]. As a result, the composition of the red and processed meat variable in the NLCS is probably different from that in the PLCO screening trial and the EPIC-Oxford cohort, which should be taken into consideration when reviewing the literature.

Secondly, there is no universally accepted definition of vegetarianism. The term vegetarian is often used to describe a whole range of diets practiced with varying degrees of animal product restriction [11]. In addition, there is substantial inconsistency in how people selfidentify being vegetarian $[12,13]$. This challenges the comparison of between-study results. 
For this reason, we defined vegetarians in the NLCS-MIC using two methods: based on selfdefinition and based on their response on the extensive FFQ. Assuming that the latter method is most accurate; a cross-comparison suggests that self-definition severely overestimated the prevalence of vegetarianism in our population. Although our power was somewhat limited, our results suggest that self-defined vegetarian status may attenuate risk estimates in etiologic studies of cancer risk.

Finally, difficulties in quantifying the intake of specific meat mutagens can introduce additional information bias. The study of most meat carcinogens is limited to studies that have inquired both after 1) type or cut of meat, 2) cooking technique, and 3) doneness level [14]. However, many of the early prospective cohort studies, including the NLCS, did not incorporate such a detailed meat module in their questionnaire. From the mid-nineties of the last century, efforts were made to develop a validated meat-cooking FFQ and meat carcinogen database to estimate the intake of HCAs, PAHs, and nitrite/nitrate in epidemiological studies [14-17]. Because meat preparation and consumption patterns vary greatly between populations, we used a population specific meat cooking module for the analysis described in Chapter 8. In contrast, cooking methods do not seem to have a large effect on the heme iron content of the meat; a comparison between the literature based NLCS database used in Chapter 9 and a database containing measured values from cooked meats developed by $\mathrm{NCl}$ [18] generally yielded comparable concentrations.

In the molecular epidemiological studies reported herein (Chapter 8 and 9), there is the additional problem of possible measurement error and misclassification of genotype and molecular pathological endpoint. It is therefore important to use validated molecular markers and perform quality control measurements $[19,20]$. In addition, we excluded individuals with genotyping call rates below $90 \%$ in the PLCO screening trial (Chapter 8), and cases with poor quality tumor material (i.e. insufficient DNA) in the NLCS (Chapter 9) from all presented analyses.

At the time of exposure measurement all the populations under study were disease free except for the prevalent adenoma study in the PLCO screening trial. However, adenomas are largely asymptomatic, and at the time of the questionnaire respondents did not know the results of their colonoscopy. Therefore, this has likely not resulted in differential reporting of meat consumption in this population. All the above listed sources of nondifferential misclassification tend to bias the effect estimate to the null [5] and can lead to attenuation of the true meat-cancer association. This may have resulted in an underestimation of the observed effects and might partially explain some of the observed null-results.

\section{Confounding}

Although all observational studies in general should adequately adjust for confounding variables in analyses in order to prevent bias [1], issues relating to confounding are of particular interest when studying the health effects of vegetarian and low meat diets. A common criticism of the causal interpretation of observational studies investigating the association between vegetarian diets and cancer risk is that these populations are likely to exhibit a number of other potential cancer preventive factors and behaviors next to the (near) avoidance of meat. This can result in different levels of confounding ranging from a 
more beneficial lifestyle and a healthier diet to more subtle self-selection processes, as described below.

Firstly, vegetarians and low meat consumers are committed to a general healthy lifestyle. This is reflected in a beneficial distribution of non-dietary factors including physical activity, BMI, alcohol consumption and smoking which was also observed in the NLCS-MIC. However, most prior studies examining cancer risk in vegetarians have controlled for confounding by some but not all of these factors. The Adventist Health Study had the unique opportunity to study cancer risk of vegetarians independent of the effect of smoking and alcohol consumption since both the vegetarians and meat consumers in this population were generally non users [21]. In the NLCS-MIC, correction for all the above mentioned lifestyle factors resulted in an attenuation of the association of vegetarians and low meat consumers. This was especially true for lung cancer, where the lower risk among vegetarians compared to high meat eaters disappeared after adjusting for smoking status, duration and to a lesser extent, frequency.

Secondly, we showed that vegetarian and low meat diets include larger amounts of fruits, vegetables, and fiber. Although none of these food groups are unique to these diets, the (near) avoidance of meat automatically allows for more plant based food in the diet. An abundant consumption of many of these products has been independently related with a lower risk for several cancers [9]. This raises the possibility that a high plant-food dietary pattern may be the true causal protective factor rather than the simple elimination of meat from the diet. For this reason, we set out to investigate to what extent differences in cancer risk, if present, could (partially) be attributed by confounding dietary variables other than meat intake. We observed that, if an association was found, fiber (and to a lesser extent fruits and soy products) explained most of the between group risk estimates. This observation, however, may result from multicollinearity with other (un)measured dietary variables that characterize a low and no meat diet. Dietary fiber intake may also simply be a good overall marker for the vegetarian and low meat diet and adjusting for this may have resulted in overcorrection of the risk estimates. Nevertheless, all other dietary factors that characterize a vegetarian and low meat diet had negligible individual effects, but when combined they changed the risk estimates considerably. This illustrates that the combined effect of the entire vegetarian and low meat diet may be more powerful than the effects of its individual components, and thus more readily detected.

Lastly, it has been suggested that the lower disease risk in vegetarians compared to nonvegetarians may be explained by selective factors related to who chooses to become and remain a vegetarian [22, 23]. Vegetarians are suggested to have different social backgrounds and education compared to meat eaters, and may have taken up their dietary habit because they are health-conscious. This may also lead to different health care seeking behavior. In addition, prior illness may have prompted individuals to adopt a diet void of meat. In the NLCS-MIC, we showed that the proportion of prevalent cancer cases was highest among the vegetarians and pescetarians and significantly decreased with increasing meat intake. This suggests that self-selection mechanisms should be taken into consideration in etiologic studies. Compared to the two previously mentioned classes of confounding, it is more difficult to accurately adjust for these subtle selective factors in analyses. Therefore, we excluded all prevalent cancer cases from analysis and adjusted the 
risk estimates for level of education. When similar cancer risks are observed across different populations of vegetarians it is more likely that the results can be attributed to the diet and lifestyle as such rather than any underlying selection processes. Although other studies on low meat consumers are scarce, findings from the NLCS-MIC were generally comparable to the results from other prospective studies investigating vegetarian diets in relation to colorectal, lung, female breast and overall prostate cancer risk [21, 2427]; after correction for confounders no large differences in cancer risk were observed between vegetarians and non-vegetarians/high meat consumers.

Depending on the specific research question of interest, adjustment for one or more of these three levels of confounding (i.e. lifestyle, diet, and selective factors) is required. For example, studies comparing vegetarian to meat eating populations that are mainly concerned with the adverse effects of meat on cancer risk, warrant adjustment for all three levels in order to extract the individual effect of meat consumption. However, it is equally interesting to study the complete vegetarian diet or vegetarian lifestyle in relation to disease. For this, one would suffice by adjusting for lifestyle and selective factors or selective factors only, respectively. Nonetheless, if an association with a dietary pattern or lifestyle is found, one does not know which factor(s) are (primarily) responsible for the observed effects. This lack of specificity likely leads to broad, less focused, and potentially less effective cancer preventive recommendations. Within the NLCS-MIC we were able to study both research questions by adjusting our analysis for multiple levels of confounding. Although somewhat underpowered, our study suggests that both dietary and non-dietary factors associated with low and no meat diets contribute to differences in colorectal, lung, and prostate cancer risk.

\section{BIOLOGICAL PLAUSIBILITY}

Because studying dietary patterns does not enhance biological insight or mechanistic understanding of the meat cancer association [28], we have investigated several of the hypothesized pathways of meat-related carcinogenesis (dietary fat, HCAs, PAHs, nitrate/nitrite, and heme iron) in the second part of this thesis. Where possible, we specified these associations for certain genetic variants or molecular pathological changes to provide further evidence for a causal relationship between specific types of meat (carcinogens) and cancer.

\section{Dietary fat and ovarian cancer risk}

We did not find evidence that fat from fresh and processed meats increased the risk of ovarian cancer (RR 1SD increase $=0.98 ; 95 \% \mathrm{Cl}$ : 0.87-1.09). Neither did we find a clear dose response association for animal fat, saturated fat, or total fat which may be present in high quantities in a meat-rich diet. Since the publication of our results, the AARP cohort (including nearly 700 cases) reported that fat intake from animal sources was positively associated with ovarian cancer risk (RRQ5 vs Q1=1.30; 95\% Cl: 1.02-1.66) [29]. This discrepancy in findings may partly result from the wider range of dietary fat intake in the AARP population (USA) (percent energy intake from fat p10: $20 \%$; p90: $40 \%$ ) compared to the Dutch in the NLCS (p10: 32\%; p90: 46\%). The Women's Health Initiative Dietary Modification Trial suggested that a low fat dietary pattern (24.3\% compared to $35.1 \%$ energy from fat after 1 year from baseline for the intervention and control group, 
respectively) may reduce the incidence of ovarian cancer in post-menopausal women [30]. Nonetheless, the overall epidemiological evidence linking dietary (animal) fat to ovarian cancer remains inconsistent and is not strongly supportive of the dietary fat hypothesis of meat-related carcinogenesis for this cancer endpoint [9]. The absence of such a clear association is in line with a meta-analysis of eight cohort studies (including ours) that did not observe a dose-response relation between red and processed meat intake and the risk of cancer of the ovaries [31].

\section{GXE interactions, meat carcinogens, and colorectal neoplasia}

Despite the strong carcinogenic potential of individual HCAs, (PhiP, MelQx, and DiMelQx), $\mathrm{PAH}(\mathrm{B}[\mathrm{a}] \mathrm{P})$, and nitrite/nitrate in animal studies [32-36], evidence in humans remains inconsistent [9]. As illustrated in Chapter 8, consumption of these compounds was not strongly associated with the risk of colorectal adenomas and colorectal cancer in the PLCO Screening Trial. To further elucidate these findings, we investigated the combined effect of these mutagens and genetic variants in genes involved in the activation and detoxification of these compounds (XME genes). Studying such gene-environment $(\mathrm{G} \times \mathrm{E})$ interactions may also help to identify individuals at highest risk of cancer based on both their meat consumption patterns and genetic risk profiles. This knowledge may eventually be used to tailor interventions aimed at cancer prevention. Although this is a promising avenue to pursue, the application of genetic based personalized nutrition in the immediate future is hampered the paucity of reproducible findings. This likely results from the limited power of many G×E interactions studies [37].

There are many approaches that can be used when studying GXE interactions as summarized by Hutter [38]. The strategy of choice depends on many factors including the study design and the gene and SNP selection strategy (ranging from agnostic genome wide comparisons to hypothesis driven studies). Within a nested case control setting we opted for a hypothesis based approach aimed at covering the complete genetic variation in the genes involved in meat mutagen metabolism pathways. Because the experimental literature on the role of XME genes in relation to HCA, PAH and NOC metabolism is far from complete, we did not restrict our analyses to those gene-mutagen interactions previously reported. We conducted a tagSNP selection (to maximize genetic coverage) and combined this with the selection of all SNPs with known or putative functional significance. This resulted in the selection of $>500$ SNPs that were studied in interaction with the five different meat mutagens. In order to reduce the multiple testing burden associated with this endeavor we applied Murcray's two-step approach [39], a novel data reduction technique, to increase statistical power. The essence of this approach is to screen on marginal environmental effects for the individual SNPs, and then test GXE interactions for these SNPs. Numerous comparable methods have been proposed in an attempt to improve the limited power of G×E interaction studies (as discussed in ref 40-41).

In line with our hypothesis, we found some evidence that common variants in XME genes may modify the association between meat mutagens and colorectal cancer. However, genetic variation in these genes could not explain as much of the (lack of) association between meat carcinogen intake and colorectal neoplasia as expected a priori. This is a common issue in many hypothesis driven GxE interaction studies. Despite many years of candidate gene studies in cancer, there are only a handful of examples of replicated and 
widely agreed-upon successes (e.g. ALDH2, alcohol and esophageal cancer [42]; NAT2, smoking and bladder cancer risk [43]). In our attempt to further elucidate the association between meat (carcinogens) and cancer risk we may need to shift our focus towards identifying novel loci by using agnostic GxE-wide interaction studies (GEWIS). Now that the costs of genotyping are rapidly declining, the first genome-wide initiatives to study GxE interactions have been reported in the literature (e.g. [44, 45]). However, for GEWIS to be a success, it is crucial to form consortia with standardized exposure data in order to increase statistical power.

All the previously discussed methods are single-marker approaches, studying one G×E interaction at a time. However, it is generally accepted that cancer results from a complex interplay of genetic and environmental risk factors. It may therefore be interesting to apply a more global approach that simultaneously considers al the genetic markers in an entire pathway of interest [46]. This may provide insight in how the carcinogenic effect of meat may vary according to a person's genetic profile.

This section illustrates that the study of GXE interactions is a rapidly developing discipline. As a consequence, many different designs, gene/SNP selection strategies, and methods of analysis are currently being developed and applied in an attempt to further decipher the diet related carcinogenesis process (i.e. meat consumption). The future will tell us what these new initiatives have brought us and how we can best proceed to move the nutritional cancer epidemiology field forward.

\section{Molecular pathological epidemiology, heme iron, and colorectal cancer}

It has been put forward that in contrast to HCA and PAH intake, the amount of heme iron consumption may be able to better explain the differential effect of red and white meat in colorectal carcinogenesis [47]. While experimental studies have generated considerable mechanistic evidence supportive of the role of heme iron in carcinogenesis, insight in the underlying mechanisms from population based observational studies are lacking. Colorectal cancer is, like many other cancers, a heterogeneous disease that can be characterized by different sets of (epi-)genetic alterations [19]. We therefore classified colorectal cancers into a limited number of groups based on their (specific point-) mutation status of a number of colorectal cancer key genes (i.e. APC, KRAS) and expression of P53. This enabled us to identify tumors that have arisen through common mechanisms and may thus reveal underlying mechanisms of carcinogenesis. As extensively discussed in Chapter 9, by applying this novel molecular pathological epidemiological approach we were able to show that heme iron was most likely associated with colorectal cancer through alkylating, rather than oxidative, DNA-damaging mechanisms (as evidenced by an increased risk of colorectal tumors with especially $\mathrm{G}>\mathrm{A}$ mutations).

Even though genetic aberrations in APC and KRAS are considered early events in colorectal carcinogenesis, it remains questionable whether the observed mutations are in fact caused by heme iron and have by itself driven cancer initiation or progression [19]. Heme iron exposure could also have created an environment that provided a selective advantage for clonal expansion of colonic cell with a specific molecular change [19]. Nevertheless, as outlined by Ogino et al., even if a given molecular change is consequential rather than causal, the change can be a good surrogate marker of a certain cancer pathway, but may 
also ultimately become a driver in later steps of tumor progression [19]. It is known that colorectal tumors often display considerable intratumor heterogeneity. We tried to capture some of this heterogeneity by isolating DNA from five separate $20 \mu \mathrm{m}$ sections of the tumor block [48], but may still have missed some relevant genetic tumor variation.

Comparable to the previously discussed studies of GxE interaction, molecular pathological epidemiology is also faced with multiple testing issues. The NLCS is one of the largest studies conducted to date that has collected information on molecular endpoints of (colorectal) cancer and baseline dietary intake (i.e. meat). However, despite this size, we were, like any other molecular pathological epidemiology study, faced with multiple exclusions based on the availability of tumor material and valid assay results [19]. In our effort to examine the association with heme iron, a subset analysis for different outcomes (KRAS mutated versus KRAS wild type tumors) was conducted. The sample size for rarer events (e.g. specific KRAS G>A or G>T mutations) may not have been large enough to provide adequate statistical power. However, because such specific point mutations (i.e. $G>A$ and $G>T$ ) are not limited to the genes under study, it may be interesting to sum the occurrence of these specific mutations in the entire tumor genome to increase power in future analyses using novel cancer genome sequencing techniques.

One solution to overcome the sample size problem is to pool data from independent studies that have both information on dietary exposure and molecular heterogeneity of the tumor available. Several large pooling initiatives are currently being developed. For example, in the Netherlands, the prestigious BBMRI rainbow initiative sets out to (further) enrich several prospective Dutch cohorts (including the NLCS) with tumor tissue material. From this, tissue microarrays will be constructed which will enable us to study protein expression of hundreds of tumors at the same time. When completed, this yields an extremely powerful infrastructure for molecular pathological epidemiology research into diet (i.e. meat) and cancer.

\section{RECOMMENDATIONS FOR FUTURE RESEARCH}

Based on the findings presented in this thesis, future research with respect to the study of (low) meat consumption, vegetarianism, and cancer risk may focus on the following:

- Further investigate the role of vegetarian and low meat diets on cancer risk, specifically focusing on cancer subtypes (by stage and location) and the explanatory role of specific components characteristic for these diets (e.g. by pooling of individual study data).

- As mentioned above, molecular epidemiology techniques (including GxE interactions, molecular pathological epidemiology) should be used to further elucidate the mechanisms underlying the meat cancer association. The field of molecular epidemiology is dynamic and new markers and associated statistical analysis techniques continue to be developed.

- The findings from observational molecular epidemiology should ultimately be tested in experimental studies to accurately assess the effect of specific dietary modifications (e.g. low meat diets) on intermediate biomarkers or cancer outcomes in a controlled setting. Such studies can help to design personalized 
cancer prevention strategies that may be more effective than the existing general guidelines (e.g. those from the WCRF [9]).

- Use the knowledge of specific meat-related carcinogenic pathways to identify strategies to change or intervene on the processes that lead to meat carcinogen formation such as changing the diet, the production process, or additives. Promising examples include adding calcium carbonate $[49,50]$ (to bind heme iron) or vitamin C or E $[49,51]$ (to inhibit nitrosamine formation) to the diet, developing anaerobic storage and packaging techniques [52] (to prevent oxidation), and omitting nitrite from curing solutions of processed meat products (e.g. by adding phytochemicals [53]).

\section{CONCLUDING REMARKS}

This thesis illustrates that meat consumption is a complex exposure variable that calls for multiple approaches when studying its association with cancer risk. We believe that the dietary pattern and meat carcinogen approach that were applied in this thesis have shown to provide a valuable addition to the previously more common conducted traditional analyses at the food level (i.e. (subtypes of) meat).

The epidemiological studies described in this thesis do not support a strong association between meat consumption and the major cancers under study. When compared to individuals consuming meat on a daily basis, our univariate analyses suggest that vegetarians and low meat consumers were at a reduced the risk of lung and colorectal cancer, respectively, but this effect attenuated and lost statistical significance after correction for confounders. Based on our cancer risk estimates, there is no reason to completely exclude meat from the diet. In fact, individuals consuming small amounts of meat may be less prone to develop vitamin B12 deficiencies as compared to strict vegetarians. When consuming meat very infrequently, it could be argued that it may be more important to make a sound decision concerning which type of meat to include in your diet. Our findings for example suggest that meat rich in heme iron likely increases the risk of colorectal cancer.

\section{REFERENCES}

1. Rothman K.J. and Greenland S., Modern Epidemiology. 1998, Philadelphia: Lippincott-Raven Publishers.

2. Goldbohm, R.A., P.A. van den Brandt, and E. Dorant, Estimation of the coverage of Dutch municipalities by cancer registries and PALGA based on hospital discharge data. Tijdschrift Soc Gezondheidsz, 1994. 72: p. 80-84.

3. Schouten, L.J., et al., Completeness of cancer registration in Limburg, The Netherlands. Int J Epidemiol, 1993. 22(3): p. 369-76.

4. Kristal, A.R., U. Peters, and J.D. Potter, Is it time to abandon the food frequency questionnaire? Cancer Epidemiol Biomarkers Prev, 2005. 14(12): p. 2826-8.

5. Willett, W.C., Nutritional Epidemiology. $3^{\text {rd }}$ ed. 2013, New York: Oxford University Press.

6. Goldbohm, R.A., et al., Reproducibility of a food frequency questionnaire and stability of dietary habits determined from five annually repeated measurements. Eur J Clin Nutr, 1995. 49(6): p. 420-9.

7. Willett, W.C., et al., Reproducibility and validity of a semiquantitative food frequency questionnaire. Am J Epidemiol, 1985. 122(1): p. 51-65.

8. Hartman, A.M., et al., Reproducibility of a self-administered diet history questionnaire administered three times over three different seasons. Nutr Cancer, 1996. 25(3): p. 305-15. 
9. World Cancer Research Fund/American Institute for Cancer Research, Food, Nutrition, Physical Activity, and the Prevention of Cancer: a Global Perspective. 2007, AICR: Washington DC.

10. www.fao.org.

11. Fraser, G.E., Vegetarian diets: what do we know of their effects on common chronic diseases? Am J Clin Nutr, 2009. 89(5): p. 1607S-1612S.

12. Haddad, E.H. and J.S. Tanzman, What do vegetarians in the United States eat? Am J Clin Nutr, 2003. 78(3 Suppl): p. 626S-632S.

13. Vinnari, M., et al., Identifying vegetarians and their food consumption according to self-identification and operationalized definition in Finland. Public Health Nutr, 2009. 12(4): p. 481-8.

14. Sinha, R. and N. Rothman, Exposure assessment of heterocyclic amines (HCAs) in epidemiologic studies. Mutat Res, 1997. 376(1-2): p. 195-202.

15. Sinha, R., An epidemiologic approach to studying heterocyclic amines. Mutat Res, 2002. 506-507: p. 197-204.

16. Sinha, R., et al., Development of a food frequency questionnaire module and databases for compounds in cooked and processed meats. Mol Nutr Food Res, 2005. 49(7): p. 648-55.

17. Sinha, R., et al., Heterocyclic amine content of pork products cooked by different methods and to varying degrees of doneness. Food Chem Toxicol, 1998. 36(4): p. 289-97.

18. Cross, A.J., et al., Developing a heme iron database for meats according to meat type, cooking method and doneness level. Food Nutr Sci, 2012. 3(7): p. 905-913.

19. Ogino, S., et al., Molecular pathological epidemiology of colorectal neoplasia: an emerging transdisciplinary and interdisciplinary field. Gut, 2011. 60(3): p. 397-411.

20. Wild, C., P. Vineis, and S. Garte, Molecular Epidemiology of Chronic Disease. 2008, West Sussex, England: John Wiley \& Soncs Ltd.

21. Fraser, G.E., Associations between diet and cancer, ischemic heart disease, and all-cause mortality in non-Hispanic white California Seventh-day Adventists. Am J Clin Nutr, 1999. 70(3 Suppl): p. 532S-538S.

22. Phillips, R.L., Role of life-style and dietary habits in risk of cancer among seventh-day adventists. Cancer research, 1975. 35(11 Pt. 2): p. 3513-22.

23. Vandenbroucke, J.P., Observational research, randomised trials, and two views of medical science. PLoS Med, 2008. 5(3): p. e67.

24. Cade, J.E., et al., Common dietary patterns and risk of breast cancer: analysis from the United Kingdom Women's Cohort Study. Nutr Cancer, 2010. 62(3): p. 300-6.

25. Key, T.J., et al., Cancer incidence in British vegetarians. Br J Cancer, 2009. 101(1): p. 192-7.

26. Key, T.J., et al., Cancer incidence in vegetarians: results from the European Prospective Investigation into Cancer and Nutrition (EPIC-Oxford). Am J Clin Nutr, 2009. 89(5): p. 1620S-1626S.

27. Sanjoaquin, M.A., et al., Nutrition, lifestyle and colorectal cancer incidence: a prospective investigation of 10998 vegetarians and non-vegetarians in the United Kingdom. Br J Cancer, 2004. 90(1): p. 118-21.

28. Hu, F.B., Dietary pattern analysis: a new direction in nutritional epidemiology. Curr Opin Lipidol, 2002. 13(1): p. 3-9.

29. Blank, M.M., et al., Dietary fat intake and risk of ovarian cancer in the NIH-AARP Diet and Health Study. Br J Cancer, 2012. 106(3): p. 596-602.

30. Prentice, R.L., et al., Low-fat dietary pattern and cancer incidence in the Women's Health Initiative Dietary Modification Randomized Controlled Trial. J Natl Cancer Inst, 2007. 99(20): p. 1534-43.

31. Wallin, A., N. Orsini, and A. Wolk, Red and processed meat consumption and risk of ovarian cancer: a dose-response meta-analysis of prospective studies. Br J Cancer, 2011. 104(7): p. 1196-201.

32. Bogovski, P. and S. Bogovski, Animal Species in which N-nitroso compounds induce cancer. Int J Cancer, 1981. 27(4): p. 471-4.

33. Ito, N., et al., A new colon and mammary carcinogen in cooked food, 2-amino-1-methyl-6phenylimidazo[4,5-b]pyridine (PhIP). Carcinogenesis, 1991. 12(8): p. 1503-6.

34. Ochiai, M., et al., Induction of intestinal tumors and lymphomas in C57BL/6N mice by a food-borne carcinogen, 2-amino-1-methyl-6-phenylimidazo[4,5-b]pyridine. Jpn J Cancer Res, 2002. 93(5): p. 47883.

35. Ohgaki, H., S. Takayama, and T. Sugimura, Carcinogenicities of heterocyclic amines in cooked food. Mutat Res, 1991. 259(3-4): p. 399-410.

36. Sugimura, T., Overview of carcinogenic heterocyclic amines. Mutat Res, 1997. 376(1-2): p. 211-9. 
37. Murcray, C.E., et al., Sample size requirements to detect gene-environment interactions in genomewide association studies. Genet Epidemiol, 2011. 35(3): p. 201-10.

38. Hutter, C.M., et al., Gene-environment interactions in cancer epidemiology: a National Cancer Institute Think Tank report. Genet Epidemiol, 2013. 37(7): p. 643-57.

39. Murcray, C.E., J.P. Lewinger, and W.J. Gauderman, Gene-environment interaction in genome-wide association studies. Am J Epidemiol, 2009. 169(2): p. 219-26.

40. Aschard, H., et al., Challenges and opportunities in genome-wide environmental interaction (GWEI) studies. Hum Genet, 2012. 131(10): p. 1591-613.

41. Hsu, L., et al., Powerful cocktail methods for detecting genome-wide gene-environment interaction. Genet Epidemiol, 2012. 36(3): p. 183-94.

42. Brooks, P.J., et al., The alcohol flushing response: an unrecognized risk factor for esophageal cancer from alcohol consumption. PLoS Med, 2009. 6(3): p. e50.

43. Garcia-Closas, M., et al., Common genetic polymorphisms modify the effect of smoking on absolute risk of bladder cancer. Cancer Res, 2013. 73(7): p. 2211-20.

44. Siegert, S., et al., Genome-wide investigation of gene-environment interactions in colorectal cancer. Hum Genet, 2013. 132(2): p. 219-31.

45. Wu, C., et al., Genome-wide association analyses of esophageal squamous cell carcinoma in Chinese identify multiple susceptibility loci and gene-environment interactions. Nat Genet, 2012. 44(10): p. 1090-7.

46. Yu, K., et al., A flexible Bayesian model for studying gene-environment interaction. PLoS Genet, 2012. 8(1): p. e1002482.

47. Bastide, N.M., F.H. Pierre, and D.E. Corpet, Heme iron from meat and risk of colorectal cancer: a metaanalysis and a review of the mechanisms involved. Cancer Prev Res (Phila), 2011. 4(2): p. 177-84.

48. Brink, M., et al., K-ras oncogene mutations in sporadic colorectal cancer in The Netherlands Cohort Study. Carcinogenesis, 2003. 24(4): p. 703-10.

49. Pierre, F.H., et al., Calcium and alpha-tocopherol suppress cured-meat promotion of chemically induced colon carcinogenesis in rats and reduce associated biomarkers in human volunteers. Am J Clin Nutr, 2013. 98(5): p. 1255-62.

50. Allam, O., et al., Calcium carbonate suppresses haem toxicity markers without calcium phosphate side effects on colon carcinogenesis. Br J Nutr, 2011. 105(3): p. 384-92.

51. Lunn, J.C., et al., The effect of haem in red and processed meat on the endogenous formation of $\mathrm{N}$ nitroso compounds in the upper gastrointestinal tract. Carcinogenesis, 2007. 28(3): p. 685-90.

52. Santarelli, R.L., et al., Meat processing and colon carcinogenesis: cooked, nitrite-treated, and oxidized high-heme cured meat promotes mucin-depleted foci in rats. Cancer Prev Res (Phila), 2010. 3(7): p. 852-64.

53. www.phytome.eu. 

S

Summary / Samenvatting 


\section{ENGLISH SUMMARY}

Since the early ecological evidence in the seventies of the last century suggested that meat consumption was associated with the risk of (especially colorectal) cancer, hundreds of individual-level epidemiological studies have reported on the relation between meat and cancer. However, despite these scientific efforts, the effect of meat consumption on cancer risk remains a controversial issue.

For this reason, this thesis set out to investigate the association between meat consumption and cancer risk using a multi-dimensional epidemiological approach. The first chapters of this thesis focus on vegetarian and low meat dietary patterns in relation to cancer risk, whereas specific mechanisms of meat-related carcinogenesis (i.e. dietary fat, heterocyclic amines (HCAs), polycyclic aromatic hydrocarbons (HCAs), nitrite/nitrate, and heme iron) are examined in the second part of this thesis.

\section{PART A: A Dietary Pattern Approach}

This section starts with two studies on meat consumption patterns and non-cancer health outcomes because these may, in turn, affect the association between low meat diets and cancer risk.

It is well-accepted that a large body size is associated with an increased risk of many cancers, but the association between meat consumption and prospective weight gain remains to be elucidated. In Chapter $\mathbf{2}$, we assessed the association between meat consumption at baseline and change in BMI over six and $14 \mathrm{y}$ of follow-up using data from the population based Netherlands Cohort Study (NLCS). At baseline, all participants filled out a questionnaire on dietary habits, anthropometry, and other risk factors for cancer. The NLCS is a case-cohort design with a subcohort of 5000 individuals randomly selected from the larger cohort on recruitment into the study. Follow-up of the subcohort has allowed for the additional accumulation of prospective data regarding risk factors for cancer including body weight. This subcohort was the study population used in this chapter. We showed that total meat consumption at baseline was positively associated with BMI at baseline, but it did not strongly affect subsequent weight gain over a 14 y period. Nonetheless, modest differential BMI change effects were observed for some subtypes of meat. Results remained similar when stratifying on median baseline BMI.

Individuals following a vegetarian or vegan diet are likely at higher risk of developing nutrient deficiencies i.e. vitamin B12. This vitamin is naturally present only in foods of animal origin. In Chapter $\mathbf{3}$ of this thesis we therefore investigated whether vegans and strict vegetarians were at an increased risk of developing a deficiency. We used crosssectional data from the large European Prospective Investigation into Cancer and Nutrition Oxford cohort to show that mean serum vitamin B12 was highest among omnivores $(n=226)$, intermediate in vegetarians $(n=231)$, and lowest in vegans $(n=232)$. Nearly half of the vegans were categorized as vitamin B12 deficient and would be expected to have a higher risk of developing clinical symptoms related to vitamin B12 deficiency. There was no significant association between age or duration of adherence to a vegetarian or a vegan diet and vitamin B12. Based on these findings, both vegetarians and vegans should ensure a regular intake of sufficient vitamin B12 from fortified foods and/or supplements. 
The few prospective cohort studies that were specifically set out to study cancer incidence in vegetarians have reported mixed results. In addition, no study has examined the effects of low meat consumption on cancer risk. In order to study how a vegetarian and low meat diet (i.e. meat 1 day/week) influences the risk of cancer compared to a more common high meat diet, we extracted the NLCS-Meat Investigation Cohort (NLCS-MIC) from the total NLCS cohort. The NLCS was followed up for cancer incidence through linkage to the Netherlands Cancer Registry, and the Netherlands Pathology Registry. The NLCS aimed to over-represent vegetarians and low meat consumers at recruitment by intentionally contacting them through health food shops and magazines. By using the extensive dietary data from the $F F Q$, we were able to classify vegetarians based on self-reporting (1 question) as well as on the more accurate and extensive FFQ data. As a result, the NLCSMIC was defined by combining all FFQ-confirmed vegetarians $(n=702)$, pescetarians $(n=394)$ and 1 day/week meat consumers $(n=1396)$ from the total NLCS cohort, with a random sample of 2-5 days/week and 6-7 days/week meat consumers ( $n=2965$ and 5648, respectively). Consequently, we could not only study vegetarianism and low meat consumption in relation to major cancer outcomes (colorectal, lung, female breast and prostate) but were also able to investigate the reliability of self-defined vegetarianism.

In line with our expectations, a baseline comparison of the NLCS-MIC (Chapter 4) indicated that vegetarians, pescetarians, and individuals consuming meat for 1 day/week had a more favorable distribution of (non-)dietary cancer risk factors (e.g. high intakes of fruit and vegetables, lower smoking rates) compared to regular meat consumers (defined as individuals who eat meat for 6-7 days/week). Additionally, we observed that as much as $50 \%$ of all the individuals who classified themselves as vegetarian (based on 1 item on the first page of the questionnaire) reported (some) meat or fish consumption on the extensive FFQ. However, the misclassification that occurred in terms of baseline diet (other than meat) and lifestyle characteristics using these self-reports was relatively small. We showed that the proportion of prevalent cancer cases was highest among the vegetarians and pescetarians and significantly decreased with increasing meat intake. This suggests that such selection mechanisms should be taken into consideration in etiologic studies.

We then investigated the association between these meat consumption groups and colorectal cancer risk in Chapter 5 in the NCLS-MIC. After 20.3 years of follow-up and exclusion of prevalent cancer cases, 437 colorectal cancer cases (307 colon, 92 rectal) were available for analyses. Compared to high meat consumers, both non- and low meat consumers had a non-significantly decreased risk of colorectal, especially rectal, cancer which attenuated after adjustment for confounders. In fact, most of the differences in risk estimates between these meat consumption groups could be explained by differences in dietary pattern other than meat intake. No statistically significant differences in colorectal cancer risk between vegetarians and non-vegetarians were observed, but the inverse association was stronger using FFQ-confirmed vegetarian status over self-defined status.

Chapter 6 describes the association between meat consumption group and the risk of lung, female breast and advanced and overall prostate cancer using data from the NLCS-MIC after 20.3 y of follow-up. In age- and sex adjusted analyses, a statistically significant reduced risk of lung cancer for vegetarians and pescetarians was found when compared with 6-7 days/week meat consumers (Hazard Ration (HR): $0.44,95 \%$ confidence interval 
(CI): 0.21-0.94, and HR: $0.28,95 \% \mathrm{Cl}: 0.09-0.88$ respectively). However, further adjustment for confounding (especially smoking) attenuated these associations such that they were no longer significant (HR: 0.85 and 0.54, respectively). Vegetarians, pescetarians and 1 day/week meat consumers did not have a reduced risk female breast and overall prostate cancer compared to individuals consuming meat on a daily basis. Our unexpected observation that $\leq 1$ day/week meat consumption was associated with increased risk of advanced prostate cancer warrants investigation in other studies.

\section{PART B: A Mechanistic Approach}

In the second part of this thesis, we set out to study each of the proposed mechanisms of meat-related carcinogenicity in relation to the cancer endpoints most likely to be affected.

Dietary fat, particularly saturated fat, has long been suspected to be responsible for the meat-cancer association. However, epidemiological studies have been inconsistent and intervention studies aimed at lowering total fat intake failed to confirm any association with cancer risk except for cancer of the ovaries. By performing a case-cohort analysis in the total NLCS cohort, we did not find evidence that fat from fresh and processed meats increased the risk of ovarian cancer after 16.3 y of follow-up (Chapter 7). Neither did we find a clear dose response association for animal fat, saturated fat, or total fat which may be present in high quantities in a meat-rich diet. The multi-variable HR for the highest compared to the lowest quintile of trans-unsaturated fatty acid intake was $1.51(95 \% \mathrm{Cl}$ : 1.04-2.20; P-trend=0.01). This type of fat, however, does not stem from meat, but from margarines, packaged baked products and fast foods.

Specific meat mutagens, including HCAs, PAHs, and N-nitroso compounds (NOCs), may be involved in colorectal carcinogenesis depending on their activation or detoxification by phase I and II xenobiotic metabolizing enzymes (XME). Because large scale studies are lacking we wanted to investigate the interplay between five meat mutagens and genetic variation in $18 \mathrm{XME}$ genes in relation to advanced colorectal adenoma, and colorectal cancer (Chapter 8). To this extent we performed two nested case control studies in the Prostate, Lung, Colorectal, and Ovarian Cancer (PLCO) Screening Trial. Dietary intake of meat mutagens was assessed using a food frequency questionnaire (FFQ) with a detailed meat-cooking module. After adjusting for multiple testing we observed one interaction between HCA 2-amino-3,8-dimethylimidazo[4,5-f]quinoxaline (MelQx) intake and a NAT1 polymorphism in the adenoma study. Despite that this was the largest and most comprehensive study to date, genetic variation in XME genes could not explain as much of the association between meat carcinogen intake and colorectal neoplasia as expected a priori. This is a common issue in many comparable hypothesis driven $\mathrm{G} \times \mathrm{E}$ interaction studies. However, the study of GxE interactions is a rapidly developing discipline and much effort is being put into developing new study designs, gene/SNP selection strategies, and methods of analysis to increase statistical power.

It has been suggested that the positive association between red meat consumption and colorectal cancer can be explained by the high heme iron content of red meat. Because heme iron has shown to promote specific mutations, we set out to link heme iron data to colorectal cancer with specific mutations in key-genes. For this study, which is described in Chapter 9 we used data from the total NLCS cohort. After 7.3 y of follow-up, tumor tissue 
samples were collected for nearly all colorectal cancer cases based on which KRAS and APC mutation status and P53 expression status could be determined. The results from this research support the hypothesis that heme iron increases the risk of colorectal cancer. Heme iron intake was associated with an increased risk of colorectal tumors harboring $G>A$ transitions in KRAS and APC and overexpression of P53. No associations were found with CRC harboring G>T mutations in KRAS or APC. These novel findings suggest that alkylating rather than oxidative DNA damaging mechanisms are involved in heme-induced colorectal carcinogenesis. In addition, it illustrates that novel molecular pathological epidemiology techniques can lead to a better understanding of the underlying pathways.

This thesis concludes with a summary of the main findings, a discussion about the limitations, and implications for future research (Chapter 10). We illustrate that meat consumption is a complex exposure variable that calls for multiple approaches when studying its association with cancer risk. Overall, our results suggest do not support a strong association between meat consumption and the major cancers under study. When compared to individuals consuming meat on a daily basis, our univariate analyses suggest that vegetarians and low meat consumers were at a reduced the risk of lung and colorectal cancer, respectively, but this effect attenuated and lost statistical significance after correction for confounders. We paid specific interest to issues relating to confounding when studying the health effects of vegetarian and low meat diets since we showed that these populations exhibit a number of other potential cancer preventive factors and behaviors next to the (near) avoidance of meat. Our observation that heme iron may promote colorectal cancer risk through specific alkylating mechanisms provides important new mechanistic evidence that red meat consumption may indeed be a risk factor for colorectal cancer. 


\section{NEDERLANDSE SAMENVATTING}

In de jaren zeventig van de vorige eeuw suggereerde ecologisch onderzoek dat een hoge vleesconsumptie mogelijk geassocieerd was met een hoger risico op (met name darm-) kanker. Sindsdien zijn er honderden epidemiologische studies op het individuele niveau uitgevoerd om de relatie tussen vlees en kanker beter te kwantificeren. Ondanks deze wetenschappelijke inspanningen blijft de relatie tussen vleesconsumptie en het risico op kanker controversieel.

Om deze reden heeft dit proefschrift als doel de associatie tussen vleesconsumptie en het kankerrisico verder te onderzoeken. Hiervoor is gebruik gemaakt van een epidemiologische benadering vanuit een tweetal perspectieven. In de eerste hoofdstukken gaat de aandacht uit naar de relatie tussen vegetarische en laag vlees voedingspatronen en het risico op kanker. In het tweede deel van dit proefschrift is gekeken naar specifieke mechanismes waarop vlees het risico of kanker kan beïnvloeden (de hoeveelheid vet, heterocyclische amines (HCAs), polyaromatische koolwaterstoffen (PAKs), nitraat/nitriet, en heemijzer).

\section{DEEL A: Een voedingspatroon benadering}

Deze sectie start met twee studies naar de relatie tussen vleesconsumptie en niet-kanker gerelateerde gezondheidseffecten zoals gewichtstoename en vitamine B12 deficiënties. Deze gezondheidseffecten kunnen namelijk op hun beurt de relatie tussen vleesconsumptie en kanker mogelijk beïnvloeden.

Alhoewel over het algemeen wordt aangenomen dat een hoog lichaamsgewicht het risico op veel verschillende type kankers verhoogd, is er nog maar weinig bekend over de relatie tussen vleesconsumptie en gewichtstoename over de tijd. In hoofdstuk 2, hebben we bekeken hoe vleesconsumptie (gemeten aan de start van het onderzoek) gerelateerd is aan veranderingen in de Body Mass Index (BMI in $\mathrm{kg} / \mathrm{m}^{2}$ ) na 14 jaar follow-up. Dit onderzoek is uitgevoerd binnen de Nederlandse Cohort Studie naar voeding en kanker (NLCS). Bij aanvang van de studie in 1986 hebben alle 120.852 deelnemers een vragenlijst ingevuld over eetgewoonte (middels een voedselfrequentievragenlijst (FFQ)), antropometrie, en andere risicofactoren voor kanker. De NLCS hanteert een case-cohort benadering wat inhoudt dat bij aanvang van het onderzoek, na de beginmeting, een subcohort van 5000 personen willekeurig is geselecteerd. Van dit subcohort is na zes en 14 jaar follow-up extra informatie verzameld over lichaamsgewicht. Met behulp van deze gegevens hebben wij aangetoond dat de totale vleesconsumptie bij aanvang van de studie in 1986 sterk positief geassocieerd was met de BMI op dat zelfde tijdstip. De totale vleesconsumptie heeft echter geen sterke invloed op gewichtstoename over de daaropvolgende periode van 14 jaar. Desalniettemin vonden wij enkele bescheiden indicaties dat sommige subtypes van vlees een verschillend effect op de verandering in BMI hebben.

Personen die een vegetarisch of veganistisch dieet volgen hebben mogelijk een grotere kans op het ontwikkelen van voedingsdeficiënties zoals een vitamine B12 tekort. Deze vitamine is alleen aanwezig in voedsel van dierlijke oorsprong. In hoofdstuk $\mathbf{3}$ van dit proefschrift hebben we daarom gekeken of vegetariërs en veganisten een verhoogd risico hebben op een dergelijk tekort. We hebben daarvoor gebruik gemaakt van cross-sectionele data van het grote 'European Prospective Investigation into Cancer and Nutrition (EPIC) 
Oxford' cohort. Daarin hebben we aangetoond dat serum vitamine B12 waardes het hoogste waren in vleeseters $(n=226)$, gemiddelde waardes hadden in vegetariërs $(n=231)$ en het laagste waren in veganisten (232). Bijna de helft van alle veganisten werd geclassificeerd als vitamine B12 deficiënt en heeft naar verwachting een groter risico op het ontwikkelen van klinische symptomen die gerelateerd zijn aan een vitamine B12 deficiëntie. Wij vonden geen significante relatie tussen de leeftijd, duur van het vegetarische of veganistisch dieet en bloed waardes van vitamine B12. Gebaseerd op deze bevindingen zouden zowel vegetariërs en veganisten er voor moeten zorgen voldoende vitamine B12 uit verrijkte voeding en/of voedingssupplementen binnen te krijgen.

Het beperkte aantal prospectieve cohort studies dat speciaal is opgezet om naar kankerincidentie in vegetariërs te kijken heeft tot dusver gemengde resultaten opgeleverd. Daarnaast heeft geen enkele studie gekeken wat de relatie is tussen een lage vlees consumptie (gedefinieerd als één dag per week vlees) en het risico op kanker. Om te onderzoeken hoe een vegetarische en een laag vlees dieet het risico op kanker beïnvloedt in vergelijking met een meer gebruikelijk hoog vlees dieet, hebben wij het NLCS-Meat Investigation Cohort (NLCS-MIC) geëxtraheerd uit het totale NLCS cohort. Het NLCS cohort is opgevolgd om nieuwe kanker patiënten over de tijd te kunnen identificeren. Bij de opzet van het onderzoek is getracht het aantal vegetariërs en mensen met een lage vleesinname te vergroten door deze doelgroep te benaderen via gezondheidsbladen en reformwinkels. Door de uitgebreide dataverzameling, waren wij in staat om vegetariërs zowel te classificeren op basis van zelfrapportage (één vraag) en op basis van meer gedetailleerde FFQ. NLCS-MIC is uiteindelijke gecreëerd door alle 'FFQ-bevestigde'- vegetariërs ( $n=702)$, pescetariërs (oftewel viseters) ( $n=394)$, en 1 dag/week vleeseters $(n=1396)$ uit het totale NLCS-cohort te combineren met een random sample van personen die 2-5 dagen/week en 6-7 dagen/week vlees eten (respectievelijk $n=2965$ en 5648). Als direct gevolg hiervan konden wij niet alleen onderzoeken hoe een vegetarisch- en laag vlees dieet het risico op kanker beïnvloedt, maar ook hoe betrouwbaar de zelfrapportage van de vegetarische leefstijl is.

In de lijn der verwachting toonde een baseline vergelijking van het NLCS-MIC cohort in hoofdstuk 4 aan dat vegetariërs, pescetariers, en personen die slechts 1 dag/week vlees eten een gunstiger profiel van kankerrisicofactoren hadden (bijvoorbeeld een hogere inname van groente en fruit, lagere prevalentie van roken) in vergelijking met personen die (bijna) dagelijks vlees aten. Daarnaast vonden wij dat $50 \%$ van de personen die zichzelf als vegetariër classificeert toch aangeeft vis en/of vlees te eten op de uitgebreide FFQ. De misclassificatie die optreedt in termen van baseline voedselinname en leefstijlfactoren wanneer men gebruik maakt van zelfrapportage is echter relatief klein. Daarnaast zagen wij dat de proportie personen die op de baseline vragenlijst rapporteerde een kanker te hebben (gehad) het grootste was onder de vegetariërs en pescerariërs. Deze proportie nam verder significant af naar mate men meer vlees rapporteert te eten. Dit suggereert dat in etiologisch onderzoek rekening dient te moeten worden gehouden met dergelijke onderliggende bias.

Vervolgens onderzochten we in hoofdstuk 5 de associatie tussen deze vleesconsumptie groepen en het risico op colorectaal kanker in NLCS-MIC. Na 20.3 jaar follow-up, en exclusie van deelnemers die rapporteerde kanker te hebben (gehad) op de baseline vragenlijst, 
bleven er 437 colorectaalkanker patiënten (307 colon, 92 rectaal) over voor de analyses. Zowel vegetariërs als laag vlees eters hadden een niet significant verlaagd risico op op colorectaal, voornamelijk rectaal, kanker in vergelijking met personen die veel vlees eten. Dit effect werd minder sterk wanneer er voor confounders werd gecorrigeerd. De meeste verschillen in risicoschattingen tussen de vleesconsumptie groepen kon worden verklaard door verschillen in voedselconsumptie anders dan vleesinname. Wij observeerde geen statistisch significante verschillen in colorectaalkanker risico tussen vegetariërs en nietvegetariërs. De inverse associaties waren echter wel sterker wanneer we voor de classificatie van vegetariërs gebruik maakten van de FFQ data in plaats van zelfrapportage.

Hoofdstuk 6 beschrijft de relatie tussen vleesconsumptie groep en het risico op longkanker, borstkanker (alleen vrouwen), en gevorderd- en totaal prostaatkanker. Voor deze analyses werd wederom gebruik gemaakt van gegevens van het NLCS-MIC cohort na 20.3 jaar follow-up. In de univariate analyses hadden vegetariers een meer dan twee keer zo laag statistisch significant risico op longkanker in vergelijking met personen die 6-7 dagen per week vlees eten. Voor de groep pescetariërs was dit risico 3.5 keer zo laag. Wanneer we echter ook corrigeerde voor confounding (voornamelijk roken) attenueerde deze risicoschattingen en waren deze niet meer significant. Vegetariërs, pescetariërs en 1 dag/week vlees eters hadden geen verlaagd risico op borstkanker en totaal prostaatkanker in vergelijking met personen die dagelijks vlees eten. Wij zijn de eerste die rapporteren over de relatie tussen vleesconsumptie groep en gevorderd prostaatkanker risico. De onverwachte observatie dat mannen die $\leq 1 \mathrm{dag} /$ week vlees aten een verhoogd risico op gevorderd prostaatkanker hebben vraagt om verder onderzoek in andere studies.

\section{DEEL B: Een mechanistische benadering}

Het tweede deel van dit proefschrift had als doel te onderzoeken via welke mechanismes vlees mogelijk het risico op kanker verhoogd. Hierbij hebben wij enkele specifieke vleescarcinogenen onderzocht in relatie tot het kanker eindpunt waar deze het meest waarschijnlijk mee is geassocieerd.

Het is lang gesuggereerd dat voedingsvet, en voornamelijk verzadigd vet, verantwoordelijk zou zijn voor de relatie tussen vleesconsumptie en het risico op kanker. Epidemiologische studies hebben echter inconsistente resultaten opgeleverd en interventiestudies gericht op het verlagen van de vetinname hebben ook geen risicoverlagende effect aangetoond, behalve voor kanker aan de eierstokken. In hoofdstuk 7 beschrijven wij een case-cohort analyse in het totale NLCS-cohort waarbinnen wij geen bewijs hebben gevonden dat vet uit vers vlees en/of vleeswaren het risico op eierstokkanker na 16.3 jaar follow-up beinvloed. Wij vonden ook geen duidelijke dosis effect relatie voor dierlijk vet, verzadigd vet en totaal vet, welke allen in grote mate aanwezig kunnen zijn een een vleesrijk voedingspatroon. Vrouwen met de hoogste inname van transonverzadigde vetzuren hadden een $50 \%$ statistisch significant hoger risico op ovariumkanker dan vrouwen met de laagste inname na correctie voor confounders. Dit type vet is echter niet afkomstig uit vlees maar uit margarines en fastfood.

Specifieke vleescarcinogenen, zoals de heterocyclische amines (HCAs), polyaromatische koolwaterstoffen (PAKs), en $\mathrm{N}$-nitroso verbindingen (NOCs), zijn mogelijk betrokken in het ontstaan van darmkanker. De kankerverwekkende werking van deze componenten is 
afhankelijk van hun activatie en detoxificatie door fase I en II xenobiotica-metaboliserende enzymen (XME). Hier is echter nog weinig onderzoek naar gedaan in grootschalige epidemiologische studies. Om deze reden wilden wij in hoofdstuk $\mathbf{8}$ onderzoeken wat de gecombineerde effecten van de bovengenoemde vleescarcinogenen en genetische varianten in $18 \mathrm{XME}$ genen zijn in relatie tot het risico op poliepen in de dikke darm en dikke darmkanker. Hiertoe zijn twee geneste patiëntcontrole onderzoeken (één bij patiënten met poliepen en één bij patiënten met colorectaal kanker) uitgevoerd binnen de "Prostate, Lung, Colorectal, and Ovarian Cancer (PLCO) Screening Trial". Voedingsinname van de vleescarcinogenen was bepaald doormiddel van een FFQ met een gedetailleerde vleesbereidingsmodule. Na correctie voor multiple testing vonden wij één interactie tussen de HCA 2-amino-3,8-dimethylimidazo[4,5-f]quinoxaline (MelQx) en een polymorfisme in het NAT1 gen in de poliepstudie. Ondanks dat dit de grootste en meest uitgebreide studie was die tot dusver was uitgevoerd, kon genetische variatie in XME genen slechts in beperpkte mate de associatie tussen vleescarcinogeen inname en colorectale neoplasie verklaren.

Het is gesuggereerd dat het positieve verband tussen rood vlees consumptie en het risico op colorectaal kanker kan worden verklaard door de hoge concentratie heem ijzer in dit type vlees. Omdat experimenteel onderzoek heeft aangetoond dat blootstelling aan heemijzer specifieke mutaties in het DNA kan veroorzaken, hebben wij onderzocht of heemijzer inname geassocieerd was met specifieke mutaties in darmkankergenen. Voor deze studie, welke beschreven is in hoofdstuk $\mathbf{9}$, hebben wij gebruik gemaakt van gegevens van het totale NLCS cohort. Na 7.3 jaar follow-up is DNA geïsoleerd uit de tumor van bijna alle darmkankerpatiënten. In dit tumor DNA is zowel de mutatie status van het KRAS en $A P C$ gen, en de expressie van P53 bepaald. De resultaten van dit onderzoek bekrachtigen de hypothese dat heemijzer het risico op colorectaalkanker verhoogt. Heemijzer was geassocieerd met een verhoogd risico op colorectaalkanker gekenmerkt door G-naar-A mutaties in KRAS en APC en overexpressie van P53. Er werd geen verband gevonden tussen heemijzer en het risico op darmkanker met G-naar-T mutaties in KRAS en APC. Deze nieuwe bevindingen suggereren dat het kanker verhogende effect van heemijzer mogelijk wordt veroorzaakt door alkylatie en niet door andere mechanismes.

Dit proefschrift eindigt met een samenvatting van de belangrijkste bevindingen, een discussie over de limitaties en implicaties voor toekomstig onderzoek (hoofdstuk 10). Vleesconsumptie is een complexe leefstijl welke vanuit verschillende invalshoeken dient te worden onderzocht wanneer men het risico tussen vlees en kanker wil bestuderen. Onze resultaten laten geen sterk verband zien tussen vleesconsumptie en het risico op de onderzochte kankers. Uit onze univariate analyses blijkt dat in vergelijking tot personen die dagelijks vlees eten, vegetariërs en laag vleeseters een lager risico hebben op respectievelijk long- en colorectaalkanker. Echter deze resultaten verzwakken en zijn niet langer statistisch significant wanneer we corrigeren voor confounders. In ons onderzoek hebben wij speciale aandacht besteed aan het begrip confounding omdat vegetariërs en laag vleeseters ook vaak een ander leefstijl en voedingspatroon hebben, buiten het (niet) eten van vlees als zodanig. Deze confounders kunnen op hen beurt ook het risico op kanker kan beinvloeden. Onze observatie dat heemijzer mogelijk het risico op colorectaalkanker vergroot door specifieke alkylerende mechanismen, verschaft belangrijk nieuwe mechanistisch bewijs dat rood vlees inderdaad een risicofactor is voor colorectaalkanker. 

Valorisation 
In addition to the scientific value of this thesis described in Chapters 2-10, the results of the research presented also has societal and economic value (either directly or following additional research). The challenge of bridging our research results with clinical care, public policy, and community action can best be met through knowledge transfer and exchange. This thesis applied a multi-dimensional approach to study the association between meat and cancer risk and hence included studies on different health outcomes and exposures. Because examples of value creation differ by discipline and type of research, the studies described in this thesis were grouped into three categories and the valorization possibilities for each section are described separately below.

\section{MEAT CONSUMPTION AND WEIGHT CHANGE}

Overweight and obesity have reached pandemic proportions resulting in a growing global burden of obesity related chronic disease [1]. At a population level, even moderate elevations in BMI and body weight over a long period of time have been shown to increase disease risk [2-4]. It is thus of great importance to determine effective and safe mechanisms for maintaining a healthy body weight; this can reduce the enormous health care costs associated with obesity related chronic disease and will greatly increase the quality of life of the individual.

Because several hypotheses suggested a role for meat consumption in weight management we set out to study if meat consumption was associated with weight gain in an elderly population (55-69 y) (Chapter 2). We observed only a small increase in BMI from baseline over a 14 y prospective follow-up. In line with these findings we did not find strong evidence for an association between (subtypes of) meat intake and prospective weight change. Other research indicates that men and women predominantly gain weight until middle age $[5,6]$. We also conducted retrospective and cross-sectional analyses that indeed suggest that meat consumption is associated with weight change over the first $40 \mathrm{y}$ of adult life. This offers a large window of opportunity for developing and implementing effective weight management strategies. Future studies may therefore focus on verifying and further deciphering these observations in observational and experimental settings. Such studies can provide important evidence for developing evidence-based dietary weight management guidelines that may be more effective than the existing recommendations.

\section{MEAT CONSUMPTION AND VITAMIN B12 DEFICIENCY}

The key message of the research described in Chapter 3 is that the vitamin B12 content of vegetarian (including vegan) diets is frequently below the reference limit. The vegetarian population, clinicians and policymakers are the principal target audience for this message and should be engaged to become active to take action. For example, more intense monitoring, fortification and supplementation of vitamin B12 will help to prevent negative vitamin B12 related health effects in persons adhering to these diets.

First and foremost, changing awareness and educating the vegetarian population to take effective vitamin B12 supplements is key in preventing nutritional deficiencies in this group. Although there are many active online vegetarian communities to date, by far not all of them are preaching scientifically valid dietary recommendations and advice. It is therefore important to use effective platforms to actively communicate our research results to this target population. The results of the paper described in Chapter 3 have featured in the 
September 2010 issue of the International Vegetarian Union newsletter and reached a wide target audience. This has prompted plenty of discussion on the importance of ensuring a reliable dietary source of vitamin B12 among the vegetarian community.

Early detection and quick treatment of a vitamin B12 deficiency are essential to prevent development of irreversible neurologic damage [7]. Making an accurate and timely diagnosis, however, can be challenging. The list of related signs and symptoms is long, varied, and non-specific and vitamin B12 deficiency is often misdiagnosed for some other condition $^{7}$. Increased awareness of this issue among clinicians may help to ensure a timely diagnosis (e.g. by inquiring after a person's diet when patients present with assymtomatic complaints).

The abovementioned valorization opportunities to prevent vitamin B12 deficiencies are hampered by the recommendation of the Health Council of the Netherlands to fortify bread and breakfasting cereals with folic acid [8]. Even though folic acid prevents neural type defects, high intakes can mask a vitamin B12 deficiency hampering early detection and treatment.

\section{MEAT CONSUMPTION AND CANCER RISK}

In the Netherlands, 1 out of 2.3 men will develop cancer at some point in his life, and the corresponding chance for women is 1 out of 2.6 [9]. The burden of cancer continues to increase in the future as a result of demographic changes and increasing adoption of cancer-causing lifestyle choices. This increase in number of cancer patients is expected to exert a substantial stressor on our health care system. It is thus important that we intensify our efforts for cancer prevention in order to decrease cancer incidence in the future.

The outcomes of the studies presented in chapter 4-9 are of high importance from an economic perspective since effective cancer prevention strategies will reduce the enormous health care costs associated with cancer treatment. From a societal perspective it is important to prevent cancer occurrence or improve cancer prognosis because this will greatly increase the quality of life of the individual.

Diet, including meat intake, is long hypothesized to be a modifiable risk factor for several cancers providing opportunities for cancer prevention. In the Netherlands, low(er) meat consumption is gaining popularity over the last years [10]. We set out to study if meat consumption was associated with cancer risk using a dietary pattern approach and a mechanistic approach.

\section{Dietary pattern approach}

Our descriptive analyses in Chapter 4 indicated that vegetarian and low meat consumers adhere to a lifestyle that is characterized by a more favorable distribution of dietary and lifestyle factors. This study provided a first unique and detailed insight into the characteristics and health status of an elderly population of vegetarians and low meat consumers in the Netherlands.

We reported that self-definition is not a reliable indicator for estimating the prevalence of vegetarianism in a population. Many nutrition examination surveys, however, use this as a classification tool to estimate and the number of individuals adhering to a vegetarian 
lifestyle. Since such data is used to direct and design health programs and services, it is important to use accurate classification tools.

Although we found little evidence that the abstinence of meat per se reduces the risk of the major cancers under study, it does become evident that the total vegetarian and low meat lifestyle may promote health and wellbeing. This is supported by studies showing lower diabetes, cardiovascular disease, and mortality rates among individuals adhering to such lifestyles [11-13]. If careful attention is being paid to preventing nutritional deficiencies in vegetarians (e.g. vitamin B12 - see above), public health policy may consider promoting the adaption of a balanced vegetarian lifestyle.

We also observed that a large percentage $(>10 \%)$ of the vegetarians and pescetarians in the NLCS-MIC cohort switched to this lifestyle after a cancer diagnosis. This suggests that cancer survivors may be highly motivated to make dietary changes towards a more plantbased diet after diagnosis with the intention to improve their health and well-being. While proper nutrition is important for cancer patients [14], it remains to be elucidated whether adopting a vegetarian lifestyle may influence the course of cancer prognosis and the risk of recurrence. Nutritional prognosis research is still in its infancy compared to etiologic research in cancer. With the aging of the population and rapid increase in the number of cancer patients and survivors, however, there is a great need for evidence based dietary recommendations for this specific target group. Some cohorts comprising a uniquely large number of cancer survivors have recently been initiated to investigate how diet and supplement use improve cancer survival and quality of life. This knowledge can eventually be used to develop protocols for nutritional care in cancer patients.

Vegetarians choose to adopt a meat-free diet for a variety of reasons. While this thesis examines the health effects of these diets, there are also a number of recent societal and economic effects associated with adopting a vegetarian diet making this a timely topic. Some of the environmental effects that are claimed to be associated with meat production include pollution through fossil fuel usage, and water and land consumption. In addition, the United Nations Sustainable Development Knowledge Platform recommends a substantial reduction of meat consumption and suggests that achieving such a sustainable meat production and consumption is essential in the transformation towards a more sustainable society.

\section{Mechanistic approach}

Our studies into specific mechanisms of meat-related carcinogenesis provided important biological insights that can potentially be used to develop (commercial) dietary prevention strategies. Chapter 8 suggests that heme iron may increase the risk of colorectal cancer primarily through DNA-alkylating processes as a result heme-catalyzed endogenous formation of $\mathrm{N}$-nitroso compounds (NOC). With this knowledge it may be possible to change or intervene on the production processes that lead to NOC formation. Several promising examples include adding calcium carbonate or vitamin $\mathrm{C}$ or $\mathrm{E}$ to the diet. If these interventions are fine-tuned and proven to be effective in an experimental setting, people may be able reduce their cancer risk associated with red meat consumption in the future.

In Chapter 8 we studied gene-environment $(\mathrm{G} \times \mathrm{E})$ interactions in relation to meat carcinogens and the risk of colorectal neoplasia. Such GxE studies may help to identify individuals at highest risk of cancer based on both their meat consumption patterns and genetic risk profiles. This knowledge may eventually be used to tailor interventions aimed 
at cancer prevention on genotype status. Before this can be applied on a large scale some (large) hurdles should be taken, including ethical, scientific and commercial issues. Some promising initiatives, however, are currently exploring the possibilities of commercially offering a personalized dietary prevention service based on genotyping data. G×E studies as described in this thesis form the scientific basis of such enterprises.

To conclude, this addendum illustrated how the research described in this thesis could results into social and economic knowledge translation. It is, however, important to point out that proper follow-up of our findings and recommendations are necessary before such knowledge transfer can be successful.

\section{REFERENCES}

1. Flegal KM, Carroll MD, Ogden CL, Curtin LR. Prevalence and trends in obesity among US adults, 19992008. Jama; 303(3): 235-41.

2. Adams KF, Schatzkin A, Harris TB, et al. Overweight, obesity, and mortality in a large prospective cohort of persons 50 to 71 years old. The New England journal of medicine 2006; 355(8): 763-78.

3. Renehan AG, Tyson M, Egger M, Heller RF, Zwahlen M. Body-mass index and incidence of cancer: a systematic review and meta-analysis of prospective observational studies. Lancet 2008; 371(9612): 569-78.

4. World Cancer Research Fund/American Institute for Cancer Research. Food, Nutrition, Physical Activity, and the Prevention of Cancer: a Global Perspective. Washington DC: AICR, 2007.

5. Guo SS, Zeller C, Chumlea WC, Siervogel RM. Aging, body composition, and lifestyle: the Fels Longitudinal Study. The American journal of clinical nutrition 1999; 70(3): 405-11.

6. Sheehan TJ, DuBrava S, DeChello LM, Fang Z. Rates of weight change for black and white Americans over a twenty year period. Int J Obes Relat Metab Disord 2003; 27(4): 498-504.

7. Stabler SP. Vitamin B12 deficiency. The New England journal of medicine 2013; 368(21): 2041-2.

8. Gezondheidsraad. Naar een optimaal gebruik van foliumzuur. Den Haag 2008; publicatienr. 2008/02. ISBN 978-90-5549-695-2

9. www.iknl.nl.

10. Dagevos H, Voordouw J, Hoeven Lv, Weele cvd, Bakker Ed. Vlees vooral(snog) vanzelfsprekend: consumenten over vlees eten en vleesminderen. LEU-rapport 2012-029. Den Haag, 2012.

11. Tonstad S, Stewart K, Oda K, Batech M, Herring RP, Fraser GE. Vegetarian diets and incidence of diabetes in the Adventist Health Study-2. Nutr Metab Cardiovasc Dis 2011.

12. Key TJ, Appleby PN, Spencer EA, Travis RC, Roddam AW, Allen NE. Mortality in British vegetarians: results from the European Prospective Investigation into Cancer and Nutrition (EPIC-Oxford). The American journal of clinical nutrition 2009; 89(5): 1613S-9S.

13. Key TJ, Fraser GE, Thorogood M, et al. Mortality in vegetarians and nonvegetarians: detailed findings from a collaborative analysis of 5 prospective studies. The American journal of clinical nutrition 1999; 70(3 Suppl): 516S-24S.

14. Pekmezi DW, Demark-Wahnefried W. Updated evidence in support of diet and exercise interventions in cancer survivors. Acta oncologica (Stockholm, Sweden) 2011; 50(2): 167-78. 

E

Etcetera 


\section{DANKWOORD}

Promoveren doe je niet alleen! De jaren van mijn promotieonderzoek heb ik ervaren als een bijzonder boeiende, uitdagende en meestal erg leuke en leerzame periode. Nu deze periode bijna is afgesloten kijk ik met trots terug op de dingen die ik doe en die ik de afgelopen jaren heb bereikt. Een groot woord van dank aan alle mensen die mij daarbij geholpen hebben en met wie ik mijn avonturen heb kunnen delen en beleven.

(co)Promotoren: Allereerst natuurlijk Leo. Het is alweer zeven jaar geleden dat jij mij als stagiaire onder je hoede nam en de tijd is voorbij gevlogen. Wat heb ik veel van je geleerd de afgelopen jaren. Ik heb je persoonlijke betrokkenheid bij mijn promotieonderzoek buitengewoon gewaardeerd en ben je zeer dankbaar dat jij mij altijd hebt gestimuleerd mijn eigen weg te volgen. Zonder jouw hulp en enthousiasme waren mijn buitenlandse stages nooit zo eenvoudig tot stand gekomen. Matty, ik had de luxe om jou als tweede 'dagelijkse' begeleider in mijn promotieteam te hebben. Dankzij jouw open en prettige manier van werken had ik altijd het gevoel dat ik voor alles bij je aan kon kloppen. Ik ken weinig mensen met zo'n enthousiasmerend vermogen en drive voor het onderzoek bedankt voor al je hulp, Matty! Piet, ik wil je bedanken dat je mij de mogelijkheid hebt gegeven binnen de NLCS te promoveren. Je overzag het geheel en hield de hoofdlijnen van het project altijd goed in te gaten. Ook op onderwijsgebied heb ik altijd prettig met je samengewerkt.

Co-auteurs: Aan de artikelen in dit proefschrift hebben veel co-auteurs een bijdrage geleverd. Ik wil iedereen dan ook hartelijk danken voor het kritisch becommentariëren van mijn stukken. Sandra, een speciaal woord van dank voor jou: jouw enthousiasme over en onuitputtelijke kennis van de voedingsepidemiologie is ongeëvenaard! Pieter, als 'nietNLCS-er' heb je een waardevolle bijdrage geleverd aan het vegetariër-project. Dank daarvoor!

Collega's Epidemiologie: Ik heb een fijne tijd gehad op de vakgroep Epidemiologie. Allemaal bedankt voor de gezellige sfeer en betrokkenheid op de afdeling. In het bijzonder wil ik Yvonne, Mariëlle, Petra, Jolanda, Sacha, Conny, Jos en Harry bedanken voor de geweldige ondersteuning. Jullie maken het leven van een promovenda een stuk makkelijker! Henny Brants wil ik bedanken voor de waardevolle input bij de classificatie van de vegetariërs.

De club hippe aio's en oud-aio's van Deb1 en Uns40: Jullie laten zien dat epidemiologen echt geen geitenwollen sokken meer dragen! Door de jaren heen zijn we een hecht groepje geworden. Mede door jullie kwam ik elke dag graag naar het werk. Ik denk met veel plezier terug aan de vele etentjes, borrels en natuurlijk de PhD tour. Ik vind het dan ook fijn dat ik met veel van jullie nog steeds contact heb. Ivette en Nadine, onze hardlooprondjes, minivakantie naar Dublin en daarna de vele speeldates met Niels vormden de ideale gelegenheid om gezellig over van-alles-en-nog-wat te kletsen. Ik ben blij dat ik jullie tijdens onze gezamelijke epi-jaren heb leren kennen!

Milan en Nadine: Zonder jullie als kamergenoten zou mijn promotieonderzoek een stuk minder gezellig zijn geweest. Wat hebben we de afgelopen vier jaar veel meegemaakt en 
gelachen met zijn drieën. Het laatste jaar hebben we veel gesproken over onze toekomstplannen en ik ben blij dat we nu alle drie onze eigen dromen najagen. Ik mis jullie nu al!

Martien van Dongen: Bedankt voor de meest briljante quote die ik tijdens mijn promotieonderzoek heb gehoord. Ik vind hem dan ook heel toepasselijk in deze epiloog van mijn proefschrift: 'Epi liegt niet, epi vertelt de waarheid'. Jij en Christel van Gool zijn het levende bewijs dat Brabantse krullenbollen prima epidemiologen zijn. Ik heb dan ook de stiekeme hoop dat de 'golden triangle' de komende jaren misschien ooit zal worden uitgebreid tot 'diamanten vierkant' - de tijd zal het leren....

Colleagues in Oxford: Francesca Crowe and Tim Key, your enthusiasm and passion for research have strengthened my conviction to pursue a career in science. I have truly enjoyed my time at the Cancer Epidemiology Unit during my Masters, and have great memories of my time in Oxford. It was wonderful to see everyone again during the PhD tour.

Colleagues @ NCl: Rashmi Sinha, Amanda Cross, Liz Ruder, and Sonja Berndt. I learned so much during my five month work visit at the US National Cancer Institute. It was a great experience being part of such a coherent team. A big thank you to everyone from the Nutritional Epidemiology Branch for making my time at $\mathrm{NCl}$ so much fun!

Lieke: Wat hebben we een hoop meegemaakt de afgelopen 20 jaar: van de atletiekbaan en de middelbare school in Helmond, via onze studententijd in Maastricht en ons avontuur in Finland naar het prachtige Deb1. Ik vind het dan ook heel bijzonder dat we nu één week na elkaar promoveren en jij op deze dag achter mij wilt staan. Kiitos!

Familie: Pap en mam, hoe ver ik ook van jullie vandaan woon, de Kapelweg blijft altijd mijn thuis! Heel erg bedankt voor alle kansen die jullie mij hebben gegeven en jullie eindeloze vertrouwen, liefde, en geduld. Dit proefschrift is voor jullie! Guus, jij hebt de afgelopen jaren waarschijnlijk het vaakst van iedereen gevraagd wat ik nu precies voor onderzoek doe. Hopelijk ben je als je dit boekje leest dan ook net zo trots op mij als ik op jou! Mijn lieve schoonfamilie, Frank en Rita, Karin en Robert, en Ilze en Tom (en Teun!): het voelt goed om te weten dat jullie er altijd voor ons zijn.

Tim en Niels: Jullie zijn mijn grootste geluk. Wat hebben wij het toch goed met zijn drietjes. Ik ben ontzettend trots op ons en verheug me op wat de toekomst ons zal brengen.

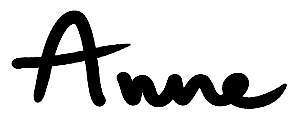

Hamilton, oktober 2014 


\section{ABOUT THE AUTHOR}

Anne Gilsing was born on February $16^{\text {th }}, 1986$ in Helmond, the Netherlands. After completing secondary school (Atheneum) at the Carolus Borromeus College in Helmond in 2004, she studied Health Sciences at Maastricht University, in Maastricht, the Netherlands. Three years later, including four months abroad at the University of Jyväskylä (Finland) and completing the extracurricular honours program, Anne obtained her BSc. Degree (cum laude). In 2007, Anne enrolled in the two year Health Sciences Research Master's program at Maastricht University (specialization clinical epidemiology), and obtained her MPhil degree (cum laude) in 2009. For her thesis, Anne conducted a six month internship at the Cancer Epidemiology Unit at the University of Oxford (United Kingdom). After graduating, Anne was awarded a one year Kootstra Talent Fellowship from the Board of Directors from Maastricht University for talented young researchers during which she worked as a junior researcher at the Department of Epidemiology. During her Masters and fellowship year, Anne wrote a project proposal in collaboration with her supervisors focusing on the association between vegetarianism, low meat consumption and the risk of major cancers in the Netherlands Cohort Study. After this proposal was funded by the World Cancer Research Fund (WCRF), Anne started her PhD project in August 2010. In the second year of Anne's PhD project she completed a five month work visit to the US National Cancer Institute where she performed novel data reduction techniques to identify genetic variants involved in gene $x$ environment interactions. In 2011, Anne initiated and co-organized an eight-day scientific study tour for PhD students from the Department of Epidemiology to the United Kingdom. Since the summer of 2014, Anne works as a postdoctoral fellow at the Department of Clinical Epidemiology and Biostatistics at McMaster University, Hamilton, Canada where she continues her work in the field of nutrition, cancer and healthy aging. 


\section{LIST OF PUBLICATIONS}

\section{Submitted:}

1. Gilsing AMJ, Weijenberg MP, Goldbohm RA, Dagnelie PC, van den Brandt PA, Schouten $\sqcup$. Vegetarianism, low meat consumption and the risk of lung, prostate and female breast cancer in a population based cohort study.

\section{Accepted for publication:}

2. Gilsing AMJ, Schouten LJ, Goldbohm RA, Dagnelie PC, van den Brandt PA, Weijenberg MP. Colorectal cancer risk in vegetarians and low meat consumers: a prospective investigation.

\section{Appeared:}

3. Ruder EH, Berndt SI, Gilsing AMJ, Graubard BI, Ferrucci LM, Burdett L, Weissfeld JL, Cross AJ, Sinha R. Dietary iron, iron homeostatic gene polymorphisms and the risk of advanced colorectal adenoma and cancer. Carcinogenesis. 2014 Feb (epub ahead of print)

4. Gilsing AMJ, Weijenberg MP, Goldbohm RA, Dagnelie PC, van den Brandt PA, Schouten L. The Netherlands Cohort Study - Meat Investigation Cohort; A population-based cohort oversampled with vegetarians, pescetarians and low meat consumers. Nutrition Journal. 2013; 29;12:156.

5. Gilsing AMJ, Franssen F, de Kok T, de Goeij A, de Bruine A, van Engeland M, van den Brandt PA, Weijenberg MP. Dietary heme is associated with colorectal cancer with $G>A$ mutations in KRAS and APC genes. Carcinogenesis. 2013; 34(12):2757-66.

6. Gilsing AMJ, Berndt SI, Ruder EH, Graubard BI, Ferrucci LM, Burdett L, Weissfeld JL, Cross AJ, Sinha R. Meat-related mutagen exposure, xenobiotic metabolizing gene polymorphisms and the risk of advanced colorectal adenoma and cancer. Carcinogenesis. 2012 July;33(7):1332-1339.

7. Gilsing AMJ, Weijenberg MP, Hughes LA, Ambergen T, Dagnelie PC, Goldbohm RA, Brandt PA, Schouten L. Longitudinal Changes in BMI in Older Adults Are Associated with Meat Consumption Differentially, by Type of Meat Consumed. J Nutr. 2012 Feb;142(2):340-9.

8. Gilsing AMJ, Weijenberg MP, Goldbohm RA, van den Brandt PA, Schouten LJ. Consumption of dietary fat and meat and risk of ovarian cancer in the Netherlands Cohort Study. Am J Clin Nutr. 2011 Jan;93(1):118-26.

9. Gilsing AMJ, Crowe FL, Lloyd-Wright Z, Sanders TA, Appleby PN, Allen NE, Key TJ. Serum concentrations of vitamin B12 and folate in British male omnivores, vegetarians and vegans: results from a cross-sectional analysis of the EPIC-Oxford cohort study. Eur J Clin Nutr. 2010 Sep;64(9):933-9. 
